\title{
Site-Selective Thiolation of (Multi)halogenated Heteroarenes
}

Frederik Sandfort, Tobias Knecht, Tobias Pinkert, Constantin G. Daniliuc, and Frank Glorius*

Supporting Information

Organisch-Chemisches Institut, Westfälische Wilhelms-Universität Münster, Corrensstraße 40, 48149 Münster, Germany

*glorius@uni-muenster.de 


\section{Content}

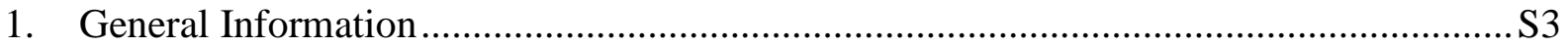

2. Investigation of the Thiolation of Halogenated Heteroarenes ..........................................S6

2.1. Synthesis of Catalysts and Starting Materials .........................................................S6

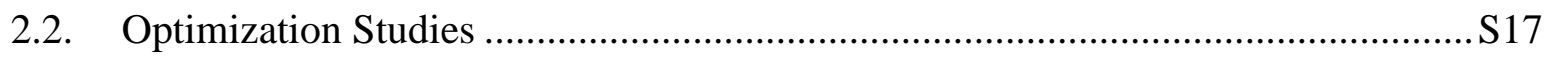

2.3. Sensitivity Assessment .......................................................................................

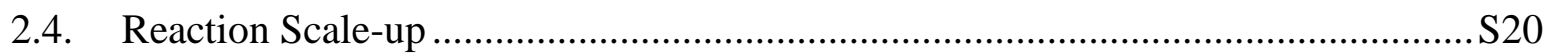

2.5. Substrate Scope and Characterization Data...................................................... S21

2.6. Methylthiolation of Halogenated Heteroarenes................................................... S40

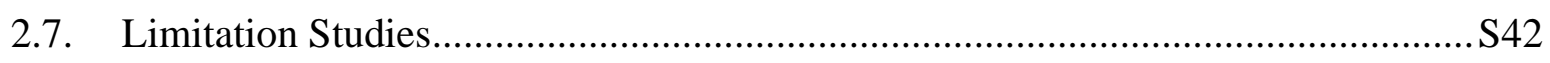

2.8. Additive-Based Robustness Screen .................................................................... 43

2.9. Competition Experiment of Chlorinated and Brominated Substrates ......................S45

3. Comparison to Established C-S Coupling Protocols......................................................S46

3.1. Palladium-Catalyzed Cross-Coupling …………………...................................... 44

3.2. Nickel-Photoredox Dual-Catalytic Coupling …………………………….......... 44

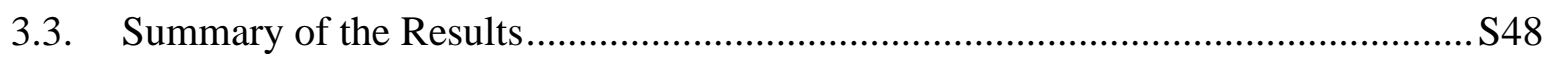

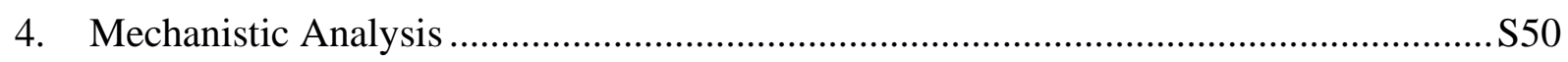

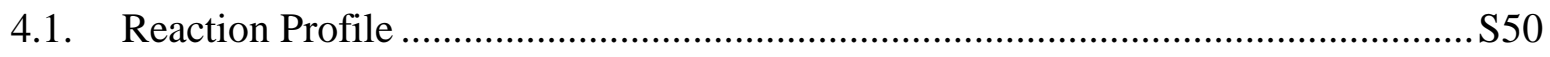

4.2. Stern-Volmer Quenching Studies ..................................................................... S51

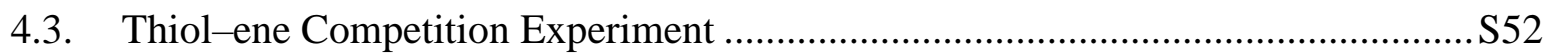

4.4. Investigation of Traditional Radical Initiators........................................................ S54

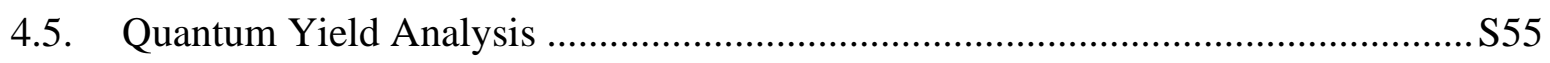

4.6. Computational Investigation of the Reaction Mechanism ......................................S57

4.7. Computational Determination of Redox Potentials ...................................................S64

4.8. Mechanistic Considerations Regarding the Methylthiolation ................................S71

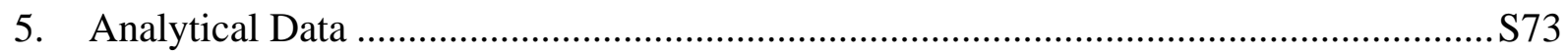

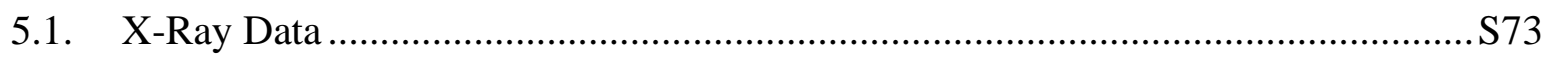

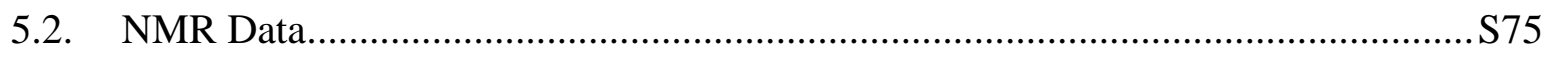

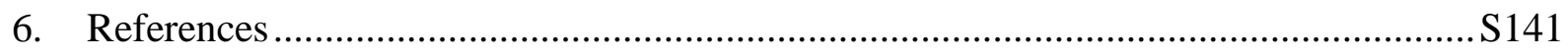




\section{General Information}

Unless otherwise noted, all reactions were carried out under an atmosphere of argon in ovendried glassware. Anhydrous solvents were either purchased and stored over molecular sieves under argon, or purified by distillation over standard drying agents. Reaction temperatures are reported as the temperature of the medium surrounding the vessel. Commercially available chemicals were used as received if not stated otherwise.

Photochemical reactions were performed in a Hepatochem EvoluChem ${ }^{\mathrm{TM}}$ PhotoRedOx Box Duo device (see Figure S1) and irradiated with two EvoluChem ${ }^{\mathrm{TM}}$ P303-30-1 LEDs (30 W, $\lambda_{\max }$ $=450 \mathrm{~nm}$, see Figure S2). The reaction temperature was measured to be between $30{ }^{\circ} \mathrm{C}$ and $35^{\circ} \mathrm{C}$ using this setup. The quantum yield was determined using a single LED ( $3 \mathrm{~W} \lambda_{\max }=$ $420 \mathrm{~nm}$, see Figure S3).
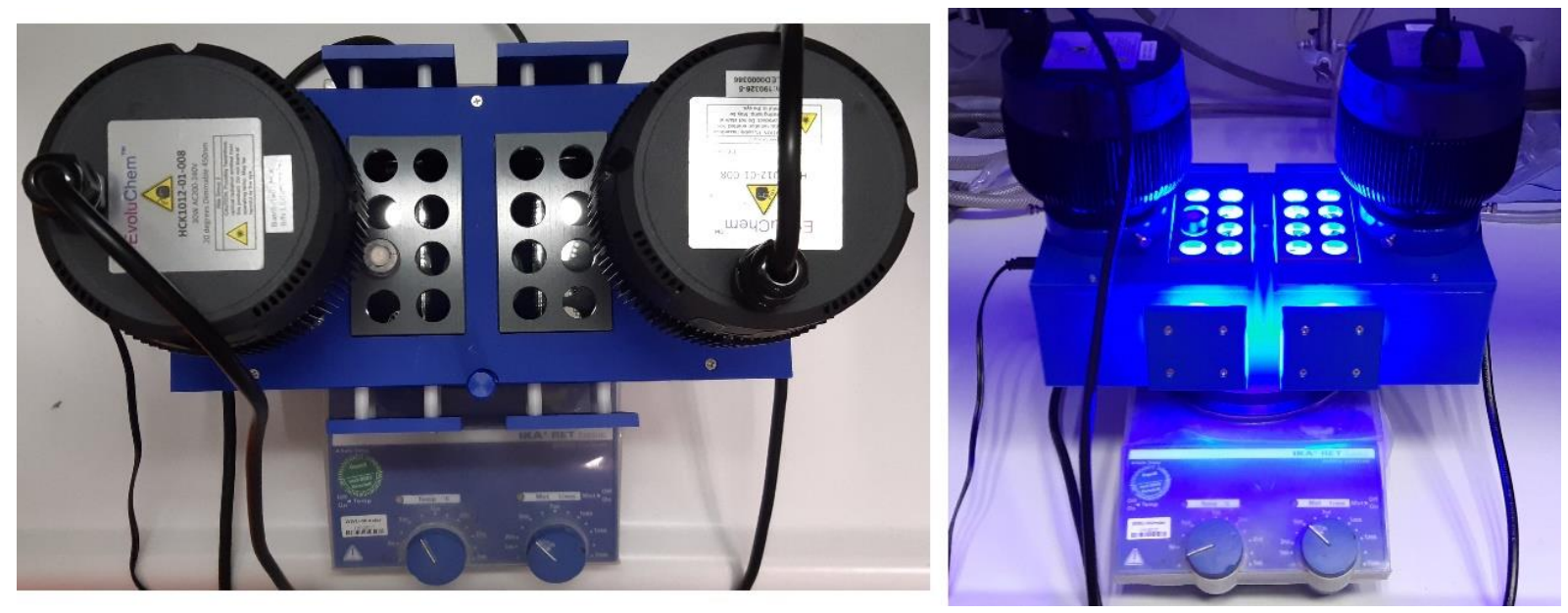

Figure S1. Hepatochem EvoluChem ${ }^{\mathrm{TM}}$ PhotoRedOx Box Duo device.

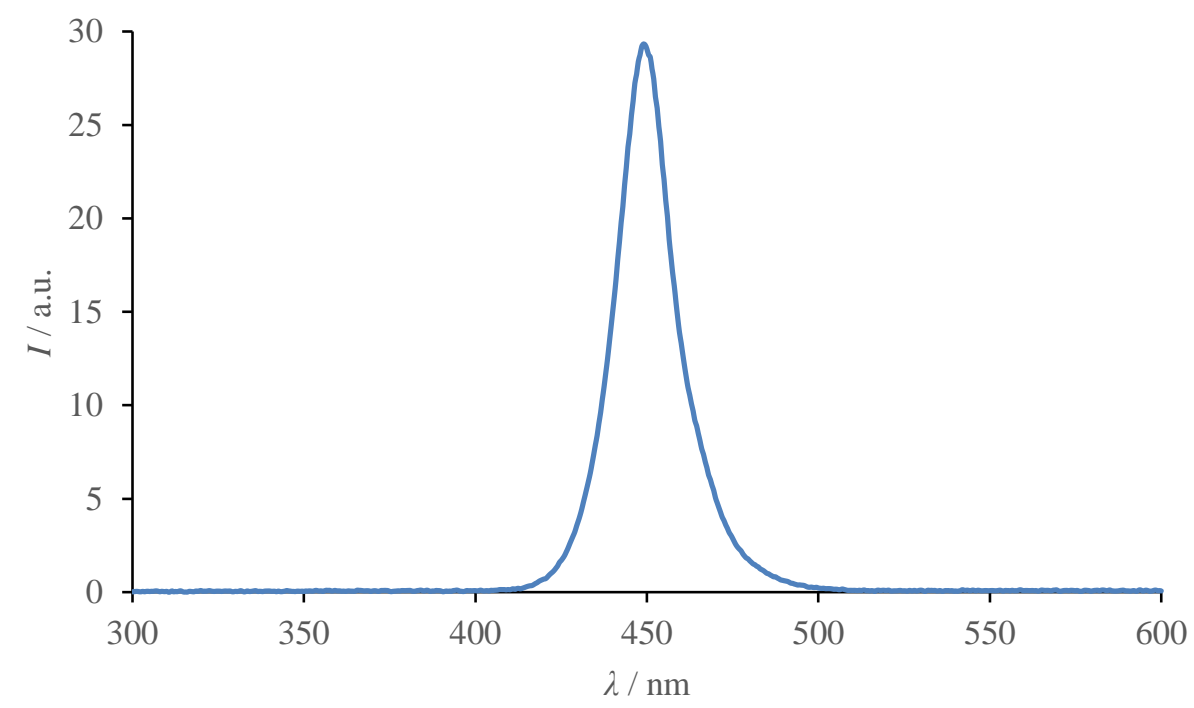

Figure S2. Emission spectra of the EvoluChem ${ }^{\mathrm{TM}}$ P303-30-1 LEDs $\left(30 \mathrm{~W}, \lambda_{\max }=450 \mathrm{~nm}\right)$ used for reaction irradiation. 


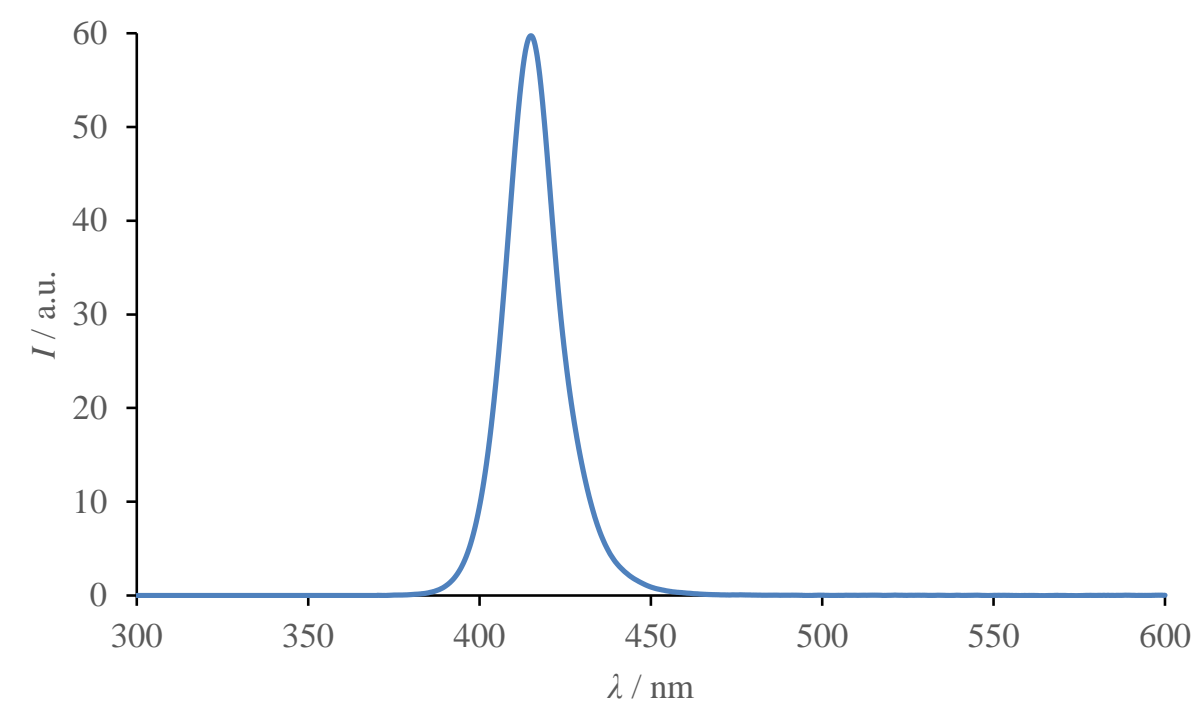

Figure S3. Emission spectra of the single blue LED $\left(3 \mathrm{~W}, \lambda_{\max }=420 \mathrm{~nm}\right)$ used for quantum yield determination.

Analytical thin layer chromatography (TLC) was performed on silica gel 60 F254 aluminum plates (Merck). TLC plates were visualized by exposure to short wave ultraviolet light (254 nm, $366 \mathrm{~nm}$ ) and were dipped into a solution of $\mathrm{KMnO}_{4}$. Flash chromatography was performed on Acros Organics silica gel (35-70 mesh) under a positive pressure of argon, eluting with the specified solvent system.

NMR-spectra were recorded on a Bruker Avance II 300, Bruker Avance II 400, Agilent DD2 500 or on an Agilent DD2 600 spectrometer. Chemicals shifts $(\delta)$ are quoted in ppm downfield of tetramethylsilane. The residual solvent signals were used as references for ${ }^{1} \mathrm{H}$ and ${ }^{13} \mathrm{C} \mathrm{NMR}$ spectra $\left(\mathrm{CDCl}_{3}: \delta \mathrm{H}=7.26 \mathrm{ppm}, \delta \mathrm{C}=77.16 \mathrm{ppm} ; \mathrm{CD}_{3} \mathrm{OD}: \delta \mathrm{H}=3.31 \mathrm{ppm}, \delta \mathrm{C}=49.00 \mathrm{ppm}\right.$; DMSO-d 6 : $\delta \mathrm{H}=2.50 \mathrm{ppm}, \delta \mathrm{C}=39.52 \mathrm{ppm}) .{ }^{19} \mathrm{~F}$ NMR spectra are not calibrated by an internal reference. The multiplicity of all signals was described with standard abbreviations. Coupling constants $(J)$ are quoted in $\mathrm{Hz}$.

GC-MS spectra were recorded on an Agilent Technologies 7890A GC-system with an Agilent 5975C VL MSD or an Agilent 5975 inert Mass Selective Detector (EI) and a HP-5MS column $(0.25 \mathrm{~mm} \times 30 \mathrm{~m}$, film: $0.25 \mu \mathrm{m})$. The major signals are quoted in $\mathrm{m} / \mathrm{z}$ with the relative intensity in parentheses. The method indicated as ' $50 \_40$ ' starts with the injection temperature $T_{0}$ $\left(50{ }^{\circ} \mathrm{C}\right.$ ); after holding this temperature for $3 \mathrm{~min}$, the column is heated by $40{ }^{\circ} \mathrm{C} / \mathrm{min}$ to temperature $T_{1}\left(290{ }^{\circ} \mathrm{C}\right.$ or $\left.320^{\circ} \mathrm{C}\right)$ and this temperature is held for an additional time $t$. GCFID analysis was undertaken on an Agilent Technologies 6890A equipped with an HP-5 quartz column $(0.32 \mathrm{~mm}$ x $30 \mathrm{~m}$, film: $0.25 \mu \mathrm{m})$ using flame ionization detection. Method: Initial temperature $50{ }^{\circ} \mathrm{C}$, hold $3 \mathrm{~min}$, increment $40{ }^{\circ} \mathrm{C} / \mathrm{min}$, final temperature $280{ }^{\circ} \mathrm{C}$, hold $3 \mathrm{~min}$. High resolution mass spectra (HRMS) were obtained by the MS service of the OrganischChemisches Institut, Westfälische Wilhelms-Universität Münster, using electrospray ionisation (ESI) on a Bruker Daltonics MicroTof spectrometer.

Stern-Volmer luminescence quenching analysis was conducted using a Jasco FP-8300 spectrofluorometer. The following parameters were employed: Excitation bandwidth $=5 \mathrm{~nm}$, data interval $=0.2 \mathrm{~nm}$, scan speed $=500 \mathrm{~nm} / \mathrm{min}$, response time $=0.2 \mathrm{sec}$. 
Data sets for compound 3ae were collected with a Bruker D8 Venture CMOS diffractometer. For compound 3g data sets were collected with a Bruker APEX II CCD diffractometer. Programs used: data collection: APEX3 V2016.1-0; ${ }^{31}$ cell refinement: SAINT V8.37A; ${ }^{31}$ data reduction: SAINT V8.37A $;^{31}$ absorption correction, SADABS V2014/7; ${ }^{31}$ structure solution SHELXT-2015; ${ }^{32}$ structure refinement SHELXL-2015 $5^{33}$ and graphics, $X P .{ }^{34} R$-values are given for observed reflections, and $w \mathrm{R}^{2}$ values are given for all reflections. 


\section{Investigation of the Thiolation of Halogenated Heteroarenes}

\subsection{Synthesis of Catalysts and Starting Materials}

The photocatalysts $\left[\operatorname{Ir}\left(\mathrm{dFCF}_{3} \text { ppy }\right)_{2}(\mathrm{dtbbpy})\right] \mathrm{PF}_{6},{ }^{35}\left[\operatorname{Ir}(\mathrm{ppy})_{2}(\mathrm{dtbbpy})\right] \mathrm{PF}_{6},{ }^{36} \mathrm{fac}$ - $\operatorname{Ir}(\mathrm{ppy})_{3},{ }^{37}$ $\left[\mathrm{Ru}(\mathrm{bpy})_{3}\right]\left(\mathrm{PF}_{6}\right)_{2},{ }^{38} 4 \mathrm{CzIPN}^{39}$ were prepared following literature procedures.

Synthesis of 1-benzyl-2-bromo-4,5-dichloro-1H-imidazole (1h)

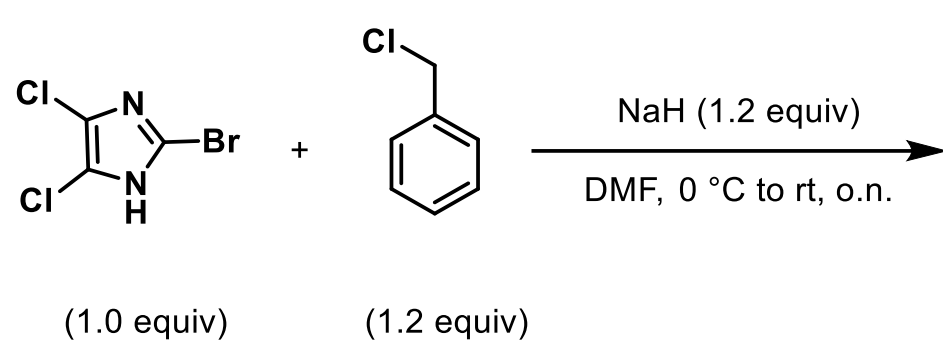

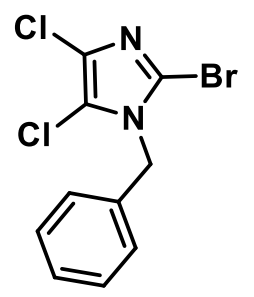

$1 \mathrm{~h}$

According to a modified procedure by Fagnou et al. ${ }^{40}$ 2-bromo-4,5-dichloro-imidazole (2.14, $10 \mathrm{mmol}, 1.0$ equiv) was dissolved in dry DMF $(20 \mathrm{~mL}, 0.5 \mathrm{M})$ and cooled to $0{ }^{\circ} \mathrm{C}$. $\mathrm{NaH}(60 \%$ in mineral oil, $480 \mathrm{mg}, 12 \mathrm{mmol}, 1.2$ equiv) was added in small portions and the resulting suspension was stirred for $1 \mathrm{~h}$ at $0{ }^{\circ} \mathrm{C}$. Afterwards, benzyl chloride $(1.38 \mathrm{~mL}, 12 \mathrm{mmol}, 1.2$ equiv.) was added and the solution was allowed to come to room temperature overnight. The reaction mixture was carefully quenched with $\mathrm{H}_{2} \mathrm{O}(20 \mathrm{~mL})$ and diluted with EtOAc $(40 \mathrm{~mL})$. The organic layer was extracted with $\mathrm{H}_{2} \mathrm{O}(2 \times 20 \mathrm{~mL})$, dried over $\mathrm{MgSO}_{4}$ and concentrated under reduced pressure. The residue was recrystallized from DCM/pentane to afford the desired product as an off-white solid (561 mg, $1.9 \mathrm{mmol}, 19 \%)$.

${ }^{1}$ H NMR (400 MHz, Chloroform-d) $\delta 7.42-7.30$ (m, 3H), $7.21-7.14$ (m, 2H), 5.16 (s, 2H); ${ }^{13}$ C NMR (101 MHz, Chloroform-d) $\delta$ 134.1, 129.2, 128.6, 127.1, 126.6, 116.7, 115.3, 50.2; HR-MS (ESI) $\mathrm{m} / \mathrm{z}$ calculated for $\left[\mathrm{C}_{10} \mathrm{H}_{7} \mathrm{~N}_{2} \mathrm{BrCl}_{2} \mathrm{Na}\right]^{+}\left([\mathrm{M}+\mathrm{Na}]^{+}\right)$: 328.9040 , measured: 328.9040 .

Synthesis of methyl (R)-2-((tert-butoxycarbonyl)amino)-3-(5-chloro-1-methyl-1H-imidazol-4yl)propanoate (1i)
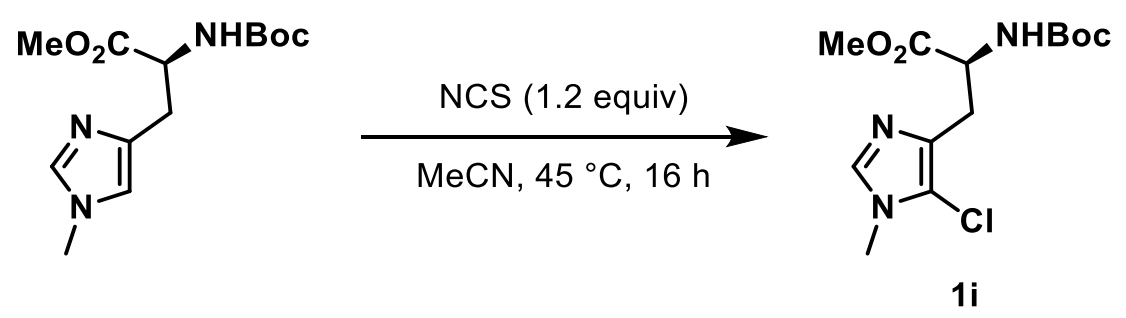

In an oven-dried Schlenk flask containing a magnetic stirring bar, methyl $N^{\alpha}$-(tertbutoxycarbonyl)- $N^{\tau}$-methyl- $L$-histidinate ${ }^{43}$ (425 mg, $1.5 \mathrm{mmol}, 1.0$ equiv) was dissolved in MeCN (15 mL) under argon. $N$-Chlorosuccinimide (NCS, $240 \mathrm{mg}, 1.8 \mathrm{mmol}, 1.2$ equiv) was added portion-wise and the reaction was stirred at $45^{\circ} \mathrm{C}$ for $16 \mathrm{~h}$. The mixture was allowed to cool to room temperature and the solvent was removed under reduced pressure. The crude 
product was purified by column chromatography (EA $-\mathrm{EA}: \mathrm{MeOH}=50: 1)$ to afford the title compound as a yellowish solid (287 $\mathrm{mg}, 60 \%)$.

${ }^{1}$ H NMR (400 MHz, Chloroform-d) $\delta 7.38(\mathrm{~s}, 1 \mathrm{H}), 5.85(\mathrm{~d}, J=8.3 \mathrm{~Hz}, 1 \mathrm{H}), 4.55$ (dt, $J=8.3$, $4.9 \mathrm{~Hz}, 1 \mathrm{H}), 3.71(\mathrm{~s}, 3 \mathrm{H}), 3.55(\mathrm{~s}, 3 \mathrm{H}), 3.18-2.91(\mathrm{~m}, 2 \mathrm{H}), 1.43(\mathrm{~s}, 9 \mathrm{H}) ;{ }^{13} \mathbf{C}$ NMR $(101 \mathrm{MHz}$, Chloroform-d) $\delta 172.5,155.6,136.3,132.7,116.0,79.7,53.1,52.4,31.8,28.8,28.5$; $\mathbf{R}_{\mathbf{f}}$ (EtOAc): 0.17; HR-MS (ESI) $\mathrm{m} / \mathrm{z}$ calculated for $\left[\mathrm{C}_{13} \mathrm{H}_{20} \mathrm{~N}_{3} \mathrm{O}_{4} \mathrm{ClH}\right]^{+}\left([\mathrm{M}+\mathrm{H}]^{+}\right): 318.1215$, measured: 318.1219 .

Synthesis of methyl (S)-2-((tert-butoxycarbonyl)amino)-3-(2,5-dichloro-1-methyl-1Himidazol-4-yl)propanoate $(\mathbf{1 j})$
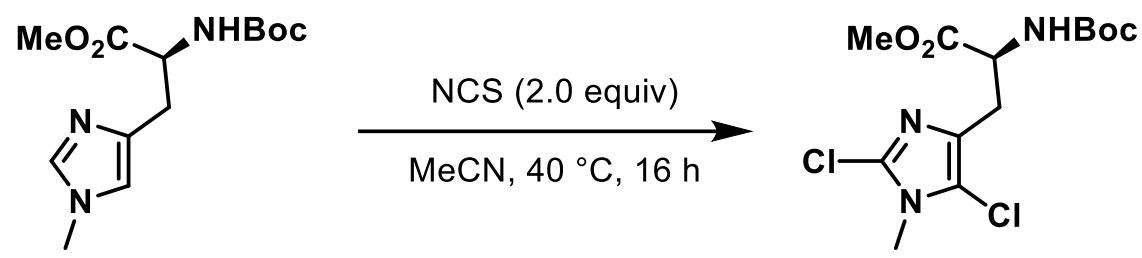

1j

In an oven-dried Schlenk flask containing a magnetic stirring bar, methyl $N^{\alpha}$-(tertbutoxycarbonyl)- $N^{\tau}$-methyl- $L$-histidinate ${ }^{43}(283 \mathrm{mg}, 1.0 \mathrm{mmol}, 1.0$ equiv) was dissolved in MeCN (10 mL) under argon. $N$-Chlorosuccinimide (NCS, $267 \mathrm{mg}, 2.0 \mathrm{mmol}, 2.0$ equiv) was added portion-wise and the reaction was stirred at $40{ }^{\circ} \mathrm{C}$ for $16 \mathrm{~h}$. The mixture was allowed to cool to room temperature and the solvent was removed under reduced pressure. The crude product was purified by column chromatography (pentane:EA $=2: 1-3: 2$ ) to afford the title compound as a yellowish solid (153 $\mathrm{mg}, 43 \%)$.

${ }^{1}$ H NMR (400 MHz, Chloroform-d) $\delta 5.58(\mathrm{~d}, J=8.4 \mathrm{~Hz}, 1 \mathrm{H}), 4.60-4.45$ (m, 1H), 3.73 (s, $3 \mathrm{H}), 3.51(\mathrm{~s}, 3 \mathrm{H}), 3.10-2.84(\mathrm{~m}, 2 \mathrm{H}), 1.43(\mathrm{~s}, 9 \mathrm{H}) ;{ }^{13} \mathrm{C}$ NMR (101 MHz, Chloroform-d) $\delta$ 172.2, 155.5, 132.1 130.8, 116.4, 79.9, 53.0, 52.5, 31.6, 29.1, 28.5; $\mathbf{R} \mathbf{f}($ pentane:EtOAc = 2:1): 0.27; HR-MS (ESI) $\mathrm{m} / \mathrm{z}$ calculated for $\left[\mathrm{C}_{13} \mathrm{H}_{19} \mathrm{Cl}_{2} \mathrm{~N}_{3} \mathrm{O}_{4} \mathrm{Na}\right]^{+}\left([\mathrm{M}+\mathrm{Na}]^{+}\right): 374.0645$, measured: 374.0650 .

Synthesis of 4,5-dichloro-1-(2-chlorobenzyl)-1H-imidazole (1k)
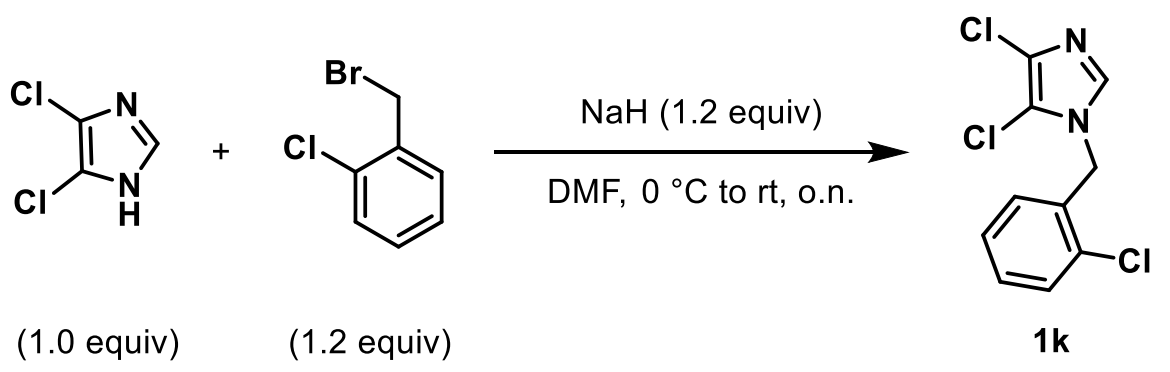

According to a modified procedure by Fagnou et al. ${ }^{40}$ 4,5-dichloroimidazole ( $685 \mathrm{mg}, 5.0$ mmol, 1.0 equiv) was dissolved in dry DMF $(10 \mathrm{~mL}, 0.5 \mathrm{M})$ and cooled to $0{ }^{\circ} \mathrm{C}$. $\mathrm{NaH}(60 \%$ in mineral oil, $240 \mathrm{mg}, 6.0 \mathrm{mmol}, 1.2$ equiv) was added in small portions and the resulting suspension was stirred for $1 \mathrm{~h}$ at $0{ }^{\circ} \mathrm{C}$. Afterwards, 1 -(bromomethyl)-2-chlorobenzene $(750 \mu \mathrm{L}$, 
$6.0 \mathrm{mmol}, 1.2$ equiv.) was added and the solution was allowed to come to room temperature overnight. The reaction mixture was carefully quenched with $\mathrm{H}_{2} \mathrm{O}(10 \mathrm{~mL})$ and diluted with EtOAc $(20 \mathrm{~mL})$. The organic layer was extracted with $\mathrm{H}_{2} \mathrm{O}(2 \times 10 \mathrm{~mL})$, dried over $\mathrm{MgSO}_{4}$ and concentrated under reduced pressure. The residue was purified by silica gel chromatography (pentane:EtOAc $=4: 1$ ) to afford the desired product as a colorless liquid that solidified upon standing (705 mg, $2.7 \mathrm{mmol}, 54 \%$ ).

${ }^{1}$ H NMR (400 MHz, Chloroform-d) $\delta 7.47-7.36$ (m, 2H), 7.27 (dtd, $J=18.4,7.5,1.6 \mathrm{~Hz}$, $2 \mathrm{H}), 6.95(\mathrm{dd}, J=7.5,1.9 \mathrm{~Hz}, 1 \mathrm{H}), 5.17(\mathrm{~s}, 2 \mathrm{H}) ;{ }^{13} \mathbf{C}$ NMR (101 MHz, Chloroform-d) $\delta$ $134.9,133.2,132.1,130.1,130.0,129.0,127.6,126.4,113.7,47.4 ; \mathbf{R}_{\mathbf{f}}($ pentante:EtOAc = 4:1): 0.41; HR-MS (ESI) $\mathrm{m} / \mathrm{z}$ calculated for $\left[\mathrm{C}_{10} \mathrm{H}_{7} \mathrm{Cl}_{3} \mathrm{~N}_{2} \mathrm{Na}\right]^{+}\left([\mathrm{M}+\mathrm{Na}]^{+}\right): 282.9567$, measured: 282.9584 .

Synthesis of 1-(4-bromobenzyl)-4,5-dichloro-1H-imidazole (11)

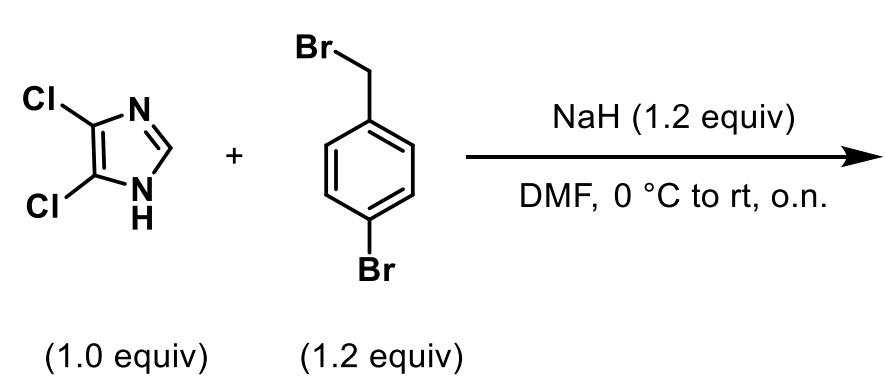

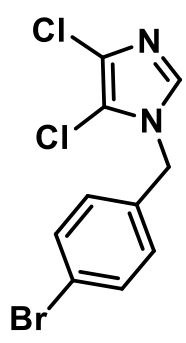

11

According to a modified procedure by Fagnou et al. ${ }^{40}$ 4,5-dichloroimidazole ( $685 \mathrm{mg}, 5.0$ mmol, 1.0 equiv) was dissolved in dry DMF $(10 \mathrm{~mL}, 0.5 \mathrm{M})$ and cooled to $0{ }^{\circ} \mathrm{C}$. $\mathrm{NaH}(60 \%$ in mineral oil, $240 \mathrm{mg}, 6.0 \mathrm{mmol}, 1.2$ equiv) was added in small portions and the resulting suspension was stirred for $1 \mathrm{~h}$ at $0{ }^{\circ} \mathrm{C}$. Afterwards, 1-bromo-4-(bromomethyl)benzene $(1.50 \mathrm{~g}$, $6.0 \mathrm{mmol}, 1.2$ equiv.) was added and the solution was allowed to come to room temperature overnight. The reaction mixture was carefully quenched with $\mathrm{H}_{2} \mathrm{O}(10 \mathrm{~mL})$ and diluted with EtOAc $(20 \mathrm{~mL})$. The organic layer was extracted with $\mathrm{H}_{2} \mathrm{O}(2 \times 10 \mathrm{~mL})$, dried over $\mathrm{MgSO}_{4}$ and concentrated under reduced pressure. The residue was recrystallized from DCM/pentane to afford the desired product as a white crystalline solid (825 mg, $2.7 \mathrm{mmol}, 54 \%)$.

${ }^{1} \mathbf{H}$ NMR (400 MHz, Chloroform-d) $\delta 7.58-7.46(\mathrm{~m}, 2 \mathrm{H}), 7.40(\mathrm{~s}, 1 \mathrm{H}), 7.09-7.00(\mathrm{~m}, 2 \mathrm{H})$, 5.03 (s, 2H); ${ }^{13} \mathbf{C}$ NMR (101 MHz, Chloroform-d) $\delta 134.5,133.5,132.4,129.1,126.7,122.9$, 113.7, 49.3; HR-MS (ESI) $\mathrm{m} / \mathrm{z}$ calculated for $\left[\mathrm{C}_{10} \mathrm{H}_{7} \mathrm{~N}_{2} \mathrm{BrCl}_{2} \mathrm{Na}\right]^{+}\left([\mathrm{M}+\mathrm{Na}]^{+}\right): 328.9040$, measured: 328.9048 . 
Synthesis of 6-chloro-N-(2-(4,5-dichloro-1H-imidazol-1-yl)ethyl)nicotinamide (1m)<smiles>Clc1nc[nH]c1Cl</smiles>

(1.1 equiv)<smiles>O=C1c2ccccc2C(=O)N1CCBr</smiles>

(1.0 equiv)

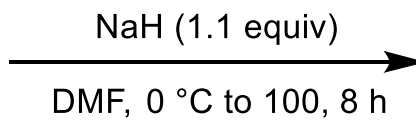<smiles>O=C1c2ccccc2C(=O)N1CCn1cnc(Cl)c1Cl</smiles>
$\mathrm{H}_{4} \mathrm{~N}_{2} \cdot \mathrm{H}_{2} \mathrm{O}$ ( 1.5 equiv) $\mathrm{EtOH}, \Delta, 4 \mathrm{~h}$<smiles>O=C(NCCn1cnc(Cl)c1Cl)c1ccc(Cl)nc1</smiles><smiles>CCCCCOc1cc(C(=O)Cl)ccc1Cl</smiles>

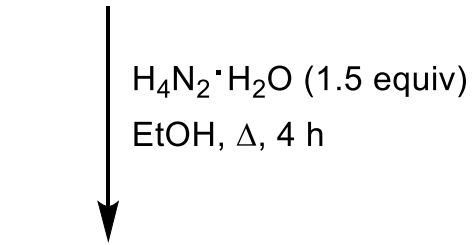<smiles>NCCn1cnc(Cl)c1Cl</smiles>

According to a modified procedure by Demuth et al. ${ }^{41}$ 4,5-dichloroimidazole (4.38 g, 32 mmol, 1.1 equiv) was dissolved in dry DMF $(40 \mathrm{~mL}, 0.8 \mathrm{M})$ and cooled to $0{ }^{\circ} \mathrm{C}$. $\mathrm{NaH}(60 \%$ in mineral oil, $1.28 \mathrm{mg}, 32 \mathrm{mmol}, 1.1$ equiv) was added in small portions and the resulting suspension was stirred for $1 \mathrm{~h}$ at $0{ }^{\circ} \mathrm{C}$. Afterwards, 2-(2-bromoethyl)isoindoline-1,3-dione (7.76 g, $30 \mathrm{mmol}$, 1.0 equiv) was added at $0{ }^{\circ} \mathrm{C}$ and the reaction mixture heated to $100{ }^{\circ} \mathrm{C}$ for $8 \mathrm{~h}$. The resulting suspension was diluted with EtOAc $(150 \mathrm{~mL})$ and washed with $\mathrm{H}_{2} \mathrm{O}(2 \times 150 \mathrm{~mL})$. The organic phase was dried over $\mathrm{MgSO}_{4}$ and the solvents were removed under reduced pressure.

According to a modified procedure by Wright and Press et al. ${ }^{42}$ the corresponding 2-(2-(4,5dichloro-1H-imidazol-1-yl)ethyl)isoindoline-1,3-dione (3.10 g, $10 \mathrm{mmol}, 1.0$ equiv) was dissolved in ethanol (20 mL, $0.5 \mathrm{M})$ followed by the dropwise addition of $\mathrm{H}_{4} \mathrm{~N}_{2} \cdot \mathrm{H} 2 \mathrm{O}(51 \%$ in water, $941 \mathrm{mg}, 15 \mathrm{mmol}, 1.5 \mathrm{equiv})$. The reaction mixture was subsequently heated to reflux for $4 \mathrm{~h}$. $\mathrm{HCl}$ (aq. $4 \mathrm{M}, 40 \mathrm{~mL}$ ) was added and the suspension was refluxed for another $2 \mathrm{~h}$. After cooling down to room temperature, ethanol was removed under reduced pressure, the aqueous phase was extracted with DCM $(2 \times 20 \mathrm{~mL})$ and the organic phase was disposed. The aqueous phase was basified with $\mathrm{NaOH}$ (aq. $4 \mathrm{M})$ and extracted with DCM (3 x $100 \mathrm{~mL})$. The combined organic layers were dried over $\mathrm{MgSO}_{4}$ and the solvent was removed under reduced pressure to yield the deprotected amine as a brown oil $(1.49 \mathrm{~g}, 8.3 \mathrm{mmol}, 83 \%)$.

6-Chloronicotinic acid (757 mg, $4.8 \mathrm{mmol}, 1.2$ equiv) in DCM (15 mL, $0.3 \mathrm{M})$ was treated with $\mathrm{DMF}$ (3 drops) followed by the dropwise addition of $(\mathrm{COCl})_{2}(455 \mu \mathrm{L}, 5.3 \mathrm{mmol} 1.3$ equiv) at 
$0{ }^{\circ} \mathrm{C}$. The reaction mixture was allowed to come to room temperature and stirred for $2 \mathrm{~h}$ and the volatiles were removed under reduced pressure. The corresponding nicotinoyl chloride was added to a solution of 2-(4,5-dichloro-1H-imidazol-1-yl)ethan-1-amine (720 mg, $4.0 \mathrm{mmol}, 1.0$ equiv) and $\mathrm{NEt}_{3}\left(1.1 \mathrm{~mL}, 8.0 \mathrm{mmol}, 2.0\right.$ equiv) in $\mathrm{DCM}(14 \mathrm{~mL}, 0.3 \mathrm{M})$ at $0{ }^{\circ} \mathrm{C}$. The reaction mixture was allowed to come to room temperature overnight. The suspension was extracted with bicarb (sat. $2 \times 10 \mathrm{~mL}$ ) and the organic layer dried over $\mathrm{MgSO}_{4}$. The residue was purified by silica gel chromatography (DCM:MeOH =96:4) to afford the desired product as an orange solid (910 mg, $2.84 \mathrm{mmol}, 71 \%$ ).

${ }^{1}$ H NMR $\left(400 \mathrm{MHz}\right.$, Methanol- $\left.d_{4}\right) \delta 8.71(\mathrm{~d}, \mathrm{~J}=2.1 \mathrm{~Hz}, 1 \mathrm{H}), 8.11(\mathrm{dd}, \mathrm{J}=8.4,2.5 \mathrm{~Hz}, 1 \mathrm{H})$, $7.70(\mathrm{~s}, 1 \mathrm{H}), 7.55(\mathrm{~d}, \mathrm{~J}=8.3 \mathrm{~Hz}, 1 \mathrm{H}), 4.31-4.18(\mathrm{~m}, 2 \mathrm{H}), 3.75-3.70(\mathrm{~m}, 2 \mathrm{H}) ;{ }^{13} \mathrm{C}$ NMR $(101$ MHz, Methanol- $\left.d_{4}\right) \delta 167.3,155.2,149.7,139.5,137.2,130.3,126.3,125.5,114.9,46.4,40.9$; $\mathbf{R}_{\mathbf{f}}(\mathrm{DCM}: \mathrm{MeOH}=96: 4): 0.08$; HR-MS (ESI) $\mathrm{m} / \mathrm{z}$ calculated for $\left[\mathrm{C}_{11} \mathrm{H}_{9} \mathrm{Cl}_{3} \mathrm{~N}_{4} \mathrm{ONa}\right]^{+}$ $\left([\mathrm{M}+\mathrm{Na}]^{+}\right): 340.9734$, measured: 340.9733.

Synthesis of methyl 2-bromo-5-phenylfuran (1p) and 2,4-dibromo-5-phenylfuran (1q)<smiles>c1coc(-c2ccco2)c1</smiles>

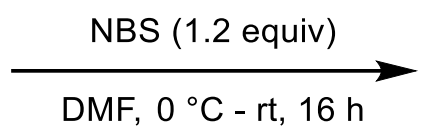<smiles>Brc1ccc(-c2ccccc2)o1</smiles>

$1 \mathrm{p}$<smiles>Brc1cc(Br)c(-c2ccccc2)o1</smiles>

$1 \mathrm{q}$

In an oven-dried Schlenk flask containing a magnetic stirring bar, methyl 2-phenylfuran ${ }^{44}$ (288 mg, $2.0 \mathrm{mmol}, 1.0$ equiv) was dissolved at $0{ }^{\circ} \mathrm{C}$ in DMF $(10 \mathrm{~mL})$ under argon. $\mathrm{N}$ Bromosuccinimide (NBS, $240 \mathrm{mg}, 1.8 \mathrm{mmol}, 1.2$ equiv) was added portion-wise and the reaction was allowed to warm to room temperature. The mixture was stirred for 16 and quenched with water. The aqueous layer was extracted with $\mathrm{Et}_{2} \mathrm{O}$ (3 times), the combined organic layers were dried over $\mathrm{MgSO}_{4}$ and the crude product was purified by column chromatography (pentane) to product $\mathbf{1 p}$ as a colorless oil (262 $\mathrm{mg}, 59 \%)$ and product $1 \mathbf{q}$ as a colorless oil (85.4 mg, 14\%).

Analytical Data for 1p:

${ }^{1}$ H NMR (400 MHz, Chloroform-d) $\delta 7.65-7.59(\mathrm{~m}, 2 \mathrm{H}), 7.42-7.35$ (m, 2H), $7.31-7.24$ $(\mathrm{m}, 1 \mathrm{H}), 6.61(\mathrm{~d}, J=3.4 \mathrm{~Hz}, 1 \mathrm{H}), 6.39(\mathrm{~d}, J=3.4 \mathrm{~Hz}, 1 \mathrm{H}) ;{ }^{13} \mathbf{C}$ NMR (101 MHz, Chloroformd) $\delta 156.1,130.1,128.9,127.9,123.6,121.6,113.5,107.4 ; \mathbf{R}_{\mathbf{f}}$ (pentane): 0.47; GC-MS (EI) $223.9\left(\left[\mathrm{M}^{+\cdot}\right], 20\right), 222.0$ (21), 115.1 (100), 89.0 (14), 63.0 (15), 51.0 (10).

Analytical Data for 1q:

${ }^{1}$ H NMR (400 MHz, Chloroform-d) $\delta 7.94-7.87$ (m, 2H), $7.47-7.40$ (m, 2H), 7.38 - 7.31 (m, 1H), 6.46 (s, 1H); ${ }^{13}$ C NMR (101 MHz, Chloroform-d) $\delta 151.5,129.0,128.7,128.5,125.5$,

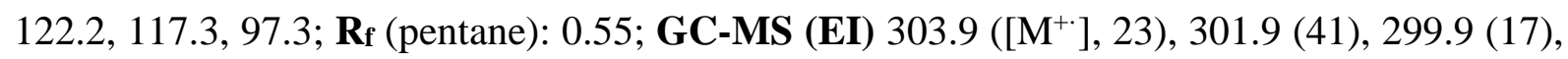
194.9 (100), 192.8 (80), 114.1 (86), 87.0 (19), 62.0 (29), 49.9 (19). 
Synthesis of 2-chloro-1-methyl-5-phenyl-1H-pyrrole (1r)<smiles>Cn1c(Cl)ccc1-c1ccccc1</smiles>

To a stirred solution of 1-methyl-2-phenyl- $1 H$-pyrrole $(157 \mathrm{mg}, 1.00 \mathrm{mmol}, 1.00$ equiv) in DMF $(5.9 \mathrm{~mL})$ was added NCS (140 mg, $1.05 \mathrm{mmol}, 1.05$ equiv) at $0{ }^{\circ} \mathrm{C}$. The solution was allowed to warm to room temperature and stirred overnight. The reaction was diluted with EtOAc and washed three times with water. The organic layer was dried over $\mathrm{Na}_{2} \mathrm{SO}_{4}$. Purification by column chromatography (pentane) yielded the desired product as a yellow solid.

${ }^{1}$ H NMR (300 MHz, Chloroform-d) $\delta 7.44-7.31(\mathrm{~m}, 5 \mathrm{H}), 6.18$ (d, $\left.J=3.8 \mathrm{~Hz}, 1 \mathrm{H}\right), 6.14$ (d, $J$ $=3.8 \mathrm{~Hz}, 1 \mathrm{H}), 3.58(\mathrm{~s}, 3 \mathrm{H}) ;{ }^{13} \mathbf{C}$ NMR $(75 \mathrm{MHz}$, Chloroform-d) $\delta 134.4,133.3,130.4,128.8$,

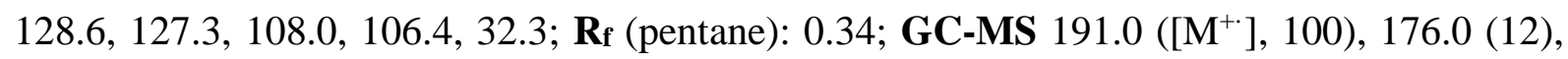
156.1 (14), 155.1 (10), 149.0 (11), 141.0 (14), 140.0 (36), 115.0 (42), 114.0 (12), 89.0 (12), $63.0(12)$.

Synthesis of 2,3,4-trichloro-1-methyl-5-phenyl-1H-pyrrole (1s)<smiles>Cn1c(Cl)c(Cl)c(Cl)c1-c1ccccc1</smiles>

To a stirred solution of 1-methyl-2-phenyl-1H-pyrrole (160 mg, $1.02 \mathrm{mmol}, 1.00$ equiv) in acetonitrile $(6.0 \mathrm{~mL})$ was added NCS $(449 \mathrm{mg}, 3.37 \mathrm{mmol}, 3.30$ equiv) and the resulting mixture was stirred overnight at $60{ }^{\circ} \mathrm{C}$. The reaction was allowed to warm to room temperature and was diluted with EtOAc. The mixture was washed three times with water and the organic layer was dried over $\mathrm{Na}_{2} \mathrm{SO}_{4}$. Purification by column chromatography (pentane) yielded the desired product as a yellow oil.

${ }^{1}$ H NMR (400 MHz, Chloroform-d) $\delta 7.50$ - $7.44(\mathrm{~m}, 2 \mathrm{H}), 7.44-7.39$ (m, 1H), 7.38 - 7.34 (m, 2H), 3.48 (s, 3H); ${ }^{13}$ C NMR (101 MHz, Chloroform-d) $\delta 130.4,129.5,129.5,128.8,128.8$, 114.4, 108.9, 108.4, 33.3; Rf (pentane): 0.38; GC-MS $258.9\left(\left[\mathrm{M}^{+\cdot}\right], 100\right), 224.0$ (13), 209.9 (15), 207.9 (21), 188.0 (10), 184.9 (20), 183.0 (31), 174.0 (14), 148.0 (13), 139.0 (10), 113.0 (19).

Synthesis of 2,3,4-tribromo-1-methyl-5-phenyl-1H-pyrrole (1t)<smiles>Cn1c(Br)c(Br)c(Br)c1-c1ccccc1</smiles>

To a stirred solution of 1-methyl-2-phenyl-1H-pyrrole (321 mg, $2.04 \mathrm{mmol}, 1.00$ equiv) in DMF $(12.0 \mathrm{~mL})$ was added NBS $\left(1.27 \mathrm{~g}, 7.14 \mathrm{mmol}, 3.50\right.$ equiv) at $0{ }^{\circ} \mathrm{C}$ and the resulting mixture was stirred overnight at $60^{\circ} \mathrm{C}$. The reaction was allowed to warm to room temperature and was diluted with EtOAc. The mixture was washed three times with water and the organic 
layer was dried over $\mathrm{Na}_{2} \mathrm{SO}_{4}$. Purification by column chromatography (pentane) yielded the desired product as a yellow oil.

${ }^{1}$ H NMR (300 MHz, Chloroform-d) $\delta 7.50$ - 7.41 (m, 3H), 7.37 - 7.32 (m, 2H), 3.52 (s, 3H); ${ }^{13}$ C NMR (75 MHz, Chloroform-d) $\delta$ 133.8, 130.7, 130.6, 128.9, 128.7, 104.7, 101.4, 98.4, 35.9; Rf (pentane): 0.23; GC-MS 394.8 (100), 393.9 (13), 392.8 (99), 390.8 ([M+] ], 35), 274.8 (13), 272.9 (23), 270.9 (12), 235.0 (14), 233.0 (15), 217.9 (11), 154.0 (19), 139.0 (28), 113.1 (25).

Synthesis of 5-chloro-4-phenylthiazol-2-amine (1ao)

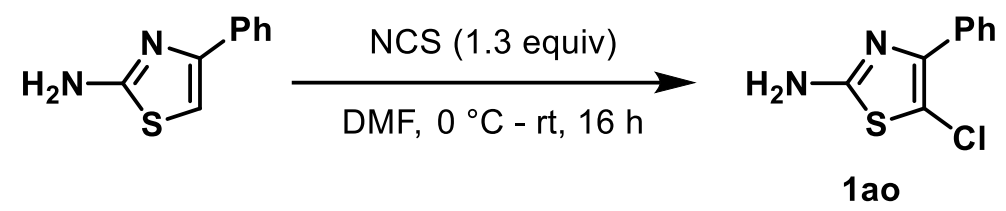

The compound was prepared according to a literature procedure. ${ }^{45}$ In an oven-dried Schlenk flask containing a magnetic stirring bar, 4-phenylthiazol-2-amine $(880 \mathrm{mg}, 5.0 \mathrm{mmol}$, 1.0 equiv) was dissolved in DMF $(25 \mathrm{~mL})$ under argon. $N$-Chlorosuccinimide (NCS, $868 \mathrm{mg}$, $6.5 \mathrm{mmol}, 1.3$ equiv) was added portion-wise at $0{ }^{\circ} \mathrm{C}$ and the reaction was allowed to warm to room temperature. The mixture was stirred for $16 \mathrm{~h}$, diluted with $\mathrm{Et}_{2} \mathrm{O}$ and washed with aqueous $\mathrm{NaHCO}_{3}$-solution and $\mathrm{H}_{2} \mathrm{O}$. The organic layer was dried over $\mathrm{Na}_{2} \mathrm{SO}_{4}$ and the solvent was removed under reduced pressure. The crude product was purified by column chromatography (pentane:EA = 3:1) to afford the title compound as a yellowish solid (798 mg, 76\%).

${ }^{1}$ H NMR (300 MHz, Chloroform-d) $\delta 7.88-7.81(\mathrm{~m}, 2 \mathrm{H}), 7.46-7.38(\mathrm{~m}, 2 \mathrm{H}), 7.38-7.30$ (m, 1H), 5.87 - 4.34 (b, 2H); ${ }^{13}$ C NMR (75 MHz, Chloroform-d) $\delta 163.3,145.2,132.8,128.5$, 128.4, 128.2 (one signal missing); Rf (pentane:EtOAc = 2:1): 0.31; HR-MS (ESI) $\mathrm{m} / \mathrm{z}$ calculated for $\left[\mathrm{C}_{9} \mathrm{H}_{7} \mathrm{ClN}_{2} \mathrm{SH}\right]^{+}\left([\mathrm{M}+\mathrm{H}]^{+}\right): 211.0091$, measured: 211.0106.

Synthesis of methyl 2,2,2-trifluoro- $N$-(4-mercaptophenyl)acetamide (2ai)

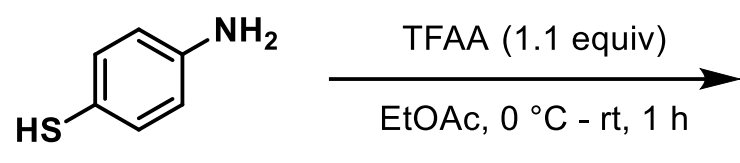<smiles>O=C(Nc1ccc(S)cc1)C(F)(F)F</smiles>

The compound was prepared according to a literature procedure. ${ }^{46}$ In an oven-dried Schlenk flask containing a magnetic stirring bar, methyl 4-aminothiophenol (1.25 g, $10.0 \mathrm{mmol}$, 1.0 equiv) was dissolved in EtOAc $(50 \mathrm{~mL})$ at $0{ }^{\circ} \mathrm{C}$ under argon. Trifluoroacetic anhydride (TFAA, $1.53 \mathrm{~mL}, 11.0 \mathrm{mmol}, 1.1$ equiv) was added dropwise and the reaction was allowed to warm to room temperature over $1 \mathrm{~h}$. The reaction was diluted with EtOAc $(50 \mathrm{~mL})$, washed with aqueous $\mathrm{HCl}$-solution $(1 \mathrm{M}, 50 \mathrm{~mL})$ and saturated $\mathrm{NaCl}$-solution $(50 \mathrm{~mL})$ and the organic layer was dried over $\mathrm{MgSO}_{4}$. The solvent was removed under reduced pressure and the crude product was purified by column chromatography $\left(\mathrm{CH}_{2} \mathrm{Cl}_{2}\right)$ to afford the title compound as a white solid (1.86 g, 84\%). 
${ }^{1}$ H NMR (400 MHz, Chloroform-d) $\delta 8.06$ (s, 1H), $7.48-7.35(\mathrm{~m}, 2 \mathrm{H}), 7.34-7.22(\mathrm{~m}, 2 \mathrm{H})$, 3.50 (s, 1H); ${ }^{13}$ C NMR (101 MHz, Chloroform-d) $\delta 155.0$ (q, $\left.J=37.3 \mathrm{~Hz}\right), 133.1,130.4,129.0$, 121.4, 115.8 (q, $J=288.5 \mathrm{~Hz}) ;{ }^{19} \mathbf{F}$ NMR $\left(376 \mathrm{MHz}\right.$, Chloroform-d) $\delta$-75.7; $\mathbf{R}_{\mathbf{f}}\left(\mathrm{CH}_{2} \mathrm{Cl}_{2}\right)$ : 0.33; HR-MS (ESI) $\mathrm{m} / \mathrm{z}$ calculated for $\left[\mathrm{C}_{8} \mathrm{H}_{6} \mathrm{NOSF}_{3} \mathrm{Na}\right]^{+}\left([\mathrm{M}+\mathrm{Na}]^{+}\right)$: 244.0014, measured: 244.0017 .

Synthesis of $N$-(2-mercaptoethyl)-5-((3aS,4S,6aR)-2-oxohexahydro-1H-thieno[3,4-d]imidazol4-yl)pentanamide (2aj)
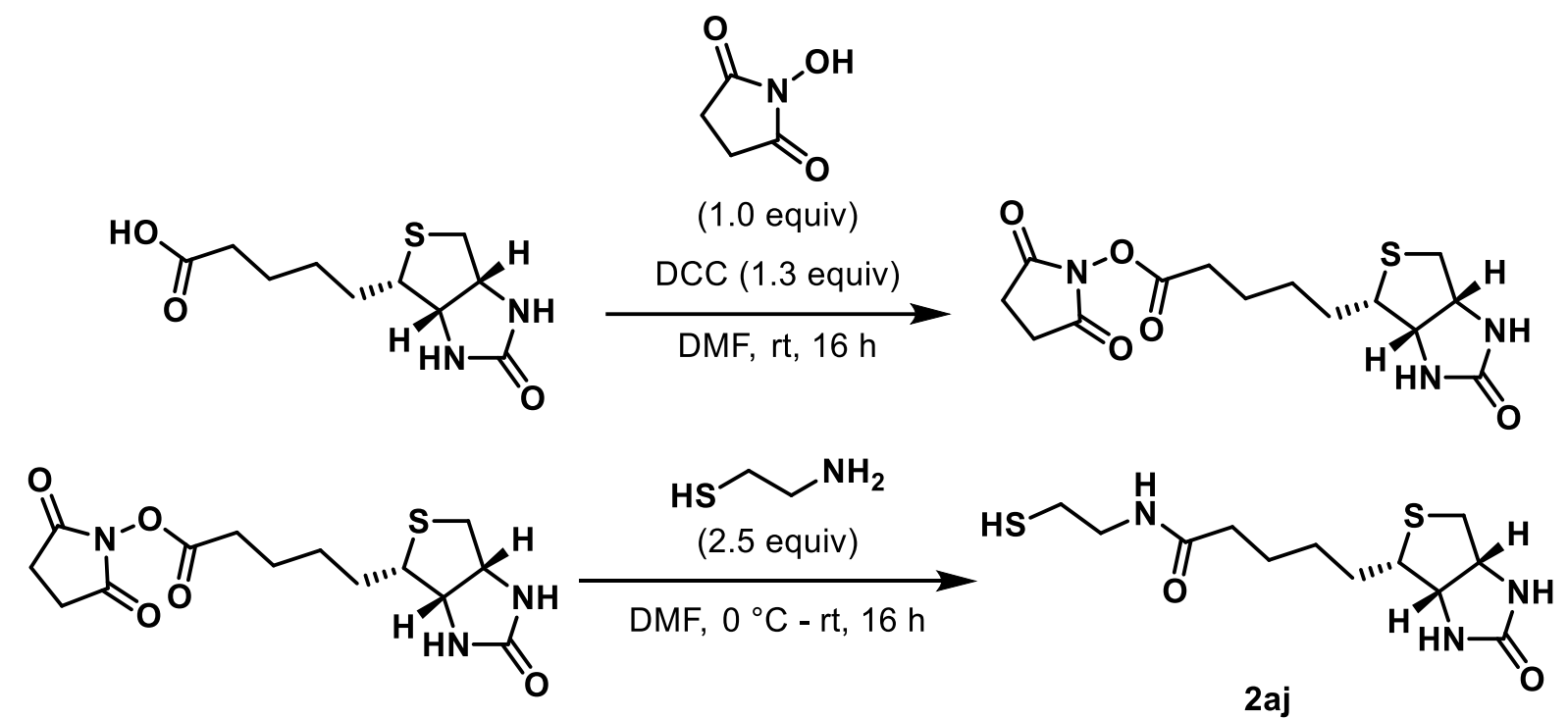

Compound 2aj was prepared in a two step procedure in analogy to a literature report. ${ }^{47}$ In an oven-dried Schlenk flask containing a magnetic stirring bar, biotin $(2.44 \mathrm{~g}, 10.0 \mathrm{mmol}$, 1.0 equiv) and $N$-hydroxysuccinimide ( $1.15 \mathrm{~g}, 10.0 \mathrm{mmol}, 1.0$ equiv) were dissolved in hot DMF (75 mL). Dicyclohexylcarbodiimide (DCC, $2.68 \mathrm{~g}, 13.0 \mathrm{mmol}, 1.3$ equiv) was added portion-wise and the mixture was allowed to stir at room temperature for $16 \mathrm{~h}$. The white precipitate was filtered off, the solvent was removed under reduced pressure and the crude product was triturated with $\mathrm{Et}_{2} \mathrm{O}$. The precipitate was isolated by filtration to give biotinyl- $\mathrm{N}-$ hydroxysuccinimide as a white solid (3.4 g, quant.) which was used in the next step without further purification.

In an oven-dried Schlenk flask containing a magnetic stirring bar, cysteamine $(772 \mathrm{mg}$, 10.0 mmol, 2.5 equiv) was dissolved in hot DMF (40 mL). The mixture was cooled to $0{ }^{\circ} \mathrm{C}$ and biotinyl- $N$-hydroxysuccinimide $(1.36 \mathrm{~g}, 4.0 \mathrm{mmol}, 1.0$ equiv) was added portion-wise over $45 \mathrm{~min}$. The reaction was allowed to warm to room temperature and was stirred for $16 \mathrm{~h}$. The solvent was removed under reduced pressure and the crude product was purified by column chromatography $\left(\mathrm{CH}_{2} \mathrm{Cl}_{2}: \mathrm{MeOH}=8: 1-7: 1\right)$ to give the title compound as a white solid (622 mg, 51\%).

${ }^{1} \mathbf{H}$ NMR (400 MHz, DMSO-d 6$) \delta 7.94(\mathrm{t}, J=5.7 \mathrm{~Hz}, 1 \mathrm{H}), 6.39(\mathrm{~d}, J=25.2 \mathrm{~Hz}, 2 \mathrm{H}), 4.36-$ $4.23(\mathrm{~m}, 1 \mathrm{H}), 4.19-4.04(\mathrm{~m}, 1 \mathrm{H}), 3.23-3.15(\mathrm{~m}, 2 \mathrm{H}), 3.09$ (ddd, $J=8.5,6.1,4.4 \mathrm{~Hz}, 1 \mathrm{H})$, $2.82(\mathrm{dd}, J=12.4,5.1 \mathrm{~Hz}, 1 \mathrm{H}), 2.57(\mathrm{~d}, J=12.4 \mathrm{~Hz}, 1 \mathrm{H}), 2.50(\mathrm{~m}, 2 \mathrm{H}$, overlapping with NMR solvent signal), $2.33(\mathrm{t}, J=8.0 \mathrm{~Hz}, 1 \mathrm{H}), 2.06(\mathrm{t}, J=7.4 \mathrm{~Hz}, 2 \mathrm{H}), 1.67-1.39(\mathrm{~m}, 4 \mathrm{H}), 1.37$ - 
$1.23(\mathrm{~m}, 2 \mathrm{H}) ;{ }^{13} \mathrm{C}$ NMR $(101 \mathrm{MHz}$, DMSO-d 6$) \delta 172.1,162.7,61.0,59.2,55.4,42.0,39.8$, 35.1, 28.2, 28.0, 25.2, 23.5; $\mathbf{R} \mathbf{f}\left(\mathrm{CH}_{2} \mathrm{Cl}_{2}: \mathrm{MeOH}=7: 1\right): 0.26$; HR-MS (ESI) $\mathrm{m} / \mathrm{z}$ calculated for $\left[\mathrm{C}_{12} \mathrm{H}_{21} \mathrm{~N}_{3} \mathrm{O}_{2} \mathrm{~S}_{2} \mathrm{Na}\right]^{+}\left([\mathrm{M}+\mathrm{Na}]^{+}\right): 326.0967$, measured: 326.0965 .

Synthesis of $\quad(3 \mathrm{R}, 5 \mathrm{~S}, 8 \mathrm{R}, 9 \mathrm{~S}, 10 \mathrm{~S}, 13 \mathrm{R}, 14 \mathrm{~S}, 17 \mathrm{R})-10,13-$ dimethyl-17-((R)-6-methylheptan-2yl)hexadecahydro-1H-cyclopenta[a]phenanthrene-3-thiol (2al)
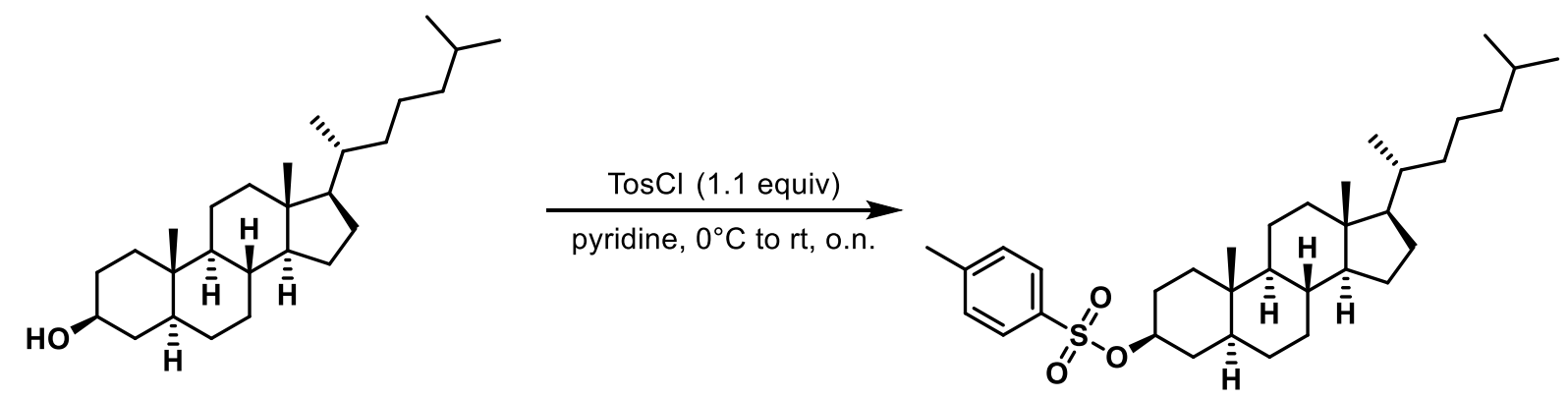

$\operatorname{KSAc}(2.2$ equiv) DMF, $85^{\circ} \mathrm{C}, 3 \mathrm{~h}$
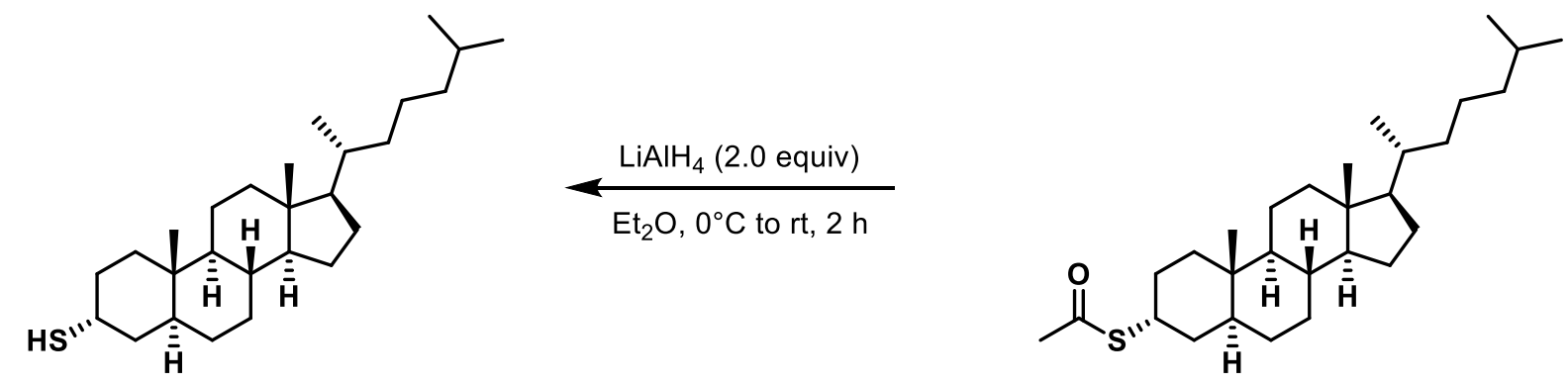

Cholestanol (2.72 g, $7.0 \mathrm{mmol}, 1.0$ equiv) was dissolved in dry pyridine $(7 \mathrm{~mL}, 1.0 \mathrm{M})$ and cooled to $0^{\circ} \mathrm{C}$. p-Toluenesulfonyl chloride $(1.47 \mathrm{~g}, 7.7 \mathrm{mmol}, 1.1$ equiv) was added to the reaction mixture and the solution was stirred at room temperature overnight. Pentane $(25 \mathrm{~mL})$ was added, the precipitate was filtered off and the washed with pentane. The combined organic layers were extracted with aq. $\mathrm{HCl}(4 \mathrm{M}, 2 \times 20 \mathrm{~mL})$ and dried over $\mathrm{MgSO}_{4}$. The solvent was removed under reduced pressure and the residue was purified by column chromatography (pentane/EtOAc 95:5) to afford the tosylated cholestanol as white solid (3.45 g, $6.4 \mathrm{mmol}$, $91 \%)$.

The tosylated cholestanol ( $3.42 \mathrm{~g}, 6.3 \mathrm{mmol}, 1.0$ equiv) was dissolved in dry DMF (18 mL, $0.2 \mathrm{M})$ and $\mathrm{KSAc}(1.60 \mathrm{~g}, 14 \mathrm{mmol}, 2.2$ equiv) was added at room temperature. The reaction mixture was stirred for $3 \mathrm{~h}$ at $85^{\circ} \mathrm{C}$ and cooled to come to room temperature afterwards. $\mathrm{Et}_{2} \mathrm{O}$ $(30 \mathrm{~mL}$ ) was added to the solution and the reaction mixture was extracted with bicarb (half sat. $2 \times 50 \mathrm{~mL}$ ). The organic phase was dried over $\mathrm{MgSO}_{4}$, the solvent was removed under reduced pressure and the residue was purified by column chromatography (pentane/EtOAc 96:4) to afford the title compound as brown solid $(2.05 \mathrm{~g}, 4.59 \mathrm{mmol}, 73 \%)$.

The thioacetate ( $4.59 \mathrm{mmol}, 2.05 \mathrm{~g}, 1.0$ equiv) was dissolved in dry diethyl ether $(30 \mathrm{~mL}, 0.2 \mathrm{M})$ and cooled to $0{ }^{\circ} \mathrm{C}$. $\mathrm{LiAlH}_{4}(348 \mathrm{mg}, 9.16 \mathrm{mmol}, 2.0$ equiv ) was added carefully and the solution was allowed to come to room temperature and stirred for additional $2 \mathrm{~h}$. Diethyl ether 
$(30 \mathrm{~mL}$ ) was added to the reaction mixture followed by the carful addition of $\mathrm{HCl}$ (aq. $2 \mathrm{~N}$, $30 \mathrm{~mL}$ ) at $0{ }^{\circ} \mathrm{C}$. The layers were separated and the aqueous phase was washed with diethyl ether $(20 \mathrm{~mL})$. The combined organic layers were washed with bicarb $(20 \mathrm{~mL})$ and dried over $\mathrm{MgSO}_{4}$. The solvent was removed under reduced pressure and the residue was purified by column chromatography (pentane) to afford the title compound as white solid (1.62 g, $4.0 \mathrm{mmol}, 88 \%$ ).

${ }^{1} \mathbf{H}$ NMR (300 MHz, Chloroform-d) $\delta 3.77-3.39(\mathrm{~m}, 1 \mathrm{H}), 2.03-1.90$ (m, 2H), $1.87-0.71$ (m, 42H), 0.64 (s, 3H); ${ }^{13}$ C NMR (75 MHz, Chloroform-d) $\delta$ 56.7, 56.4, 54.3, 42.7, 40.2, 39.7, 39.7, 37.0, 36.6, 36.6, 36.3, 36.0, 35.6, 32.5, 32.0, 30.0, 28.5, 28.4, 28.2 24.3, 24.0, 23.0, 22.7, 20.9, 18.8, 12.2, 12.0; $\mathbf{R}_{\mathbf{f}}$ (pentane): 0.35 .

Synthesis of methyl $N^{2}$-(tert-butoxycarbonyl)- $N^{4}$-(2-mercaptoethyl)- $L$-asparaginyl- $L$ phenylalaninate (2am)<smiles>COC(=O)[C@H](Cc1ccccc1)NC(=O)[C@H](Cc1ccccc1)NC(=O)OC(C)(C)C</smiles>

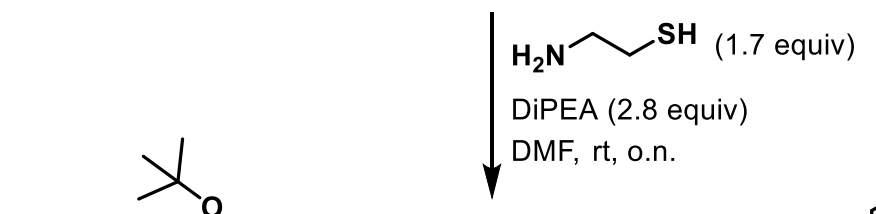<smiles>COC(=O)[C@H](Cc1ccccc1)NC(=O)C(CC(=O)NCCSSCCNC(=O)C[C@H](NC(=O)OC(C)(C)C)C(=O)N[C@@H](Cc1ccccc1)C(=O)OC)NC(=O)OC(C)(C)C</smiles>

$\mathrm{PPh}_{3}(1.05$ equiv) $\mathrm{HOAC} / \mathrm{NaOAC}$ $\mathrm{H}_{2} \mathrm{O} / \mathrm{MeOH}, 60^{\circ} \mathrm{C}, 1.5 \mathrm{~h}$<smiles>COC(=O)[C@H](Cc1ccccc1)NC(=O)[C@H](CC(=O)NCCS)NC(=O)OC(C)(C)C</smiles> 
Aspartame (2.94 g, $10 \mathrm{mmol}, 1.0$ equiv) was dissolved in a bisphasic mixture of $\mathrm{H}_{2} \mathrm{O}$ and 1,4dioxane (1:1, $40 \mathrm{~mL})$. $\mathrm{NEt}_{3}(3.89 \mathrm{~mL}, 28 \mathrm{mmol}, 2.8$ equiv $)$ and di-tert-butyl dicarbonate $(2.62 \mathrm{~g}$, $12 \mathrm{mmol}, 1.2$ equiv) were added afterwards and the resulting mixture was stirred at room temperature for $18 \mathrm{~h}$. The biphasic mixture was partitioned between EtOAc $(40 \mathrm{~mL})$ and a citric acid solution (aq., $10 \mathrm{wt} . \%, 40 \mathrm{~mL})$. The aqueous layer was extracted with EtOAc $(2 \mathrm{x} 20 \mathrm{~mL})$ and the combined organic layers were washed with brine and dried over $\mathrm{MgSO}_{4}$. After removal of the volatiles the Boc-procected aspartame was obtained as white solid (2.85 g, $7.22 \mathrm{mmol}$, $72 \%)$.

Boc-protected aspartame ( $2.37 \mathrm{~g}, 6.0 \mathrm{mmol}, 1.0$ equiv) was dissolved in dry DMF (50 mL). $O$ (7-Azabenzotriazole-1-yl)- $N, N, N, N$ '-tetramethyluronium hexafluorophosphate (HATU, 2.51g, $6.6 \mathrm{mmol}, 1.1$ equiv) was added and the solution was stirred for $10 \mathrm{~min}$ at room temperature. Cysteamin (770 mg, $10 \mathrm{mmol}, 1.7$ equiv) was added afterwards and the solution was stirred for $20 \mathrm{~min}$ followed by the addition of $N, N$-diisopropylethylamine $(2.97 \mathrm{~mL}, 17 \mathrm{mmol}, 2.8$ equiv). After stirring over-night, brine $(500 \mathrm{~mL})$ was added and the reaction was cooled down to $4{ }^{\circ} \mathrm{C}$. The precipitate was filtered off and identified to be the disulfide of the desired product $(920 \mathrm{mg}$, $1.0 \mathrm{mmol}, 34 \%$ ). It was found that the commercially obtained cysteamin was actually predominantly the disulfide instead of the desired thiol.

The disulfide was cleaved by dissolving dimethyl (2S,5S,18S,21S)-2,21-dibenzyl-5,18bis((tert-butoxycarbonyl)amino)-4,7,16,19-tetraoxo-11,12-dithia-3,8,15,20-

tetraazadocosanedioate $(835 \mathrm{mg}, 0.92 \mathrm{mmol}, \quad 1.0$ equiv), triphenylphosphine (254 mg, $0.97 \mathrm{mmol}, 1.05$ equiv) and $\mathrm{NaOAc}(30 \mathrm{mg}, 0.37 \mathrm{mmol}, 0.40$ equiv) in $\mathrm{MeOH}(7.5 \mathrm{~mL})$ and water $(1.8 \mathrm{~mL})$ under argon. Acetic acid $(95 \mu \mathrm{L}, 1.66 \mathrm{mmol}, 1.05$ equiv) was added via syringe and the mixture was heated to $60{ }^{\circ} \mathrm{C}$ for $1.5 \mathrm{~h} . \mathrm{H}_{2} \mathrm{O}(30 \mathrm{~mL})$ was added and the mixture was extracted with $\mathrm{CH}_{2} \mathrm{Cl}_{2}$ (3 times). The combined organic layers were dried over $\mathrm{Na}_{2} \mathrm{SO}_{4}$ and the solvent was removed under reduced pressure. The crude product was purified by column chromatography (pentane:EtOAc $=2: 3-$ EtOAc) to afford the title compound as a white solid (301 mg, 36\%).

${ }^{1} \mathbf{H}$ NMR (300 MHz, Chloroform-d) $\delta 7.34-7.14(\mathrm{~m}, 4 \mathrm{H}), 7.13-7.03(\mathrm{~m}, 2 \mathrm{H}), 6.44-6.27$ $(\mathrm{m}, 1 \mathrm{H}), 6.17-5.94(\mathrm{~m}, 1 \mathrm{H}), 4.70(\mathrm{dt}, J=7.7,6.1 \mathrm{~Hz}, 1 \mathrm{H}), 4.48-4.33(\mathrm{~m}, 1 \mathrm{H}), 3.62(\mathrm{~s}, 3 \mathrm{H})$, $3.34(\mathrm{qd}, J=6.4,1.4 \mathrm{~Hz}, 2 \mathrm{H}), 3.12-2.91(\mathrm{~m}, 2 \mathrm{H}), 2.77(\mathrm{dd}, J=15.2,4.0 \mathrm{~Hz}, 1 \mathrm{H}), 2.63-2.43$ $(\mathrm{m}, 3 \mathrm{H}), 1.45-1.30$ (m, 10H); ${ }^{13} \mathbf{C}$ NMR (75 MHz, Chloroform-d) $\delta$ 171.6, 171.4, 171.2, 155.9, $135.9,129.4,128.7,127.3,80.5,53.8,52.4,51.2,42.6,38.0,37.5,28.4,24.5 ; \mathbf{R}_{\mathbf{f}}$ (pentane:EtOAc = 3:7): 0.26; HR-MS (ESI) m/z calculated for $\left[\mathrm{C}_{21} \mathrm{H}_{31} \mathrm{~N}_{3} \mathrm{O}_{6} \mathrm{SNa}\right]^{+}\left([\mathrm{M}+\mathrm{Na}]^{+}\right)$: 476.1826, measured: 476.1837. 


\subsection{Optimization Studies}

Unless otherwise noted, 4,5-dichloro-1-methyl- $1 H$-imidazole (1a, $15.1 \mathrm{mg}, 0.10 \mathrm{mmol}$, 1.0 equiv) and $\left[\operatorname{Ir}\left(\mathrm{dFCF}_{3} \text { ppy }\right)_{2}(\mathrm{dtbbpy})\right] \mathrm{PF}_{6}(2.2 \mathrm{mg}, 0.002 \mathrm{mmol}, 2.0 \mathrm{~mol} \%)$ were weighed into a Schlenk tube containing a magnetic stirring bar. The tube was evacuated and backfilled with argon three times. DMA $(1 \mathrm{~mL}, 0.1 \mathrm{M})$ was added, followed by methyl 3mercaptopropanoate $(\mathbf{2 a}, 22.2 \mu \mathrm{L}, 0.20 \mathrm{mmol}, 2.0$ equiv). The reaction was stirred under irradiation with $455 \mathrm{~nm}$ LEDs for $16 \mathrm{~h}$ at room temperature. Mesitylene (13.8 $\mu \mathrm{L}, 0.1 \mathrm{mmol}$, 1.0 equiv) was added as an internal standard and an aliquot $(100 \mu \mathrm{L})$ of the crude reaction mixture was filtered over a plug of silica (elution with EtOAc). The yield was determined via calibrated GC-FID.

For unpolar solvents, a basic work-up was performed after addition of Mesitylene (1.0 equiv). The crude mixture was diluted with EtOAc. $\mathrm{NaHCO}_{3}$-solution (sat., $3 \mathrm{~mL}$ ) and water $(3 \mathrm{~mL})$ were added and the layers were separated. An aliquot $(100 \mu \mathrm{L})$ of the organic layer was filtered over a plug of silica (elution with EtOAc) and the yield was determined via calibrated GC-FID. 
Table S1. Optimization studies.

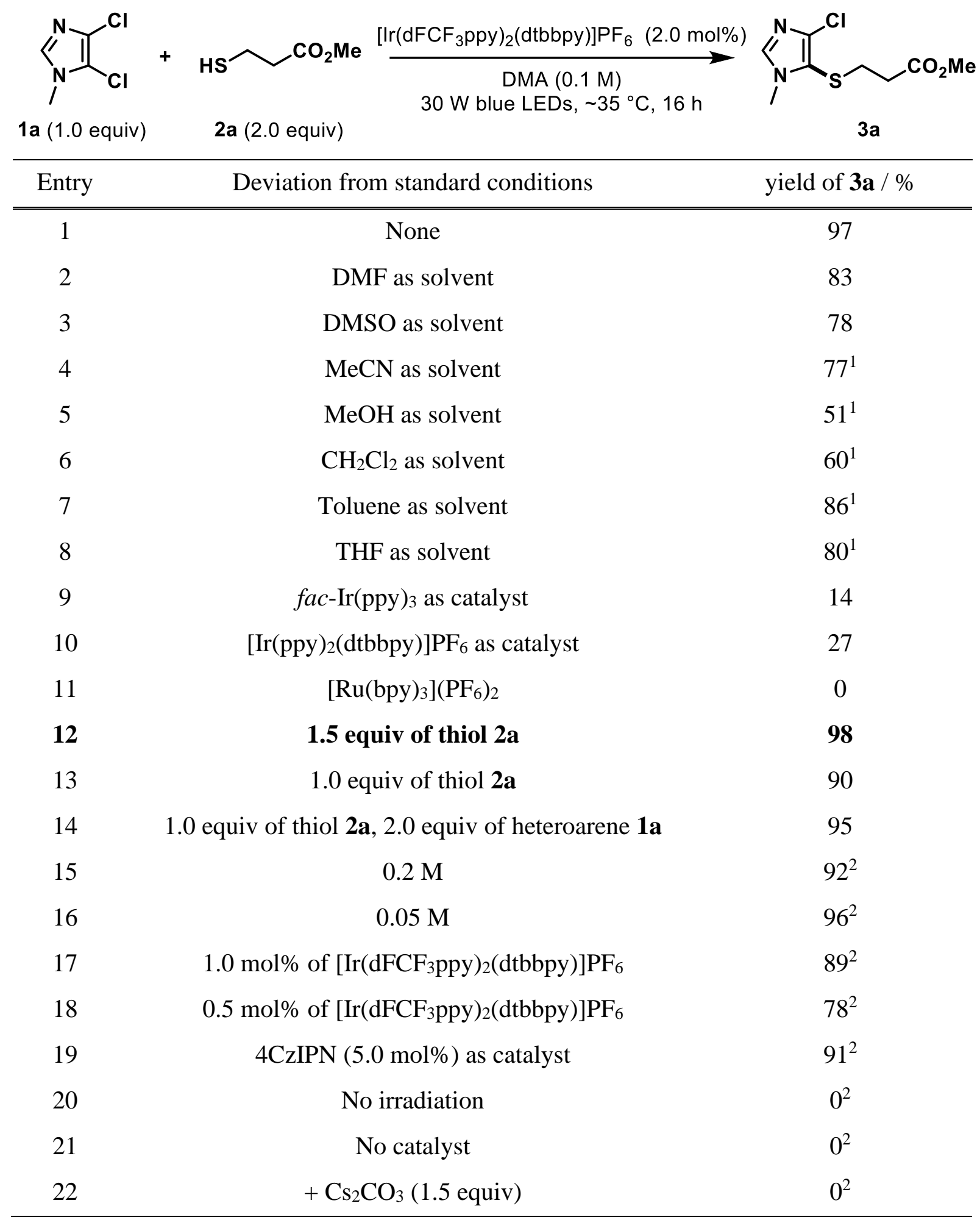

${ }^{1}$ An aqueous work-up was performed. ${ }^{2}$ Using 1.5 equiv of thiol $2 \mathbf{a}$. 


\subsection{Sensitivity Assessment}

The sensitivity assessment was carried out according to Glorius et al. ${ }^{22}$ Yields were determined by analyzing an aliquot $(100 \mu \mathrm{L})$ of the crude reaction mixture via calibrated GC-FID using mesitylene as an internal standard. For experimental details see below and Table S2. The results are displayed in Table S3 and Figure S4.
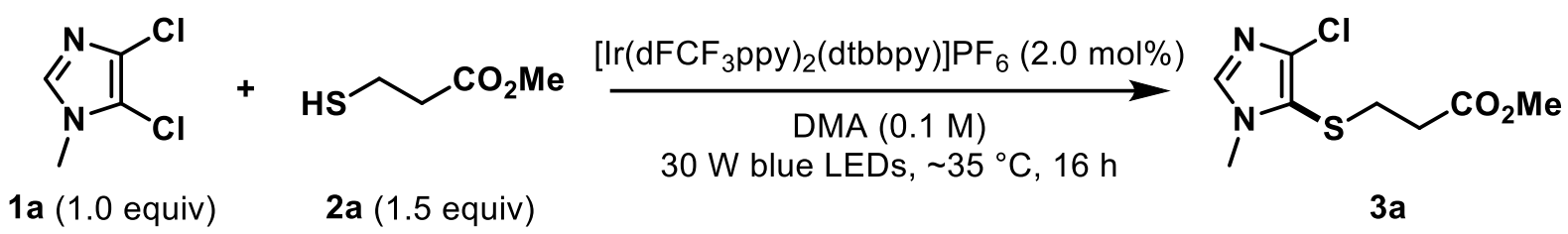

Standard conditions: $n=0.3 \mathrm{mmol}, c=0.1 \mathrm{M}, V=3.0 \mathrm{~mL}$, inert atmosphere, $T=35^{\circ} \mathrm{C}, d=$ $8 \mathrm{~cm}$.

Stock solution: $n=3.6 \mathrm{mmol}, c=0.111 \mathrm{M}, V=32.4 \mathrm{~mL}, 4,5$-dichloro-1-methyl- $1 H$-imidazole: $544 \mathrm{mg}$, methyl 3-mercaptopropanoate: $599 \mu \mathrm{L},\left[\operatorname{Ir}\left(\mathrm{dFCF}_{3} \text { ppy }\right)_{2}(\mathrm{dtbbpy})\right] \mathrm{PF}_{6}: 80.4 \mathrm{mg}$, DMA: $32.4 \mathrm{~mL}$, mesitylene: $497 \mu \mathrm{L}$ (1.0 equiv).

Table S2. Preparation of the sensitivity assessment.

\begin{tabular}{cll}
\hline Entry & Experiment & Preparation \\
\hline \hline 1 & High $c$ & $2.7 \mathrm{~mL}$ stock sol. \\
2 & Low $c$ & $2.7 \mathrm{~mL}$ stock sol. $+0.6 \mathrm{~mL}$ DMA \\
3 & High $\mathrm{H}_{2} \mathrm{O}$ & $2.7 \mathrm{~mL}$ stock sol. $+0.3 \mathrm{~mL} \mathrm{DMA}+30 \mu \mathrm{L} \mathrm{H}_{2} \mathrm{O}$ \\
4 & Low $\mathrm{O}_{2}$ & $2.7 \mathrm{~mL}$ stock sol. $+0.3 \mathrm{~mL} \mathrm{DMA}+$ degassed \\
5 & High $\mathrm{O}_{2}$ & $2.7 \mathrm{~mL}$ stock sol. $+0.3 \mathrm{~mL} \mathrm{DMA}+30 \mathrm{~mL}$ air \\
6 & Low $T$ & $2.7 \mathrm{~mL}$ stock sol. $+0.3 \mathrm{~mL} \mathrm{DMA,} T=15^{\circ} \mathrm{C}$ \\
7 & High $T$ & $2.7 \mathrm{~mL}$ stock sol. $+0.3 \mathrm{~mL} \mathrm{DMA,} T=55^{\circ} \mathrm{C}$ \\
8 & Low $I$ & $2.7 \mathrm{~mL}$ stock sol. $+0.3 \mathrm{~mL} \mathrm{DMA,} d=32 \mathrm{~cm}$ \\
9 & High $I$ & $2.7 \mathrm{~mL}$ stock sol. $+0.3 \mathrm{~mL} \mathrm{DMA}, d=2 \mathrm{~cm}$ \\
10 & Control & $2.7 \mathrm{~mL}$ stock sol. $+0.3 \mathrm{~mL} \mathrm{DMA}$ \\
11 & Big Scale & see section 2.4 \\
\hline
\end{tabular}

Table S3. Results of the sensitivity assessment.

\begin{tabular}{llcc}
\hline Entry & Experiment & Yield / \% & Relative Deviation / \% \\
\hline \hline 1 & High $c$ & 99 & 1 \\
2 & Low $c$ & 98 & 0 \\
3 & High $\mathrm{H}_{2} \mathrm{O}$ & 95 & -3
\end{tabular}




\begin{tabular}{lllc}
4 & Low $\mathrm{O}_{2}$ & 98 & 0 \\
5 & High $\mathrm{O}_{2}$ & 86 & -13 \\
6 & Low $T$ & 98 & 0 \\
7 & High $T$ & 97 & -1 \\
8 & Low $I$ & 99 & 1 \\
9 & High $I$ & 99 & 1 \\
10 & Control & 98 & \\
11 & Big Scale & 99 & 1 \\
\hline
\end{tabular}

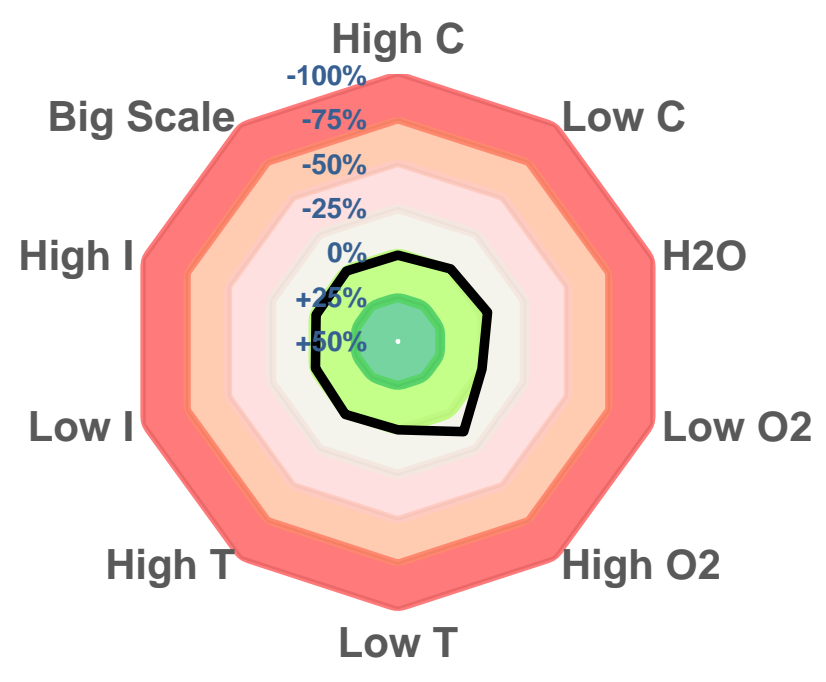

Figure S4. Radar diagram for the results of the sensitivity assessment (see Table S3).

\subsection{Reaction Scale-up}

4,5-Dichloro-1-methyl- $1 H$-imidazole $\quad(906 \mathrm{mg}, \quad 6.0 \mathrm{mmol}, \quad 1.0$ equiv) and $\left[\operatorname{Ir}\left(\mathrm{dFCF}_{3} \text { ppy }\right)_{2}(\mathrm{dtbbpy})\right] \mathrm{PF}_{6}(135 \mathrm{mg}, 0.12 \mathrm{mmol}, 2.0 \mathrm{~mol} \%)$ were weighed into a Schlenk tube containing a magnetic stirring bar. The tube was evacuated and backfilled with argon three times. DMA $(60 \mathrm{~mL}, 0.1 \mathrm{M})$ was added, followed by methyl 3-mercaptopropanoate $(997 \mu \mathrm{L}$, $9.0 \mathrm{mmol}, 1.5$ equiv). The reaction was stirred under irradiation with $455 \mathrm{~nm}$ LEDs for $16 \mathrm{~h}$ at room temperature. Mesitylene ( $829 \mu \mathrm{L}, 6.0 \mathrm{mmol}, 1.0$ equiv) was added as an internal standard and an aliquot $(100 \mu \mathrm{L})$ of the crude reaction mixture was filtered over a plug of silica (elution with EtOAc). The yield was determined via calibrated GC-FID. The crude mixture was further diluted with EtOAc. $\mathrm{NaHCO}_{3}$-solution (sat., $200 \mathrm{~mL}$ ) and water $(200 \mathrm{~mL}$ ) were added, the layers were separated, and the organic layer was washed with water $(2 \times 300 \mathrm{~mL})$ and dried over $\mathrm{Na}_{2} \mathrm{SO}_{4}$. The solvent was removed under reduced pressure and the crude product was purified by column chromatography (EtOAc) over silica gel to afford the pure product as a yellowish oil $(1.40 \mathrm{~g}, 99 \%)$.

For characterization data, see section 2.5. 


\subsection{Substrate Scope and Characterization Data}<smiles>[X][Y]1c([Z])sc([Y])c1[X]</smiles>

1 (1.0 equiv)

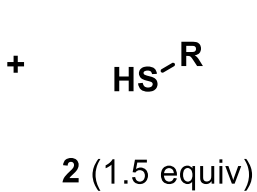

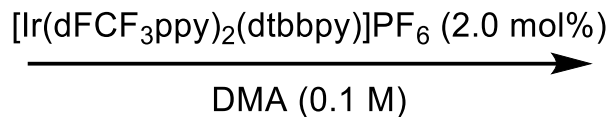

$30 \mathrm{~W}$ blue LEDs, $\sim 35^{\circ} \mathrm{C}, 16 \mathrm{~h}$

\section{General Procedure A}

Unless otherwise noted, the corresponding heteroarene $(0.30 \mathrm{mmol}, 1.0$ equiv $)$ and $\left[\operatorname{Ir}\left(\mathrm{dFCF}_{3} \text { ppy }\right)_{2}(\mathrm{dtbbpy})\right] \mathrm{PF}_{6}(6.7 \mathrm{mg}, 0.006 \mathrm{mmol}, 2.0 \mathrm{~mol} \%)$ were weighed into a Schlenk tube containing a magnetic stirring bar. If solid, the respective thiol ( $0.45 \mathrm{mmol}, 1.5$ equiv) was added analogously. The tube was evacuated and backfilled with argon three times. DMA (3 mL, $0.1 \mathrm{M})$ was added, followed by the respective thiol ( $0.45 \mathrm{mmol}, 1.5$ equiv) (if liquid). The reaction was stirred under irradiation with $455 \mathrm{~nm}$ LEDs for $16 \mathrm{~h}$ at room temperature. The crude mixture was diluted with EtOAc. $\mathrm{NaHCO}_{3}$-solution (sat., $10 \mathrm{~mL}$ ) and water $(10 \mathrm{~mL}$ ) were added, the layers were separated, and the organic layer was washed with water $(2 \times 20 \mathrm{~mL})$ and dried over $\mathrm{Na}_{2} \mathrm{SO}_{4}$. The solvent was removed under reduced pressure and the crude product was purified by column chromatography over silica gel to afford analytically pure products.

\section{General Procedure B}

Unless otherwise noted, the corresponding heteroarene $(0.45 \mathrm{mmol}, 1.5$ equiv), $\left[\operatorname{Ir}\left(\mathrm{dFCF}_{3} \mathrm{ppy}\right)_{2}(\mathrm{dtbbpy})\right] \mathrm{PF}_{6} \quad(6.7 \mathrm{mg}, \quad 0.006 \mathrm{mmol}, \quad 2.0 \mathrm{~mol} \%)$ and $\mathrm{K}_{2} \mathrm{HPO}_{4} \quad(63 \mathrm{mg}$, $0.36 \mathrm{mmol}, 1.2$ equiv) were weighed into a Schlenk tube containing a magnetic stirring bar. If solid, the respective thiol $(0.30 \mathrm{mmol}, 1.0$ equiv) was added analogously. The tube was evacuated and backfilled with argon three times. DMA ( $3 \mathrm{~mL}, 0.1 \mathrm{M})$ was added, followed by the respective thiol ( $0.30 \mathrm{mmol}, 1.0$ equiv) (if liquid). The reaction was stirred under irradiation with $455 \mathrm{~nm}$ LEDs for $16 \mathrm{~h}$ at room temperature. The crude mixture was diluted with EtOAc. $\mathrm{NaHCO}_{3}$-solution (sat., $\left.10 \mathrm{~mL}\right)$ and water $(10 \mathrm{~mL})$ were added, the layers were separated, and the organic layer was washed with water $(2 \times 20 \mathrm{~mL})$ and dried over $\mathrm{Na}_{2} \mathrm{SO}_{4}$. The solvent was removed under reduced pressure and the crude product was purified by column chromatography over silica gel to afford analytically pure products.

\section{General Procedure C}

Unless otherwise noted, the corresponding heteroarene $(0.30 \mathrm{mmol}, 1.0$ equiv) (if liquid) and $\left[\operatorname{Ir}\left(\mathrm{dFCF}_{3} \text { ppy }\right)_{2}(\mathrm{dtbbpy})\right] \mathrm{PF}_{6}(6.7 \mathrm{mg}, 0.006 \mathrm{mmol}, 2.0 \mathrm{~mol} \%)$ were weighed into a Schlenk tube containing a magnetic stirring bar. If solid, the respective thiol $(0.45 \mathrm{mmol}, 1.5$ equiv $)$ was added analogously. The tube was evacuated and backfilled with argon three times. DMA (3 mL, $0.1 \mathrm{M})$ was added, followed by the respective heteroarene $(0.30 \mathrm{mmol}, 1.0$ equiv $)$ and thiol ( $0.45 \mathrm{mmol}, 1.5$ equiv) (if liquid). Pyridine ( $37 \mu \mathrm{L}, 0.45 \mathrm{mmol}, 1.5$ equiv) was added and the reaction was stirred under irradiation with $455 \mathrm{~nm}$ LEDs for $16 \mathrm{~h}$ at room temperature. The crude mixture was diluted with EtOAc. $\mathrm{NaHCO}_{3}$-solution (sat., $10 \mathrm{~mL}$ ) and water $(10 \mathrm{~mL})$ were added, the layers were separated, and the organic layer was washed with water $(2 \times 20 \mathrm{~mL})$ and 
dried over $\mathrm{Na}_{2} \mathrm{SO}_{4}$. The solvent was removed under reduced pressure and the crude product was purified by column chromatography over silica gel to afford analytically pure products.

Methyl 3-((4-chloro-1-methyl-1H-imidazol-5-yl)thio)propanoate (3a)<smiles>COC(=O)CCSc1c(Cl)ncn1C</smiles>

Prepared from 4,5-dichloro-1-methyl-1H-imidazole $(45.3 \mathrm{mg}, 0.30 \mathrm{mmol})$ and methyl 3mercaptopropanoate $(49.9 \mu \mathrm{L}, 0.45 \mathrm{mmol})$ following general procedure A. The pure product was obtained after column chromatography (EtOAc) as a yellowish oil (70.0 mg, 99\%).

${ }^{1} \mathbf{H}$ NMR (400 MHz, Chloroform-d) $\delta 7.44(\mathrm{~s}, 1 \mathrm{H}), 3.63(\mathrm{~d}, \mathrm{~J}=0.6 \mathrm{~Hz}, 3 \mathrm{H}), 3.60$ (s, 3H), 2.86 (t, $J=7.0 \mathrm{~Hz}, 2 \mathrm{H}), 2.54$ (t, J = 7.0 Hz, 2H); ${ }^{13} \mathbf{C ~ N M R}(101 \mathrm{MHz}$, Chloroform-d) $\delta$ 171.7, 137.9, 137.7, 117.0, 51.9, 34.5, 32.8, 30.8; Rf (EtOAc): 0.30; HR-MS (ESI) m/z calculated for $\left[\mathrm{C}_{8} \mathrm{H}_{11} \mathrm{~N}_{2} \mathrm{O}_{2} \mathrm{SClNa}\right]^{+}\left([\mathrm{M}+\mathrm{Na}]^{+}\right): 257.0122$, measured: 257.0128 .

Methyl 3-((4-bromo-1-methyl-1H-imidazol-5-yl)thio)propanoate (3b)<smiles>COC(=O)CCSc1c(Br)ncn1C</smiles>

Prepared from 4,5-dibromo-1-methyl- $1 \mathrm{H}$-imidazole $(72 \mathrm{mg}, 0.30 \mathrm{mmol})$ and methyl 3mercaptopropanoate $(49.9 \mu \mathrm{L}, 0.45 \mathrm{mmol})$ following general procedure A. The pure product was obtained after column chromatography (pentane:EtOAc $=1: 2-$ EtOAc) as a yellowish oil (79.5 mg, 95\%).

${ }^{1}$ H NMR (400 MHz, Chloroform-d) $\delta 7.50(\mathrm{~s}, 1 \mathrm{H}), 3.67(\mathrm{~s}, 3 \mathrm{H}), 3.62(\mathrm{~s}, 3 \mathrm{H}), 2.89$ (t, J = 7.0 $\mathrm{Hz}, 2 \mathrm{H}), 2.56(\mathrm{t}, J=7.0 \mathrm{~Hz}, 2 \mathrm{H}) ;{ }^{13} \mathrm{C}$ NMR (101 MHz, Chloroform-d) $\delta 171.8,139.3,126.0$, 120.3, 52.0, 34.5, 33.0, 30.9; $\mathbf{R}_{\mathbf{f}}$ (EtOAc): 0.28; HR-MS (ESI) $\mathrm{m} / \mathrm{z}$ calculated for $\left[\mathrm{C}_{8} \mathrm{H}_{11} \mathrm{~N}_{2} \mathrm{O}_{2} \mathrm{SBrNa}\right]^{+}\left([\mathrm{M}+\mathrm{Na}]^{+}\right): 302.9596$, measured: 302.9607.

Methyl 3-((1-ethyl-2-methyl-1H-imidazol-5-yl)thio)propanoate (3c)

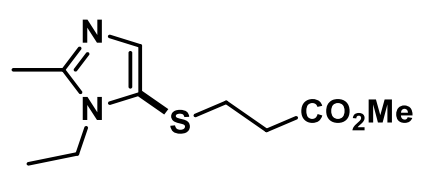

Prepared from 5-chloro-1-ethyl-2-methyl- $1 H$-imidazole $(43.4 \mathrm{mg}, 0.30 \mathrm{mmol})$ and methyl 3mercaptopropanoate $(49.9 \mu \mathrm{L}, 0.45 \mathrm{mmol})$ following general procedure A. The pure product was obtained after column chromatography (EtOAc - EtOAc: $\mathrm{MeOH}=33: 1)$ as a yellowish oil (39.0 mg, 57\%).

${ }^{1}$ H NMR (400 MHz, Chloroform-d) $\delta 7.07$ (s, 1H), 4.00 (q, J=7.2 Hz, 2H), 3.65 (s, 3H), 2.77 (t, $J=7.2 \mathrm{~Hz}, 2 \mathrm{H}), 2.55(\mathrm{t}, J=7.1 \mathrm{~Hz}, 2 \mathrm{H}), 2.40(\mathrm{~s}, 3 \mathrm{H}), 1.27(\mathrm{t}, J=7.2 \mathrm{~Hz}, 3 \mathrm{H}) ;{ }^{13} \mathbf{C ~ N M R}$ 
(101 MHz, Chloroform-d) $\delta 172.1,147.3,136.1,119.1,51.9,38.5,34.2,31.8,16.1,14.2 ; \mathbf{R}_{\mathbf{f}}$ (EtOAc: $\mathrm{MeOH}=33 / 1)$ : 0.13; HR-MS (ESI) $\mathrm{m} / \mathrm{z}$ calculated for $\left[\mathrm{C}_{10} \mathrm{H}_{16} \mathrm{~N}_{2} \mathrm{O}_{2} \mathrm{SH}\right]^{+}\left([\mathrm{M}+\mathrm{H}]^{+}\right)$: 229.1005, measured: 229.1016.

Methyl 3-((1-methyl-1H-imidazol-5-yl)thio)propanoate (3d)

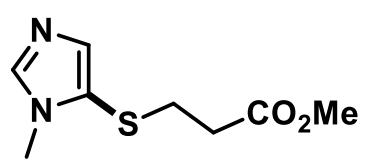

Prepared from 5-bromo-1-methyl- $1 \mathrm{H}$-imidazole $(48.3 \mathrm{mg}, 0.30 \mathrm{mmol})$ and methyl 3mercaptopropanoate $(49.9 \mu \mathrm{L}, 0.45 \mathrm{mmol})$ following general procedure A. The pure product was obtained after column chromatography (EtOAc - EtOAc: $\mathrm{MeOH}=50: 1)$ as a yellowish oil (46.8 mg, 78\%).

${ }^{1}$ H NMR (400 MHz, Chloroform-d) $\delta 7.60$ (s, 1H), $7.18(\mathrm{~s}, 1 \mathrm{H}), 3.66(\mathrm{~s}, 3 \mathrm{H}), 3.63(\mathrm{~s}, 3 \mathrm{H}), 2.80$ $(\mathrm{t}, J=7.1 \mathrm{~Hz}, 2 \mathrm{H}), 2.55$ (t, $J=7.1 \mathrm{~Hz}, 2 \mathrm{H}) ;{ }^{13} \mathbf{C ~ N M R}(101 \mathrm{MHz}$, Chloroform-d) $\delta 172.0,140.6$, 137.5, 120.8, 52.0, 34.3, 31.6, 31.1; Rf (EtOAc:MeOH = 50/1): 0.09; HR-MS (ESI) m/z calculated for $\left[\mathrm{C}_{8} \mathrm{H}_{12} \mathrm{~N}_{2} \mathrm{O}_{2} \mathrm{SH}\right]^{+}\left([\mathrm{M}+\mathrm{H}]^{+}\right): 201.0692$, measured: 201.0697.

Methyl 3-((1,4-dimethyl-1H-imidazol-2-yl)thio)propanoate (3e)<smiles>COC(=O)CCSc1nc(C)cn1C</smiles>

Prepared from 2-bromo-1,4-dimethyl- $1 \mathrm{H}$-imidazole $(52.5 \mathrm{mg}, 0.30 \mathrm{mmol})$ and methyl 3mercaptopropanoate $(49.9 \mu \mathrm{L}, 0.45 \mathrm{mmol})$ following general procedure A. The pure product was obtained after column chromatography (pentane:EtOAc $=1: 2-$ EtOAc) as a yellowish oil (48.5 mg, 75\%).

${ }^{1} \mathbf{H}$ NMR (400 MHz, Chloroform-d) $\delta 6.64(\mathrm{~d}, J=1.0 \mathrm{~Hz}, 1 \mathrm{H}), 3.65(\mathrm{~s}, 3 \mathrm{H}), 3.55(\mathrm{~s}, 3 \mathrm{H}), 3.20$ $(\mathrm{t}, J=7.0 \mathrm{~Hz}, 2 \mathrm{H}), 2.69(\mathrm{t}, J=7.0 \mathrm{~Hz}, 2 \mathrm{H}), 2.16(\mathrm{~d}, J=1.0 \mathrm{~Hz}, 3 \mathrm{H}) ;{ }^{13} \mathbf{C} \mathbf{N M R}(101 \mathrm{MHz}$, Chloroform-d) $\delta 172.2$, 139.2, 138.6, 119.4, 51.9, 34.8, 33.3, 29.7, 13.8; Rf (EtOAc): 0.32; HRMS (ESI) $\mathrm{m} / \mathrm{z}$ calculated for $\left[\mathrm{C}_{9} \mathrm{H}_{14} \mathrm{~N}_{2} \mathrm{O}_{2} \mathrm{SH}\right]^{+}\left([\mathrm{M}+\mathrm{H}]^{+}\right): 215.0849$, measured: 215.0863.

Methyl 3-((2,4-dibromo-1-methyl-1H-imidazol-5-yl)thio)propanoate (3f)<smiles>CC(=O)CCSc1c(Br)nc(Br)n1C</smiles>

Prepared from 2,4,5-tribromo-1-methyl- $1 H$-imidazole (144 mg, $0.45 \mathrm{mmol})$ and methyl 3mercaptopropanoate $(33.2 \mu \mathrm{L}, 0.30 \mathrm{mmol})$ following general procedure $\mathrm{B}$. The pure product was obtained after column chromatography (pentane:EtOAc $=4: 1)$ as a yellowish oil $(95.3 \mathrm{mg}$, $89 \%)$. 
${ }^{1}$ H NMR (400 MHz, Chloroform-d) $\delta 3.67$ (s, 3H), 3.65 (s, 3H), $2.91(\mathrm{t}, J=7.0 \mathrm{~Hz}, 2 \mathrm{H}), 2.60$ (t, $J=7.0 \mathrm{~Hz}, 2 \mathrm{H}) ;{ }^{13} \mathrm{C}$ NMR (101 MHz, Chloroform-d) $\delta 171.8,125.5,123.4,121.6,52.1$, 34.4, 33.9, 31.1; Rf (pentante:EtOAc = 2:1): 0.29; HR-MS (ESI) m/z calculated for $\left[\mathrm{C}_{8} \mathrm{H}_{10} \mathrm{~N}_{2} \mathrm{O}_{2} \mathrm{SBr}_{2} \mathrm{Na}\right]^{+}\left([\mathrm{M}+\mathrm{Na}]^{+}\right): 380.8701$, measured: 380.8703 .

2,4-Dibromo-5-((2,3-dichlorophenyl)thio)-1-methyl-1H-imidazole (3g)<smiles>Cn1c(Br)nc(Br)c1Sc1cccc(Cl)c1Cl</smiles>

Prepared from 2,4,5-tribromo-1-methyl- $1 H$-imidazole $(95.6 \mathrm{mg}, \quad 0.30 \mathrm{mmol})$ and 2,3dichlorothiophenol ( $80.6 \mathrm{mg}, 0.45 \mathrm{mmol}$ ) following general procedure A. The pure product was obtained after column chromatography (pentane:EtOAc $=6: 1)$ as a white solid $(85.7 \mathrm{mg}, 69 \%)$.

${ }^{1}$ H NMR (400 MHz, Chloroform-d) $\delta 7.29(\mathrm{dd}, J=8.0,1.4 \mathrm{~Hz}, 1 \mathrm{H}), 7.08(\mathrm{t}, J=8.0 \mathrm{~Hz}, 1 \mathrm{H})$, $6.47(\mathrm{dd}, J=8.0,1.4 \mathrm{~Hz}, 1 \mathrm{H}), 3.58(\mathrm{~s}, 3 \mathrm{H}) ;{ }^{13} \mathbf{C}$ NMR $(101 \mathrm{MHz}$, Chloroform-d) $\delta 136.7,134.2$, 129.1, 128.1 128.0, 127.7, 124.2, 123.3, 120.4, 34.0; $\mathbf{R}_{\mathbf{f}}$ (pentane:EtOAc = 4:1): 0.24; HR-MS (ESI) $\mathrm{m} / \mathrm{z}$ calculated for $\left[\mathrm{C}_{10} \mathrm{H}_{6} \mathrm{~N}_{2} \mathrm{SBr}_{2} \mathrm{Cl}_{2} \mathrm{Na}\right]^{+}\left([\mathrm{M}+\mathrm{Na}]^{+}\right)$: 438.7865 , measured: 438.7864 .

Methyl 3-((1-benzyl-2-bromo-4-chloro-1H-imidazol-5-yl)thio)propanoate (3h $\left.\mathbf{h}_{\text {major }}\right)$ and methyl 3-((1-benzyl-4,5-dichloro-1H-imidazol-2-yl)thio)propanoate $\left(\mathbf{3 h}_{\text {minor }}\right)$<smiles>CC(=O)CCSc1c(Cl)nc(Br)n1Cc1ccccc1</smiles>

$3 \mathbf{h}_{\text {major }}$ 81

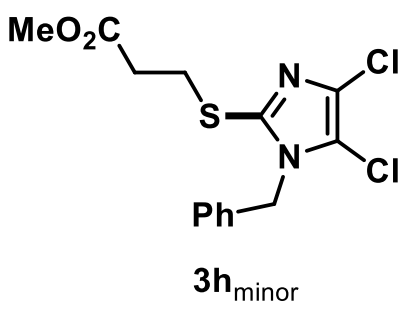

19

Prepared from 1-benzyl-2-bromo-4,5-dichloro-1H-imidazole $(137.7 \mathrm{mg}, 0.45 \mathrm{mmol})$ and methyl 3-mercaptopropanoate $(33.2 \mu \mathrm{L}, 0.30 \mathrm{mmol})$ following general procedure $\mathrm{B}$. The product was obtained after column chromatography (pentane:EtOAc $=5: 1$ ) as a yellowish oil (mixture of $\mathbf{3} \mathbf{h}_{\text {major }}$ and $\mathbf{3} \mathbf{h}_{\text {minor }}, 81: 19,83.2 \mathrm{mg}, 73 \%$ ).

${ }^{1}$ H NMR (300 MHz, Chloroform-d) $\delta 7.38-7.27$ (m, 3H), $7.15-7.07$ (m, 2H), $5.28(\mathrm{~s}, 2 \mathrm{H})$, $3.64(\mathrm{~s}, 3 \mathrm{H}), 2.61(\mathrm{t}, J=6.9 \mathrm{~Hz}, 2 \mathrm{H}), 2.47$ (t, $J=7.0 \mathrm{~Hz}, 2 \mathrm{H})$ (only major product reported); ${ }^{13}$ C NMR (75 MHz, Chloroform-d) $\delta 171.7,138.2,135.2,129.1,128.3,126.8,120.7,120.4$, 52.0, 50.1, 34.2, 31.4 (only major product reported); $\mathbf{R}_{\mathbf{f}}$ (pentane:EtOAc = 4:1): 0.19; HR-MS (ESI) $\mathrm{m} / \mathrm{z}$ calculated for $\left[\mathrm{C}_{14} \mathrm{H}_{14} \mathrm{~N}_{2} \mathrm{O}_{2} \mathrm{SBrClNa}^{+}\left([\mathrm{M}+\mathrm{Na}]^{+}\right)\right.$: 412.9519 , measured: 412.9516 (major product); $\mathrm{m} / \mathrm{z}$ calculated for $\left[\mathrm{C}_{14} \mathrm{H}_{14} \mathrm{~N}_{2} \mathrm{O}_{2} \mathrm{SCl}_{2} \mathrm{Na}\right]^{+}\left([\mathrm{M}+\mathrm{Na}]^{+}\right)$: 367.0045 , measured: 367.0042 (minor product). 
Methyl (S)-2-((tert-butoxycarbonyl)amino)-3-(5-((3-methoxy-3-oxopropyl)thio)-1-methyl$1 H$-imidazol-4-yl)propanoate (3i)<smiles>COC(=O)CCSc1c(CC(NC(C)=O)C(C)(C)C)ncn1C</smiles>

Prepared from methyl (S)-2-((tert-butoxycarbonyl)amino)-3-(5-chloro-1-methyl-1H-imidazol4-yl)propanoate $(95.3 \mathrm{mg}, \quad 0.30 \mathrm{mmol})$ and methyl 3-mercaptopropanoate $(49.9 \mu \mathrm{L}$, $0.45 \mathrm{mmol}$ ) following general procedure A. The pure product was obtained after column chromatography (EtOAc: $\mathrm{MeOH}=50: 1)$ as a yellowish oil $(119.5 \mathrm{mg}, 99 \%)$.

${ }^{1}$ H NMR (400 MHz, Chloroform-d) $\delta 7.49$ (s, 1H), $6.02(\mathrm{~d}, J=8.3 \mathrm{~Hz}, 1 \mathrm{H}), 4.59-4.45$ (m, $1 \mathrm{H}), 3.64(\mathrm{~s}, 3 \mathrm{H}), 3.61-3.58(\mathrm{~m}, 6 \mathrm{H}), 3.16(\mathrm{dd}, J=14.7,5.7 \mathrm{~Hz}, 1 \mathrm{H}), 3.01(\mathrm{dd}, J=14.6,4.9$ $\mathrm{Hz}, 1 \mathrm{H}), 2.82-2.67(\mathrm{~m}, 2 \mathrm{H}), 2.47(\mathrm{t}, J=7.0 \mathrm{~Hz}, 2 \mathrm{H}), 1.37(\mathrm{~s}, 9 \mathrm{H}) ;{ }^{13} \mathbf{C}$ NMR $(101 \mathrm{MHz}$, Chloroform-d) $\delta 172.5,171.8,155.6,144.5,139.5,118.5,79.5,53.2,52.2,51.9,34.1,31.9$, 31.3, 29.4, 28.4; Rf (EtOAc: $\mathrm{MeOH}=50: 1$ ): 0.19; HR-MS (ESI) m/z calculated for $\left[\mathrm{C}_{17} \mathrm{H}_{27} \mathrm{~N}_{3} \mathrm{O}_{6} \mathrm{SH}\right]^{+}\left([\mathrm{M}+\mathrm{H}]^{+}\right): 402.1693$, measured: 402.1698 .

Methyl (S)-2-((tert-butoxycarbonyl)amino)-3-(2-chloro-5-((3-methoxy-3-oxopropyl)thio)-1methyl-1H-imidazol-4-yl)propanoate (3j)

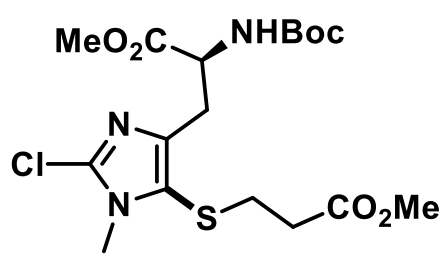

Prepared from methyl (S)-2-((tert-butoxycarbonyl)amino)-3-(2,5-dichloro-1-methyl-1Himidazol-4-yl)propanoate $(106 \mathrm{mg}, 0.30 \mathrm{mmol})$ and methyl 3-mercaptopropanoate $(49.9 \mu \mathrm{L}$, $0.45 \mathrm{mmol}$ ) following general procedure A. The pure product was obtained after column chromatography (pentane:EtOAc $=3: 2-1: 1$ ) as a yellowish oil (mixture of regioisomers: $\mathrm{C} 5: \mathrm{C} 2=91: 9,109 \mathrm{mg}, 83 \%)$.

${ }^{1}$ H NMR (400 MHz, Chloroform-d) $\delta 5.68(\mathrm{~d}, J=8.4 \mathrm{~Hz}, 1 \mathrm{H}), 4.56-4.47$ (m, 1H), 3.68 (s, $3 \mathrm{H}), 3.62(\mathrm{~s}, 3 \mathrm{H}), 3.56(\mathrm{~s}, 3 \mathrm{H}), 3.13(\mathrm{dd}, J=14.6,6.1 \mathrm{~Hz}, 1 \mathrm{H}), 3.02(\mathrm{dd}, J=14.4,4.9 \mathrm{~Hz}, 1 \mathrm{H})$, $2.79(\mathrm{td}, J=6.9,2.8 \mathrm{~Hz}, 2 \mathrm{H}), 2.50(\mathrm{t}, J=7.0 \mathrm{~Hz}, 2 \mathrm{H}), 1.39(\mathrm{~s}, 9 \mathrm{H})$ (only major isomer reported); ${ }^{13}$ C NMR (101 MHz, Chloroform-d) $\delta$ 172.3, 171.7, 155.5, 144.0, 134.3, 120.3, 79.7, 53.2, 52.3, 52.0, 34.0, 31.5, 31.4, 29.7, 28.4 (only major isomer reported); $\mathbf{R}_{\mathbf{f}}$ (pentane:EtOAc = 1:1): 0.30; HR-MS (ESI) m/z calculated for $\left[\mathrm{C}_{17} \mathrm{H}_{26} \mathrm{~N}_{3} \mathrm{O}_{6} \mathrm{SClNa}\right]^{+}\left([\mathrm{M}+\mathrm{H}]^{+}\right): 458.1123$, measured: 458.1126 . 
Methyl 3-((4-chloro-1-(2-chlorobenzyl)-1H-imidazol-5-yl)thio)propanoate (3k)<smiles>COC(=O)CCSc1c(Cl)ncn1Cc1ccccc1Cl</smiles>

Prepared from 4,5-dichloro-1-(2-chlorobenzyl)-1H-imidazole $(78.5 \mathrm{mg}, 0.30 \mathrm{mmol})$ and methyl 3-mercaptopropanoate $(49.9 \mu \mathrm{L}, 0.45 \mathrm{mmol})$ following general procedure A. The pure product was obtained after column chromatography (pentane:EtOAc $=3: 2$ ) as a colourless oil (102.3 mg, 99\%).

${ }^{1}$ H NMR (300 MHz, Chloroform-d) $\delta 7.26(\mathrm{~s}, 1 \mathrm{H}), 7.17(\mathrm{dd}, J=7.6,1.7 \mathrm{~Hz}, 1 \mathrm{H}), 7.09-6.91$ $(\mathrm{m}, 2 \mathrm{H}), 6.65(\mathrm{dd}, J=7.4,1.9 \mathrm{~Hz}, 1 \mathrm{H}), 5.04(\mathrm{~s}, 2 \mathrm{H}), 3.40(\mathrm{~s}, 3 \mathrm{H}), 2.55(\mathrm{t}, J=7.1 \mathrm{~Hz}, 2 \mathrm{H}), 2.30$ (t, $J=7.1 \mathrm{~Hz}, 2 \mathrm{H}) ;{ }^{13} \mathbf{C}$ NMR (75 MHz, Chloroform-d) $\delta 171.8,138.2,138.0,133.3,133.1$, 130.0, 129.9, 129.0, 127.6, 117.3, 52.0, 47.4, 34.4, 31.1; $\mathbf{R}_{\mathbf{f}}($ pentane:EtOAc = 1:1): 0.45; HRMS (ESI) $\mathrm{m} / \mathrm{z}$ calculated for $\left[\mathrm{C}_{14} \mathrm{H}_{14} \mathrm{Cl}_{2} \mathrm{~N}_{2} \mathrm{O}_{2} \mathrm{SNa}\right]^{+}\left([\mathrm{M}+\mathrm{Na}]^{+}\right): 367.0045$, measured: 367.0057 .

Methyl 3-((1-(4-bromobenzyl)-4-chloro-1H-imidazol-5-yl)thio)propanoate (31)<smiles>COC(=O)CCSc1c(Cl)ncn1Cc1ccc(Br)cc1</smiles>

Prepared from 1-(4-bromobenzyl)-4,5-dichloro-1H-imidazole (91.8 $\mathrm{mg}, 0.30 \mathrm{mmol}$ ) and methyl 3-mercaptopropanoate $(49.9 \mu \mathrm{L}, 0.45 \mathrm{mmol})$ following general procedure A. The pure product was obtained after column chromatography (pentane:EtOAc $=1: 1$ ) as a yellowish oil (106.8 mg, 91\%).

${ }^{1}$ H NMR (400 MHz, Chloroform-d) $\delta 7.83-7.29$ (m, 3H), 7.02 (d, J = 8.5 Hz, 2H), 5.13 (s, 2H), $3.63(\mathrm{~s}, 3 \mathrm{H}), 2.70(\mathrm{t}, J=7.0 \mathrm{~Hz}, 2 \mathrm{H}), 2.49(\mathrm{t}, J=7.0 \mathrm{~Hz}, 2 \mathrm{H}) ;{ }^{13} \mathbf{C}$ NMR $(101 \mathrm{MHz}$, Chloroform-d) $\delta 171.7,138.3,137.7,134.7,132.3,129.1,122.5,117.2,52.0,49.2,34.3,31.1$; $\mathbf{R}_{\mathbf{f}}$ (pentane:EtOAc = 1:1): 0.25; HR-MS (ESI) m/z calculated for $\left[\mathrm{C}_{14} \mathrm{H}_{14} \mathrm{~N}_{2} \mathrm{O}_{2} \mathrm{SBrClNa}\right]^{+}$ $\left([\mathrm{M}+\mathrm{Na}]^{+}\right):$412.9519, measured: 412.9524. 
Methyl 3-((4-chloro-1-(2-(6-chloronicotinamido)ethyl)-1H-imidazol-5-yl)thio)propanoate $(\mathbf{3 m})$<smiles>COC(=O)CCSc1c(Cl)ncn1CCNC(=O)c1ccc(Cl)nc1</smiles>

Prepared from 6-chloro- $N$-(2-(4,5-dichloro-1H-imidazol-1-yl)ethyl)nicotinamide (95.9 mg, $0.30 \mathrm{mmol})$ and methyl 3-mercaptopropanoate $(49.9 \mu \mathrm{L}, 0.45 \mathrm{mmol})$ following general procedure A. The pure product was obtained after column chromatography (EtOAc) as a colourless oil (106.9 mg, 88\%).

${ }^{1}$ H NMR (600 MHz, Chloroform-d) $\delta 8.74(\mathrm{dd}, J=2.5,0.8 \mathrm{~Hz}, 1 \mathrm{H}), 8.42(\mathrm{t}, J=6.2 \mathrm{~Hz}, 1 \mathrm{H})$, $8.11(\mathrm{dd}, J=8.3,2.5 \mathrm{~Hz}, 1 \mathrm{H}), 7.34(\mathrm{dd}, J=8.3,0.8 \mathrm{~Hz}, 1 \mathrm{H}), 7.25(\mathrm{~s}, 1 \mathrm{H}), 4.26-4.22(\mathrm{~m}, 2 \mathrm{H})$, $3.83-3.78(\mathrm{~m}, 2 \mathrm{H}), 3.61(\mathrm{~s}, 3 \mathrm{H}), 2.89(\mathrm{t}, J=6.8 \mathrm{~Hz}, 2 \mathrm{H}), 2.57(\mathrm{t}, J=6.8 \mathrm{~Hz}, 2 \mathrm{H}) ;{ }^{13} \mathbf{C} \mathbf{N M R}$ (151 MHz, Chloroform-d) $\delta 172.1,165.3,154.4,148.6,138.4,137.7,137.4,128.6,124.4$, 117.2, 52.2, 45.9, 40.5, 34.5, 31.2; $\mathbf{R}_{\mathbf{f}}$ (EtOAc): 0.31; HR-MS (ESI) $\mathrm{m} / \mathrm{z}$ calculated for $\left[\mathrm{C}_{15} \mathrm{H}_{16} \mathrm{~N}_{4} \mathrm{O}_{3} \mathrm{SCl}_{2} \mathrm{Na}\right]^{+}\left([\mathrm{M}+\mathrm{Na}]^{+}\right): 425.0212$, measured: 425.0195 .

Methyl 3-((3-bromothiophen-2-yl)thio)propanoate (3n)<smiles>CC(=O)CCSc1sccc1Br</smiles>

Prepared from 2,3-dibromothiophene $\quad(34.0 \mu \mathrm{L} \mathrm{mg}, \quad 0.30 \mathrm{mmol})$ and methyl 3mercaptopropanoate $(49.9 \mu \mathrm{L}, 0.45 \mathrm{mmol})$ following general procedure $\mathrm{C}$. The pure product was obtained after column chromatography (pentane:EtOAc $=15: 1)$ as a colorless oil $(54.0 \mathrm{mg}$, $64 \%)$.

${ }^{1} \mathbf{H}$ NMR (300 MHz, Chloroform-d) $\delta 7.34(\mathrm{~d}, J=5.6 \mathrm{~Hz}, 1 \mathrm{H}), 7.02(\mathrm{~d}, J=5.6 \mathrm{~Hz}, 1 \mathrm{H}), 3.68$ (s, 3H), 3.06 (t, $J=7.4 \mathrm{~Hz}, 2 \mathrm{H}), 2.63(\mathrm{t}, J=7.3 \mathrm{~Hz}, 2 \mathrm{H}) ;{ }^{13} \mathrm{C}$ NMR (75 MHz, Chloroform-d) $\delta$ 172.0, 131.2, 129.7, 129.4, 119.4, 52.0, 34.3, 32.5; $\mathbf{R}_{\mathbf{f}}$ (pentane:EtOAc = 10:1): 0.33; HR-MS (ESI) $\mathrm{m} / \mathrm{z}$ calculated for $\left[\mathrm{C}_{8} \mathrm{H}_{9} \mathrm{O}_{2} \mathrm{~S}_{2} \mathrm{BrNa}\right]^{+}\left([\mathrm{M}+\mathrm{Na}]^{+}\right)$: 304.9099, measured: 304.9094. 
Methyl 3-((5-bromothiophen-2-yl)thio)propanoate (3o)<smiles>CC(=O)CCSc1ccc(Br)s1</smiles>

Prepared from 2,5-dibromothiophene $(34.0 \mu \mathrm{L} \mathrm{mg}, \quad 0.30 \mathrm{mmol})$ and methyl 3mercaptopropanoate $(49.9 \mu \mathrm{L}, 0.45 \mathrm{mmol})$ following general procedure $\mathrm{C}$. The product was obtained after column chromatography (pentane:EtOAc $=15: 1$ ) as an inseparable mixture with methyl 3-(thiophen-2-ylthio)propanoate (via debromination, $10 \mathrm{~mol} \%$ with respect to the desired product) appearing as a colorless oil $(37.2 \mathrm{mg}, 40 \%$, yield was corrected by subtracting the respective amount of byproduct).

${ }^{1}$ H NMR (400 MHz, Chloroform-d) $\delta 6.97-6.90(\mathrm{~m}, 2 \mathrm{H}), 3.69(\mathrm{~s}, 3 \mathrm{H}), 2.98(\mathrm{t}, J=7.3 \mathrm{~Hz}$, 2H), 2.62 (t, $J=7.2 \mathrm{~Hz}, 2 \mathrm{H}) ;{ }^{13} \mathbf{C}$ NMR (101 MHz, Chloroform-d) $\delta 172.0,135.7,134.5,130.7$, 115.1, 52.0, 34.4, 33.9; $\mathbf{R}_{\mathbf{f}}$ (pentane:EtOAc = 15:1): 0.24; HR-MS (ESI) m/z calculated for $\left[\mathrm{C}_{8} \mathrm{H}_{9} \mathrm{O}_{2} \mathrm{~S}_{2} \mathrm{BrNa}\right]^{+}\left([\mathrm{M}+\mathrm{Na}]^{+}\right): 304.9099$, measured: 304.9109 .

Methyl 3-((5-phenylfuran-2-yl)thio)propanoate (3p)

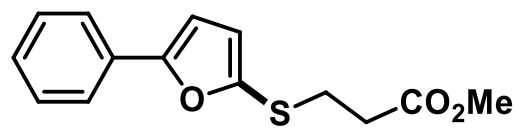

Prepared from 2-bromo-5-phenylfuran $\quad(66.9 \mathrm{mg}, \quad 0.30 \mathrm{mmol})$ and methyl 3mercaptopropanoate $(49.9 \mu \mathrm{L}, 0.45 \mathrm{mmol})$ following general procedure A. The pure product was obtained after column chromatography (pentane:EtOAc $=15: 1$ ) as a yellowish oil (31.3 mg, 40\%).

${ }^{1}$ H NMR (400 MHz, Chloroform-d) $\delta 7.70-7.64(\mathrm{~m}, 2 \mathrm{H}), 7.43-7.36(\mathrm{~m}, 2 \mathrm{H}), 7.32-7.24$ $(\mathrm{m}, 1 \mathrm{H}), 6.62(\mathrm{q}, J=3.3 \mathrm{~Hz}, 2 \mathrm{H}), 3.67(\mathrm{~s}, 3 \mathrm{H}), 3.05(\mathrm{t}, J=7.2 \mathrm{~Hz}, 2 \mathrm{H}), 2.69(\mathrm{t}, J=7.2 \mathrm{~Hz}, 2 \mathrm{H})$; ${ }^{13}$ C NMR (101 MHz, Chloroform-d) $\delta 172.1,157.2,144.2,130.4,128.9,128.0,124.0,120.1$, 106.8, 52.0, 35.0, 31.0; $\mathbf{R}_{\mathbf{f}}$ (pentane:EtOAc = 10:1): 0.32; HR-MS (ESI) $\mathrm{m} / \mathrm{z}$ calculated for $\left[\mathrm{C}_{14} \mathrm{H}_{14} \mathrm{O}_{3} \mathrm{SNa}\right]^{+}\left([\mathrm{M}+\mathrm{Na}]^{+}\right): 285.0556$, measured: 285.0575 .

Methyl 3-((4-bromo-5-phenylfuran-2-yl)thio)propanoate (3q)

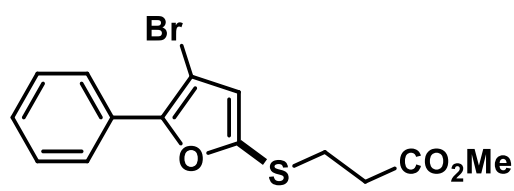

Prepared from 3,5-dibromo-2-phenylfuran (54.1 mg, $0.18 \mathrm{mmol})$, methyl 3mercaptopropanoate $(29.9 \mu \mathrm{L}, \quad 0.27 \mathrm{mmol})$ and $\quad\left[\operatorname{Ir}\left(\mathrm{dFCF}_{3} p p y\right)_{2}(\mathrm{dtbbpy}) \mathrm{PF}_{6} \quad(4.0 \mathrm{mg}\right.$, $0.0036 \mathrm{mmol}, 2 \mathrm{~mol} \%$ ) following general procedure A. The pure product was obtained after column chromatography (pentane:EtOAc $=20: 1)$ as a yellowish oil $(43.8 \mathrm{mg}, 71 \%)$.

${ }^{1} \mathbf{H}$ NMR (300 MHz, Chloroform-d) $\delta 7.99-7.92(\mathrm{~m}, 2 \mathrm{H}), 7.47-7.40$ (m, 2H), $7.38-7.31$ $(\mathrm{m}, 1 \mathrm{H}), 6.65(\mathrm{~s}, 1 \mathrm{H}), 3.68(\mathrm{~s}, 3 \mathrm{H}), 3.06(\mathrm{t}, J=7.2 \mathrm{~Hz}, 2 \mathrm{H}), 2.69(\mathrm{t}, J=7.2 \mathrm{~Hz}, 2 \mathrm{H}) ;{ }^{13} \mathbf{C ~ N M R}$ (75 MHz, Chloroform-d) $\delta 171.9,152.2,144.7,129.4,128.7,128.6,125.8,123.1,96.6,52.0$, 
34.9, 30.8; $\mathbf{R}_{\mathbf{f}}$ (pentane:EtOAc = 15:1): 0.27; HR-MS (ESI) $\mathrm{m} / \mathrm{z}$ calculated for $\left[\mathrm{C}_{14} \mathrm{H}_{13} \mathrm{BrO}_{3} \mathrm{SNa}\right]^{+}\left([\mathrm{M}+\mathrm{Na}]^{+}\right): 364.9641$, measured: 364.9644 .

Methyl 3-((1-methyl-5-phenyl-1H-pyrrol-2-yl)thio)propanoate (3r)<smiles>COC(=O)CCSc1ccc(-c2ccccc2)n1C</smiles>

Prepared from 2-chloro-1-methyl-5-phenyl-1 $\mathrm{H}$-pyrrole $(57.5 \mathrm{mg}, 0.30 \mathrm{mmol})$ and methyl 3mercaptopropanoate $(99.7 \mu \mathrm{L}, 0.90 \mathrm{mmol}, 3.0$ equiv) following general procedure $\mathrm{A}$. The pure product was obtained after column chromatography (pentane:EtOAc $=9: 1$ ) as an orange oil (39.1 mg, 47\%).

${ }^{1} \mathbf{H}$ NMR (400 MHz, Chloroform-d) $\delta 7.43-7.38(\mathrm{~m}, 4 \mathrm{H}), 7.36-7.31(\mathrm{~m}, 1 \mathrm{H}), 6.48(\mathrm{~d}, J=$ $3.7 \mathrm{~Hz}, 1 \mathrm{H}), 6.21(\mathrm{~d}, J=3.7 \mathrm{~Hz}, 1 \mathrm{H}), 3.70(\mathrm{~s}, 3 \mathrm{H}), 3.66(\mathrm{~s}, 3 \mathrm{H}), 2.87$ (t, $J=7.2 \mathrm{~Hz}, 2 \mathrm{H}), 2.64$ (t, $J=7.2 \mathrm{~Hz}, 2 \mathrm{H}) ;{ }^{13} \mathbf{C}$ NMR (101 MHz, Chloroform-d) $\delta 172.4,138.3,133.6,128.8,128.6$, 127.4, 121.0, 118.6, 108.8, 51.9, 34.4, 32.4, 31.7; $\mathbf{R}_{\mathbf{f}}$ (pentane:EtOAc = 9:1): 0.36; HR-MS (ESI) $\mathrm{m} / \mathrm{z}$ calculated for $\left[\mathrm{C}_{15} \mathrm{H}_{17} \mathrm{NO}_{2} \mathrm{SNa}\right]^{+}\left([\mathrm{M}+\mathrm{Na}]^{+}\right)$: 298.0872, measured: 298.0874.

Methyl 3-((3,4-dichloro-1-methyl-5-phenyl-1H-pyrrol-2-yl)thio)propanoate (3s)<smiles>COC(=O)CCSc1c(Cl)c(Cl)c(-c2ccccc2)n1C</smiles>

Prepared from 2,3,4-trichloro-1-methyl-5-phenyl-1 $H$-pyrrole (78.2 mg, $0.30 \mathrm{mmol}$ ) and methyl 3 -mercaptopropanoate $(99.7 \mu \mathrm{L}, 0.90 \mathrm{mmol}, 3.0$ equiv) following general procedure $\mathrm{A}$. The pure product was obtained after column chromatography (pentane:EtOAc $=9: 1$ ) as an orange oil (54.7 mg, 53\%).

${ }^{1}$ H NMR (400 MHz, Chloroform-d) $\delta 7.50-7.45(\mathrm{~m}, 2 \mathrm{H}), 7.44-7.40(\mathrm{~m}, 1 \mathrm{H}), 7.40-7.37$ $(\mathrm{m}, 2 \mathrm{H}), 3.65(\mathrm{~s}, 3 \mathrm{H}), 3.59(\mathrm{~s}, 3 \mathrm{H}), 2.96(\mathrm{t}, J=7.1 \mathrm{~Hz}, 2 \mathrm{H}), 2.65(\mathrm{t}, J=7.0 \mathrm{~Hz}, 2 \mathrm{H}) ;{ }^{13} \mathbf{C ~ N M R}$ (101 MHz, Chloroform-d) $\delta 172.1,133.2,130.3,129.9,128.8,128.7,119.3,117.7,109.3,51.9$, 34.7, 33.5, 31.2; $\mathbf{R}_{\mathbf{f}}$ (pentane:EtOAc = 9:1): 0.25; HR-MS (ESI) $\mathrm{m} / \mathrm{z}$ calculated for $\left[\mathrm{C}_{15} \mathrm{H}_{15} \mathrm{NO}_{2} \mathrm{SCl}_{2} \mathrm{Na}\right]^{+}\left([\mathrm{M}+\mathrm{Na}]^{+}\right): 366.0093$, measured: 366.0095 .

Methyl 3-((3,4-dibromo-1-methyl-5-phenyl-1H-pyrrol-2-yl)thio)propanoate (3t)<smiles>COC(=O)CCSc1c(Br)c(Br)c(-c2ccccc2)n1C</smiles>

Prepared from 2,3,4-tribromo-1-methyl-5-phenyl-1H-pyrrole (118 mg, $0.30 \mathrm{mmol})$ and methyl 3-mercaptopropanoate $(49.8 \mu \mathrm{L}, 0.45 \mathrm{mmol})$ following general procedure $\mathrm{A}$. The pure product 
was obtained after column chromatography (pentane:EtOAc $=9: 1)$ as an orange oil $(117 \mathrm{mg}$, $90 \%)$.

${ }^{1} \mathbf{H}$ NMR (400 MHz, Chloroform-d) $\delta 7.49-7.44(\mathrm{~m}, 2 \mathrm{H}), 7.43-7.39$ (m, 1H), $7.38-7.35$ $(\mathrm{m}, 2 \mathrm{H}), 3.64(\mathrm{~s}, 3 \mathrm{H}), 3.60(\mathrm{~s}, 3 \mathrm{H}), 2.97(\mathrm{t}, J=7.0 \mathrm{~Hz}, 2 \mathrm{H}), 2.65(\mathrm{t}, J=7.0 \mathrm{~Hz}, 2 \mathrm{H}) ;{ }^{13} \mathbf{C ~ N M R}$ (101 MHz, Chloroform-d) $\delta 172.0,135.7,130.8,130.4,128.8,128.6,120.4,109.9,98.6,51.9$, 34.6, 34.3, 31.3; Rf (pentane:EtOAc = 9:1): 0.26; HR-MS (ESI) $\mathrm{m} / \mathrm{z}$ calculated for $\left[\mathrm{C}_{15} \mathrm{H}_{15} \mathrm{NO}_{2} \mathrm{SBr}_{2} \mathrm{Na}\right]^{+}\left([\mathrm{M}+\mathrm{Na}]^{+}\right)$: 453.9082, measured: 453.9078 .

Dimethyl 3,3'-((1-methyl-1H-pyrrole-2,5-diyl)bis(sulfanediyl))dipropionate (3u)

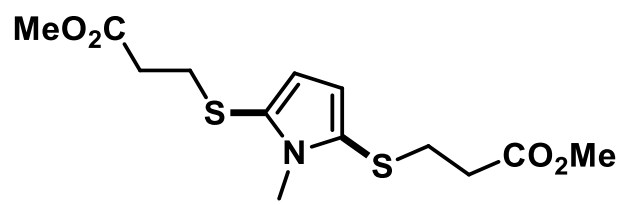

Prepared from 2,5-dichloro-1-methyl- $1 H$-pyrrole $^{48}(45 \mathrm{mg}, 0.30 \mathrm{mmol})$ and methyl 3mercaptopropanoate $(100 \mu \mathrm{L}, 0.90 \mathrm{mmol}, 3.0$ equiv) following general procedure A. The product was obtained after column chromatography (pentane:EtOAc $=5: 1$ ) as an inseparable mixture with dimethyl 3,3'-disulfanediyldipropionate (via dimerization of thiyl radicals, $10 \mathrm{~mol} \%$ with respect to the desired product) appearing as a white solid (64.4 mg, 63\%, yield was corrected by subtracting the respective amount of byproduct).

${ }^{1} \mathbf{H}$ NMR (300 MHz, Chloroform-d) $\delta 6.35$ (s, 2H), 3.73 (s, 3H), 3.64 (s, 6H), 2.83 (t, J=6.9 $\mathrm{Hz}, 4 \mathrm{H}), 2.55$ (t, $J=7.3 \mathrm{~Hz}, 4 \mathrm{H}) ;{ }^{13} \mathbf{C}$ NMR (75 MHz, Chloroform-d) $\delta 172.2,123.4,118.0$, 51.9, 34.3, 31.5, 31.4; $\mathbf{R}_{\mathbf{f}}$ (pentane:EtOAc = 5:1): 0.16; HR-MS (ESI) m/z calculated for $\left[\mathrm{C}_{13} \mathrm{H}_{19} \mathrm{NO}_{4} \mathrm{~S}_{2} \mathrm{Na}\right]^{+}\left([\mathrm{M}+\mathrm{Na}]^{+}\right): 340.0648$, measured: 340.0654 .

Methyl 3-((2-aminothiazol-5-yl)thio)propanoate (3v)<smiles>COC(C)=O</smiles>

Prepared from 5-bromothiazol-2-amine (54 mg, $0.30 \mathrm{mmol}$ ) and methyl 3-mercaptopropanoate $(49.9 \mu \mathrm{L}, 0.45 \mathrm{mmol})$ following general procedure A. The pure product was obtained after column chromatography (pentane:EtOAc $=1: 2$ ) as a yellowish solid $(42.2 \mathrm{mg}, 64 \%)$.

${ }^{1} \mathbf{H}$ NMR (400 MHz, Chloroform-d) $\delta 7.08(\mathrm{~s}, 1 \mathrm{H}), 5.31$ (s, 2H), $3.68(\mathrm{~s}, 3 \mathrm{H}), 2.88$ (t, J = 7.2 $\mathrm{Hz}, 2 \mathrm{H}), 2.62(\mathrm{t}, J=7.2 \mathrm{~Hz}, 2 \mathrm{H}) ;{ }^{13} \mathbf{C}$ NMR (101 MHz, Chloroform-d) $\delta 172.2,171.4,146.8$, 116.5, 52.0, 34.3, 33.2; Rf (EtOAc): 0.30; HR-MS (ESI) m/z calculated for $\left[\mathrm{C}_{7} \mathrm{H}_{10} \mathrm{~N}_{2} \mathrm{O}_{2} \mathrm{~S}_{2} \mathrm{H}\right]^{+}$ $\left([\mathrm{M}+\mathrm{H}]^{+}\right):$219.0256, measured: 219.0276 . 
4-Chloro-5-(dodecylthio)-1-methyl-1H-imidazole (3w)

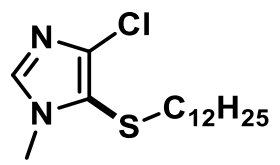

Prepared from 4,5-dichloro-1-methyl- $1 H$-imidazole $(45.3 \mathrm{mg}, 0.30 \mathrm{mmol})$ and dodecane-1thiol $(108 \mu \mathrm{L}, 0.45 \mathrm{mmol})$ following general procedure $\mathrm{A}$. The pure product was obtained after column chromatography (pentane:EtOAc $=3: 2)$ as a yellowish oil $(94.5 \mathrm{mg}, 99 \%)$.

${ }^{1}$ H NMR (300 MHz, Chloroform-d) $\delta 7.43(\mathrm{~s}, 1 \mathrm{H}), 3.63(\mathrm{~s}, 3 \mathrm{H}), 2.60(\mathrm{t}, J=7.3 \mathrm{~Hz}, 2 \mathrm{H}), 1.56$ - 1.39 (m, 2H), 1.22 (s, 18H), 0.85 (t, $J=6.5 \mathrm{~Hz}, 3 \mathrm{H}) ;{ }^{13} \mathbf{C ~ N M R}(75 \mathrm{MHz}$, Chloroform-d) $\delta$ 137.4, 137.0, 118.5, 36.3, 32.9, 32.0, 29.75, 29.68, 29.63, 29.55, 29.4, 29.2, 28.5, 22.8, 14.2 (one signal overlapping); Rf (pentane:EtOAc = 1:1): 0.45; HR-MS (ESI) $\mathrm{m} / \mathrm{z}$ calculated for $\left[\mathrm{C}_{16} \mathrm{H}_{29} \mathrm{~N}_{2} \mathrm{SClNa}\right]^{+}\left([\mathrm{M}+\mathrm{Na}]^{+}\right): 339.1632$, measured: 339.1642 .

4-Chloro-5-(cyclohexylthio)-1-methyl-1H-imidazole (3x)<smiles>Cn1cnc(Cl)c1SC1CCCCC1</smiles>

Prepared from 4,5-dichloro-1-methyl-1H-imidazole $\quad(45.3 \mathrm{mg}, \quad 0.30 \mathrm{mmol})$ and cyclohexanethiol $(110 \mu \mathrm{L}, 0.90 \mathrm{mmol}, 3.0$ equiv) following general procedure A. The pure product was obtained after column chromatography (pentane:EtOAc $=1: 1$ ) as an orange oil (68.8 mg, 99\%).

${ }^{1} \mathbf{H}$ NMR (400 MHz, Chloroform-d) $\delta 7.45$ (s, 1H), $3.62(\mathrm{~s}, 3 \mathrm{H}), 2.83(\mathrm{tt}, J=10.8,3.7 \mathrm{~Hz}, 1 \mathrm{H})$, $1.88-1.80(\mathrm{~m}, 2 \mathrm{H}), 1.77-1.69(\mathrm{~m}, 2 \mathrm{H}), 1.59-1.52(\mathrm{~m}, 1 \mathrm{H}), 1.33-1.16(\mathrm{~m}, 5 \mathrm{H}) ;{ }^{13} \mathbf{C}$ NMR (101 MHz, Chloroform-d) $\delta$ 137.7, 137.6, 117.8, 48.8, 33.5, 33.0, 26.0, 25.5; $\mathbf{R}_{\mathbf{f}}$ (pentane:EtOAc = 1:1): 0.39; HR-MS (ESI) $\mathrm{m} / \mathrm{z}$ calculated for $\left[\mathrm{C}_{10} \mathrm{H}_{15} \mathrm{~N}_{2} \mathrm{SClNa}\right]^{+}\left([\mathrm{M}+\mathrm{Na}]^{+}\right)$: 253.0537, measured: 253.0549 .

5-(Benzylthio)-4-chloro-1-methyl-1H-imidazole (3y)<smiles>Cn1cnc(Cl)c1SCc1ccccc1</smiles>

Prepared from 4,5-dichloro-1-methyl- $1 H$-imidazole $(45.3 \mathrm{mg}, 0.30 \mathrm{mmol})$ and benzyl mercaptan $(53 \mu \mathrm{L}, 0.45 \mathrm{mmol})$ following general procedure A. The pure product was obtained after column chromatography (toluene:EtOAc $=3: 1$ ) as a yellowish oil $(42.9 \mathrm{mg}, 60 \%)$.

${ }^{1}$ H NMR (300 MHz, Chloroform-d) $\delta 7.30(\mathrm{~s}, 1 \mathrm{H}), 7.24-7.16(\mathrm{~m}, 3 \mathrm{H}), 7.04-6.94(\mathrm{~m}, 2 \mathrm{H})$, 3.79 (s, 2H), 2.97 (s, 3H); ${ }^{13}$ C NMR (75 MHz, Chloroform-d) $\delta 137.84,137.78,137.6,128.9$, 128.7, 127.6, 117.5, 40.4, 32.3; Rf (toluene:EtOAc = 2:1): 0.28; HR-MS (ESI) m/z calculated for $\left[\mathrm{C}_{11} \mathrm{H}_{11} \mathrm{~N}_{2} \mathrm{SClNa}\right]^{+}\left([\mathrm{M}+\mathrm{Na}]^{+}\right): 261.0224$, measured: 261.0229 . 
2-((4-Chloro-1-methyl-1H-imidazol-5-yl)thio)ethan-1-ol (3z)<smiles>Cn1cnc(Cl)c1SCCO</smiles>

Prepared from 4,5-dichloro-1-methyl- $1 \mathrm{H}$-imidazole $\quad(45.3 \mathrm{mg}, \quad 0.30 \mathrm{mmol})$ and 2mercaptoethanol ( $32 \mu \mathrm{L}, 0.45 \mathrm{mmol}$ ) following general procedure A. No aqueous work-up was performed due to water solubility of the product. Instead, $\mathrm{Et}_{3} \mathrm{~N}(0.12 \mathrm{~mL}, 0.90 \mathrm{mmol}, 3.0$ equiv) was added to the crude reaction mixture and DMA was removed by co-evaporation with toluene (5 times). The pure product was obtained after column chromatography (EtOAc: $\mathrm{MeOH}=50: 1$ ) as a white solid (31.2 $\mathrm{mg}, 54 \%)$.

${ }^{1}$ H NMR (400 MHz, Chloroform-d) $\delta 7.50$ (s, 1H), 3.69 (s, 3H), 3.67 (t, $\left.J=6.0 \mathrm{~Hz}, 2 \mathrm{H}\right), 2.88$ (s, 1H), 2.79 (t, $J=6.0 \mathrm{~Hz}, 2 \mathrm{H}) ;{ }^{13} \mathbf{C}$ NMR (101 MHz, Chloroform-d) $\delta$ 137.8, 137.3, 117.6, 60.6, 39.0, 33.1; Rf (EtOAc: $\mathrm{MeOH}=33: 1$ ): 0.13; HR-MS (ESI) m/z calculated for $\left[\mathrm{C}_{6} \mathrm{H}_{9} \mathrm{ClN}_{2} \mathrm{OSNa}\right]^{+}\left([\mathrm{M}+\mathrm{Na}]^{+}\right): 215.0016$, measured: 215.0033 .

4-Chloro-5-((3-chloropropyl)thio)-1-methyl-1H-imidazole (3aa)<smiles>Cn1cnc(Cl)c1SCCCCl</smiles>

Prepared from 4,5-dichloro-1-methyl- $1 \mathrm{H}$-imidazole $\quad(45.3 \mathrm{mg}, \quad 0.30 \mathrm{mmol}) \quad$ and 3 chloropropane-1-thiol (49.8 $\mathrm{mg}, 0.45 \mathrm{mmol}$ ) following general procedure A. The pure product was obtained after column chromatography (pentane:EtOAc $=1: 1)$ as a yellowish oil $(65.7 \mathrm{mg}$, $97 \%)$.

${ }^{1}$ H NMR (400 MHz, Chloroform-d) $\delta 7.47$ (s, 1H), $3.68(\mathrm{t}, J=6.1 \mathrm{~Hz}, 2 \mathrm{H}), 3.66(\mathrm{~s}, 3 \mathrm{H}), 2.76$ (t, $J=7.0 \mathrm{~Hz}, 2 \mathrm{H}), 1.95$ (ddd, $J=12.9,7.0,6.0 \mathrm{~Hz}, 2 \mathrm{H}) ;{ }^{13} \mathbf{C}$ NMR (101 MHz, Chloroform-d) $\delta 137.9,137.6,117.5,43.0,33.1,32.9,32.0 ; \mathbf{R}_{\mathbf{f}}$ (pentane:EtOAc = 1:1): 0.17; HR-MS (ESI) $\mathrm{m} / \mathrm{z}$ calculated for $\left[\mathrm{C}_{7} \mathrm{H}_{10} \mathrm{~N}_{2} \mathrm{SCl}_{2} \mathrm{Na}\right]^{+}\left([\mathrm{M}+\mathrm{Na}]^{+}\right)$: 246.9834, measured: 246.9839 .

1,3-Bis((4-chloro-1-methyl-1H-imidazol-5-yl)thio)propane (3ab)<smiles>Cn1cnc(Cl)c1SCCCSc1c(Cl)ncn1C</smiles>

Prepared from 4,5-dichloro-1-methyl-1H-imidazole (113 mg, $0.75 \mathrm{mmol}, 2.5$ equiv) and propane-1,3-dithiol $(30.1 \mu \mathrm{L}, 0.30 \mathrm{mmol}, 1.0$ equiv) following general procedure $\mathrm{B}$ using $\mathrm{K}_{2} \mathrm{HPO}_{4}$ (105 mg, $0.6 \mathrm{mmol}, 2.0$ equiv). The pure product was obtained after column chromatography (DCM:MeOH $=19: 1)$ as a yellow oil $(93.9 \mathrm{mg}, 93 \%)$.

${ }^{1} \mathbf{H}$ NMR (300 MHz, Chloroform-d) $\delta 7.45$ (s, 2H), 3.62 (s, 6H), $2.72(\mathrm{t}, J=7.0 \mathrm{~Hz}, 4 \mathrm{H}), 1.69$ $(\mathrm{p}, J=7.0 \mathrm{~Hz}, 2 \mathrm{H}) ;{ }^{13} \mathbf{C}$ NMR $(75 \mathrm{MHz}$, Chloroform-d) $\delta 137.9,137.3,117.5,34.4,32.9,29.2$; 
Rf $(\mathrm{DCM}: \mathrm{MeOH}=19: 1): 0.18$; HR-MS (ESI) $\mathrm{m} / \mathrm{z}$ calculated for $\left[\mathrm{C}_{11} \mathrm{H}_{14} \mathrm{~N}_{4} \mathrm{~S}_{2} \mathrm{Cl}_{2} \mathrm{Na}\right]^{+}$ $\left([\mathrm{M}+\mathrm{Na}]^{+}\right): 358.9919$, measured: 358.9929 .

Methyl $N$-(tert-butoxycarbonyl)-S-(4-chloro-1-methyl-1H-imidazol-5-yl)- $L$-cysteinate (3ac)<smiles>CC(=O)OCC(NC(=O)OCc1ccccc1)c1c(Cl)ncn1C</smiles>

Prepared from 4,5-dichloro-1-methyl- $1 H$-imidazole $(45.3 \mathrm{mg}, 0.30 \mathrm{mmol})$, methyl (tertbutoxycarbonyl)- $L$-cysteinate $\left(212 \mathrm{mg}, 0.90 \mathrm{mmol}, 3.0\right.$ equiv) and $\left[\operatorname{Ir}\left(\mathrm{dFCF}_{3} \text { ppy }\right)_{2}(\mathrm{dtbbpy})\right] \mathrm{PF}_{6}$ (13.4 mg, $4 \mathrm{~mol} \%$ ) following general procedure A. The pure product was obtained after column chromatography (pentane:EtOAc $=1: 1-1: 2)$ as a colorless oil $(91.6 \mathrm{mg}, 87 \%)$.

${ }^{1}$ H NMR (300 MHz, Chloroform-d) $\delta 7.47(\mathrm{~s}, 1 \mathrm{H}), 5.45$ (d, J = 7.8 Hz, 1H), 4.54 - 4.32 (m, $1 \mathrm{H}), 3.63(\mathrm{~s}, 3 \mathrm{H}), 3.56(\mathrm{~s}, 3 \mathrm{H}), 3.24(\mathrm{dd}, J=13.9,4.7 \mathrm{~Hz}, 1 \mathrm{H}), 3.09(\mathrm{dd}, J=13.9,4.8 \mathrm{~Hz}, 1 \mathrm{H})$, 1.39 (s, 9H); ${ }^{13}$ C NMR (101 MHz, Chloroform-d) $\delta$ 170.6, 155.1, 137.8, 136.7, 116.7, 80.4, 53.0, 52.8, 37.2, 33.0, 28.3; $\mathbf{R}_{\mathbf{f}}$ (EtOAc): 0.27; HR-MS (ESI) $\mathrm{m} / \mathrm{z}$ calculated for $\left[\mathrm{C}_{13} \mathrm{H}_{20} \mathrm{~N}_{3} \mathrm{O}_{4} \mathrm{SClNa}\right]^{+}\left([\mathrm{M}+\mathrm{Na}]^{+}\right): 372.0755$, measured: 372.0764 ; HPLC $>99 \%$ ee (Chiralpak OD-H, hexane: $i$-PrOH $=80: 20$, flow rate: $1 \mathrm{~mL} / \mathrm{min}$, detector: $254 \mathrm{~nm}, t_{\mathrm{R}}$ (minor) $=10.3 \mathrm{~min}$, $t_{\mathrm{R}}($ major $)=14.3 \mathrm{~min}$.

\section{HPLC traces}

Racemic compound:

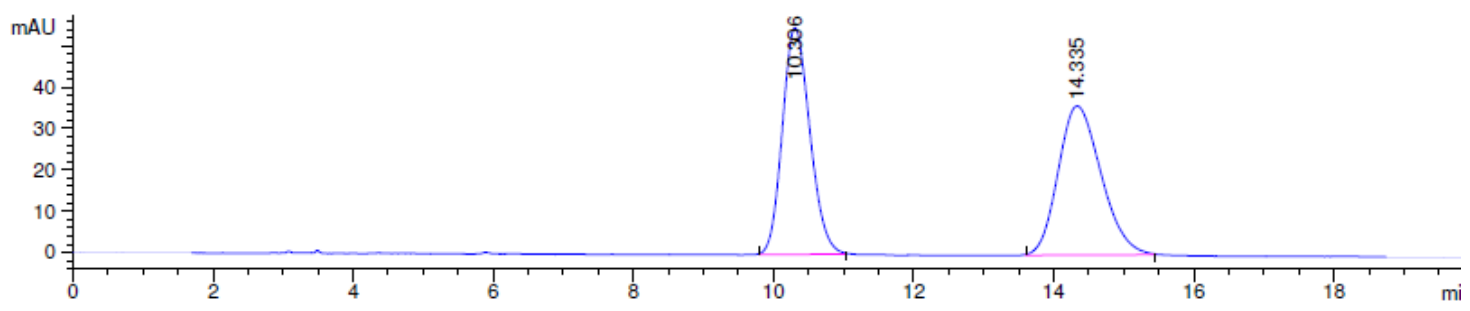

Signal 2: DAD1 B, Sig=254,16 Ref=360,100

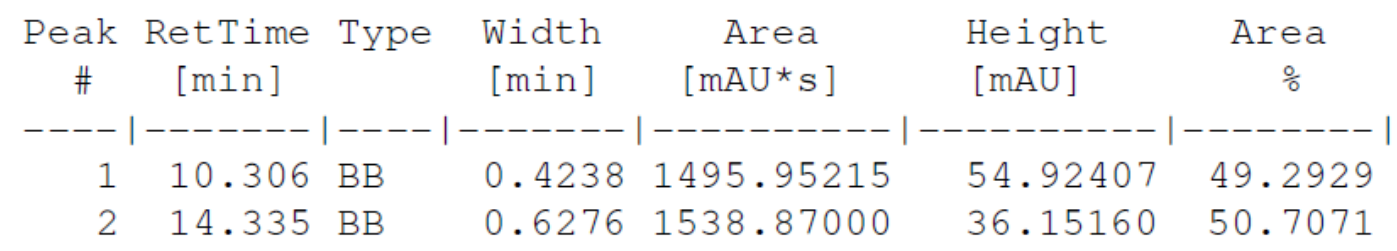

Totals : $\quad 3034.82214 \quad 91.07566$ 
Enantioenriched compound:

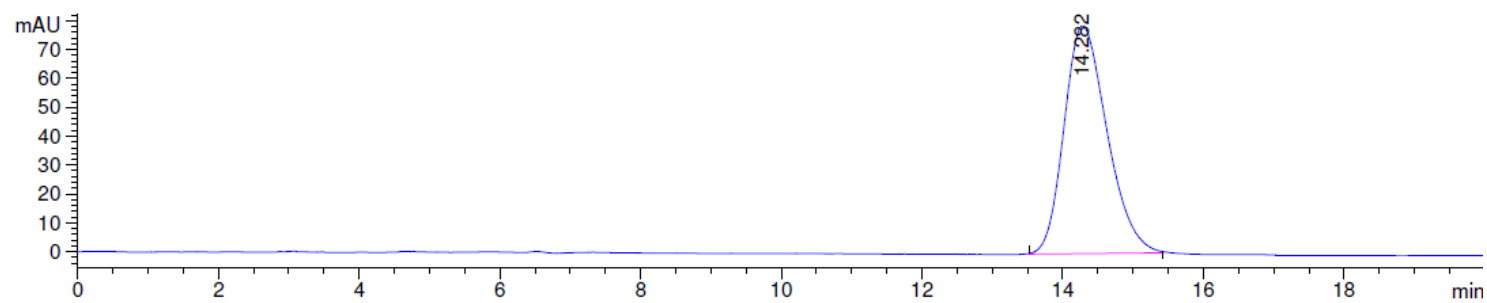

Signal 2: DAD1 B, Sig=254,16 Ref=360,100

\begin{tabular}{|c|c|c|c|c|c|c|}
\hline $\begin{array}{c}\text { Peak } \\
\quad \#\end{array}$ & $\begin{array}{c}\text { RetTime } \\
\text { [min] }\end{array}$ & Type & $\begin{array}{l}\text { Width } \\
\text { [min] }\end{array}$ & $\begin{array}{c}\text { Area } \\
{\left[\mathrm{mAU}^{*} \mathrm{~s}\right]}\end{array}$ & $\begin{array}{l}\text { Height } \\
\text { [mAU] }\end{array}$ & $\begin{array}{c}\text { Area } \\
\%\end{array}$ \\
\hline 1 & 14.282 & $\mathrm{BB}$ & 0.6352 & 3342.60986 & 78.90364 & 100.0000 \\
\hline Tota] & Ls : & & & 3342.60986 & 78.90364 & \\
\hline
\end{tabular}

4-Chloro-1-methyl-5-((4-(trifluoromethyl)phenyl)thio)-1H-imidazole (3ad)<smiles>Cn1cnc(Cl)c1Sc1ccc(C(F)(F)F)cc1</smiles>

Prepared from 4,5-dichloro-1-methyl- $1 H$-imidazole $\quad(45.3 \mathrm{mg}, \quad 0.30 \mathrm{mmol})$ and 4(trifluoromethyl)thiophenol $(62 \mu \mathrm{L}, 0.45 \mathrm{mmol})$ following general procedure A. The pure product was obtained after column chromatography (pentane:EtOAc $=3: 2-1: 1$ ) as a white solid (86.5 mg, 99\%).

${ }^{1}$ H NMR (300 MHz, Chloroform-d) $\delta 7.64$ (s, 1H), 7.48 (d, $\left.J=8.2 \mathrm{~Hz}, 2 \mathrm{H}\right), 7.09$ (d, $J=8.1$ $\mathrm{Hz}, 2 \mathrm{H}), 3.58$ (s, 3H); ${ }^{13} \mathrm{C}$ NMR (75 MHz, Chloroform-d) $\delta 140.5,139.6,139.1,128.6$ (q, J = $33.4 \mathrm{~Hz}), 126.3$ (q, J = 3.8 Hz), 126.0, 122.2, 114.4, 33.0; ${ }^{19}$ F NMR (282 MHz, Chloroform-d) $\delta$-62.5; Rf (pentane:EtOAc = 1:1): 0.31; HR-MS (ESI) $\mathrm{m} / \mathrm{z}$ calculated for $\left[\mathrm{C}_{11} \mathrm{H}_{8} \mathrm{ClF}_{3} \mathrm{~N}_{2} \mathrm{SNa}^{+}\right.$ $\left([\mathrm{M}+\mathrm{Na}]^{+}\right): 314.9941$, measured: 314.9947 .

4-Chloro-5-((2,3-dichlorophenyl)thio)-1-methyl-1H-imidazole (3ae)<smiles>Cn1cnc(Cl)c1Sc1cccc(Cl)c1Cl</smiles>

Prepared from 4,5-dichloro-1-methyl-1H-imidazole $\quad(45.3 \mathrm{mg}, \quad 0.30 \mathrm{mmol})$ and 2,3dichlorothiophenol ( $80.6 \mathrm{mg}, 0.45 \mathrm{mmol}$ ) following general procedure A. The pure product was obtained after column chromatography (pentane:EtOAc $=1: 1-1: 2)$ as a white solid $(86.0 \mathrm{mg}$, $98 \%)$. 
${ }^{1} \mathbf{H}$ NMR (400 MHz, Chloroform-d) $\delta 7.68(\mathrm{~s}, 1 \mathrm{H}), 7.27(\mathrm{dd}, J=8.0,1.3 \mathrm{~Hz}, 1 \mathrm{H}), 7.05(\mathrm{t}, J=$ $8.0 \mathrm{~Hz}, 1 \mathrm{H}), 6.42(\mathrm{dd}, J=8.1,1.4 \mathrm{~Hz}, 1 \mathrm{H}), 3.60$ (s, 3H); ${ }^{13} \mathrm{C}$ NMR (101 MHz, Chloroform-d) $\delta 139.9,139.4,137.4,134.0,128.8,127.9,127.8,124.0,114.0,33.0$; Rf (EtOAc): 0.31; HRMS (ESI) $\mathrm{m} / \mathrm{z}$ calculated for $\left[\mathrm{C}_{10} \mathrm{H}_{7} \mathrm{~N}_{2} \mathrm{SCl}_{3} \mathrm{Na}\right]^{+}\left([\mathrm{M}+\mathrm{Na}]^{+}\right)$: 316.9258 , measured: 316.9261 .

Methyl 2-((4-chloro-1-methyl-1H-imidazol-5-yl)thio)benzoate (3af)<smiles>COC(=O)c1ccccc1Sc1c(Cl)ncn1C</smiles>

Prepared from 4,5-dichloro-1-methyl-1H-imidazole $(45.3 \mathrm{mg}, 0.30 \mathrm{mmol})$ and methyl thiosalicylate $(62 \mu \mathrm{L}, 0.45 \mathrm{mmol})$ following general procedure A. The pure product was obtained after column chromatography (pentane:EtOAc $=3: 2-1: 2$ ) as a yellowish solid (65.0 mg, 77\%).

${ }^{1}$ H NMR (300 MHz, Chloroform-d) $\delta 8.04$ (dd, $\left.J=7.8,1.6 \mathrm{~Hz}, 1 \mathrm{H}\right), 7.66$ (s, 1H), 7.31 (ddd, $J$ $=8.1,7.3,1.6 \mathrm{~Hz}, 1 \mathrm{H}), 7.18(\mathrm{ddd}, J=7.7,7.3,1.2 \mathrm{~Hz}, 1 \mathrm{H}), 6.52(\mathrm{dd}, J=8.1,1.2 \mathrm{~Hz}, 1 \mathrm{H}), 3.96$ (s, 3H), $3.54(\mathrm{~d}, J=0.6 \mathrm{~Hz}, 3 \mathrm{H}) ;{ }^{13} \mathbf{C}$ NMR (75 MHz, Chloroform-d) $\delta 166.7,140.5,139.4$, 139.0, 133.2, 131.9, 126.4, 125.3, 125.2, 115.7, 52.5, 33.0; $\mathbf{R}_{\mathbf{f}}$ (pentane:EtOAc = 1:1): 0.15; HR-MS (ESI) $\mathrm{m} / \mathrm{z}$ calculated for $\left[\mathrm{C}_{12} \mathrm{H}_{11} \mathrm{~N}_{2} \mathrm{O}_{2} \mathrm{SClNa}\right]^{+}\left([\mathrm{M}+\mathrm{Na}]^{+}\right)$: 305.0122, measured: 305.0137 .

4-Chloro-1-methyl-5-(phenylthio)-1H-imidazole (3ag)<smiles>Cn1cnc(Cl)c1Sc1ccccc1</smiles>

Prepared from 4,5-dichloro-1-methyl- $1 \mathrm{H}$-imidazole $(45.3 \mathrm{mg}, 0.30 \mathrm{mmol})$, thiophenol $(93 \mu \mathrm{L}$, $0.90 \mathrm{mmol}, 3.0$ equiv) and $\left[\operatorname{Ir}\left(\mathrm{dFCF}_{3} \text { ppy }\right)_{2}(\mathrm{dtbbpy}) \mathrm{PF}_{6}(13.4 \mathrm{mg}, 4 \mathrm{~mol} \%)\right.$ following general procedure A. The pure product was obtained after column chromatography (pentane:EtOAc = $3: 2-1: 1)$ as a colorless oil (57.4 $\mathrm{mg}, 85 \%)$.

${ }^{1}$ H NMR (300 MHz, Chloroform-d) $\delta 7.58(\mathrm{~s}, 1 \mathrm{H}), 7.30-7.22(\mathrm{~m}, 2 \mathrm{H}), 7.21-7.14(\mathrm{~m}, 1 \mathrm{H})$, $7.11-7.05$ (m, 2H), 3.58 (s, 3H); ${ }^{13} \mathbf{C}$ NMR (75 MHz, Chloroform-d) $\delta$ 138.6, 138.5, 135.2, 129.5, 127.0, 126.7 116.2, 33.0; $\mathbf{R}_{\mathbf{f}}$ (pentane:EtOAc = 1:1): 0.22; HR-MS (ESI) m/z calculated for $\left[\mathrm{C}_{10} \mathrm{H}_{9} \mathrm{~N}_{2} \mathrm{SClNa}\right]^{+}\left([\mathrm{M}+\mathrm{Na}]^{+}\right): 247.0067$, measured: 247.0082 .

4-Chloro-1-methyl-5-( $p$-tolylthio)-1H-imidazole (3ah)<smiles>Cc1ccc(Sc2c(Cl)ncn2C)cc1</smiles> 
Prepared from 4,5-dichloro-1-methyl- $1 H$-imidazole $(45.3 \mathrm{mg}, \quad 0.30 \mathrm{mmol})$ and $p$-tolyl mercaptan (56 mg, $0.45 \mathrm{mmol}$ ) following general procedure A. The pure product was obtained after column chromatography (toluene:EtOAc $=4: 1$ ) as a white solid $(29.7 \mathrm{mg}, 41 \%)$.

${ }^{1}$ H NMR (400 MHz, Chloroform-d) $\delta 7.55(\mathrm{~s}, 1 \mathrm{H}), 7.07(\mathrm{~d}, J=8.2 \mathrm{~Hz}, 2 \mathrm{H}), 7.02(\mathrm{~d}, J=8.4$ $\mathrm{Hz}, 2 \mathrm{H}), 3.57$ (s, 3H), 2.29 (s, 3H); ${ }^{13} \mathrm{C}$ NMR (101 MHz, Chloroform-d) $\delta$ 138.31, 138.25, 136.9, 131.5, 130.3, 127.6, 117.0, 33.0, 21.1; $\mathbf{R}_{\mathbf{f}}$ (toluene:EtOAc = 7:3): 0.27; HR-MS (ESI) $\mathrm{m} / \mathrm{z}$ calculated for $\left[\mathrm{C}_{11} \mathrm{H}_{11} \mathrm{~N}_{2} \mathrm{SClNa}\right]^{+}\left([\mathrm{M}+\mathrm{Na}]^{+}\right): 261.0224$, measured: 261.0233 .

$N$-(4-((4-chloro-1-methyl-1H-imidazol-5-yl)thio)phenyl)-2,2,2-trifluoroacetamide (3ai)<smiles>Cn1cnc(Cl)c1Sc1ccc(NC(=O)C(F)(F)F)cc1</smiles>

Prepared from 4,5-dichloro-1-methyl- $1 \mathrm{H}$-imidazole $(45.3 \mathrm{mg}, 0.30 \mathrm{mmol})$ and 2,2,2-trifluoro$\mathrm{N}$-(4-mercaptophenyl)acetamide $(99.5 \mathrm{mg}, 0.45 \mathrm{mmol})$ following general procedure A. The pure product was obtained after column chromatography (pentane:EtOAc $=1: 1$ ) as a yellowish solid (61.2 mg, 61\%).

${ }^{1}$ H NMR (400 MHz, Methanol-d 4 ) $\delta 7.84(\mathrm{~s}, 1 \mathrm{H}), 7.60(\mathrm{~d}, J=8.8 \mathrm{~Hz}, 2 \mathrm{H}), 7.13(\mathrm{~d}, J=8.8 \mathrm{~Hz}$, 2H), $3.63(\mathrm{~s}, 3 \mathrm{H}) ;{ }^{13} \mathbf{C}$ NMR (101 MHz, Methanol-d 4 ) $\delta 156.7$ (q, $\left.J=37.6 \mathrm{~Hz}\right), 140.7,138.4$, 136.7, 133.3, 128.9, 123.2, 117.7, 117.4 (q, $J=288.3 \mathrm{~Hz}), 33.3 ;{ }^{19} \mathbf{F}$ NMR $(376 \mathrm{MHz}$, Methanol-d4) $\delta$-77.1; Rf (pentane:EtOAc = 1:1): 0.22; HR-MS (ESI) $\mathrm{m} / \mathrm{z}$ calculated for $\left[\mathrm{C}_{12} \mathrm{H}_{9} \mathrm{~N}_{3} \mathrm{OSClF}_{3} \mathrm{Na}\right]^{+}\left([\mathrm{M}+\mathrm{Na}]^{+}\right): 357.9999$, measured: 357.9996.

$N$-(2-((4-chloro-1-methyl-1H-imidazol-5-yl)thio)ethyl)-5-((3aS,4S,6aR)-2-oxohexahydro-1Hthieno[3,4- $d$ ] imidazol-4-yl)pentanamide (3aj)

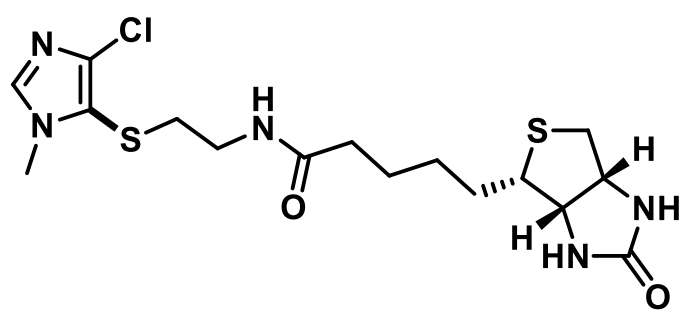

Prepared from 4,5-dichloro-1-methyl- $1 H$-imidazole $\quad(45.3 \mathrm{mg}, \quad 0.30 \mathrm{mmol})$ and $\mathrm{N}$-(2mercaptoethyl)-5-((3aS,4S,6aR)-2-oxohexahydro-1H-thieno[3,4- $d$ ]imidazol-4-yl)pentanamide (136.5 mg, $0.45 \mathrm{mmol}, 1.5$ equiv) following general procedure A. No aqueous work-up was performed due to water solubility of the product. Instead, $\mathrm{NaHCO}_{3}(176 \mathrm{mg}, 0.90 \mathrm{mmol}$, 3.0 equiv) was added to the crude reaction mixture and DMA was removed by co-evaporation with toluene (5 times). The pure product was obtained after column chromatography $\left(\mathrm{CH}_{2} \mathrm{Cl}_{2}: \mathrm{MeOH}=8: 1\right)$ as a white solid $(81.6 \mathrm{mg}, 65 \%)$.

${ }^{1}$ H NMR (400 MHz, Methanol-d 4$) \delta 7.75(\mathrm{~s}, 1 \mathrm{H}), 4.49(\mathrm{dd}, J=7.9,5.0 \mathrm{~Hz}, 1 \mathrm{H}), 4.30$ (dd, $J=$ 7.9, $4.5 \mathrm{~Hz}, 1 \mathrm{H}), 3.72$ (s, 3H), $3.36-3.30(\mathrm{~m}, 2 \mathrm{H}), 3.20$ (ddd, $J=8.9,5.8,4.4 \mathrm{~Hz}, 1 \mathrm{H}), 2.92$ $(\mathrm{dd}, J=12.7,5.0 \mathrm{~Hz}, 1 \mathrm{H}), 2.81(\mathrm{t}, J=7.0 \mathrm{~Hz}, 2 \mathrm{H}), 2.70(\mathrm{~d}, J=12.7 \mathrm{~Hz}, 1 \mathrm{H}), 2.19(\mathrm{t}, J=7.4$ 
$\mathrm{Hz}, 2 \mathrm{H}), 1.80-1.52(\mathrm{~m}, 4 \mathrm{H}), 1.49-1.37$ (m, 2H); ${ }^{13} \mathbf{C}$ NMR (101 MHz, Methanol-d4) $\delta$ 176.1, 166.1, 139.9, 137.1, 119.2, 63.3, 61.6, 57.0, 41.1, 40.3, 36.7, 35.7, 33.4, 29.7, 29.5, 26.7; $\mathbf{R} \mathbf{f}$ $\left(\mathrm{CH}_{2} \mathrm{Cl}_{2}: \mathrm{MeOH}=7: 1\right): 0.21$; HR-MS (ESI) $\mathrm{m} / \mathrm{z}$ calculated for $\left[\mathrm{C}_{16} \mathrm{H}_{24} \mathrm{~N}_{5} \mathrm{O}_{2} \mathrm{~S}_{2} \mathrm{ClNa}\right]^{+}$ $\left([\mathrm{M}+\mathrm{Na}]^{+}\right):$440.0952, measured: 440.0948 .

(2R,3R,4S,5R,6S)-2-(Acetoxymethyl)-6-((4-chloro-1-methyl-1H-imidazol-5yl)thio)tetrahydro-2 $H$-pyran-3,4,5-triyl triacetate (3ak)

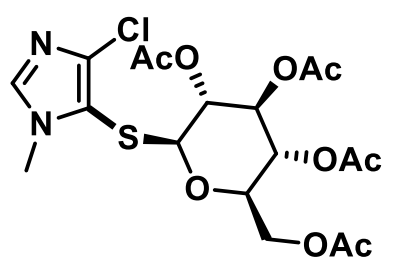

Prepared from 4,5-dichloro-1-methyl- $1 H$-imidazole $(45.3 \mathrm{mg}, 0.30 \mathrm{mmol})$ and 1-thio- $\beta$ - $D$ glucose tetraacetate $(164 \mathrm{mg}, 0.45 \mathrm{mmol}$ ) following general procedure A. The pure product was obtained after column chromatography (pentane:EtOAc $=1: 1-$ EtOAc) as a white solid (138.5 mg, 96\%).

${ }^{1} \mathbf{H}$ NMR (400 MHz, Chloroform-d) $\delta 7.47(\mathrm{~s}, 1 \mathrm{H}), 5.16(\mathrm{t}, J=9.4 \mathrm{~Hz}, 1 \mathrm{H}), 4.99(\mathrm{t}, J=9.8 \mathrm{~Hz}$, $1 \mathrm{H}), 4.91(\mathrm{t}, J=9.7 \mathrm{~Hz}, 1 \mathrm{H}), 4.46(\mathrm{~d}, J=10.2 \mathrm{~Hz}, 1 \mathrm{H}), 4.14-4.06(\mathrm{~m}, 2 \mathrm{H}), 3.64(\mathrm{~s}, 3 \mathrm{H}), 3.59$ - $3.52(\mathrm{~m}, 1 \mathrm{H}), 2.09$ (s, 3H), $2.01(\mathrm{~s}, 3 \mathrm{H}), 1.98$ (s, 3H), $1.96(\mathrm{~s}, 3 \mathrm{H}) ;{ }^{13} \mathbf{C}$ NMR $(101 \mathrm{MHz}$, Chloroform-d) $\delta 170.4,170.1,169.6,169.4,138.8,138.3,115.0,87.1,75.9,73.6,70.1,68.0$, 62.0, 33.3, 20.8, 20.7, 20.61, 20.58; Rf (EtOAc): 0.32; HR-MS (ESI) m/z calculated for $\left[\mathrm{C}_{18} \mathrm{H}_{23} \mathrm{~N}_{2} \mathrm{O}_{9} \mathrm{SClNa}\right]^{+}\left([\mathrm{M}+\mathrm{Na}]^{+}\right): 501.0705$, measured: 501.0714 .

4-Chloro-5-(((3R,5S,8R,9S,10S,13R,14S,17R)-10,13-dimethyl-17-((R)-6-methylheptan-2yl)hexadecahydro- $1 H$-cyclopenta[ $a]$ phenanthren-3-yl)thio)-1-methyl-1 $H$-imidazole (3al)

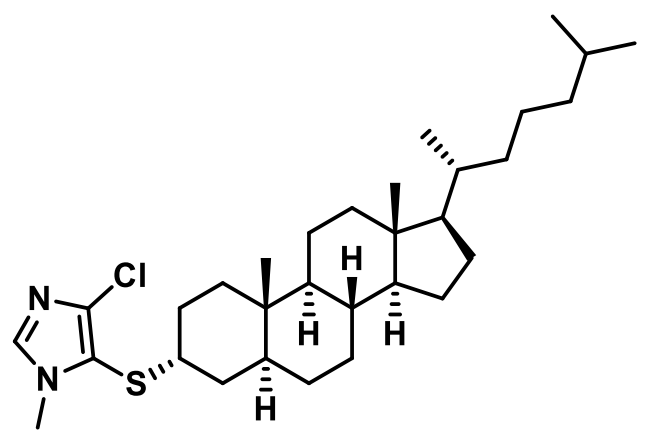

Prepared from 4,5-dichloro-1-methyl-1H-imidazole $\quad(45.3 \mathrm{mg}, \quad 0.30 \mathrm{mmol}) \quad$ and $(3 R, 5 S, 8 R, 9 S, 10 S, 13 R, 14 S, 17 R)-10,13$-dimethyl-17-( $R)-6$-methylheptan-2-

yl)hexadecahydro- $1 H$-cyclopenta[$[a]$ phenanthrene-3-thiol $(182.1 \mathrm{mg}, 0.45 \mathrm{mmol})$ following general procedure A using a toluene/DMA solvent mixture $(1: 1,3.0 \mathrm{~mL})$. The pure product was obtained after column chromatography (pentane:EtOAc $=1: 1)$ as a yellowish solid $(93.0 \mathrm{mg}$, $60 \%)$.

${ }^{1}$ H NMR (400 MHz, Chloroform-d) $\delta 7.45$ (s, 1H), 3.64 (s, 3H), 3.40 - 3.33 (m, 1H), 1.97 (dt, $J=12.3,3.2 \mathrm{~Hz}, 1 \mathrm{H}), 1.88-1.41(\mathrm{~m}, 11 \mathrm{H}), 1.38-0.73(\mathrm{~m}, 31 \mathrm{H}), 0.64(\mathrm{~s}, 3 \mathrm{H}) ;{ }^{13} \mathbf{C ~ N M R}(101$ 
MHz, Chloroform-d) $\delta 137.6,119.1,56.6,56.4,54.3,48.0,42.7,40.4,40.1,39.6,36.5,36.3$, 35.9, 35.6, 33.2, 33.0, 32.9, 32.0, 28.5, 28.4, 28.1, 26.5, 24.3, 24.0, 23.0, 22.7, 20.9, 18.8, 12.2, 11.9 (one signal missing); Rf (pentane:EtOAc = 1:1): 0.18; HR-MS (ESI) m/z calculated for $\left[\mathrm{C}_{31} \mathrm{H}_{51} \mathrm{~N}_{2} \mathrm{SClNa}\right]^{+}\left([\mathrm{M}+\mathrm{Na}]^{+}\right): 541.3354$, measured: 541.3346.

Methyl $\quad N^{2}$-(tert-butoxycarbonyl)- $N^{4}$-(2-((4-chloro-1-methyl-1H-imidazol-5-yl)thio)ethyl)- $L$ asparaginyl- $L$-phenylalaninate (3am)<smiles>COC(=O)C(Cc1ccccc1)NC(=O)C(CC(=O)NCCSc1c(Cl)ncn1C)NC(=O)O</smiles>

Prepared from 4,5-dichloro-1-methyl- $1 \mathrm{H}$-imidazole $(45.3 \mathrm{mg}, 0.30 \mathrm{mmol})$ and methyl $\mathrm{N}^{2}$-(tertbutoxycarbonyl)- $N^{4}$-(2-mercaptoethyl)- $L$-asparaginyl- $L$-phenylalaninate $(204 \mathrm{mg}, 0.45 \mathrm{mmol}$ ) following general procedure A. The pure product was obtained after column chromatography $\left(\mathrm{CH}_{2} \mathrm{Cl}_{2}: \mathrm{MeOH}=20: 1\right)$ as a yellowish solid $(153 \mathrm{mg}, 90 \%)$.

${ }^{1}$ H NMR (300 MHz, Chloroform-d) $\delta 7.46(\mathrm{~s}, 1 \mathrm{H}), 7.43-7.34(\mathrm{~m}, 1 \mathrm{H}), 7.32-7.19(\mathrm{~m}, 3 \mathrm{H})$, $7.15-7.08(\mathrm{~m}, 2 \mathrm{H}), 6.77-6.62(\mathrm{~m}, 1 \mathrm{H}), 6.06(\mathrm{~d}, J=7.9 \mathrm{~Hz}, 1 \mathrm{H}), 4.80-4.64(\mathrm{~m}, 1 \mathrm{H}), 4.48-$ $4.34(\mathrm{~m}, 1 \mathrm{H}), 3.65(\mathrm{~s}, 3 \mathrm{H}), 3.62(\mathrm{~s}, 3 \mathrm{H}), 3.41-3.21(\mathrm{~m}, 2 \mathrm{H}), 3.06(\mathrm{dd}, J=6.1,2.8 \mathrm{~Hz}, 2 \mathrm{H})$, $2.82-2.66(\mathrm{~m}, 3 \mathrm{H}), 2.51(\mathrm{dd}, J=15.5,6.2 \mathrm{~Hz}, 1 \mathrm{H}), 1.39(\mathrm{~s}, 9 \mathrm{H}) ;{ }^{13} \mathbf{C}$ NMR $(75 \mathrm{MHz}$, Chloroform-d) $\delta 171.6,171.2,171.1,155.7,138.1,137.4,135.9,129.3,128.7,127.2,117.2$, 80.3, 53.7, 52.4, 51.0, 39.1, 37.9, 37.6, 35.2, 32.9, 28.3; $\mathbf{R}_{\mathbf{f}}\left(\mathrm{CH}_{2} \mathrm{Cl}_{2}: \mathrm{MeOH}=20: 1\right): 0.21$; HRMS (ESI) $\mathrm{m} / \mathrm{z}$ calculated for $\left[\mathrm{C}_{25} \mathrm{H}_{34} \mathrm{~N}_{5} \mathrm{O}_{6} \mathrm{SClNa}\right]^{+}\left([\mathrm{M}+\mathrm{Na}]^{+}\right): 590.1811$, measured: 590.1822 .

Methyl $N^{2}$-(tert-butoxycarbonyl)- $N^{5}$-((R)-3-((4-chloro-1-methyl-1H-imidazol-5-yl)thio)-1-((2methoxy-2-oxoethyl)amino)-1-oxopropan-2-yl)- $L$-glutaminate (3an)<smiles>COC(=O)CNC(=O)[C@H](CSc1c(Cl)ncn1C)NC(=O)CC[C@H](NC(C)(C)C)C(=O)O</smiles>

Prepared from 4,5-dichloro-1-methyl- $1 \mathrm{H}$-imidazole $(45.3 \mathrm{mg}, 0.30 \mathrm{mmol})$ and methyl $\mathrm{N}^{2}$-(tertbutoxycarbonyl)- $N^{5}$-((R)-3-mercapto-1-((2-methoxy-2-oxoethyl)amino)-1-oxopropan-2-yl)- $L$ glutaminate $(196 \mathrm{mg}, 0.45 \mathrm{mmol})$ following general procedure A. The pure product was obtained after column chromatography (DCM - DCM:MeOH = 9:1) as a yellowish solid (94.4 mg, 57\%).

${ }^{1} \mathbf{H}$ NMR (300 MHz, Chloroform-d) $\delta 7.71-7.60(\mathrm{~m}, 1 \mathrm{H}), 7.56(\mathrm{~s}, 1 \mathrm{H}), 7.18(\mathrm{~d}, J=7.8 \mathrm{~Hz}$, $1 \mathrm{H}), 5.47(\mathrm{~d}, J=8.2 \mathrm{~Hz}, 1 \mathrm{H}), 4.60-4.48(\mathrm{~m}, 1 \mathrm{H}), 4.40-4.29(\mathrm{~m}, 1 \mathrm{H}), 3.90(\mathrm{~d}, J=5.2 \mathrm{~Hz}$, 2H), $3.72-3.64(\mathrm{~m}, 9 \mathrm{H}), 3.22-2.96(\mathrm{~m}, 2 \mathrm{H}), 2.35(\mathrm{t}, J=7.1 \mathrm{~Hz}, 2 \mathrm{H}), 2.24-2.08(\mathrm{~m}, 1 \mathrm{H})$, 
$1.99-1.83(\mathrm{~m}, 1 \mathrm{H}), 1.39$ (s, 9H); ${ }^{13} \mathrm{C}$ NMR (75 MHz, Chloroform-d) $\delta$ 173.0, 172.5, 170.1, 170.0, 155.9, 138.2, 136.3, 117.1, 80.2, 52.8, 52.6, 52.4, 41.3, 36.5, 33.1, 32.1, 28.5, 28.3 (one signal missing); Rf (DCM:MeOH = 19:1): 0.06; HR-MS (ESI) $\mathrm{m} / \mathrm{z}$ calculated for $\left[\mathrm{C}_{21} \mathrm{H}_{32} \mathrm{~N}_{5} \mathrm{O}_{8} \mathrm{SClH}\right]^{+}\left([\mathrm{M}+\mathrm{H}]^{+}\right): 550.1733$, measured: 550.1744. 


\subsection{Methylthiolation of Halogenated Heteroarenes}

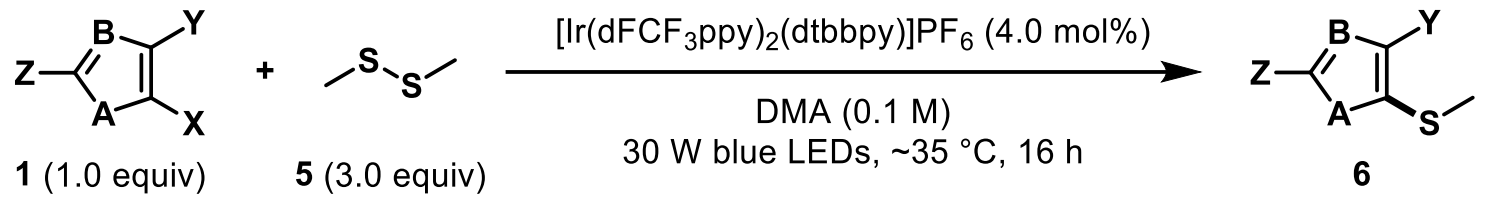

\section{General Procedure D}

Unless otherwise noted, the corresponding heteroarene $(0.30 \mathrm{mmol}, 1.0$ equiv $)$ and [Ir(dFCF 3 ppy $\left.)_{2}(\mathrm{dtbbpy})\right] \mathrm{PF}_{6}(13.4 \mathrm{mg}, 0.012 \mathrm{mmol}, 4.0 \mathrm{~mol} \%)$ were weighed into a Schlenk tube containing a magnetic stirring bar. The tube was evacuated and backfilled with argon three times. DMA (3 mL, 0.1 M) was added, followed by dimethyl disulfide $(81 \mu \mathrm{L}, 0.90 \mathrm{mmol}$, 3.0 equiv). The reaction was stirred under irradiation with $455 \mathrm{~nm}$ LEDs for $16 \mathrm{~h}$ at room temperature. The crude mixture was diluted with EtOAc. $\mathrm{NaHCO}_{3}$-solution (sat., $10 \mathrm{~mL}$ ) and water $(10 \mathrm{~mL})$ were added, the layers were separated, and the organic layer was washed with water $(2 \times 20 \mathrm{~mL})$ and dried over $\mathrm{Na}_{2} \mathrm{SO}_{4}$. The solvent was removed under reduced pressure and the crude product was purified by column chromatography over silica gel to afford analytically pure products.

4-Chloro-1-methyl-5-(methylthio)-1H-imidazole (6a)<smiles>CSc1c(Cl)ncn1C</smiles>

Prepared from 4,5-dichloro-1-methyl-1H-imidazole $(45.3 \mathrm{mg}, 0.30 \mathrm{mmol})$ following general procedure D. The pure product was obtained after column chromatography (pentane:EtOAc = $1: 1-1: 2)$ as a yellowish oil (40.3 $\mathrm{mg}, 83 \%)$.

${ }^{1} \mathbf{H}$ NMR (400 MHz, Chloroform-d) $\delta 7.49$ (s, 1H), 3.68 (s, 3H), 2.26 (s, 3H); ${ }^{13}$ C NMR (101 MHz, Chloroform-d) $\delta 137.4,136.2,119.9,32.9,19.2$; $\mathbf{R}_{\mathbf{f}}$ (pentane:EtOAc = 1:1): 0.20; HRMS (ESI) $\mathrm{m} / \mathrm{z}$ calculated for $\left[\mathrm{C}_{5} \mathrm{H}_{7} \mathrm{~N}_{2} \mathrm{SClNa}\right]^{+}\left([\mathrm{M}+\mathrm{Na}]^{+}\right)$: 184.9922 , measured: 184.9925 .

\section{2,4-Dibromo-1-methyl-5-(methylthio)-1H-imidazole (6b)}<smiles>CSc1c(Br)nc(Br)n1C</smiles>

Prepared from 2,4,5-tribromo-1-methyl- $1 \mathrm{H}$-imidazole $(95.6 \mathrm{mg}, 0.30 \mathrm{mmol})$ following general procedure D. The pure product was obtained after column chromatography (pentane:Et $2 \mathrm{O}=$ 4:1) as a white solid (44.5 $\mathrm{mg}, 52 \%)$.

${ }^{1} \mathbf{H}$ NMR (300 MHz, Chloroform-d) $\delta 3.68(\mathrm{~s}, 3 \mathrm{H}), 2.26(\mathrm{~s}, 3 \mathrm{H}) ;{ }^{13} \mathbf{C}$ NMR $(75 \mathrm{MHz}$, Chloroform-d) $\delta 126.0,123.8,120.8,33.8,19.5$; $\mathbf{R}_{\mathbf{f}}$ (pentane: $\mathrm{Et}_{2} \mathrm{O}=4: 1$ ): 0.16; HR-MS (ESI) $\mathrm{m} / \mathrm{z}$ calculated for $\left[\mathrm{C}_{5} \mathrm{H}_{6} \mathrm{Br}_{2} \mathrm{~N}_{2} \mathrm{SNa}\right]^{+}\left([\mathrm{M}+\mathrm{Na}]^{+}\right)$: 308.8490 , measured: 308.8497 . 
5-(Methylthio)-4-phenylthiazol-2-amine (6c)<smiles>CSc1sc(N)nc1-c1ccccc1</smiles>

Prepared from 5-chloro-4-phenylthiazol-2-amine $(63 \mathrm{mg}, 0.30 \mathrm{mmol})$ following general procedure D. The pure product was obtained after column chromatography (pentane:EtOAc $=$ $3: 1-2: 1)$ as a yellowish oil (31.0 $\mathrm{mg}, 46 \%)$.

${ }^{1}$ H NMR (300 MHz, Chloroform-d) $\delta 7.88-7.80$ (m, 2H), 7.46 - 7.30 (m, 3H), 5.50 (s, 2H), 2.32 (s, 3H); ${ }^{13} \mathbf{C}$ NMR (75 MHz, Chloroform-d) $\delta 167.5,153.2,134.3,128.9,128.2,128.2$, 114.4, 22.3; $\mathbf{R}_{\mathbf{f}}$ (pentane:EtOAc = 2:1): 0.27; HR-MS $\left(\right.$ ESI) $\mathrm{m} / \mathrm{z}$ calculated for $\left[\mathrm{C}_{10} \mathrm{H}_{10} \mathrm{~N}_{2} \mathrm{~S}_{2} \mathrm{H}\right]^{+}$ $\left([\mathrm{M}+\mathrm{H}]^{+}\right):$223.0358, measured: 223.0375. 


\subsection{Limitation Studies}

A summary of substrates which were found to be unreactive under our reaction conditions is given in Figure S5. Electron-rich 5-membered heteroarenes bearing halogen substituents only in $\mathrm{C} 3 / \mathrm{C} 4$ position did not show any conversion to the respective product. This explains the high selectivity observed for $\mathrm{C} 2 / \mathrm{C} 5$ over $\mathrm{C} 3 / \mathrm{C} 4$ substitution. Arenes in general, as included in the substrate scope, as well as more electron-rich halogenated benzenes did not react but were tolerated under the thiolation protocol, underlining its complementarity to established coupling methods (e.g. metal-catalyzed cross-coupling). Furthermore, less electron-rich 5-membered heteroarenes, such as pyrazoles and triazoles, did not show conversion and sterically congested tertiary thiols were found to be unreactive.

a) $\mathrm{C} 3 / \mathrm{C} 4$ halogenated electron-rich heteroarenes<smiles>Cn1cnc(Br)c1</smiles><smiles>[AlH2]n1ccc(Br)c1</smiles><smiles>Brc1ccsc1</smiles><smiles>Brc1cscc1Br</smiles><smiles>Brc1ccoc1</smiles>

b) Arenes<smiles>COc1ccc(Br)cc1</smiles><smiles>COc1ccc(Br)cc1OC</smiles><smiles>COc1ccccc1Cl</smiles>

c) Unreactive 5-membered heteroarenes<smiles>Cn1nc(Br)c(Br)c1Br</smiles><smiles>Brc1nnn(Cc2ccccc2)c1Br</smiles><smiles>Brc1nc(Br)n(Cc2ccccc2)n1</smiles>

d) Tertiary thiols<smiles>[13CH3][13CH3]</smiles>

Figure S5. Summary of unreactive substrates (no conversion was observed). 


\subsection{Additive-Based Robustness Screen}

The robustness and the functional group preservation of the thiolation of heteroarenes were investigated by applying an intermolecular additive-based screen. This screening methodology, reported by our group in 2014, evaluates the tolerance of the present reaction to a series of additives (robustness), as well as the stability of these additives to the reaction conditions (functional group preservation). ${ }^{24}$

The general procedure of such a screening involves the equimolar addition of a single functionalized additive to the standard reaction conditions. The yield of the product as well as the relative amount of remaining additive is determined by GC-FID analysis. Calibration of the additives is done using a single point batch calibration.

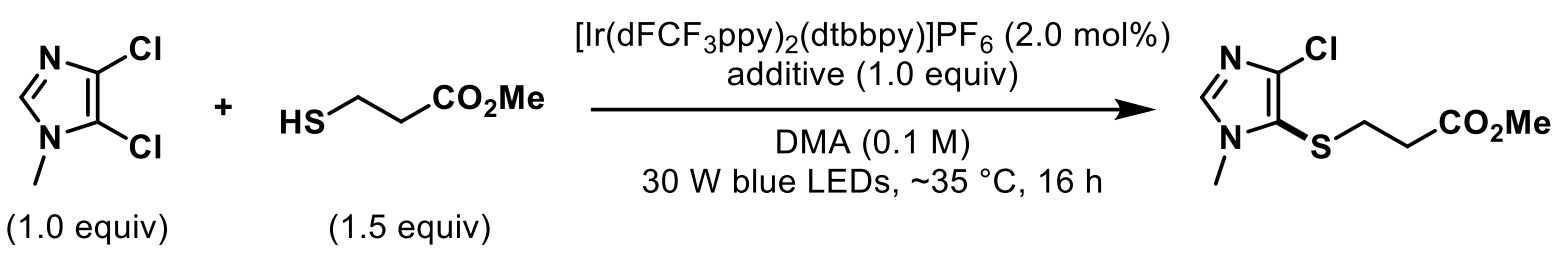

Conditions: $n=0.1 \mathrm{mmol}, c=0.1 \mathrm{M}, V=1.0 \mathrm{~mL}$, inert atmosphere, $T=35^{\circ} \mathrm{C}$.

Stock solution: $n=1.6 \mathrm{mmol}, c=0.1 \mathrm{M}, V=16 \mathrm{~mL}, 4,5$-dichloro-1-methyl- $1 H$-imidazole: $242 \mathrm{mg}$, methyl 3-mercaptopropanoate: $266 \mu \mathrm{L}$, [Ir(dFCF 3 ppy $)_{2}(\mathrm{dtbbpy}) \mathrm{PF}_{6}: 35.2 \mathrm{mg}$, DMA: $16 \mathrm{~mL}$, mesitylene: $221 \mu \mathrm{L}$ (1.0 equiv).

The stock solution was prepared under inert atmosphere. The respective additive $(0.1 \mathrm{mmol}$, 1.0 equiv) was placed in a Schlenk tube containing a magnetic stirring bar under argon atmosphere. Stock solution $(1.0 \mathrm{~mL})$ was added and the reaction was stirred under irradiation with $455 \mathrm{~nm}$ LEDs for $16 \mathrm{~h}$ at room temperature. The crude mixture was diluted with EtOAc. $\mathrm{NaHCO}_{3}$-solution (sat., $3 \mathrm{~mL}$ ) and water $(3 \mathrm{~mL})$ were added and the layers were separated. An aliquot $(100 \mu \mathrm{L})$ of the organic layer was filtered over a plug of silica (elution with EtOAc) and the yield as well as the relative amount of remaining additive were determined via calibrated GC-FID. In case of acetanilide as additive, the GC sample was prepared by filtration over Celite. The results are displayed in Table S4. 
Table S4. Results of the additive-based robustness screen.

\begin{tabular}{|c|c|c|c|}
\hline Entry & Additive & Yield Additive / \% & Yield Product / \% \\
\hline 1 & & 70 & 88 \\
\hline 2 & & 0 & 34 \\
\hline 3 & & 78 & 84 \\
\hline 4 & & 1 & 47 \\
\hline 5 & & 84 & 50 \\
\hline 6 & & 86 & 99 \\
\hline 7 & & 96 & 86 \\
\hline 8 & & 80 & 62 \\
\hline 9 & & 95 & 88 \\
\hline 10 & & 95 & 83 \\
\hline 11 & & 70 & 100 \\
\hline 12 & & 99 & 98 \\
\hline 13 & & 42 & 83 \\
\hline 14 & & 36 & 83 \\
\hline 15 & & 0 & 83 \\
\hline 16 & none & none & 92 \\
\hline
\end{tabular}

62 
Color-coding should help the ready assessment of the yields: green $(>66 \%)$, yellow $(34 \ldots 66 \%)$, red $(<34 \%)$ for the additive recovery. The color-coding for facilitated assessment of the product yield is scaled using these percentages relative to the yield of the standard reaction in the absence of any additive, representing $>60 \%$ in green, $32 \ldots 60 \%$ in yellow and $<32 \%$ in red.

\subsection{Competition Experiment of Chlorinated and Brominated Substrates}

The relative reactivity of 4,5-dichloro-1-methyl- $1 \mathrm{H}$-imidazole and 4,5-dibromo-1-methyl- $1 \mathrm{H}$ imidazole was investigated in a competition experiment.

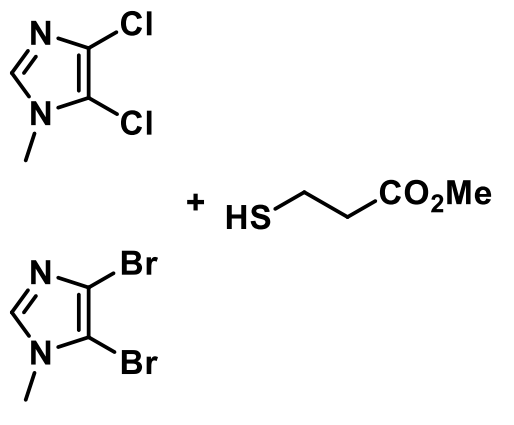

(1.0 equiv) (1.0 equiv)

$\left[\operatorname{lr}\left(\mathrm{dFCF}_{3} \mathrm{ppy}_{2}(\mathrm{dtbbpy})\right] \mathrm{PF}_{6}(2.0 \mathrm{~mol} \%)\right.$

DMA $(0.1 \mathrm{M})$

$30 \mathrm{~W}$ blue LEDs, $\sim 35^{\circ} \mathrm{C}, 16 \mathrm{~h}$

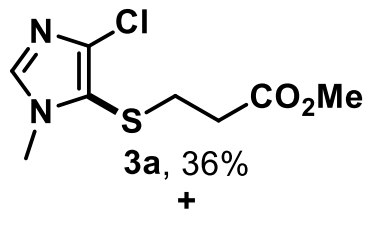<smiles>CC(=O)CCSc1c(Br)ncn1C</smiles>

3 b, $25 \%$

4,5-Dichloro-1-methyl-1H-imidazole ( $15.1 \mathrm{mg}, 0.10 \mathrm{mmol}, 1.0$ equiv), 4,5-dibromo-1-methyl$1 H$-imidazole $\left(24.0 \mathrm{mg}, 0.10 \mathrm{mmol}, 1.0\right.$ equiv) and $\left[\operatorname{Ir}\left(\mathrm{dFCF}_{3} \mathrm{ppy}\right)_{2}(\mathrm{dtbbpy}) \mathrm{PF}_{6}(2.2 \mathrm{mg}\right.$, $0.002 \mathrm{mmol}, 2.0 \mathrm{~mol} \%$ ) were weighed into a Schlenk tube containing a magnetic stirring bar. The tube was evacuated and backfilled with argon three times. DMA (1 mL, $0.1 \mathrm{M})$ was added, followed by methyl 3-mercaptopropanoate $(11.1 \mu \mathrm{L}, 0.30 \mathrm{mmol}, 1.0$ equiv) under a positive stream of argon. The reaction was stirred under irradiation with $455 \mathrm{~nm}$ LEDs for $16 \mathrm{~h}$ at room temperature. The crude mixture was diluted with EtOAc. Mesitylene $(13.8 \mu \mathrm{L}, 0.1 \mathrm{mmol}$, 1.0 equiv) was added as an internal standard and an aliquot $(100 \mu \mathrm{L})$ of the crude reaction mixture was filtered over a plug of silica (elution with EtOAc). The yield was determined via calibrated GC-FID.

Product 3a could be obtained in 36\% yield and product $\mathbf{3 b}$ in $25 \%$ yield, suggesting that the chlorinated imidazole reacts slightly faster than the brominated analogue. 


\section{Comparison to Established C-S Coupling Protocols}

In order to further show the complementary nature of the developed $\mathrm{C}-\mathrm{S}$ coupling protocol to established methods, we subjected our standard substrate 2a as well as the more demanding substrate $\mathbf{2 l}$ bearing an additional aryl bromide group to a palladium-catalyzed cross-coupling reaction. Additionally, a comparison to a nickel-photoredox dual-catalytic coupling was performed.<smiles>Cn1cnc(Cl)c1Cl</smiles>

$2 a$<smiles>Clc1ncn(Cc2ccc(Br)cc2)c1Cl</smiles>

2I

\subsection{Palladium-Catalyzed Cross-Coupling}

The thiolation protocol was compared to an established $\mathrm{C}-\mathrm{S}$ coupling protocol by Itoh et al. employing Xantphos as a chelating diphosphine ligand. ${ }^{6 \mathrm{~d}}$

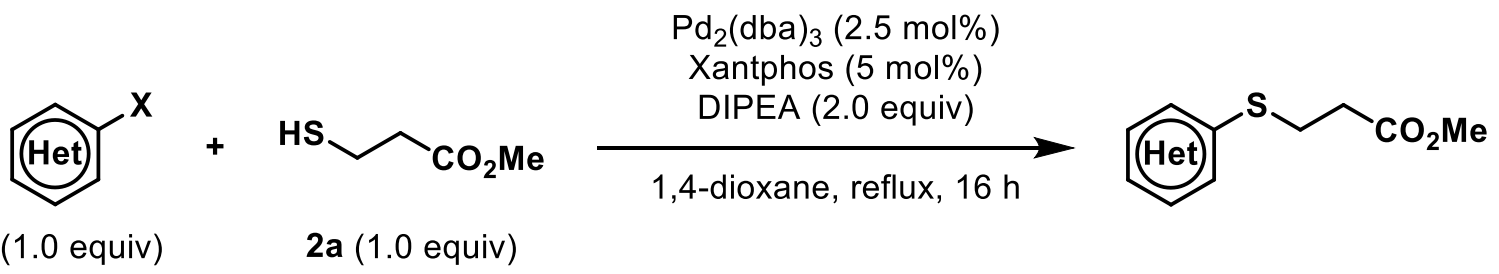

The corresponding heteroarene $\left(0.30 \mathrm{mmol}, 1.0\right.$ equiv), $\mathrm{Pd}_{2}(\mathrm{dba})_{3}(6.9 \mathrm{mg}, 0.0075 \mathrm{mmol}$, $2.5 \mathrm{~mol} \%)$ and Xantphos $(8.7 \mathrm{mg}, 0.015 \mathrm{mmol}, 5 \mathrm{~mol} \%)$ were weighed into a Schlenk tube containing a magnetic stirring bar. The tube was evacuated and backfilled with argon three times. 1,4-Dioxane $(0.6 \mathrm{~mL}, 0.5 \mathrm{M})$ was added, followed by methyl 3-mercaptopropanoate (33.2 $\mu \mathrm{L}, 0.30 \mathrm{mmol}, 1.0$ equiv) and $N, N$-diisopropylethylamine (DIPEA, $105 \mu \mathrm{L}, 0.6 \mathrm{mmol}$, 2.0 equiv). The reaction was heated to reflux for $16 \mathrm{~h}$. The crude mixture was allowed to cool to room temperature and the solvent was removed under reduced pressure. The crude product was purified by column chromatography over silica gel to afford analytically pure products.

No conversion was observed when reacting heteroarene 2a. Reaction of substrate $\mathbf{2 l}$ solely resulted in (undesired) substitution of the $\mathrm{C}-\mathrm{Br}$ bond.

Methyl 3-((4-((4,5-dichloro-1H-imidazol-1-yl)methyl)phenyl)thio)propanoate (4)<smiles>CC(=O)CCSc1ccc(Cn2cnc(Cl)c2Cl)cc1</smiles> 
Prepared from 1-(4-bromobenzyl)-4,5-dichloro-1H-imidazole $(91.8 \mathrm{mg}, 0.30 \mathrm{mmol})$. The pure product was obtained after column chromatography (pentane:EtOAc $=3: 2$ ) as a yellowish oil (100.5 mg, 97\%).

${ }^{1}$ H NMR (400 MHz, Chloroform-d) $\delta 7.37$ (s, 1H), $7.32-7.28(\mathrm{~m}, 2 \mathrm{H}), 7.10-7.05(\mathrm{~m}, 2 \mathrm{H})$, $5.01(\mathrm{~s}, 2 \mathrm{H}), 3.64(\mathrm{~s}, 3 \mathrm{H}), 3.14(\mathrm{t}, J=7.4 \mathrm{~Hz}, 2 \mathrm{H}), 2.60(\mathrm{t}, J=7.3 \mathrm{~Hz}, 2 \mathrm{H}) ;{ }^{13} \mathbf{C}$ NMR $(101$ MHz, Chloroform-d) $\delta 172.0,136.4,134.4,132.5,129.9,128.1,126.4,113.5,51.9,49.3,34.0$, 28.6; Rf (pentane:EtOAc = 3:2): 0.25; HR-MS (ESI) $\mathrm{m} / \mathrm{z}$ calculated for $\left[\mathrm{C}_{14} \mathrm{H}_{14} \mathrm{~N}_{2} \mathrm{O}_{2} \mathrm{SCl}_{2} \mathrm{Na}\right]^{+}$ $\left([\mathrm{M}+\mathrm{Na}]^{+}\right): 367.00453$, measured: 367.00434 .

\subsection{Nickel-Photoredox Dual-Catalytic Coupling}

The thiolation protocol was compared to a Nickel/photoredox-dual catalytic coupling reaction by Oderinde et al. ${ }^{17 a}$ It should be noted that the study uses the same photocatalyst as employed in our protocol. Therefore, product formation using the electron-rich heteroarene substrates might result via a background reaction without the need for the Nickel catalyst. Thus, control experiments without the Nickel catalyst and the ligand were also performed.

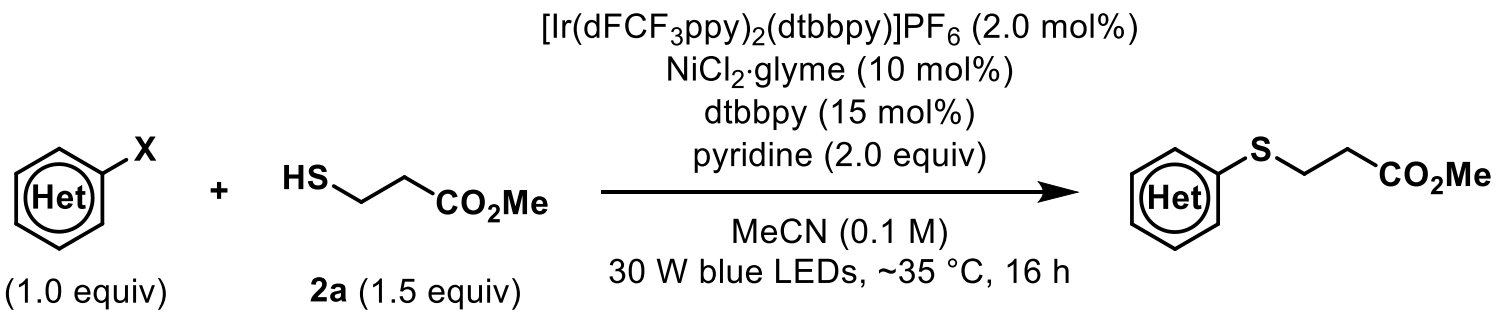

The corresponding heteroarene $\left(0.30 \mathrm{mmol}, 1.0\right.$ equiv), [ $\left.\operatorname{Ir}\left(\mathrm{dFCF}_{3} \mathrm{ppy}\right)_{2}(\mathrm{dtbbpy})\right] \mathrm{PF}_{6}(6.7 \mathrm{mg}$, $0.006 \mathrm{mmol}, 2.0 \mathrm{~mol} \%), \mathrm{NiCl}_{2} \cdot$ glyme $(6.6 \mathrm{mg}, 0.03 \mathrm{mmol}, 10 \mathrm{~mol} \%)$ and 4,4'-di-tert-butyl2,2'-dipyridyl (dtbbpy, $12.1 \mathrm{mg}, 0.045 \mathrm{mmol}, 15 \mathrm{~mol} \%$ ) were weighed into a Schlenk tube containing a magnetic stirring bar. The tube was evacuated and backfilled with argon three times. $\mathrm{MeCN}(3.0 \mathrm{~mL}, 0.1 \mathrm{M})$ was added, followed by methyl 3-mercaptopropanoate $(49.9 \mu \mathrm{L}$, $0.45 \mathrm{mmol}, 1.5$ equiv) and pyridine $(48.5 \mu \mathrm{L}, 0.6 \mathrm{mmol}, 2.0$ equiv). The reaction was stirred under irradiation with $455 \mathrm{~nm}$ LEDs for $16 \mathrm{~h}$ at room temperature. The solvent was removed under reduced pressure and the crude product was purified by column chromatography over silica gel to afford analytically pure products.

Heteroarene 2a reacted under formation of the desired product 3a. However, the reaction took place similarly when omitting the Nickel catalyst and the ligand. Reaction of substrate $2 \mathbf{l}$ resulted in formation of two (undesired) products both resulting from substitution of the $\mathrm{C}-\mathrm{Br}$ bond. 
Methyl 3-((4-chloro-1-methyl-1H-imidazol-5-yl)thio)propanoate (3a)<smiles>COC(=O)CCSc1c(Cl)ncn1C</smiles>

Prepared from 4,5-dichloro-1-methyl-1H-imidazole $(45.3 \mathrm{mg}, 0.30 \mathrm{mmol})$. The pure product was obtained after column chromatography (EtOAc) as a yellowish oil (69.9 mg, 99\%).

A control experiment without the Nickel catalyst and the dtbbpy ligand showed that solely the photocatalyst was needed for product formation (quantitative formation of $\mathbf{3 a}$, determined via calibrated GC-FID using mesitylene as an internal standard).

Methyl 3-((4-((4,5-dichloro-1H-imidazol-1-yl)methyl)phenyl)thio)propanoate (4) and methyl 3-((5-chloro-1-(4-((3-methoxy-3-oxopropyl)thio)benzyl)-1H-imidazol-4-yl)thio)propanoate (SI-1)

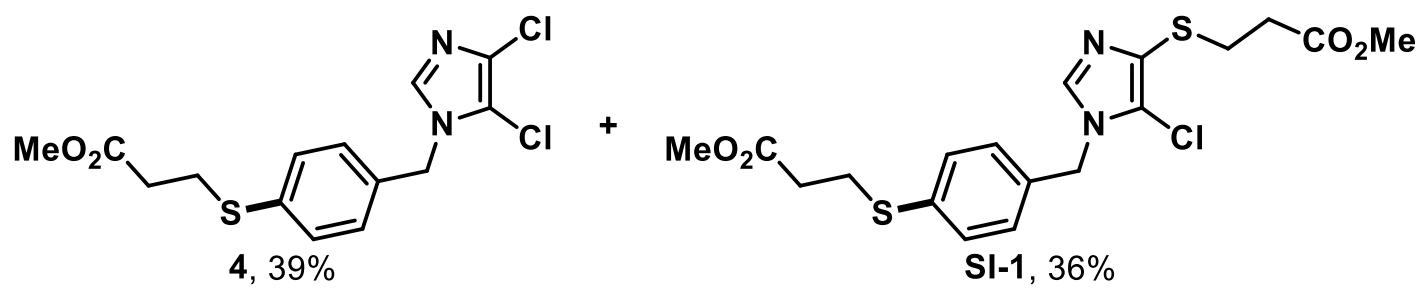

Prepared from 1-(4-bromobenzyl)-4,5-dichloro-1H-imidazole $\quad(91.8 \mathrm{mg}, \quad 0.30 \mathrm{mmol})$. Separation by column chromatography (pentane:EtOAc $=3: 2$ ) afforded $\mathbf{4}$ as a yellowish oil (40.1 $\mathrm{mg}, 39 \%$ ) and SI-1 as a yellowish oil (46.5 mg, 36\%).

Analytical data for SI-1:

${ }^{1}$ H NMR (300 MHz, Chloroform-d) $\delta 7.59(\mathrm{~s}, 1 \mathrm{H}), 7.31(\mathrm{~d}, J=8.3 \mathrm{~Hz}, 2 \mathrm{H}), 7.10(\mathrm{~d}, J=8.3$ $\mathrm{Hz}, 2 \mathrm{H}), 5.15$ (s, 2H), 3.66 (s, 3H), 3.63 (s, 3H), 3.15 (t, $J=7.4 \mathrm{~Hz}, 2 \mathrm{H}), 2.71$ (t, $J=7.0 \mathrm{~Hz}$, 2H), $2.60(\mathrm{t}, J=7.4 \mathrm{~Hz}, 2 \mathrm{H}), 2.50(\mathrm{t}, J=7.0 \mathrm{~Hz}, 2 \mathrm{H}) ; \mathbf{R}_{\mathbf{f}}($ pentane:EtOAc = 3:2): 0.19 .

\subsection{Summary of the Results}

A summary of the results is depicted in Figure S6. No reaction at the C-5 position of the electron-rich heteroarene could be observed using the Pd-catalyzed method. In contrast, a competing aryl bromide group, which was tolerated under our reaction conditions, showed full conversion in the metal-catalyzed protocol. This result highlights the complementary nature of the developed thiolation of heteroarenes to cross-coupling reactions.

While $\mathrm{C}-\mathrm{Cl}$ substitution at the $\mathrm{C}-5$ position for the standard substrate $\mathbf{2 a}$ was observed using the Nickel/photoredox dual catalytic system, this reaction did not require the Nickel catalyst. Moreover, the more demanding substrate $\mathbf{2 l}$ resulted in formation of two undesired products, while the desired product was not observed. 


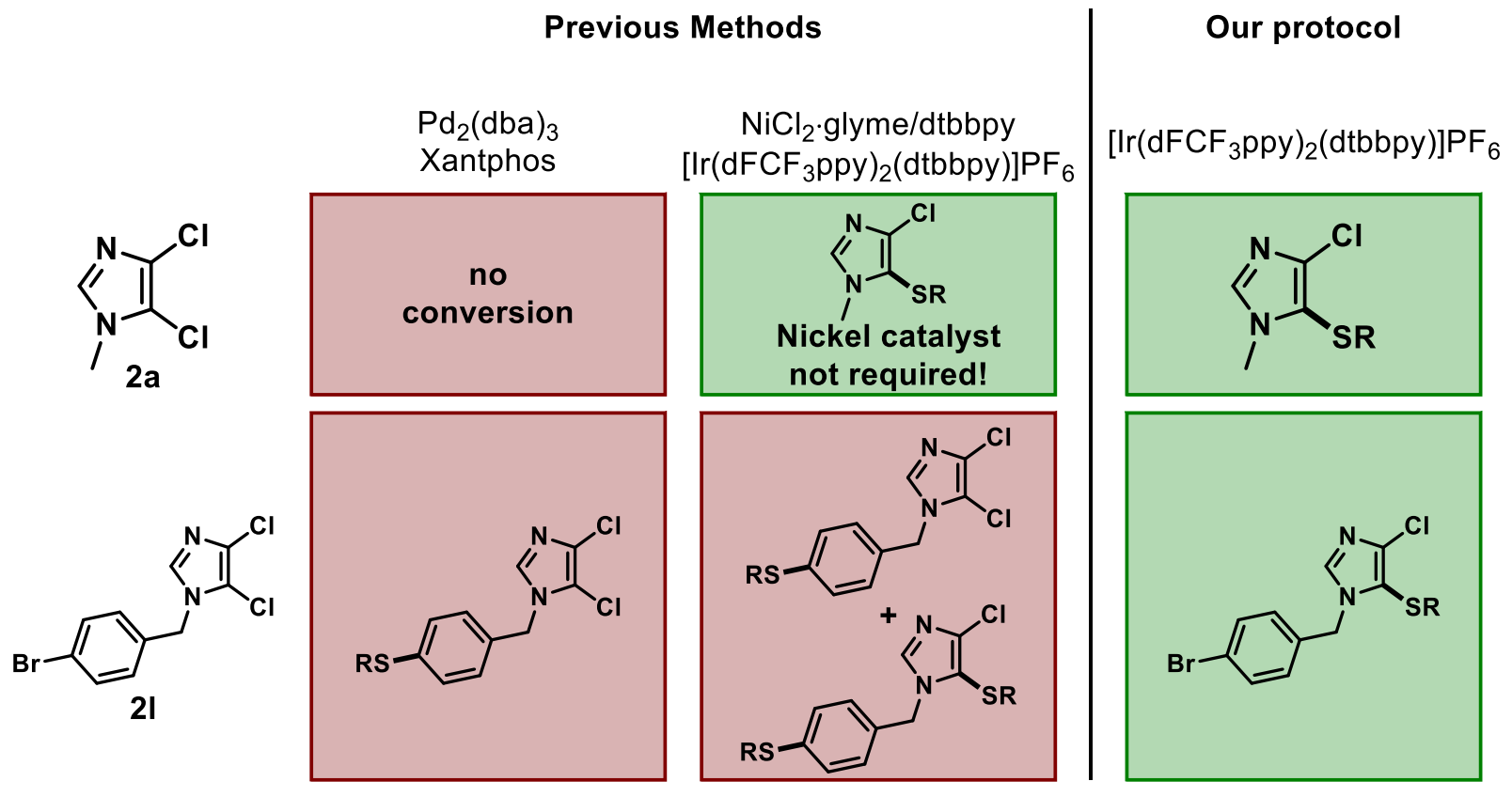

Figure S6. Summary of the comparison of the developed protocol to previous $\mathrm{C}-\mathrm{S}$ coupling reactions. Green boxes indicate formation of the desired C5-substitution products. 


\section{Mechanistic Analysis}

\subsection{Reaction Profile}

A reaction profile was measured in order to get further insight into the time scale of the reaction. 4,5-Dichloro-1-methyl- $1 H$-imidazole $\quad(45.3 \mathrm{mg}, \quad 0.30 \mathrm{mmol}, \quad 1.0$ equiv $)$ and $\left[\operatorname{Ir}\left(\mathrm{dFCF}_{3} \text { ppy }\right)_{2}(\mathrm{dtbbpy}) \mathrm{PF}_{6}(6.7 \mathrm{mg}, 0.006 \mathrm{mmol}, 2.0 \mathrm{~mol} \%)\right.$, were weighed into a Schlenk tube containing a magnetic stirring bar. The tube was evacuated and backfilled with argon three times. DMA (3 mL, 0.1 M) was added, followed by methyl 3-mercaptopropanoate $(49.9 \mu \mathrm{L}$, $0.45 \mathrm{mmol}, 1.5$ equiv) and mesitylene $(41.4 \mu \mathrm{L}, 0.3 \mathrm{mmol}, 1.0$ equiv) as an internal standard. The reaction was stirred under irradiation with $455 \mathrm{~nm}$ LEDs. At selected time points, an aliquot $(100 \mu \mathrm{L})$ was removed from the reaction mixture under a stream of argon and filtered over a plug of silica (elution with EtOAc). The yield was determined via calibrated GC-FID. The determined yield of the product was plotted against the reaction time (see Figure S7).

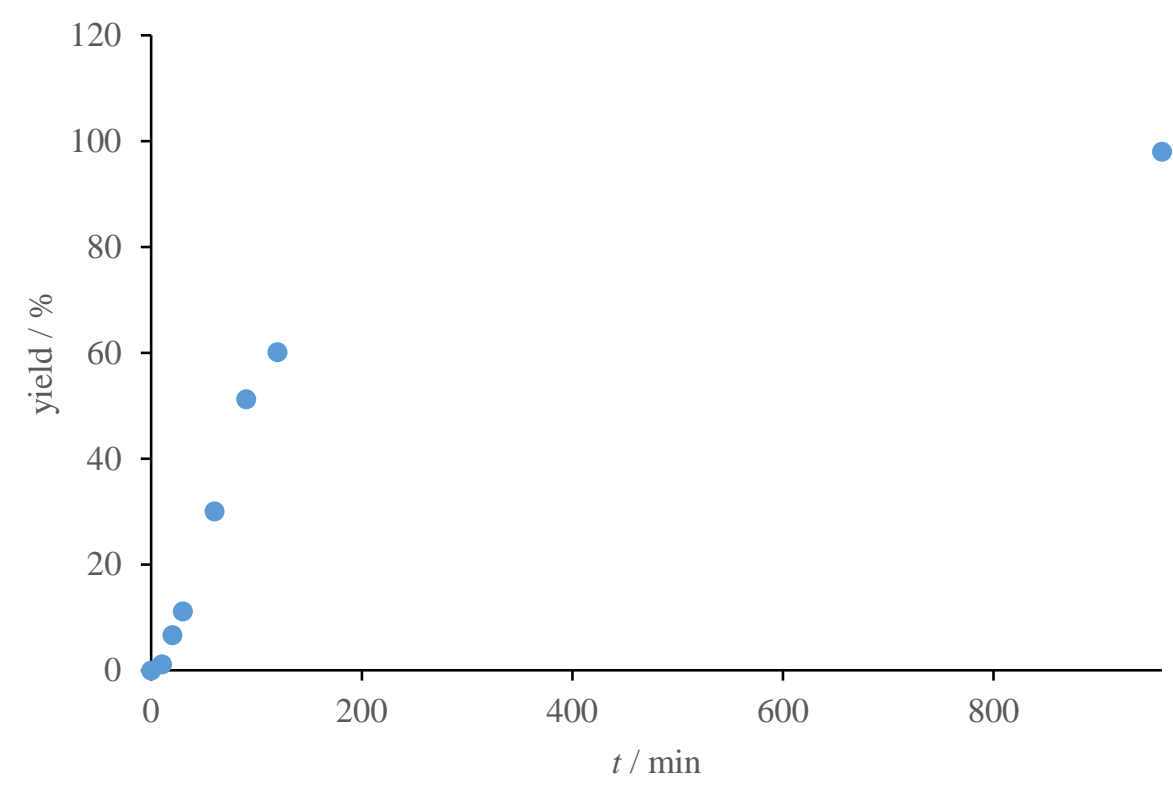

Figure S7. Reaction profile.

The reaction profile shows an induction period between a time of 0 and $10 \mathrm{~min}$. To further investigate this behavior, a second reaction profile was measured. In addition to the conditions mentioned above, $\mathrm{K}_{2} \mathrm{~S}_{2} \mathrm{O}_{8}(1.6 \mathrm{mg}, 0.006 \mathrm{mmol}, 2 \mathrm{~mol} \%$ ) was added to the reaction mixture (1 equiv with respect to the catalyst). A comparison of the two reaction profiles is depicted in Figure S8. The profile with additional $\mathrm{K}_{2} \mathrm{~S}_{2} \mathrm{O}_{8}$ does not show an induction period as observed under the standard conditions. This suggests that the catalyst has to be oxidized first, and a secondary quenching by one of the substrates is part of the reaction mechanism. 


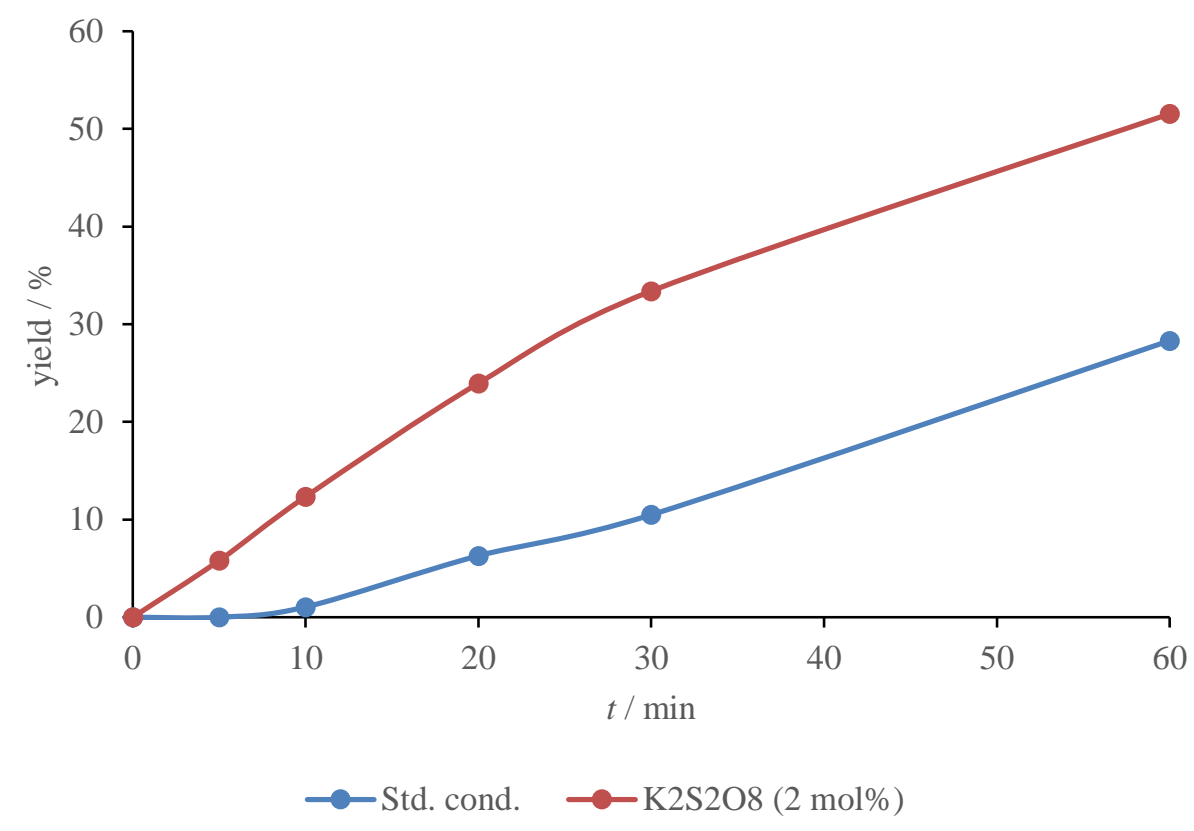

Figure S8. Comparison of the reaction profile under standard conditions and with addition of $\mathrm{K}_{2} \mathrm{~S}_{2} \mathrm{O}_{8}(2 \mathrm{~mol} \%)$.

\subsection{Stern-Volmer Quenching Studies}

All samples used in the luminescence quenching-based screening studies were prepared under oxygen-free conditions. The photocatalysts and potential quenchers were weighed into vials and placed inside a glovebox under an argon atmosphere. DMA was degassed by argon sparging for one hour and placed inside the glovebox. Stern-Volmer luminescence quenching studies were carried out using a $1 \cdot 10^{-5} \mathrm{M}$ solution of $\left[\operatorname{Ir}\left(\mathrm{dFCF}_{3} \text { ppy }\right)_{2}(\mathrm{dtbbpy})\right] \mathrm{PF}_{6}$ and variable concentrations of 4,5-dichloro-1-methyl-1 $\mathrm{H}$-imidazole or methyl 3-mercaptopropanoate in dry DMA at room temperature under an argon atmosphere. The samples were prepared in $1.4 \mathrm{~mL}$ quartz cuvettes, equipped with PTFE stoppers and sealed with parafilm inside an argon filled glovebox. The solutions were irradiated at $420 \mathrm{~nm}$ and the luminescence was measured at $477 \mathrm{~nm}$. The ratio of $I_{0} / I$ was plotted as a function of the quencher concentration (see Figure S9, $\mathrm{I}_{0}=$ emission intensity of the photocatalyst in isolation at the specified wavelength; $\mathrm{I}=$ observed emission intensity of the photocatalyst with added quencher). Each value was measured three times to estimate the error of the method. No quenching could be observed for any of the substrates, supporting the hypothesis of a secondary quenching mechanism. 


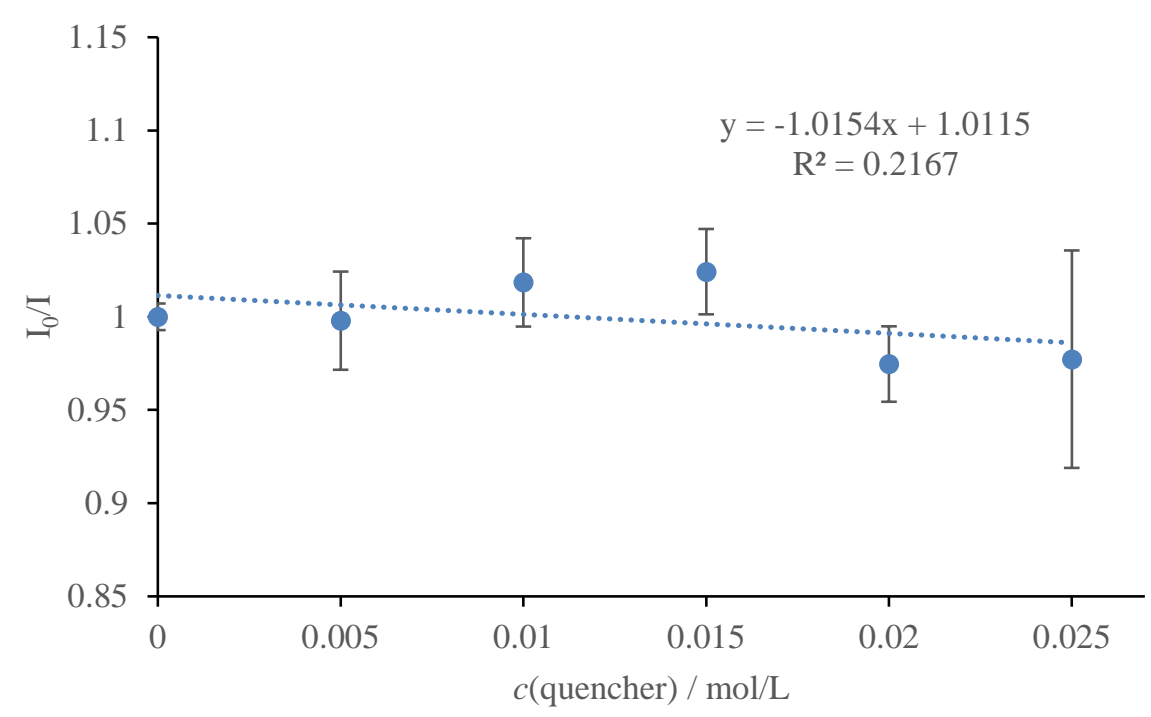

Figure S9. Stern-Volmer quenching study using 4,5-dichloro-1-methyl- $1 H$-imidazole as the quencher. Each value was determined three times and the average value was plotted. Error bars indicate the standard deviation.

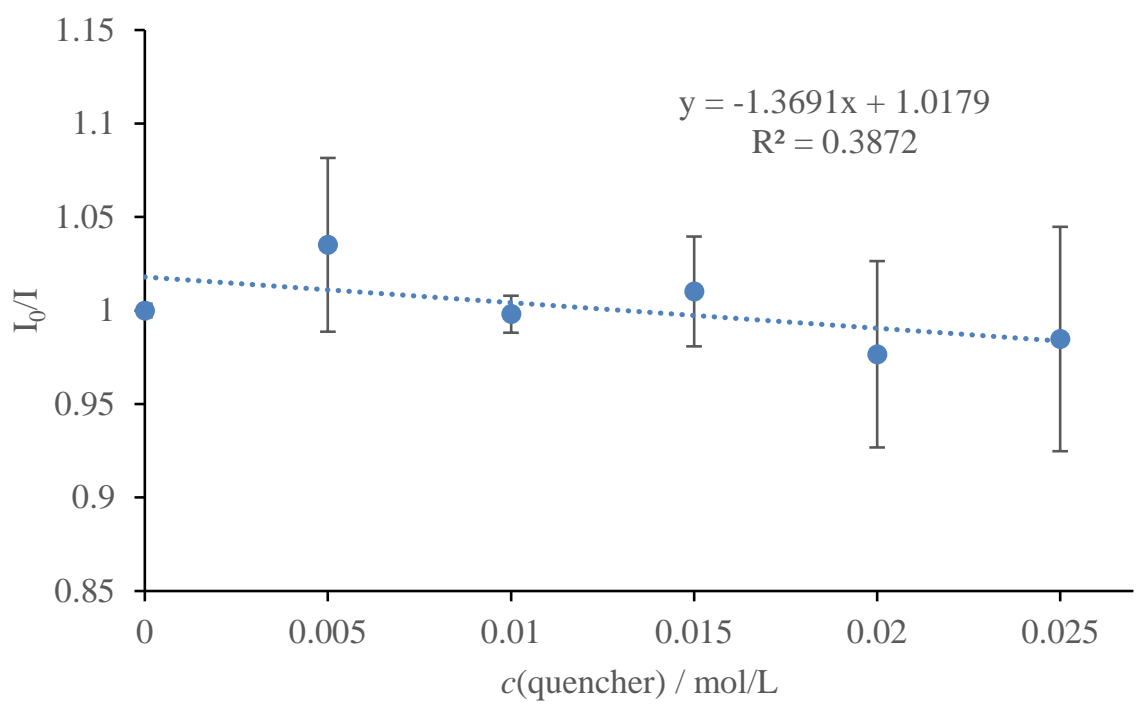

Figure S10. Stern-Volmer quenching study using methyl 3-mercaptopropanoate as the quencher. Each value was determined three times and the average value was plotted. Error bars indicate the standard deviation.

\subsection{Thiol-ene Competition Experiment}

To get further evidence for the formation of thiyl radicals, a quantitative competition experiment was carried out. Therefore, 1-octene was added in a stoichiometric fashion as a scavenger of thiyl radicals via a thiol-ene reaction. 


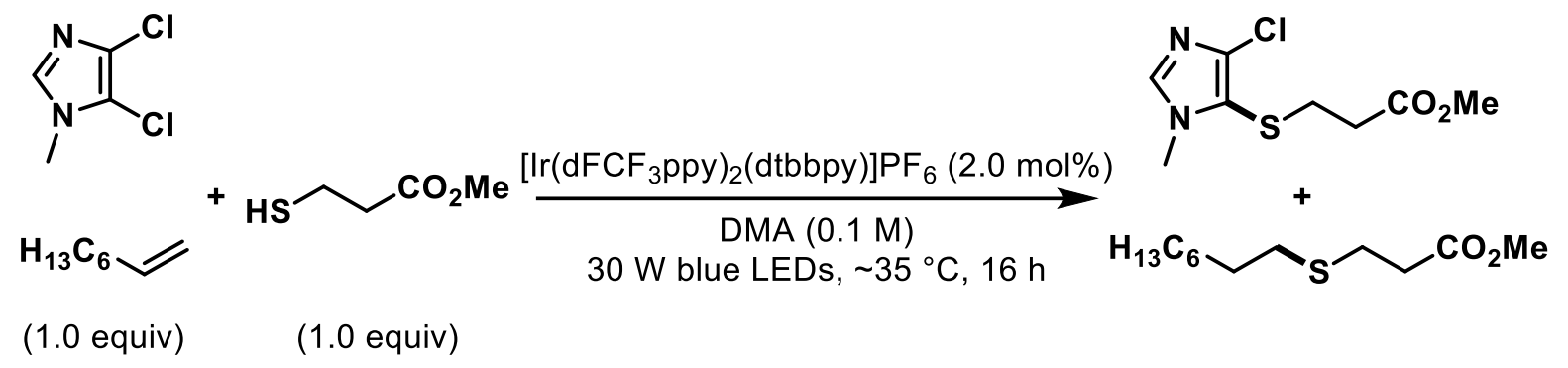

4,5-Dichloro-1-methyl- $1 H$-imidazole $\quad(45.3 \mathrm{mg}, \quad 0.30 \mathrm{mmol}, \quad 1.0$ equiv $) \quad$ and $\left[\operatorname{Ir}\left(\mathrm{dFCF}_{3} \text { ppy }\right)_{2}(\mathrm{dtbbpy}) \mathrm{PF}_{6}(6.7 \mathrm{mg}, 0.006 \mathrm{mmol}, 2.0 \mathrm{~mol} \%)\right.$, were weighed into a Schlenk tube containing a magnetic stirring bar. The tube was evacuated and backfilled with argon three times. DMA (3 mL, 0.1 M) was added, followed by methyl 3-mercaptopropanoate $(33.2 \mu \mathrm{L}$, $0.30 \mathrm{mmol}, 1.0$ equiv) and 1 -octene $(47.4 \mu \mathrm{L}, 0.30 \mathrm{mmol}, 1.0$ equiv). The reaction was stirred under irradiation with $455 \mathrm{~nm}$ LEDs for $16 \mathrm{~h}$ at room temperature. The crude mixture was diluted with EtOAc. $\mathrm{NaHCO}_{3}$-solution (sat., $10 \mathrm{~mL}$ ) and water $(10 \mathrm{~mL})$ were added, the layers were separated, and the organic layer was washed with water $(2 \times 20 \mathrm{~mL})$ and dried over $\mathrm{Na}_{2} \mathrm{SO}_{4}$. The solvent was removed under reduced pressure and the crude product was purified by column chromatography over silica gel (pentane:EtOAc $=20: 1-$ EtOAc) to afford the trapping product via radical thiol-ene (methyl 3-(octylthio)propanoate, $47.2 \mathrm{mg}, 68 \%$, colorless oil) as well as the standard reaction product (methyl 3-((4-chloro-1-methyl-1H-imidazol-5yl)thio)propanoate, $22.3 \mathrm{mg}, 32 \%$ ).

Analytical data for methyl 3-(octylthio)propanoate (8):

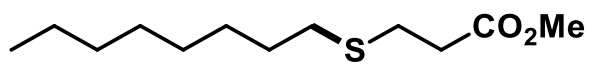

${ }^{1} \mathbf{H}$ NMR (400 MHz, Chloroform-d) $\delta 3.69(\mathrm{~s}, 3 \mathrm{H}), 2.77(\mathrm{t}, J=7.5 \mathrm{~Hz}, 2 \mathrm{H}), 2.60(\mathrm{t}, J=7.2 \mathrm{~Hz}$, $2 \mathrm{H}), 2.51(\mathrm{t}, J=7.3 \mathrm{~Hz}, 2 \mathrm{H}), 1.64-1.49(\mathrm{~m}, 2 \mathrm{H}), 1.40-1.22(\mathrm{~m}, 10 \mathrm{H}), 0.91-0.83(\mathrm{~m}, 3 \mathrm{H})$; ${ }^{13}$ C NMR (101 MHz, Chloroform-d) $\delta 172.6,51.9,34.9,32.3,31.9,29.7,29.3,29.0,27.1,22.8$, 14.2 (one signal overlapping); Rf (pentane:EtOAc = 20:1): 0.30; HR-MS (ESI) m/z calculated for $\left[\mathrm{C}_{12} \mathrm{H}_{24} \mathrm{O}_{2} \mathrm{SNa}\right]^{+}\left([\mathrm{M}+\mathrm{Na}]^{+}\right): 255.1389$, measured: 255.1406 . 


\subsection{Investigation of Traditional Radical Initiators}

In order to investigate the possibility of a radical chain process, the photocatalyst was replaced by AIBN (azobisisobutyronitrile) or DBPO (benzoyl peroxide) as traditional radical initiators and the reaction was heated to $85^{\circ} \mathrm{C}$ instead of irradiation with visible light. The results are given in Table S5. While only traces of product 3a could be obtained using DBPO, significant reactivity was observed using AIBN (29\%). This is a strong indication for a radical chain mechanism.
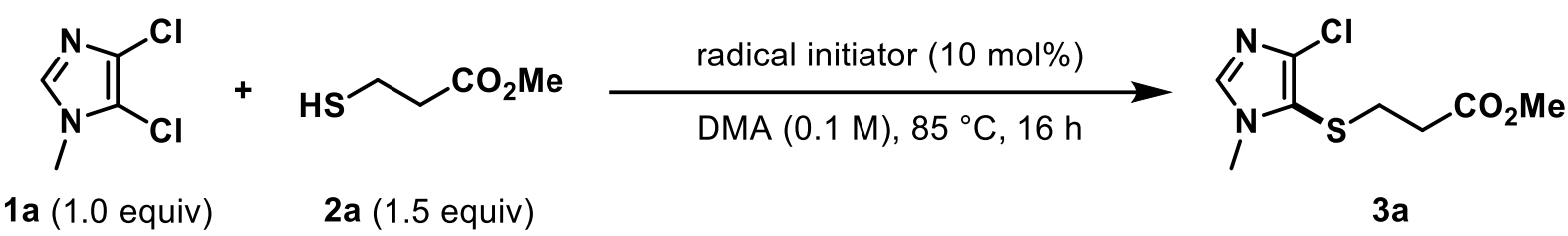

4,5-Dichloro-1-methyl-1H-imidazole (1a, $15.1 \mathrm{mg}, 0.10 \mathrm{mmol}, 1.0$ equiv) and the respective radical initiator $(0.01 \mathrm{mmol}, 10 \mathrm{~mol} \%)$ were weighed into a Schlenk tube containing a magnetic stirring bar. The tube was evacuated and backfilled with argon three times. DMA (1 mL, 0.1 M) was added, followed by methyl 3-mercaptopropanoate (2a, $22.2 \mu \mathrm{L}, 0.20 \mathrm{mmol}, 2.0$ equiv). The reaction was heated to $85^{\circ} \mathrm{C}$ and stirred for $16 \mathrm{~h}$. The reaction was allowed to cool to room temperature, mesitylene $(13.8 \mu \mathrm{L}, 0.1 \mathrm{mmol}, 1.0$ equiv) was added as an internal standard and an aliquot $(100 \mu \mathrm{L})$ of the crude reaction mixture was filtered over a plug of silica (elution with EtOAc). The yield was determined via calibrated GC-FID.

Table S5. Results of the reactions carried out under thermal conditions using traditional radical initiators.

\begin{tabular}{ccc}
\hline Entry & Radical initiator $(10 \mathrm{~mol} \%)$ & yield of 3a / \% \\
\hline 1 & AIBN & 29 \\
2 & DBPO & $<3$ \\
3 & None & 0 \\
\hline
\end{tabular}




\subsection{Quantum Yield Analysis}

\section{Determination of the light intensity at $420 \mathrm{~nm}$ :}

Following a literature procedure of Yoon, ${ }^{49}$ the photon flux of the LED $\left(3 \mathrm{~W}, \lambda_{\max }=420 \mathrm{~nm}\right)$ was determined by standard ferrioxalate actinometry. ${ }^{50}$ A solution of ferrioxalate $(0.15 \mathrm{M})$ was prepared by dissolving potassium ferrioxalate hydrate $(737 \mathrm{mg})$ in aq. $\mathrm{H}_{2} \mathrm{SO}_{4}(0.05 \mathrm{M}, 10 \mathrm{~mL})$. A buffered solution of 1,10-phenanthroline was prepared by dissolving 1,10-phenanthroline $(25.0 \mathrm{mg})$ and sodium acetate $(5.63 \mathrm{~g})$ in aq. $\mathrm{H}_{2} \mathrm{SO}_{4}(0.5 \mathrm{M}, 25 \mathrm{~mL})$. Both solutions were stored in the dark. To determine the photon flux of the LED, the ferrioxalate solution $(1.0 \mathrm{~mL})$ was placed in a $4.0 \mathrm{~mL}$ vial and irradiated for $60 \mathrm{~s}$ at $\lambda_{\max }=420 \mathrm{~nm}$ (see Figure $\mathrm{S} 3$ for the emission spectrum of the LED used). After irradiation, the phenanthroline solution $(175 \mu \mathrm{L})$ was added to the vial and the mixture was stirred in the dark for $1 \mathrm{~h}$ to allow the ferrous ions to be completely coordinated by the phenanthroline. The solution was transferred to a quartz cuvette and the absorption of the solution was measured at $510 \mathrm{~nm}$. A non-irradiated sample was also prepared and the absorption at $510 \mathrm{~nm}$ was measured. Each sample preparation and measurement was repeated two more times. The average of the absorption of the irradiated and non-irradiated samples was determined and used to calculate the conversion applying eq. 1.

$$
n\left(\mathrm{Fe}^{2+}\right)=\frac{V \cdot \Delta A(510 \mathrm{~nm})}{l \cdot \varepsilon}
$$

where $\mathrm{V}$ is the total volume $(0.001175 \mathrm{~L})$ of the solution after addition of phenanthroline, $\Delta A$ is the difference in absorption at $510 \mathrm{~nm}$ between the irradiated and non-irradiated solutions, $l$ is the path length $(1.0 \mathrm{~cm})$, and $\varepsilon$ is the molar absorptivity of the ferrioxalate actinometer at 510 $\mathrm{nm}\left(11100 \mathrm{~L} \mathrm{~mol}^{-1} \mathrm{~cm}^{-1}\right){ }^{50 \mathrm{a}}$ The photon flux $\left(\Phi_{\mathrm{q}}\right)$ can be calculated using eq. 2 ,

$$
\Phi_{\mathrm{q}}=\frac{n\left(\mathrm{Fe}^{2+}\right)}{\Phi_{\mathrm{F}} \cdot t \cdot f}
$$

where $\Phi_{\mathrm{F}}$ is the quantum yield for the ferrioxalate actinometer $\left(1.12\right.$ at $\left.\lambda_{\mathrm{ex}}=420 \mathrm{~nm}\right),{ }^{51} t$ is the irradiation time $(60 \mathrm{~s})$, and $f$ is the fraction of light absorbed at $\lambda_{\mathrm{ex}}=420 \mathrm{~nm}$ by the ferrioxalate actinometer. This value is calculated using eq. 3 where $A(420 \mathrm{~nm})$ is the absorption of the ferrioxalate solution at $420 \mathrm{~nm}$. An absorption spectrum gave an $A(420 \mathrm{~nm})$ value of $>3$, indicating that the fraction of absorbed light $(f)$ is $>0.999$.

$$
f=1-10^{-A(420 \mathrm{~nm})}
$$

The photon flux was thus calculated (average of three experiments) to be $1.12 \cdot 10^{-9}$ einsteins $\mathrm{s}^{-1}$.

\section{Determination of the reaction quantum yield:}

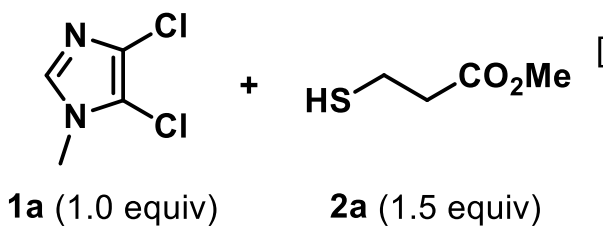
$\left.\underset{\operatorname{Ir}(\mathrm{dFCF}}{3} \mathrm{ppy}_{2}(\mathrm{dtbbpy})\right] \mathrm{PF}_{6} \quad(2.0 \mathrm{~mol} \%)$
DMA $(0.1 \mathrm{M}), 15 \mathrm{~min}$<smiles>CC(=O)CCSc1c(Cl)ncn1C</smiles>
$3 a$

4,5-Dichloro-1-methyl-1H-imidazole (1a, $\quad 15.1 \mathrm{mg}, \quad 0.10 \mathrm{mmol}, \quad 1.0$ equiv) and [Ir(dFCF 3 ppy $)_{2}(\mathrm{dtbbpy}) \mathrm{PF}_{6}(2.2 \mathrm{mg}, 0.002 \mathrm{mmol}, 2.0 \mathrm{~mol} \%)$, were weighed into an ovendried $4.0 \mathrm{~mL}$ screw-cap vial containing a magnetic stirring bar. The tube was evacuated and 
backfilled with argon three times. DMA $(1 \mathrm{~mL}, 0.1 \mathrm{M})$ was added, followed by methyl 3mercaptopropanoate $(\mathbf{2 a}, 22.2 \mu \mathrm{L}, 0.20 \mathrm{mmol}, 2.0$ equiv). The reaction was stirred under irradiation with a single blue $\operatorname{LED}\left(3 \mathrm{~W}, \lambda_{\max }=420 \mathrm{~nm}\right)$ at room temperature for $15 \mathrm{~min}$. After irradiation, the yield was determined by GC-FID analysis using mesitylene as an internal standard. The yield was determined to be $8.4 \%\left(8.4 \cdot 10^{-6} \mathrm{~mol}\right)$. The reaction quantum yield $(\Phi)$ was determined using eq. 4 where the photon flux is $1.12 \cdot 10^{-9}$ einsteins $\mathrm{s}^{-1}$ (determined by actinometry as described above), $t$ is the reaction time $(15 \mathrm{~min})$ and $f_{\mathrm{R}}$ is the fraction of incident light absorbed by the reaction mixture, determined using eq 3 . An absorption spectrum of the reaction mixture gave an absorbance value of $>3$ at $420 \mathrm{~nm}$, indicating that essentially all the incident light $\left(f_{\mathrm{R}}>0.999\right)$ is absorbed by the photocatalyst.

$$
\Phi=\frac{n(\text { product })}{\Phi_{\mathrm{q}} \cdot t \cdot f_{\mathrm{R}}}
$$

The reaction quantum yield $(\Phi)$ was thus determined to be $\Phi=8.4$.

\section{Determination of the reaction quantum yield with addition of $\mathrm{K}_{2} \mathrm{~S}_{2} \mathrm{O}_{8}$ :}

As an induction period is observed under the standard reaction conditions (see Figure S8) and no product formation can be observed after a reaction time of $5 \mathrm{~min}$, we further determined the quantum yield after a shorter irradiation time under addition of $\mathrm{K}_{2} \mathrm{~S}_{2} \mathrm{O}_{8}(2.0 \mathrm{~mol} \%)$ to get a more accurate estimation of the actual quantum yield.

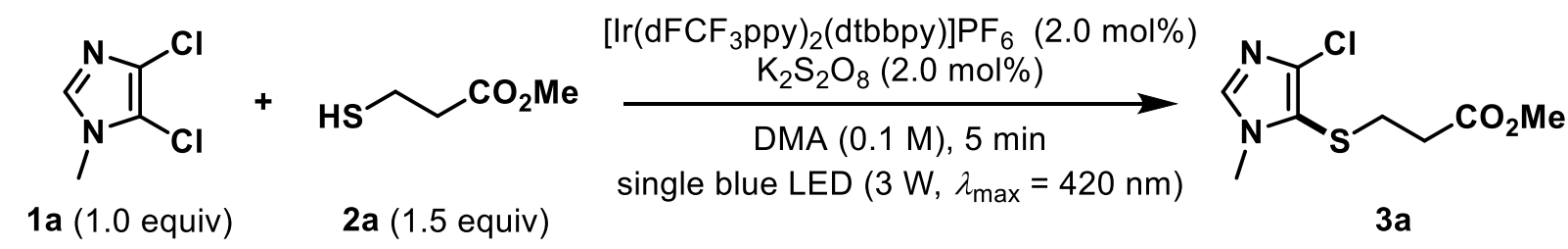

4,5-Dichloro-1-methyl-1H-imidazole (1a, $15.1 \mathrm{mg}, 0.10 \mathrm{mmol}, 1.0$ equiv), $\mathrm{K}_{2} \mathrm{~S}_{2} \mathrm{O}_{8}(0.5 \mathrm{mg}$, $0.002 \mathrm{mmol}, 2.0 \mathrm{~mol} \%)$ and $\left[\operatorname{Ir}\left(\mathrm{dFCF}_{3} \mathrm{ppy}\right)_{2}(\mathrm{dtbbpy})\right] \mathrm{PF}_{6}(2.2 \mathrm{mg}, 0.002 \mathrm{mmol}, 2.0 \mathrm{~mol} \%)$, were weighed into an oven-dried $4.0 \mathrm{~mL}$ screw-cap vial containing a magnetic stirring bar. The tube was evacuated and backfilled with argon three times. DMA (1 mL, $0.1 \mathrm{M})$ was added, followed by methyl 3-mercaptopropanoate (2a, $22.2 \mu \mathrm{L}, 0.20 \mathrm{mmol}, 2.0$ equiv). The reaction was stirred under irradiation with a single blue LED $\left(3 \mathrm{~W}, \lambda_{\max }=420 \mathrm{~nm}\right)$ at room temperature for $5 \mathrm{~min}$. After irradiation, the yield was determined by GC-FID analysis using mesitylene as an internal standard. The yield was determined to be $4.1 \%\left(4.1 \cdot 10^{-6} \mathrm{~mol}\right)$. The reaction quantum yield $(\Phi)$ was determined using eq. 4 where the photon flux is $1.12 \cdot 10^{-9}$ einsteins $\mathrm{s}^{-1}$ (determined by actinometry as described above), $t$ is the reaction time ( $5 \mathrm{~min}$ ) and $f_{\mathrm{R}}$ is the fraction of incident light absorbed by the reaction mixture, determined using eq 3 . An absorption spectrum of the reaction mixture gave an absorbance value of > 3 at $420 \mathrm{~nm}$, indicating that essentially all the incident light $\left(f_{\mathrm{R}}>0.999\right)$ is absorbed by the photocatalyst.

$$
\Phi=\frac{n(\text { product })}{\Phi_{\mathrm{q}} \cdot t \cdot f_{\mathrm{R}}}
$$

The reaction quantum yield $(\Phi)$ was thus determined to be $\Phi=12.3$. 


\subsection{Computational Investigation of the Reaction Mechanism}

All computations were carried out using the ORCA 4.1.1 software package. ${ }^{52}$ The geometries of all relevant structures were optimized using Handy's range-separated CAM-B3LYP functional $^{53}$ and Grimme's D3 dispersion correction $^{54}$ in the def2-TZVPP basis set, as developed by Ahlrichs and co-workers, ${ }^{55}$ with the CPCM continuum solvation model ${ }^{56}$ (presets for DMF). No internal coordinate or symmetry constraints were applied. Optimized geometries were verified to be local minima on the respective potential energy landscape by the absence of negative eigenvalues of the Hessian, as obtained from a harmonic frequency calculation at the same level. Transition states were characterized by one negative eigenvalue of the Hesse matrix and the respective eigenvector vibration.

Free enthalpies were calculated from the electronic energy at the stationary point, corrected by zero-point vibrational energy (ZPVE), thermal, enthalpy and entropy corrections, as obtained from the harmonic frequency calculation (see Table S6). All geometries were visualized using Jmol. ${ }^{57}$

The free enthalpy profile of the reaction of the standard substrate 4,5-dichloro-1-methyl-1Himidazole with methanethiol supports the hypothesis of a radical chain mechanism (see Figure $\mathrm{S} 11$, all energies relative to $\mathbf{1 a}$ and $\mathbf{A})$. As a first step, substitution of the $\mathrm{C}-\mathrm{Cl}$ bond with a thiyl radical at the $\mathrm{C} 5$-position proceeds via a concerted transition state $\mathbf{B}\left(\Delta G^{\ddagger}=15.3 \mathrm{kcal} / \mathrm{mol}\right)$ to give a $\Pi$-complex-type intermediate $(\Delta G=-1.2 \mathrm{kcal} / \mathrm{mol})$. Rotation around the $\mathrm{C}-\mathrm{S}$ bond via a low-barrier transition state $\mathbf{T S}_{\text {rot }}\left(\Delta G^{\ddagger}=-0.2 \mathrm{kcal} / \mathrm{mol}\right)$ results in formation of the radical cation of the product $\mathbf{C}$ and a chloride anion (exergonic, $\Delta G=-7.7 \mathrm{kcal} / \mathrm{mol}$ ). That each transition state leads to the respective intermediates was confirmed via following the intrinsic reaction coordinate. In contrast, formation of a chlorine radical and the (neutral) product would be significantly endergonic $(\Delta G=+10.4 \mathrm{kcal} / \mathrm{mol})$. Attempts to find a stable oxidized Meisenheimer-type intermediate (6-complex) were unsuccessful and analysis of the potential energy surface clearly suggests a concerted transition state. Substitution at the C5-position is kinetically favored over C4-substitution by $\Delta \Delta G^{\ddagger}=4.6 \mathrm{kcal} / \mathrm{mol}$, rationalizing the observation of a single product. The radical chain is propagated by regeneration of a thiyl radical via an oxidative proton-coupled electron transfer (PCET, $\Delta G=-9.7 \mathrm{kcal} / \mathrm{mol}$ ). To estimate the transition state energy for this step, an upper limit approximation was carried out. The threecomponent transition state energy is supposed to be lower than the energy of the intermediates generated via either first executing the proton transfer $(\Delta G=20.8 \mathrm{kcal} / \mathrm{mol})$ or the electron transfer $(\Delta G=11.5 \mathrm{kcal} / \mathrm{mol})$, respectively. ${ }^{58}$ Thus, the transition state energy should be lower than $11.5 \mathrm{kcal} / \mathrm{mol}$ and the radical substitution is the rate-determining step of the reaction. 


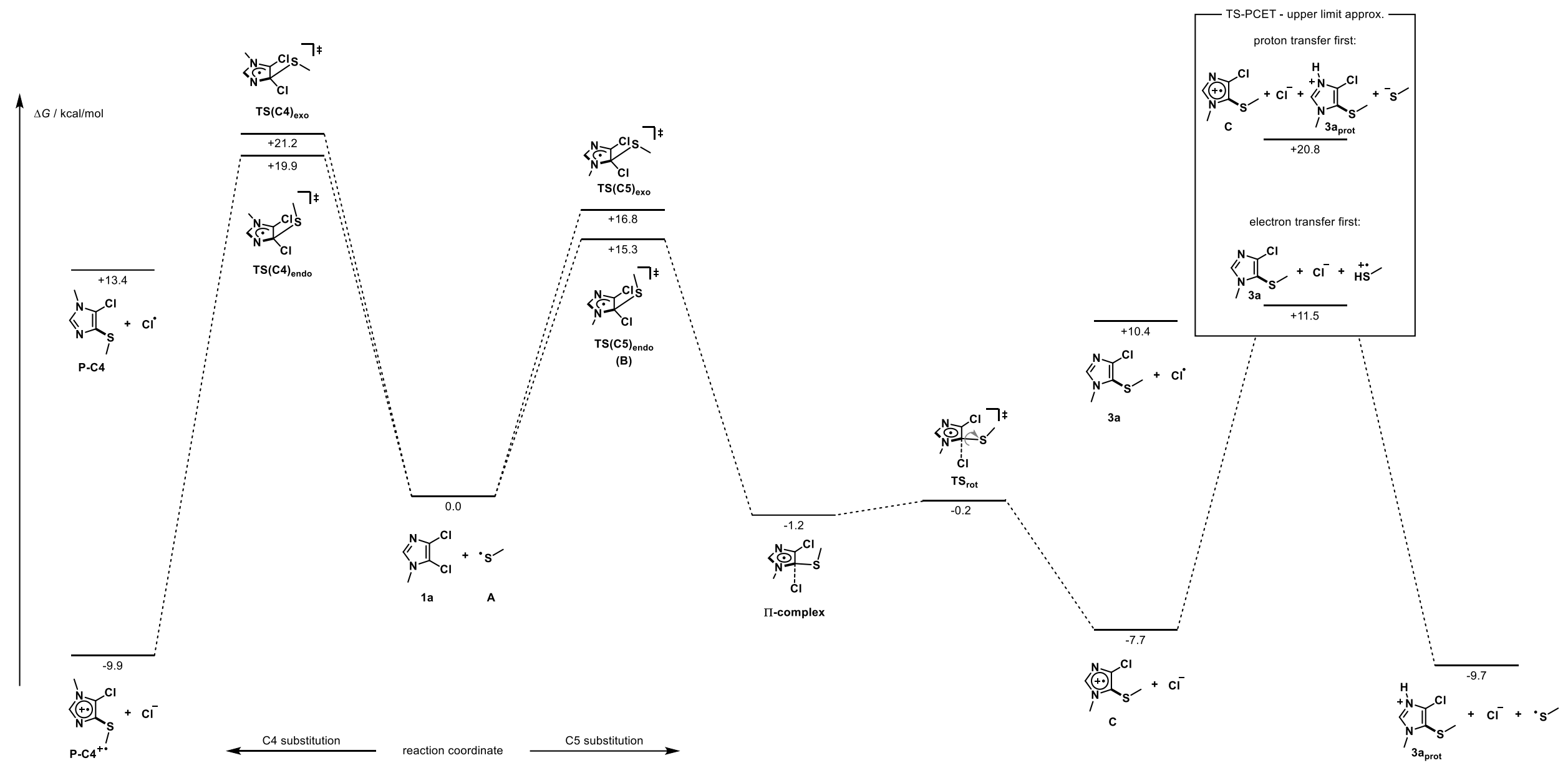

Figure S11. Free Enthalpy (Gibbs free energy) profile comparing radical substitution at the C4 and C5 positions. For the transition state (TS) of the proton-coupled energy transfer (PCET), an upper limit approximation was performed. 
Table S6. Stationary point energies of relevant structures. All energies are given in Hartree $\left(E_{\mathrm{h}}\right)$.

\begin{tabular}{|c|c|c|c|c|c|c|}
\hline & $\begin{array}{c}\text { Electronic } \\
\text { Energy / } E_{\mathrm{h}}\end{array}$ & $\mathrm{ZPVE} / E_{\mathrm{h}}$ & $\begin{array}{l}\text { Thermal } \\
\text { Corr. / } E_{\mathrm{h}}\end{array}$ & $\begin{array}{l}\text { Enthalpy } \\
\text { Corr. } / E_{\mathrm{h}}\end{array}$ & $\begin{array}{c}\text { Entropy } \\
\text { Corr. / } E_{\mathrm{h}}\end{array}$ & $\begin{array}{c}\text { Free } \\
\text { Enthalpy / } E_{\mathrm{h}}\end{array}$ \\
\hline $1 \mathbf{a}$ & -1184.76909 & 0.08157442 & 0.00742613 & 0.00094421 & -0.04123613 & -1184.72038 \\
\hline MeSH & -438.720754 & 0.04626157 & 0.00359221 & 0.00094421 & -0.02874318 & -438.698699 \\
\hline $\operatorname{MeS}^{\bullet}(\mathbf{A})$ & -438.077504 & 0.03659913 & 0.00306261 & 0.00094421 & -0.02821688 & -438.065115 \\
\hline $\mathrm{MeS}^{-}$ & -438.239054 & 0.03676462 & 0.00304199 & 0.00094421 & -0.02757774 & -438.225881 \\
\hline $\mathrm{MeSH}^{+}$ & -438.479555 & 0.04555776 & 0.00354839 & 0.00094421 & -0.02923083 & -438.458736 \\
\hline $\mathrm{Cl}^{-}$ & -460.406289 & 0.00000002 & 0.00141627 & 0.00094421 & -0.01740262 & -460.421331 \\
\hline $\mathrm{Cl}^{*}$ & -460.167418 & 0.00000002 & 0.00141627 & 0.00094421 & -0.01805708 & -460.183114 \\
\hline $3 \mathbf{a}$ & -1162.67049 & 0.11994431 & 0.00957883 & 0.00094421 & -0.04572404 & -1162.58575 \\
\hline $\mathbf{3 a}^{+\cdot}(\mathbf{C})$ & -1162.46114 & 0.12008074 & 0.0093251 & 0.00094421 & -0.04561548 & -1162.37641 \\
\hline 3aprot & -1163.11531 & 0.13726774 & 0.00945952 & 0.00094421 & -0.04557656 & -1163.01321 \\
\hline P-C4 & -1162.66588 & 0.11998919 & 0.00955375 & 0.00094421 & -0.04565651 & -1162.58105 \\
\hline $\mathrm{P}-\mathrm{C4}^{+{ }^{+}}$ & -1162.46375 & 0.11974223 & 0.00964122 & 0.00094421 & -0.04654499 & -1162.37997 \\
\hline $\begin{array}{l}\text { TS }(\text { C5 } 5)_{\text {endo }} \\
\text { (B) }\end{array}$ & -1622.84178 & 0.11891288 & 0.01122221 & 0.00094421 & -0.05034056 & -1622.76104 \\
\hline TS(C5) $)_{\text {exo }}$ & -1622.83925 & 0.1186788 & 0.01125852 & 0.00094421 & -0.05038945 & -1622.75876 \\
\hline TS(C4)endo & -1622.83466 & 0.11888865 & 0.01119041 & 0.00094421 & -0.05014968 & -1622.75378 \\
\hline TS $(\mathbf{C 4})_{\text {exo }}$ & -1622.83236 & 0.11875204 & 0.01128546 & 0.00094421 & -0.05039233 & -1622.75177 \\
\hline П-complex & -1622.86896 & 0.12014965 & 0.01164242 & 0.00094421 & -0.05110071 & -1622.78733 \\
\hline TS & -1622.86758 & 0.11981646 & 0.01103388 & 0.00094421 & -0.04994712 & -1622.78573 \\
\hline
\end{tabular}


$1 \mathbf{a}$

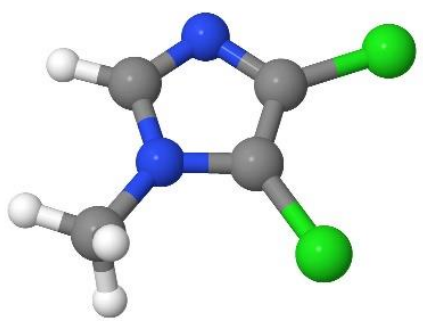
C 8.33650762251332
$-2.04137657158171$
0.11172478040645
C $8.45558784047116 \quad-3.39815474607821$
0.06465995602425
N $7.36985680431518 \quad-3.94769788050061$
$-0.53146281430053$
C 6.59673607348852
$-2.93168275150619$
$-0.84419151281887$
N 7.13451162257506
$-1.75095858106235$
$-0.47697938557345$
C 6.55673584085953
$-0.43467096482593$
$-0.66756668012683$
H 5.59998937573441
$-0.55581765906452$
$-1.16477969058219$
H 7.21051961055076
$0.17526070754274 \quad-1.28552787001006$
H 6.40598529654243
0.05099940164281
0.29305542314408
Н 5.64065190624884
$-2.99417610817893$
$-1.33605135861742$
Cl 9.37222969669700
$-0.84919206112548$
0.73333672726185
Cl 9.75910831000378
$-4.33801278526161$
0.65281242519270

\section{MeSH}

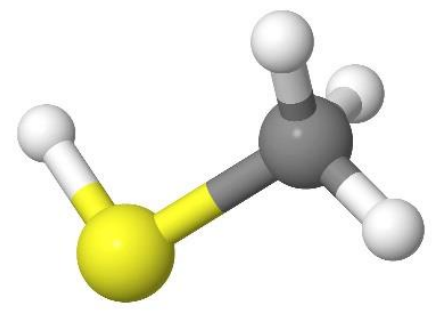
C -6.10374846499389
$-0.61684789849442$
0.00053858468568
S -4.29242640143883
$-0.60568254014942$
$-0.02349117405882$
H $\quad-6.50164547151636$
$-1.16264648887159$
$-0.84930501626948$
H $\quad-6.49930680694968$
0.39395297291623
0.01668203823134
H $\quad-6.39435952392598$
$-1.12556486097865$
0.91586962125022
H $\quad-4.12783333117525$
0.04698881557785
$-1.17982405383894$
C -6.07959293738973
$-0.61356373353718$
$-0.00402406774083$
$\begin{array}{lll}\text { S } & -4.29507059423563 & -0.63417725760348\end{array}$
0.03295359021711
H $\quad-6.46709668878206-1.16357060345040$
$-0.85745777417775$
H $\quad-6.46700687556068 \quad 0.40156553508416$
0.01638408769029

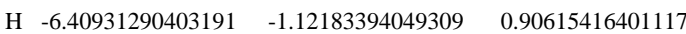

\section{$\mathrm{MeS}^{-}$}

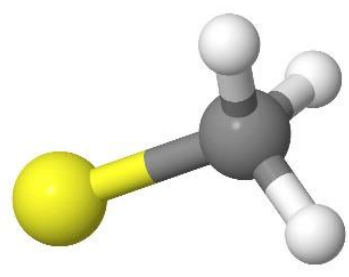

$\begin{array}{llll}\text { C } & -6.06997159560758 & -0.62674893348694 & 0.01946956910145 \\ \text { S } & -4.23948767544253 & -0.62077303239440 & 0.00908447375956 \\ \text { H } & -6.47296673256934 & -1.14795786014877 & -0.84948946140007 \\ \text { H } & -6.47272255753789 & 0.38656689031425 & 0.00728126814878 \\ \text { H } & -6.46293143884266 & -1.12266706428414 & 0.90766415039028\end{array}$

\section{$\mathrm{MeSH}^{+\bullet}$}

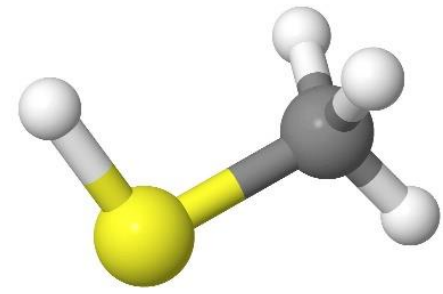
C -6.09638149737652
$-0.63095695495395$
0.02629521812990
S $-4.34103114106809 \quad-0.61940893034489$
$-0.02493749685069$
$\begin{array}{lll}\text { H } & -6.45357032897892 & -1.15230530191088\end{array}$
$-0.86546598489539$
H $\quad-6.44622014026979 \quad 0.40267847989871$
$-0.01439735912373$
H $\quad-6.40394560583657-1.13186093427533$
0.93689939534894
H $\quad-4.17817128647010 \quad 0.06205364158634$
$-1.17792377260903$

3a

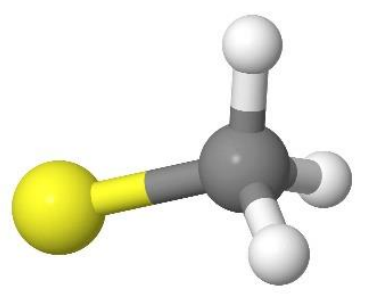



$\begin{array}{lll}\text { C } 8.22146113049360 & -3.21263214190572\end{array}$
N $7.00239438215701 \quad-3.73222748009699$
0.31148545678064
C 6.34709830195772
$-2.76097889720933$
0.05250965352231
N $7.09259597636130 \quad-1.65517724067178$
$-0.55354665871601$
C 6.67629550391369
$-0.41553467663827$
H 5.65726598214618
$-0.53900016802076$
H 7.32468609864164
$-0.18404448270311$
0.40043726487455
H 6.71108471293224
$-2.81844572109332$
H 5.33171699838630
S 9.65459301986833
$-0.80234167217045$
Cl 9.43452876778332
$-4.12449363365060$
C 9.32108543622351
0.11347850334168
H 10.13185036053842
0.83071370342476
$-0.56026406321875$
H 9.31979294220578
0.64600025340975
H 8.37682838434255
$-0.69127842524277$
$-1.31680061642566$
$-1.66954846724280$
$-2.15721942018180$
$-0.59965303242368$
$-0.90927438282554$
$-0.09650997899241$
1.11005244004949
1.43039709833737
1.53564660409528
2.28194017099809
1.36569428684247

\section{$3 \mathbf{a}^{+\bullet}(\mathbf{C})$}

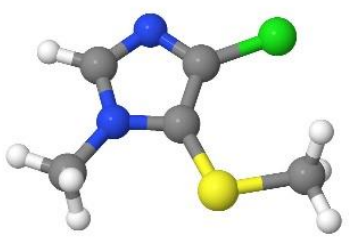
C 7.84515624163016
$-1.44924922671619$
0.12324560519434
C $7.78672958445689-2.70033869450847$
0.82662267273198
N $6.87927291426927 \quad-3.49397578692251$
0.28499877274776
C $6.36057413190089-2.80714530581442$
$-0.72815962167027$
N $6.90441406535843-1.58883515510060$
$-0.86129067593503$
C 6.56177756676366
$-0.59534263140820$
$-1.86810577747474$
H 5.77628645000162
$-1.00724123664015$
$-2.49151815226812$
H 7.43542297822408
$-0.37653175142403 \quad-2.47652655130251$
H $\quad 6.20631804859223$
0.30921549264004
$-1.38155536087090$
H 5.58664121126987
$-3.16422193663850$
$-1.38781290640546$
S $\quad 8.71987843894127 \quad-0.02392605348342 \quad 0.23729618276532$
Cl $8.70268910189474 \quad-3.17196248594262 \quad 2.15461507155111$
C $9.82452715128689-0.21071924852600 \quad 1.63412480601083$
H $\quad 10.35523320980116 \quad 0.73653589762970 \quad 1.66700921547986$
$\begin{array}{llll}\text { H } & 10.52170708866215 & -1.02292905407908 & 1.46109650519574\end{array}$
H $\quad 9.25889181694671 \quad-0.35278282306552 \quad 2.54827021425006$

\section{3aprot}

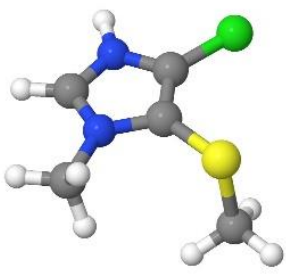
C $8.34045435965742-1.95281190805182$
$-0.08080606461935$
$\begin{array}{llll}\text { C } & 8.26257513387966 & -3.23496256432357 & 0.36791082150425\end{array}$
$\begin{array}{llll}\text { N } & 7.00852531438601 & -3.69812239120583 & 0.08634263160945\end{array}$
C $6.32011941078616 \quad-2.73762194769691 \quad-0.51954292679583$
$\begin{array}{llll}\text { N } & 7.09315895949615 & -1.67817636628679 & -0.63725882090252\end{array}$
C $\quad 6.70256643614573 \quad-0.42143338125077 \quad-1.26084488067623$
H $5.69034261899898 \quad-0.52991429314136 \quad-1.63415682739035$
H $7.37989419285520 \quad-0.20306104370929 \quad-2.07980019094907$
$\begin{array}{llll}\text { H } & 6.73938846169379 & 0.37653030413196 & -0.52600359150882\end{array}$
H $\quad 5.30253288349254 \quad-2.81883612765570 \quad-0.85763223507886$
$\begin{array}{llll}\text { S } & 9.67714896440847 & -0.84936435874232 & -0.06591660408443\end{array}$
Cl $9.41814912444808 \quad-4.18550910006711 \quad 1.14383073027714$
$\begin{array}{llll}\text { C } & 9.30987101080689 & 0.12153102390812 & 1.41848889205573\end{array}$
H $\quad 6.65338244847944 \quad-4.62035607605758 \quad 0.29635655693568$
$\begin{array}{llll}\text { H } & 10.12922667533928 & 0.83006935015780 & 1.51172969155435\end{array}$
H $\quad 9.28180845808508 \quad-0.52098959514483 \quad 2.29274185038780$
H $8.37623554704110 \quad 0.66448847513599 \quad 1.30926096768105$

\section{P-C4}

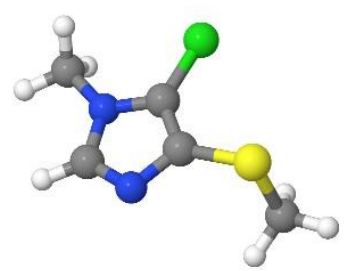
C 8.29742409752311
$-2.03667264384875$
$-0.03433845076852$
C $8.18172549335853-3.31606056328102$
0.43701430585148
N $6.91961523449188 \quad-3.74225017185837$
0.14071242915759
C $6.31550266300673 \quad-2.71007302241773$
$-0.49299893003976$
N $7.10781102869601 \quad-1.67657097673861$
$-0.61914707039559$
H $\quad 5.29794965757497 \quad-2.77845546489454$
$-0.84127439320609$
S $9.69949965404169-1.00048251766078$
Cl 9.29531327787607
$-4.30631301938014$
1.25627899572273
C 9.27193663029542
0.07272046891831
1.42652262493477
$\begin{array}{llll}\text { H } & 10.09328816499423 & 0.77716110312029 & 1.53637840319987\end{array}$
H 9.16946075396796
$-0.50825198178808 \quad 2.33879119304739$ 

H 8.35551425948244
0.61808907361112
1.22047307664852
$-5.03675548783839$
0.43934671504180
H 5.32228515170330
$-5.04601170871876$
0.06034029473807
H $6.32502178517229-5.20382245137037$
1.51329510654353
H 6.91068840864102
$-5.82514063585417$
$-0.04349535399268$

\section{P-C4 ${ }^{+\cdot}$}

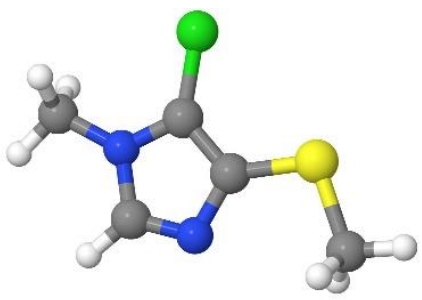
H $\quad 6.66677727855368$
$-1.08964054337671$
$-2.03148525091540$
H 7.76940428343206
$-0.15905382842963$
H 6.16705694110813
$-0.63751284021384$
$-0.99205846357885$
H $7.43687000826137-3.40013177145386$
$-0.39139291259578$
Cl $7.52163993886834 \quad-1.56769675407066$
.25751037167260
Cl $9.25485224077183 \quad-4.57957661965398$
09723622398772
S $10.17637069359142 \quad-1.22847303216305$
C 10.78503672149025
H 11.74371384051325
H $\quad 10.11498517216810 \quad-1.71926179155527 \quad-1.78509643673050$
H $\quad 10.94383005541289-3.00383203569134 \quad-0.87062956280157$

\section{TS(C5) exo}

\begin{abstract}
C $7.66807748513054 \quad-1.45782336259329 \quad 0.12540207831491$
$\begin{array}{llll}\text { C } & 7.77317337698161 & -2.72525635307689 & 0.76477671947221\end{array}$

$\begin{array}{llll}\text { N } & 6.88890284210209 & -3.52155827000789 & 0.15787143399223\end{array}$

$\begin{array}{llll}\text { C } & 6.27842538749723 & -2.76060220391555 & -0.80870181378429\end{array}$

N $6.72185507646993 \quad-1.53593308199039 \quad-0.84770947672158$

H $\quad 5.51574016422445 \quad-3.17831899108964 \quad-1.44582625420354$

$\begin{array}{llll}\text { S } & 8.58130153726907 & -0.11393630358579 & 0.53886453844426\end{array}$

Cl $8.76203659621884 \quad-3.20625761770231 \quad 2.01818995570782$

$\begin{array}{llll}\text { C } & 7.97338389549591 & 1.12020311960033 & -0.60536916919198\end{array}$

H $\quad 6.91185714608808 \quad 1.27531754940738 \quad-0.44011498452307$

H $8.16191812048279 \quad 0.79553967571865 \quad-1.62384706368045$

$\begin{array}{llll}\text { H } & 8.53187964424475 & 2.02366827339078 & -0.38142561736801\end{array}$

$\begin{array}{llll}\text { C } & 6.61887932692097 & -4.92018614160747 & 0.45639704913901\end{array}$

H $\quad 5.83810433249388 \quad-5.25970471681320 \quad-0.21466394900130$

H $\quad 6.28648172109954 \quad-5.01305708684460 \quad 1.48620779884269$

H $7.52065334728031 \quad-5.50494448889011 \quad 0.29946875456109$
\end{abstract}

\section{TS(C5)endo (B)}

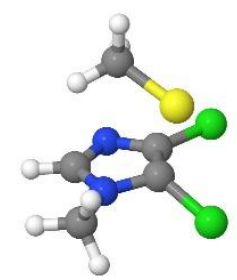
C 8.17654893715042
$-2.36209045650350$
0.70654998209758
C 8.54790188389449
$-3.72941053221894$
N 8.33019115335313
$-4.26556179452140$
C 7.74181682081017
$-3.31144249259389$
$-2.17902712298921$
N 7.56744415816689
$-0.93524475527109$
0.68086670050536
$-0.52161837049324$
$-1.22803200185359$
$-0.52846384312247$
$-1.01298044511917$

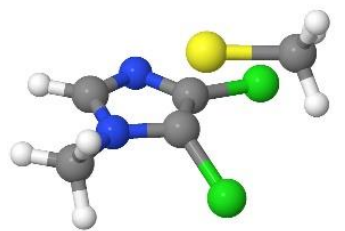

C $8.16807057116660 \quad-2.22400907831709 \quad 0.64385103551479$

$\begin{array}{llll}\text { C } & 8.56749900728919 & -3.58559937167322 & 0.51493099750403\end{array}$

N $8.20402062168438 \quad-4.08367368796791 \quad-0.66937149994968$

C $7.48579938689483 \quad-3.13153388066978 \quad-1.24238760106516$

N $7.35708892310785 \quad-2.03711435154309 \quad-0.47309848832467$

C $6.68028487165023 \quad-0.80574547087959 \quad-0.81728768772894$

H $6.19577325776905 \quad-0.94055900216367 \quad-1.77871801343215$

H $7.40081504181512 \quad 0.00745322390814 \quad-0.88039301971154$

H $5.93195219927122 \quad-0.56883812910418 \quad-0.06543622076400$

H $7.03115033146770 \quad-3.20041859801823 \quad-2.21684585662416$

Cl $7.67351611772798 \quad-1.53036932908061 \quad 2.15385945128322$

Cl $9.46469987489701 \quad-4.46117509919219 \quad 1.65639357198649$

$\begin{array}{llll}\text { S } & 10.00904304614203 & -1.05391165740252 & 0.07275319125329\end{array}$

C $\quad 10.99203456792494 \quad-1.20506883696032 \quad 1.57246377092039$

$\begin{array}{llll}\text { H } & 11.83111647624259 & -0.51859457986617 & 1.46693111103697\end{array}$

$\begin{array}{llll}\text { H } & 11.37860633406329 & -2.21167411093214 & 1.70232827743364\end{array}$

H $\quad 10.41666937088580 \quad-0.91186804013745 \quad 2.44693698066746$ 


\section{TS(C4)endo}

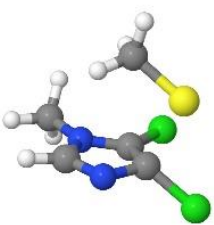
C 8.18067405678161
$-2.34760445690373$
0.65041801053812
C 8.58235654403542
$-3.70795254668612$
0.66744035462189
N 8.33050026867199
$-4.19919059362117$
C 7.69121510286000
$-3.20683216338255$
N 7.53004690256465
$-2.12413670173730$
H $\quad 7.36047417387350$
$-3.35611374591420$
Cl 7.51568424169701
$-1.59882801667168$
Cl 9.35784311054281
$-4.58155024638890$
S $\quad 10.08447278298674$
$-1.19962157682119$
C $\quad 10.79620434343779$
$-1.989743589910279$
H 11.69322001904839
$-1.43068624672953$
H $\quad 10.11293825611333$
$-1.94643064063089$
H 11.08130308886319
$-3.02039847548442$
C 8.67870322737797
$-5.51287376408570$
$-5.60646403484601$
$-6.28123854588441$
H 8.24982664409569
H 9.75951872973104
$-5.62809465510940$
$-0.57202082612846$
$-1.25464354015774$
$-0.55748964169597$
$-2.26991932777436$
2.08500963453846
1.87536422001069
0.54953131925827
$-0.89904490318280$
$-1.16037252458272$
$-1.74494294250198$
$-0.70000411206457$
$-1.07727704413345$
$-2.07941865965496$
$-0.43968909176440$
$-1.11139092532606$

\section{$\operatorname{TS}(\mathrm{C} 4)_{\text {exo }}$}

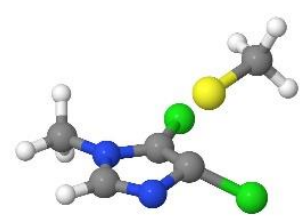
C 8.16320837108366
$-2.22760645943962$
0.59997038596997
C 8.60769084516709
$-3.57798700588819$
0.54536502280500
N 8.26319212771064
$-4.04460081711011$
$-0.68378759881418$
C 7.51131854409746
$-3.06965656644324$
$-1.26525841500321$
N 7.36426678651985
$-2.01478527356894$
$-0.52522733706993$
H $7.08315222755870 \quad-3.21202266835270$
$-2.24481243268857$
Cl $7.62118559951214 \quad-1.55722963866336$
2.12242208098718
Cl $9.49592589607334 \quad-4.47000974990315 \quad 1.66025048845880$
S $9.92590601771856 \quad-1.02211849352012 \quad 0.08811908660133$
$\begin{array}{llll}\text { C } & 10.96505534617346 & -1.22766385112465 & 1.54426286329716\end{array}$
H $\quad 11.75646910343797 \quad-0.48279888336672$
1.47302323862390
$\begin{array}{llll}\text { H } & 11.41670706780199 & -2.21440973296813 & 1.58770925638111\end{array}$
H $\quad 10.39795631105028 \quad-1.03856366920099 \quad 2.45275981682159$
C $8.60967190779168 \quad-5.33045705974518 \quad-1.25620992406804$
H $\quad 8.12038935669079 \quad-5.41275332191349 \quad-2.22105141891483$
H $8.26537888093255-6.13131852220589-0.60692266355129$
H $9.68643561067983 \quad-5.40426828658554 \quad-1.38814244983600$

\section{II-complex}

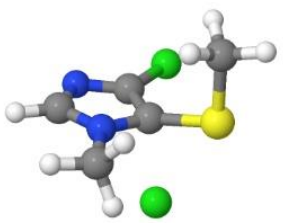

$$
\begin{array}{llll}
\mathrm{C} & 8.620947 & -2.438258 & 0.270577 \\
\mathrm{C} & 8.575729 & -3.848401 & 0.503732 \\
\mathrm{~N} & 7.636989 & -4.429471 & -0.227024 \\
\mathrm{C} & 7.101830 & -3.443010 & -0.943093 \\
\mathrm{~N} & 7.676268 & -2.255121 & -0.706311 \\
\mathrm{C} & 7.273470 & -0.979324 & -1.269765 \\
\mathrm{H} & 6.500271 & -1.164679 & -2.007467 \\
\mathrm{H} & 8.122731 & -0.497790 & -1.744300 \\
\mathrm{H} & 6.885283 & -0.344853 & -0.477314 \\
\mathrm{H} & 6.293288 & -3.560555 & -1.646011 \\
\mathrm{Cl} & 7.286165 & -1.941732 & 2.308765 \\
\mathrm{Cl} & 9.590372 & -4.692758 & 1.550282 \\
\mathrm{~S} & 9.878100 & -1.292865 & 0.648123 \\
\mathrm{C} & 10.974123 & -1.597999 & -0.762401 \\
\mathrm{H} & 11.817059 & -0.925501 & -0.622086 \\
\mathrm{H} & 10.480511 & -1.363303 & -1.700775 \\
\mathrm{H} & 11.327066 & -2.624861 & -0.760715
\end{array}
$$

\section{TSrot}

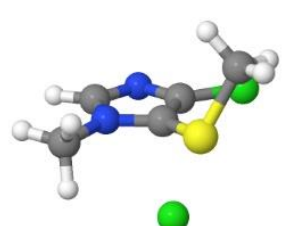




$\begin{array}{lllllllll}\text { C } & 8.64119695104245 & -2.33034379146178 & -0.02379452817167 & \text { H } & 6.05271475583168 & -3.53826668617443 & -1.49599679371198 \\ \text { C } & 8.68568920842740 & -3.74348698429479 & 0.21800914039951 & \text { Cl } 7.34021231712966 & -2.17920161503237 & 2.31438600977690 \\ \text { N } & 7.66370038976725 & -4.35911229555686 & -0.35632396195043 & \text { Cl } & 9.87144149242542 & -4.55422282946065 & 1.09558958825258 \\ \text { C } & 6.96559382142943 & -3.39287277529727 & -0.94193484370696 & \text { S } & 9.79932645741492 & -1.08092370058777 & 0.25040810127856 \\ \text { N } 7.51837484084193 & -2.17817299899157 & -0.78373715081283 & \text { C } & 11.27161446614774 & -1.77685304405547 & -0.54389617744880 \\ \text { C } & 6.96302410446800 & -0.92041426782041 & -1.25018827596640 & \text { H } & 12.01798631528324 & -0.99047797778354 & -0.46396486128623 \\ \text { H } & 6.14046988891837 & -1.14216560005207 & -1.92125559734038 & \text { H } & 11.07513314652479 & -1.98837611896639 & -1.58944769744808 \\ \text { H } & 7.72898707948662 & -0.35880738547556 & -1.77582542939991 & \text { H } & 11.62289001390231 & -2.66001357123025 & -0.02292734130241 \\ \text { H } & 6.60240175095877 & -0.34771435775883 & -0.39945518116145 & & & & & \end{array}$

\subsection{Computational Determination of Redox Potentials}

All computations were carried out using the ORCA 4.1.1 software package. ${ }^{52}$ Redox potentials were calculated in analogy to an established method by Nicewicz and coworkers. ${ }^{59}$ The geometries of all relevant structures were optimized using Handy's range-separated CAMB3LYP functional ${ }^{53}$ and Grimme's D3 dispersion correction ${ }^{54}$ in the def2-TZVPP basis set, as developed by Ahlrichs and co-workers, ${ }^{55}$ with the CPCM continuum solvation model ${ }^{56}$ (presets for Acetonitrile). No internal coordinate or symmetry constraints were applied. Optimized geometries were verified to be local minima on the respective potential energy landscape by the absence of negative eigenvalues of the Hessian, as obtained from a harmonic frequency calculation at the same level.

Free enthalpies were calculated from the electronic energy at the stationary point, corrected by zero-point vibrational energy (ZPVE), thermal, enthalpy and entropy corrections, as obtained from the harmonic calculations (see Table S8). All geometries were visualized using Jmol. ${ }^{57}$

Redox potentials were calculated from the Free Enthalpies of the respective oxidized and reduced species; the values were referenced to SCE by subtraction of its absolute potential $E_{\text {ref }}$ $(4.422 \mathrm{~V}) .{ }^{60}$

$$
E=-\frac{G_{298}(\mathrm{red})-G_{298}(\mathrm{ox})}{F}-E_{\mathrm{ref}}
$$

Redox potentials were determined for the reduction of several heteroaryl halides employed in this study (1a, 1b, 1f, 1n, 1q, 1v):

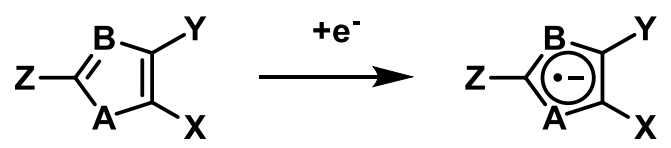

The computationally determined redox potentials are given in Table S7. All potentials were found to be lower than $-1.5 \mathrm{~V}$ (several even lower than $-2.0 \mathrm{~V}$ ). The photocatalyst employed in this study ([Ir(dFCF 3 ppy $\left.\left.)_{2}(\mathrm{dtbbpy})\right] \mathrm{PF}_{6}\right)$ has an excited state redox potential of $E_{1 / 2}\left(\mathrm{M}^{+} / \mathrm{M}^{*}\right)$ $=-0.89 \mathrm{~V}$ and a ground state potential of $E_{1 / 2}\left(\mathrm{M} / \mathrm{M}^{-}\right)=-1.37 \mathrm{~V} \cdot{ }^{15 \mathrm{c}}$ These potentials do not account for the reduction of any of the investigated heteroaryl halides. Therefore, a mechanism

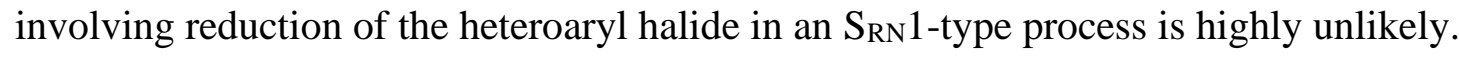


Table S7. Calculated redox potentials for the reduction of heteroaryl halides to the respective radical anions. All potentials given in $\mathrm{MeCN}$ referenced to SCE.

(E)

Table S8. Stationary point energies of the relevant heteroaryl halides and their respective radical anions. All energies are given in Hartree $\left(E_{\mathrm{h}}\right)$.

\begin{tabular}{lcccccc}
\hline & $\begin{array}{c}\text { Electronic } \\
\text { Energy / } E_{\mathrm{h}}\end{array}$ & ZPVE / $E_{\mathrm{h}}$ & $\begin{array}{c}\text { Thermal } \\
\text { Corr. / } E_{\mathrm{h}}\end{array}$ & $\begin{array}{c}\text { Enthalpy } \\
\text { Corr. / } E_{\mathrm{h}}\end{array}$ & $\begin{array}{c}\text { Entropy } \\
\text { Corr. / } E_{\mathrm{h}}\end{array}$ & $\begin{array}{c}\text { Free } \\
\text { Enthalpy / } E_{\mathrm{h}}\end{array}$ \\
\hline $\mathbf{1 a}$ & -1184.76907 & 0.0815579 & 0.007435 & 0.00094421 & 0.04126094 & -1184.72039 \\
$\mathbf{1 a}^{\cdot-}$ & -1184.85350 & 0.07910966 & 0.00821266 & 0.00094421 & 0.04397032 & -1184.80921 \\
$\mathbf{1 b}$ & -5412.87456 & 0.08032815 & 0.00790997 & 0.00094421 & 0.04370784 & -5412.82908 \\
$\mathbf{1 b ^ { - - }}$ & -5412.97155 & 0.07781854 & 0.00890319 & 0.00094421 & 0.04722739 & -5412.93111 \\
$\mathbf{1 f}$ & -7986.55031 & 0.06978022 & 0.00955829 & 0.00094421 & 0.04836697 & -7986.51840 \\
$\mathbf{1 f}{ }^{-}$ & -7986.65322 & 0.06778094 & 0.01020569 & 0.00094421 & 0.05068767 & -7986.62498 \\
$\mathbf{1 n}$ & -5700.36835 & 0.04765487 & 0.00675846 & 0.00094421 & 0.04147009 & -5700.35447 \\
$\mathbf{1 n}{ }^{--}$ & -5700.47057 & 0.04549511 & 0.0075811 & 0.00094421 & 0.04438093 & -5700.46093
\end{tabular}




\begin{tabular}{lllllll}
$\mathbf{1 q}$ & -5608.39268 & 0.13275716 & 0.01096373 & 0.00094421 & 0.05104813 & -5608.29906 \\
$\mathbf{1 q}^{\cdot-}$ & -5608.45917 & 0.12805435 & 0.01183082 & 0.00094421 & 0.05327807 & -5608.37162 \\
$\mathbf{1 v}$ & -3198.11305 & 0.06273085 & 0.00675659 & 0.00094421 & 0.03993085 & -3198.08255 \\
$\mathbf{1 \mathbf { v } ^ { - - }}$ & -3198.21198 & 0.06054226 & 0.00768237 & 0.00094421 & 0.04328522 & -3198.18609 \\
$\mathbf{1 s}$ & -1859.35418 & 0.1653496 & 0.01366719 & 0.00094421 & 0.05506403 & -1859.22928 \\
$\mathbf{1 s}^{--}$ & -1859.40531 & 0.16046137 & 0.01433247 & 0.00094421 & 0.05697369 & -1859.28654 \\
\hline
\end{tabular}

\section{1a}

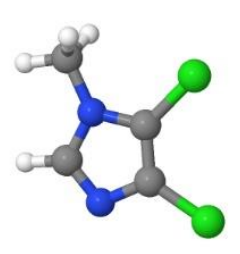

C $8.33649916501336 \quad-2.04137897780873$

C $8.45559649049157 \quad-3.39814937198258$

N $7.36981043147763 \quad-3.94770820647311$

C $6.59675075662592 \quad-2.93169123979394$

N $7.13450864211106-1.75095946906122$

C $6.55673615270977-0.43467925084010$

H 5.59999558858845

H 7.21052339854294

H 6.40596836708305

H 5.64066199323413

Cl 9.37223898476464

Cl 9.75913002935747
$-0.55581165945525-1.16479537139496$

$0.17526620629240 \quad-1.28551133977653$ $\begin{array}{ll}0.05099615987314 & 0.29305270067135\end{array}$

$-2.99416303477073-1.33607166563097$

$\begin{array}{ll}-0.84919762867099 & 0.73334805730313\end{array}$

$-4.33800352730889 \quad 0.65278393481408$

\section{$1 a^{\cdot-}$}

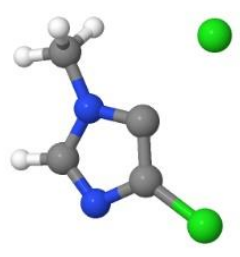

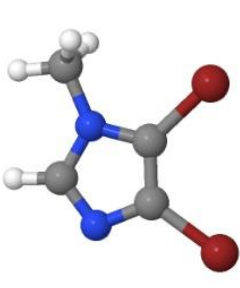

\section{$1 b$}
C 8.32242555160073
$-2.04596995288789$
0.10428589567720
C 8.43389770232943
$-3.40412663379467$
0.05305649836035
N $7.34495554620812-3.94684370121663$
$-0.54442977563716$
C 6.57699657483780
$-2.92608372317660$
$-0.85415786046238$
N 7.12065955709831
$-1.74925805553946$
$-0.48385805708419$
C 6.54100195513346
$-0.43365100115272$
$-0.67548512490114$
H 5.58398947292752
$-0.55689013006427$
$-1.17169310290474$
H 7.19309974670555
0.17643258686050
$-1.29493621383176$
H 6.39014919413173
0.05300878756910
0.28453030230672
H 5.62036220036160
$-2.98289042044475$
$-1.34588672393408$ 


\begin{abstract}
Br 9.46266105690800
Br 9.84822144175775

0.78772808643692

0.68985607597425
\end{abstract}

$1 b^{\cdot-}$

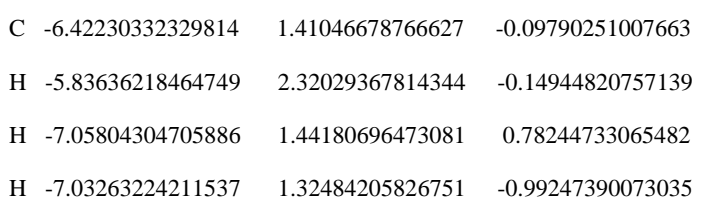

$1 \mathbf{f}^{-}$

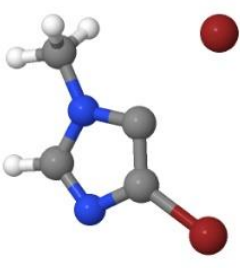

C 8.30508618822838

C 8.36464485602123

N 7.22441890448090

C 6.47048570165884

N 7.08110685593485

C 6.52708131832410

H 5.54956217936231

H 7.17656938885241

H 6.42299120166328

H 5.48329896234196

Br 10.04312893058512

Br 9.79046551254661

$-2.09307771981230$

$-3.45155312713292$

$-4.01354576933108$

$-2.98639967933073$

$-1.80920891667937$

$-0.49038919891975$

$-0.60022841116658$

0.07839594167398

0.04388890464328

$-3.04066993860708$

$-0.13607943592763$

$-4.51871264940981$

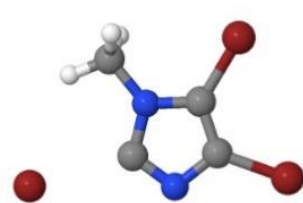

0.06938769328710

0.08260915247247

$-0.41755304135420$

$-0.74153197281473$

$-0.46899423672150$

$-0.69842107941253$

$-1.15944453811694$

$-1.35823036205108$

0.24260316256321

$-1.17122516644698$

0.46593160338510

0.69140878521008

$\begin{array}{llll}\mathrm{N} & -5.44738489935818 & 0.28121917318270 & -0.03\end{array}$

1f

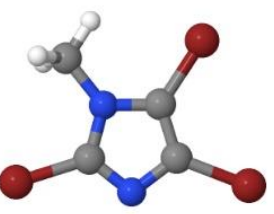

$\begin{array}{llll}\text { C } & -4.07551345929073 & 0.33363713149408 & 0.10161974426712\end{array}$

$\begin{array}{llll}\text { C } & -3.65703202166564 & -0.96012325124789 & 0.10665796006677\end{array}$

N $\quad-4.72233290136967 \quad-1.80503874740544 \quad-0.01883514071383$

C $\quad-5.76839779763963 \quad-1.02736290334612 \quad-0.10285301831160$

Br $-8.37025643836053 \quad-1.79515849661553 \quad-0.10948278800628$

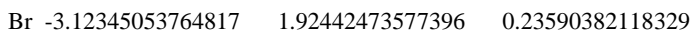

Br $-1.89729906563098 \quad-1.58115143682884 \quad 0.26111387549828$

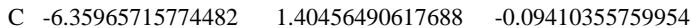

H $\quad-6.13174357149200 \quad 2.03107718432914 \quad-0.95343831763482$

H $\quad-6.28874833487331 \quad 1.99873077868708 \quad 0.81401540932098$

$\begin{array}{llll}\text { H } & -7.36251381492632 & 1.00351092579997 & -0.18931489194170\end{array}$

$\begin{array}{llll}\text { N } & -5.51604821103608 & 0.27775085934768 & -0.01033815670002\end{array}$

$\begin{array}{llll}\text { C } & -4.13904365810514 & 0.29637742790349 & 0.00939624579447\end{array}$

C $\quad-3.74792129908857-1.00523782201271 \quad 0.09993093211028$

$\begin{array}{llll}\mathrm{N} & -4.82961411492812 & -1.82621708378784 & 0.13670557658035\end{array}$

C $\quad-5.85824956622783 \quad-1.02665707628430 \quad 0.06918162479521$

Br -7.63329702531595 $-1.58675767213481 \quad 0.07970188710923$

$\begin{array}{llll}\mathrm{Br} & -3.12684281277914 & 1.846687773578966 & -0.07566302815039\end{array}$

$\begin{array}{llll}\mathrm{Br} & -2.00370251539931 & -1.66464585762920 & 0.16889220618442\end{array}$

$1 \mathbf{n}$

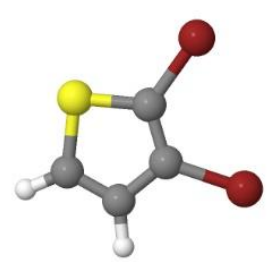

$\begin{array}{cccc}\text { C } & 8.57259933403345 & -1.82503090668583 & 0.64100544296026 \\ \text { C } & 8.98644831992450 & -3.02949403114552 & 0.16777009360574 \\ \text { C } & 7.94134813397322 & -3.77269230044251 & -0.44403996520685 \\ \text { C } & 6.76152512103568 & -3.10642017153878 & -0.41595931250292 \\ \text { S } & 6.90128684026500 & -1.58205117081208 & 0.34609246442558 \\ \text { Br } 9.58651630398127 & -0.52402497127499 & 1.51232243653620 \\ \text { Br } 10.74493638302629 & -3.67476674489731 & 0.29261777225542\end{array}$



H 8.08010458355125
$-0.80376611813903$
$1 n^{\cdot-}$

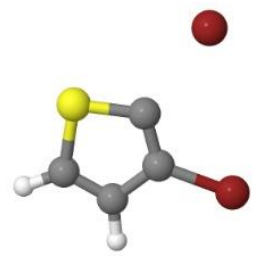
C $8.54203217501740 \quad-1.88718276601282$
0.60932584508214
C $8.93897895733808 \quad-3.07226598260992$
0.10309943062036
C $7.89622020439451 \quad-3.82326852482091$
$-0.51955346654016$
C $6.70867408361119-3.16904447826503$
$-0.46370390127410$
S $6.87944524170210-1.65584701156492$
Br 9.90366106984987 -0.07966977593071
0.34293133082198
Br $10.72106420794271 \quad-3.72428523216648$
1.98459983269315
H $8.03912388957873-4.79101794687658$
H $5.75549017056542-3.49170828175261$
0.18858613750968
$-0.97628100693569$
$-0.84934420197735$

\section{$1 q$}

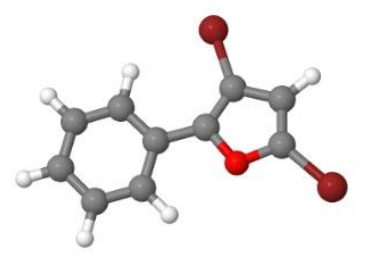
C 8.50271902039726
$-1.95100483070943$
0.55849858637181
C $9.09241265646565-3.09374492839265$
0.16344961004293
C 8.04916292529112
$-3.84710534404502$
$-0.45133447477271$
C 6.89973437982883
$-3.11851572583589$
$-0.39196053298497$
O $7.19637474836322-1.93920230545725$
0.24050989023282
C $5.51160405025661 \quad-3.27737935888678$
$-0.80615106201306$
Br 9.15991031253613
$-0.45406342530530$
1.43244039664920
Br $8.33009736794333 \quad-5.55121900916978$
$-1.17192392250552$
C $5.08417691903652-4.37195251632656$
$-1.55757549488092$
C 4.57438983474530
$-2.30639086910719$
$-0.44619862893172$
C 3.75798033969555
$-4.48945042077611$

\begin{abstract}
C $3.25128016612990 \quad-2.43011565439419$
$-0.82614189716481$

C $2.83501159193021 \quad-3.52223018949411 \quad-1.57141375807475$

H $1.79986244352923 \quad-3.61750781986295 \quad-1.86831710282845$

H $3.44708959629471 \quad-5.34499408905301 \quad-2.51731279832572$

$\begin{array}{llll}\text { H } & 4.88420049316270 & -1.45148767880016 & 0.13518571425481\end{array}$

H $2.54125246510578 \quad-1.66792445546868 \quad-0.53628174669760$

H $5.78288765496104 \quad-5.13557160099176 \quad-1.85639816355956$

$\begin{array}{llll}\text { H } & 10.12111303432693 & -3.38020977792317 & 0.28496283825173\end{array}$
\end{abstract}

$1 q^{\cdot-}$

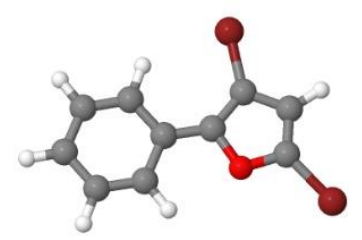
C $8.52566682762870-1.94623124821434$
0.57073847961049
$\begin{array}{lll}\text { C } 9.10347937444377 & -3.09687855687429\end{array}$
0.16415577789595
C $8.07718544012067 \quad-3.85060779066447$
$-0.44718272293434$
C $6.87732096268558-3.12151222441809$
$-0.39434443565014$
O $7.19616379418269-1.91816788366122$
0.25773637447297
C $5.54325572464580 \quad-3.27528334767873$
$-0.79564840646274$
Br 9.19743923176905
$-0.44949797354994$
1.45117837310449
Br $8.37151534011107 \quad-5.55664626011901$
$-1.18789110722583$
C $5.04873681782181-4.43737003592933$
$-1.47267030328887$
C $4.57829549998653-2.24326188146493$
$-0.53982741145242$
C $3.73295029705816 \quad-4.54149282809964 \quad-1.84655620530684$
C $3.26742769457023 \quad-2.37606220701157 \quad-0.92621753647337$
C $2.80500574849040 \quad-3.52088157699655 \quad-1.58724602341559$
H $1.77124919932344 \quad-3.61497442627613 \quad-1.88768803790501$
H $3.40848977100472 \quad-5.44111417592752 \quad-2.35667918652674$
H $\quad 4.89304408036443 \quad-1.34332855531127 \quad-0.03210781030818$
H $2.57709440178885 \quad-1.56869974763025 \quad-0.71013995740585$
H $\quad 5.72018193816983 \quad-5.25125194448313 \quad-1.69668833819668$
H $10.13494785583426 \quad-3.37904733568960 \quad 0.283288477468873$ 


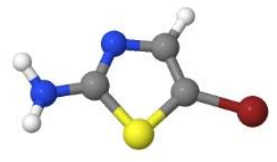

$\begin{array}{llll}\text { C } & 8.61316675364899 & -1.78657156272848 & 0.67133348590933\end{array}$

$\begin{array}{llll}\text { C } & 8.97502315830461 & -2.98618146300150 & 0.18216402976337\end{array}$

N $7.96319715025735 \quad-3.72364415343425 \quad-0.38408868422031$

C $6.82967641685383 \quad-3.08991440613097 \quad-0.32566360703595$

S $6.91597942298623 \quad-1.52398887857232 \quad 0.43399508246820$

Br $9.69662757438572 \quad-0.50915817137982 \quad 1.50056524862758$

N $5.65047773561835 \quad-3.59467389665120 \quad-0.75912409111345$

H $9.97851604658418 \quad-3.38204886011685 \quad 0.20779455047850$

H $5.71168071861985 \quad-4.38905681659548 \quad-1.37603643805170$

H $4.89343502274089-2.96033179138913 \quad-0.94876957682557$

\section{$1 \mathbf{v}^{\bullet-}$}

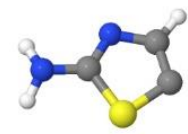

$\begin{array}{cccc}\text { C } & 8.57905533931064 & -1.84903127098774 & 0.64512166470210 \\ \text { C } & 8.93347764701475 & -3.03646641627008 & 0.12817621718351 \\ \text { N } & 7.92014018868847 & -3.74159942586472 & -0.51807372026345 \\ \text { C } & 6.80479664572906 & -3.08405480569984 & -0.48855727735156 \\ \text { S } & 6.91344639902386 & -1.54756904193718 & 0.33558027504043 \\ \text { Br } & 10.10343775416726 & -0.08229846875350 & 2.03289698081914 \\ \text { N } & 5.64929890961333 & -3.49807431726577 & -1.09568153782280 \\ \text { H } & 9.91822237269115 & -3.47669044036060 & 0.17846324120256 \\ \text { H } & 5.61905309369446 & -4.48525990554263 & -1.30102159679636 \\ \text { H } & 4.78685165006702 & -3.14452590731795 & -0.71473424671358\end{array}$

\section{1s}

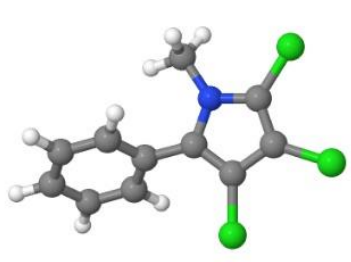

Jmol

\begin{tabular}{|c|c|c|c|}
\hline$C$ & 8.74762647128489 & -1.85055273539726 & 0.28389985949086 \\
\hline C & 9.00906880717536 & -3.18671845051852 & 0.42001095287845 \\
\hline E & 7.83542455951090 & -3.86509248272710 & 0.02725080151373 \\
\hline $\mathrm{C}$ & 6.89433750381683 & -2.92839612736173 & -0.32988447523093 \\
\hline I & 7.47344251692931 & -1.69067498463526 & -0.17057660556633 \\
\hline c & 6.87223232908065 & -0.41035251534985 & -0.50254357838726 \\
\hline I & 6.02520628847376 & -0.57988682955445 & -1.15643545589013 \\
\hline H & 7.59994679871216 & 0.20666824167552 & -1.02058191045132 \\
\hline H & 6.53758158800210 & 0.10448726089413 & 0.39561900384221 \\
\hline$=$ & 5.50811518167607 & -3.12655475407536 & -0.76879003589142 \\
\hline 1 & 9.77844183947438 & -0.53217613552478 & 0.59159442616681 \\
\hline $\mathrm{Cl}$ & 10.46677485214331 & -3.88767869450812 & 0.96979871718351 \\
\hline $\mathrm{Cl}$ & 7.61958239412176 & -5.56554234828499 & 0.06538555734109 \\
\hline & 5.23042095217633 & -3.96003207954750 & -1.84818129384299 \\
\hline C & 4.45245782833176 & -2.51181883216604 & -0.10023587159630 \\
\hline C & 3.92343386406931 & -4.17323396674449 & -2.25150064349108 \\
\hline & 3.14684298100950 & -2.72028300565591 & -0.50909221076239 \\
\hline $\mathrm{C}$ & 2.87891217349786 & -3.55224566532533 & -1.58501308515065 \\
\hline $\mathrm{H}$ & 1.85849663910548 & -3.71640715089938 & -1.90270671653751 \\
\hline $\mathrm{H}$ & 3.72157108390033 & -4.82167352128331 & -3.09283828572773 \\
\hline $\mathrm{H}$ & 4.65381612672057 & -1.87563327398337 & 0.75059919061485 \\
\hline $\mathrm{H}$ & 2.33660279890651 & -2.23682532739497 & 0.01891894631355 \\
\hline & 6.04385442188088 & -4.43702662163192 & -2.37621728681901 \\
\hline
\end{tabular}

$1 s^{\cdot-}$

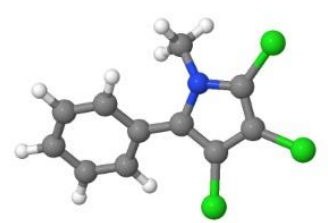

C $8.72246902034962 \quad-1.84419483723690 \quad 0.41228515596958$

$\begin{array}{llll}\text { C } & 9.08498962959146 & -3.14329140382383 & 0.23450994733049\end{array}$ 


$\begin{array}{llll}\text { C } & 7.96391872986134 & -3.83666284680902 & -0.26991731561278 \\ \text { C } & 6.88670853745745 & -2.93908146647612 & -0.37676924140888 \\ \text { N } & 7.39106565003298 & -1.69425388481241 & 0.05927139041745 \\ \text { C } & 6.85989418172470 & -0.42114660235465 & -0.39246169040015 \\ \text { H } & 6.27015755913055 & -0.58076226531275 & -1.29154001329406 \\ \text { H } & 7.68046326831819 & 0.25164238828991 & -0.62583654490676 \\ \text { H } & 6.22713442048074 & 0.05049943531281 & 0.35781255730723 \\ \text { C } & 5.54260097357766 & -3.13007548658966 & -0.77910264459793 \\ \text { Cl } & 9.63205308829434 & -0.56637359223571 & 1.09740699580737 \\ \text { Cl } & 10.59527224685586 & -3.83426950518421 & 0.66349870805307 \\ \text { Cl } 7.90130041220250 & -5.55385981095188 & -0.42318031468178\end{array}$

$\begin{array}{llll}\text { C } & 5.15995130379022 & -4.16917997368635 & -1.69465181856253 \\ \text { C } & 4.46352661803939 & -2.32934831417653 & -0.26640850723907 \\ \text { C } & 3.85487828231556 & -4.35731949832117 & -2.06602975125568 \\ \text { C } & 3.16444899691610 & -2.53976872266775 & -0.64982796912451 \\ \text { C } & 2.81588061497693 & -3.54946558547707 & -1.56450479213430 \\ \text { H } & 1.78939841238847 & -3.70502290829565 & -1.86431351159614 \\ \text { H } & 3.62376479864005 & -5.14597704382420 & -2.77328305498615 \\ \text { H } & 4.67262416468136 & -1.56207005770004 & 0.46551508692391 \\ \text { H } & 2.38829271693341 & -1.91488372567310 & -0.22228753500523 \\ \text { H } & 5.92339637344116 & -4.80278429199369 & -2.12170513700315\end{array}$




\subsection{Mechanistic Considerations Regarding the Methylthiolation}

In a previous study from our group, we could show that disulfides quench the photocatalyst $\left[\operatorname{Ir}\left(\mathrm{dFCF}_{3} \mathrm{ppy}\right)_{2}(\mathrm{dtbbpy}) \mathrm{PF}_{6}\right.$ via an energy transfer mechanism (no reductive quenching was observed) to give two thiyl radicals. ${ }^{14 \mathrm{~d}}$ Through this activation pathway, disulfides could be used instead of thiols in radical thiol-ene reactions. Termination events were found to proceed via hydrogen atom abstraction either from the solvent or from the $\alpha-\mathrm{C}-\mathrm{H}$ of the disulfide to yield polysulfides.

While initiation of the heterocycle thiolation using dimethyl disulfide could be readily explained by energy-transfer-mediated $\mathrm{S}-\mathrm{S}$ homolysis, a hypothetical propagation of that radical chain reaction with the disulfide seems unlikely. Instead, a formal disproportionation of the disulfide to the respective thiol and polysulfides can be postulated, in a similar mechanistic scenario as in the thiol-ene reaction discussed above. The methane thiol formed via this process would then enable chain propagation.

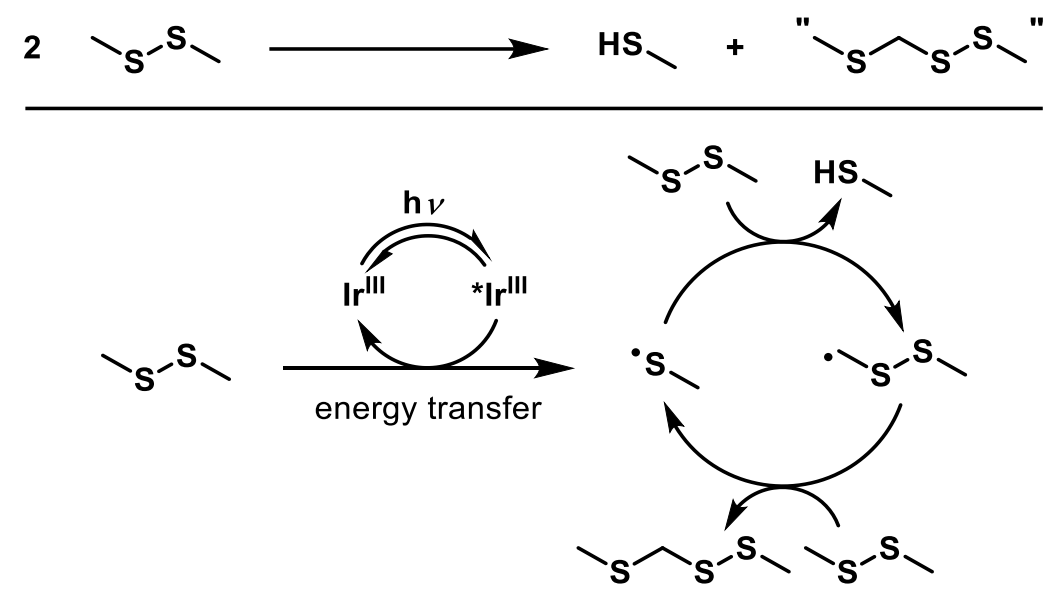

To get some evidence for this kind of disproportionation event, we conducted the following NMR experiment analogous to our standard reaction conditions:

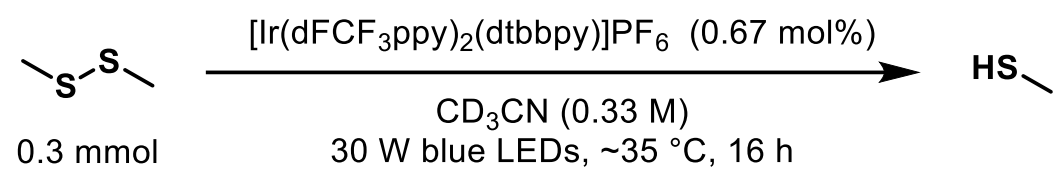

$\left[\operatorname{Ir}\left(\mathrm{dFCF}_{3} \mathrm{ppy}\right)_{2}(\mathrm{dtbbpy})\right] \mathrm{PF}_{6}(2.2 \mathrm{mg}, 0.002 \mathrm{mmol}, 0.67 \mathrm{~mol} \%)$ was weighed into an oven-dried Schlenk tube containing a magnetic stirring bar. The tube was evacuated and backfilled with argon three times. $\mathrm{CD}_{3} \mathrm{CN}(1 \mathrm{~mL}, 0.33 \mathrm{M})$ was added, followed by dimethyl disulfide $(26.7 \mu \mathrm{L}$, $0.30 \mathrm{mmol}, 1.0$ equiv). The solution was degassed via three freeze-pump-thaw cycles and transferred into a Young Teflon NMR tube under argon. The NMR tube was sealed and the reaction was stirred under irradiation with $30 \mathrm{~W}$ blue LEDs at room temperature for $16 \mathrm{~h}$. After irradiation, the ${ }^{1} \mathrm{H}$ NMR spectrum was recorded: 


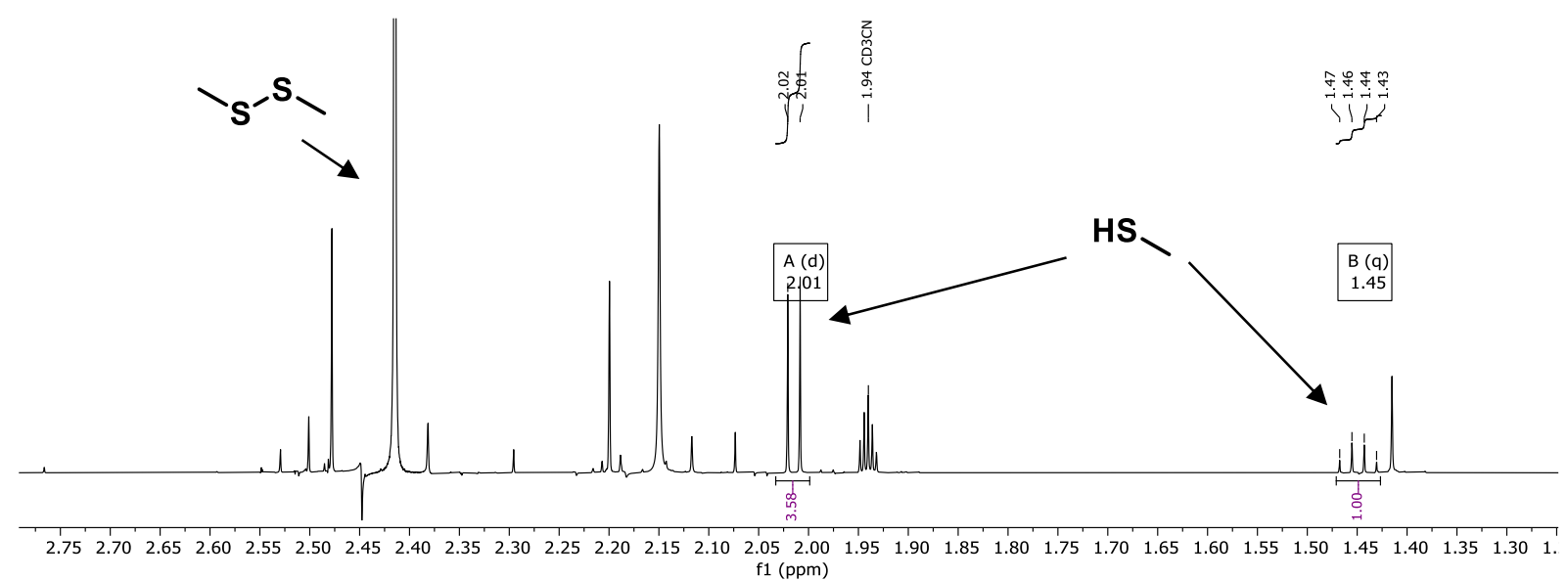

Formation of significant amounts of methane thiol along with possible polysulfide side products could be observed, supporting the mechanistic hypothesis suggested above. 


\section{Analytical Data}

\subsection{X-Ray Data}

\section{X-ray crystal structure analysis of $\mathbf{3 g}$}

A colorless needle-like specimen of $\mathrm{C}_{10} \mathrm{H}_{6} \mathrm{Br}_{2} \mathrm{Cl}_{2} \mathrm{~N}_{2} \mathrm{~S}$, approximate dimensions $0.020 \mathrm{~mm} \mathrm{x}$ $0.060 \mathrm{~mm} \times 0.200 \mathrm{~mm}$, was used for the X-ray crystallographic analysis. The X-ray intensity data were measured. A total of 1724 frames were collected. The total exposure time was 17.67 hours. The frames were integrated with the Bruker SAINT software package using a wideframe algorithm. The integration of the data using a monoclinic unit cell yielded a total of 19238 reflections to a maximum $\theta$ angle of $66.72^{\circ}$ ( $0.84 \AA$ resolution), of which 2265 were independent (average redundancy 8.494, completeness $=99.9 \%, \mathrm{R}_{\text {int }}=6.24 \%, \mathrm{R}_{\text {sig }}=3.34 \%$ ) and $1991(87.90 \%)$ were greater than $2 \sigma\left(\mathrm{F}^{2}\right)$. The final cell constants of $\underline{a}=11.4708(3) \AA$ 7.5086(2) $\AA, \underline{c}=15.4491(4) \AA, \beta=106.0300(10)^{\circ}$, volume $=1278.89(6) \AA^{3}$, are based upon the refinement of the XYZ-centroids of 6351 reflections above $20 \sigma(\mathrm{I})$ with $8.568^{\circ}<2 \theta<$ $133.0^{\circ}$. Data were corrected for absorption effects using the multi-scan method (SADABS). The ratio of minimum to maximum apparent transmission was 0.565 . The calculated minimum and maximum transmission coefficients (based on crystal size) are 0.1770 and 0.7780 . The structure was solved and refined using the Bruker SHELXTL Software Package, using the space group $P 2_{1} / n$, with $\mathrm{Z}=4$ for the formula unit, $\mathrm{C}_{10} \mathrm{H}_{6} \mathrm{Br}_{2} \mathrm{Cl}_{2} \mathrm{~N}_{2} \mathrm{~S}$. The final anisotropic full-matrix least-squares refinement on $\mathrm{F}^{2}$ with 155 variables converged at $\mathrm{R} 1=3.02 \%$, for the observed data and $\mathrm{wR} 2=7.50 \%$ for all data. The goodness-of-fit was 1.031 . The largest peak in the final difference electron density synthesis was $0.667 \mathrm{e}^{-} / \AA^{3}$ and the largest hole was $-0.602 \mathrm{e}^{-} / \AA^{3}$ with an RMS deviation of $0.111 \mathrm{e}^{-} / \AA^{3}$. On the basis of the final model, the calculated density was $2.166 \mathrm{~g} / \mathrm{cm}^{3}$ and $\mathrm{F}(000), 800 \mathrm{e}^{-}$. CCDC No.: 1981863.

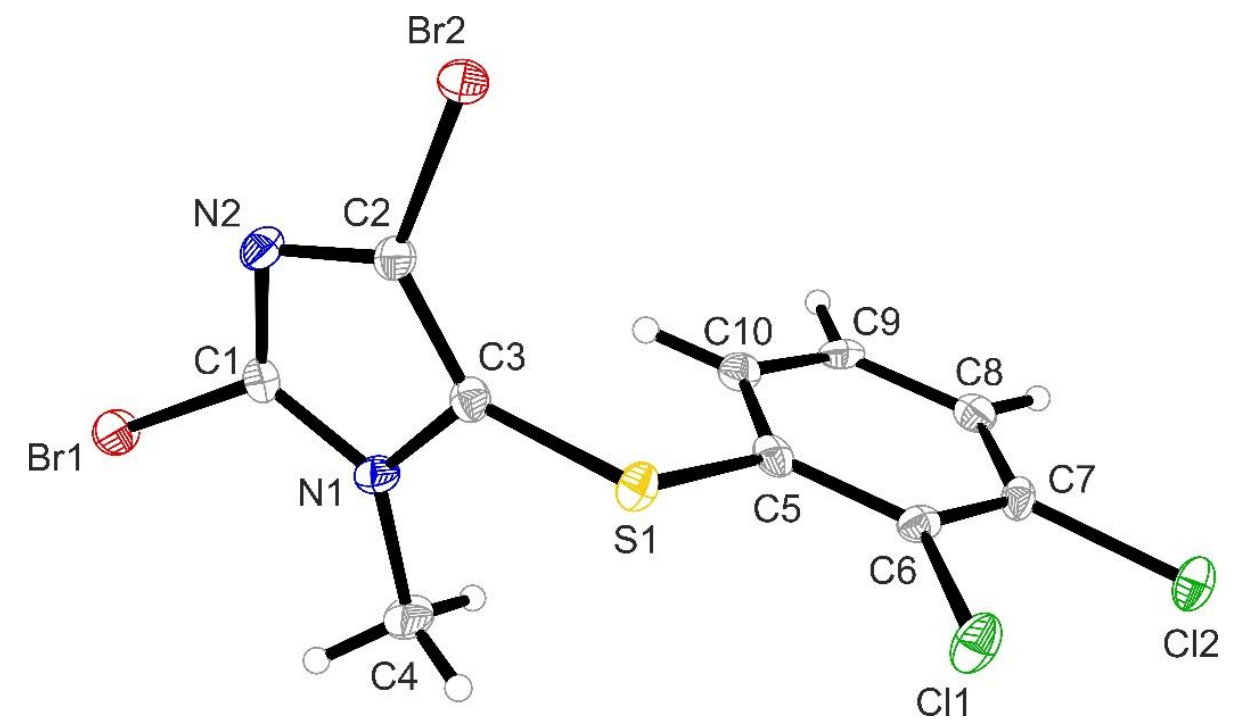

Figure S12. Crystal structure of compound 3g. Thermal ellipsoids are shown at 50\% probability. 


\section{X-ray crystal structure analysis of 3ae}

A colorless needle-like specimen of $\mathrm{C}_{10} \mathrm{H}_{7} \mathrm{Cl}_{3} \mathrm{~N}_{2} \mathrm{~S}$, approximate dimensions $0.039 \mathrm{~mm} \times 0.045$ $\mathrm{mm} \times 0.299 \mathrm{~mm}$, was used for the X-ray crystallographic analysis. The X-ray intensity data were measured. A total of 390 frames were collected. The total exposure time was 2.17 hours. The frames were integrated with the Bruker SAINT software package using a narrow-frame algorithm. The integration of the data using a monoclinic unit cell yielded a total of 17515 reflections to a maximum $\theta$ angle of $27.14^{\circ}$ ( $0.78 \AA$ resolution), of which 2597 were independent (average redundancy 6.744, completeness $=99.3 \%, \mathrm{R}_{\text {int }}=4.19 \%, \mathrm{R}_{\text {sig }}=2.39 \%$ ) and $2353(90.60 \%)$ were greater than $2 \sigma\left(\mathrm{F}^{2}\right)$. The final cell constants of $\underline{a}=13.6786(4) \AA$ 7.3704(2) $\AA, \underline{c}=13.2706(3) \AA, \beta=118.2240(10)^{\circ}$, volume $=1178.83(5) \AA^{3}$, are based upon the refinement of the XYZ-centroids of 7228 reflections above $20 \sigma(\mathrm{I})$ with $6.479^{\circ}<2 \theta<$ $54.26^{\circ}$. Data were corrected for absorption effects using the multi-scan method (SADABS). The ratio of minimum to maximum apparent transmission was 0.930 . The calculated minimum and maximum transmission coefficients (based on crystal size) are 0.7700 and 0.9650 . The structure was solved and refined using the Bruker SHELXTL Software Package, using the space group $P 2_{1} / c$, with $\mathrm{Z}=4$ for the formula unit, $\mathrm{C}_{10} \mathrm{H}_{7} \mathrm{Cl}_{3} \mathrm{~N}_{2} \mathrm{~S}$. The final anisotropic full-matrix least-squares refinement on $\mathrm{F}^{2}$ with 146 variables converged at $\mathrm{R} 1=2.40 \%$, for the observed data and $\mathrm{wR} 2=5.89 \%$ for all data. The goodness-of-fit was 1.060 . The largest peak in the final difference electron density synthesis was $0.333 \mathrm{e}^{-} / \AA^{3}$ and the largest hole was $-0.228 \mathrm{e}^{-} / \AA^{3}$ with an RMS deviation of $0.052 \mathrm{e}^{-} / \AA^{3}$. On the basis of the final model, the calculated density was $1.654 \mathrm{~g} / \mathrm{cm}^{3}$ and $\mathrm{F}(000), 592 \mathrm{e}^{-}$. CCDC No.: 1981864.

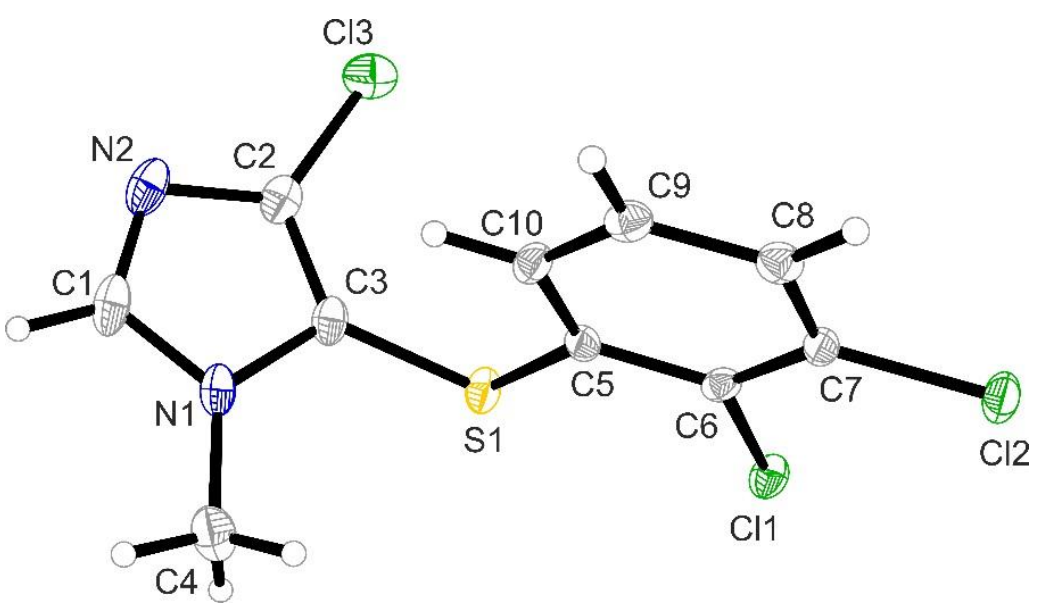

Figure S13. Crystal structure of compound 3ae. Thermal ellipsoids are shown at 50\% probability. 
5.2. NMR Data 
1-Benzyl-2-bromo-4,5-dichloro-1 $H$-imidazole (1h)

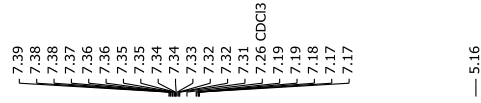

(1)

$\stackrel{1 \mathrm{~h}}{1 \mathrm{H} N M R, \mathrm{CDC}_{3}, 400 \mathrm{MHz}}$

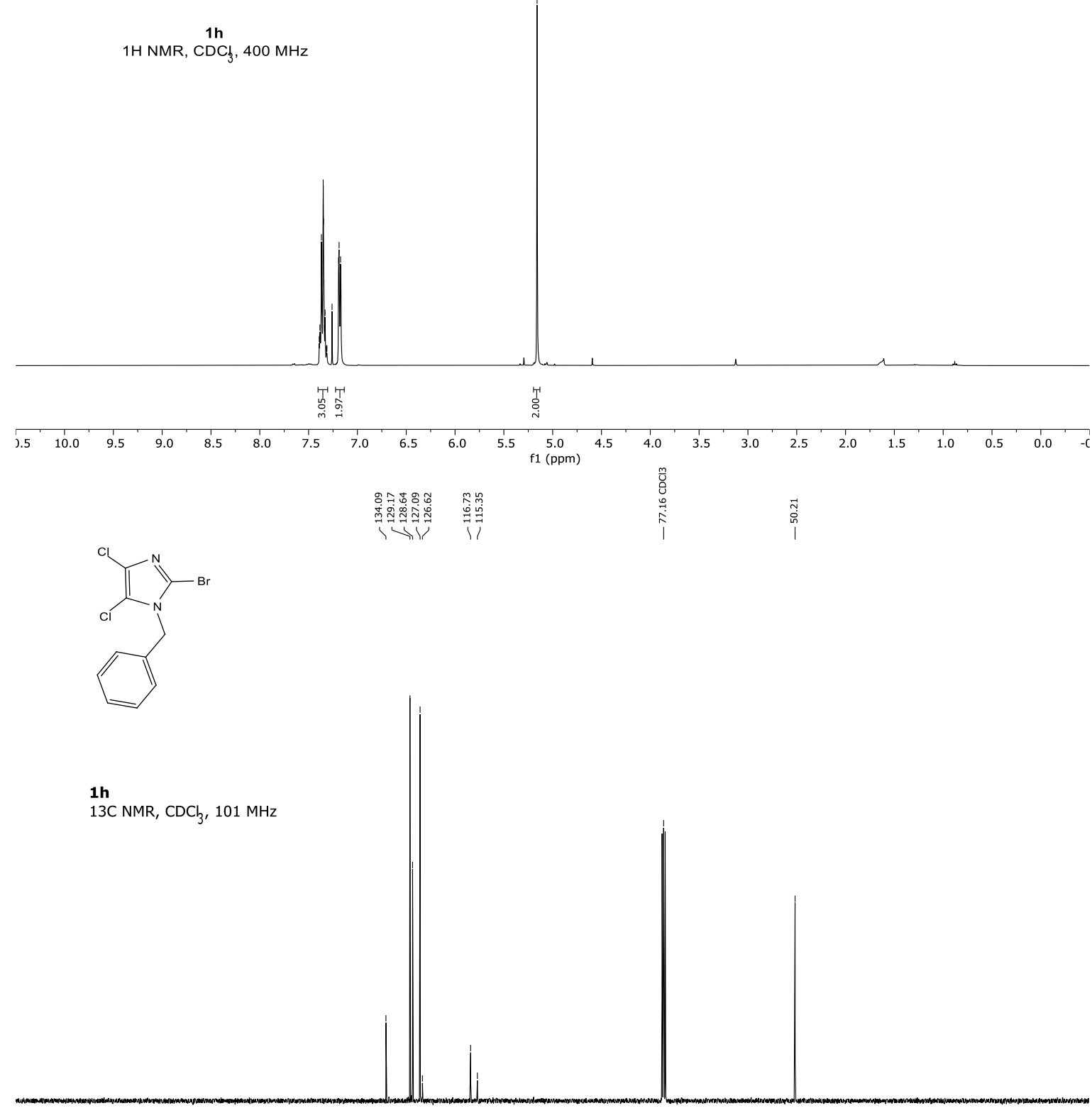

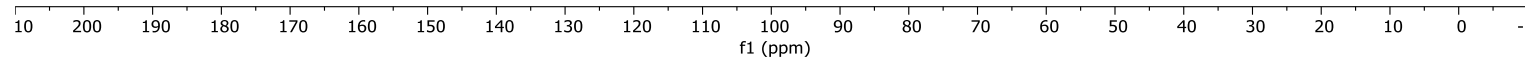


Methyl

(R)-2-((tert-butoxycarbonyl)amino)-3-(5-chloro-1-methyl-1H-imidazol-4-

yl)propanoate (1i)

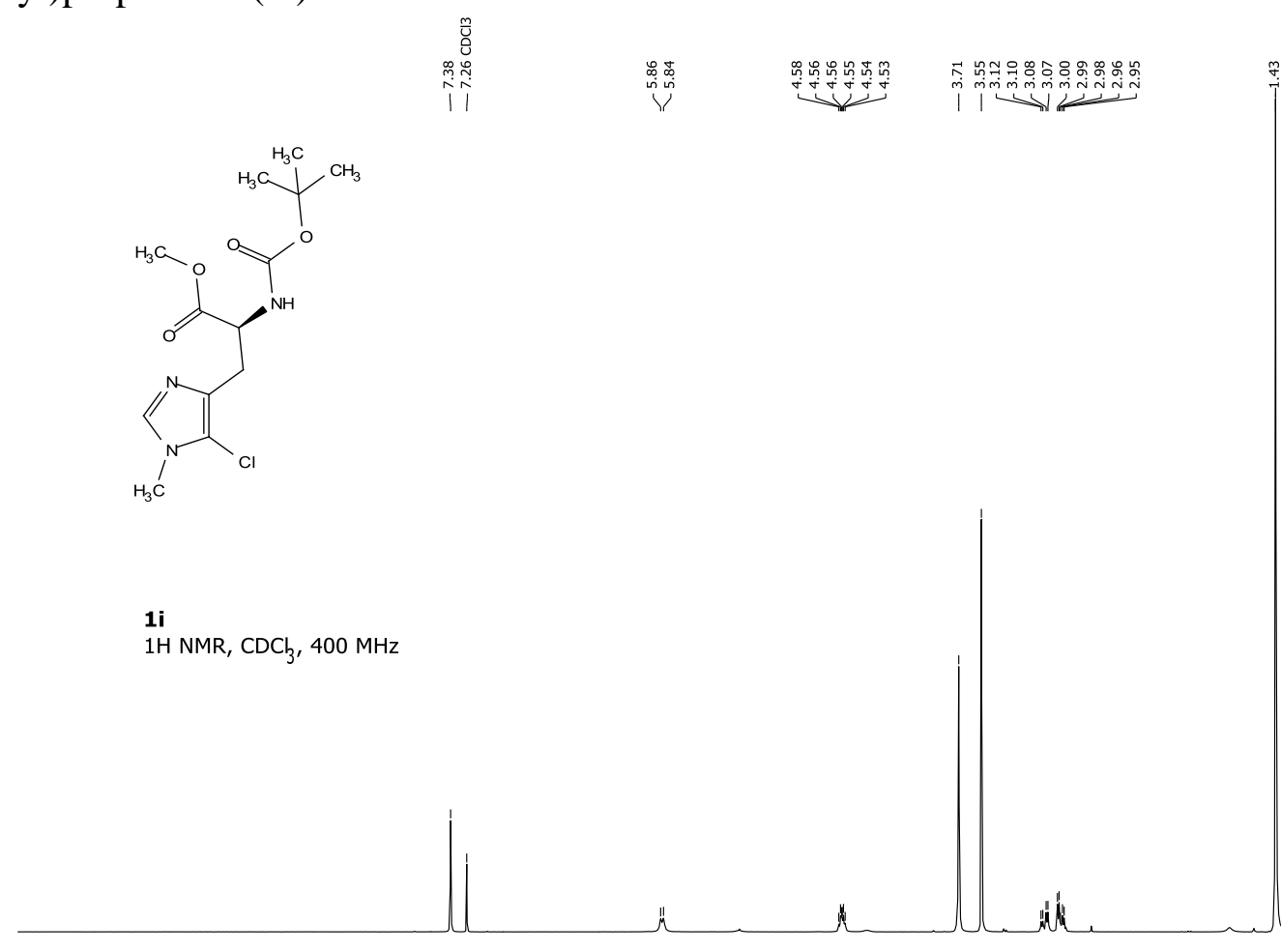

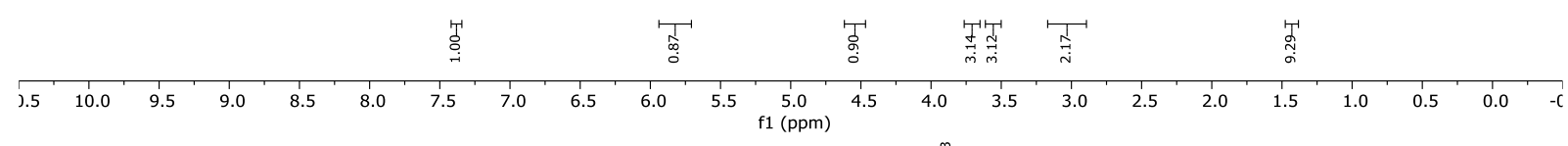
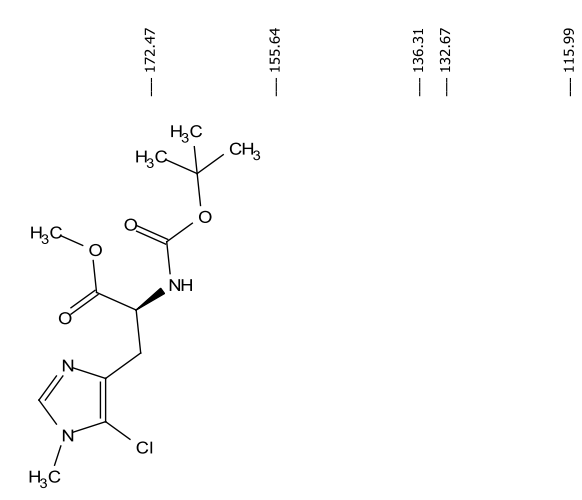

$13 \mathrm{C} \mathrm{NMR}, \mathrm{CDCl}_{3}, 101 \mathrm{MHz}$

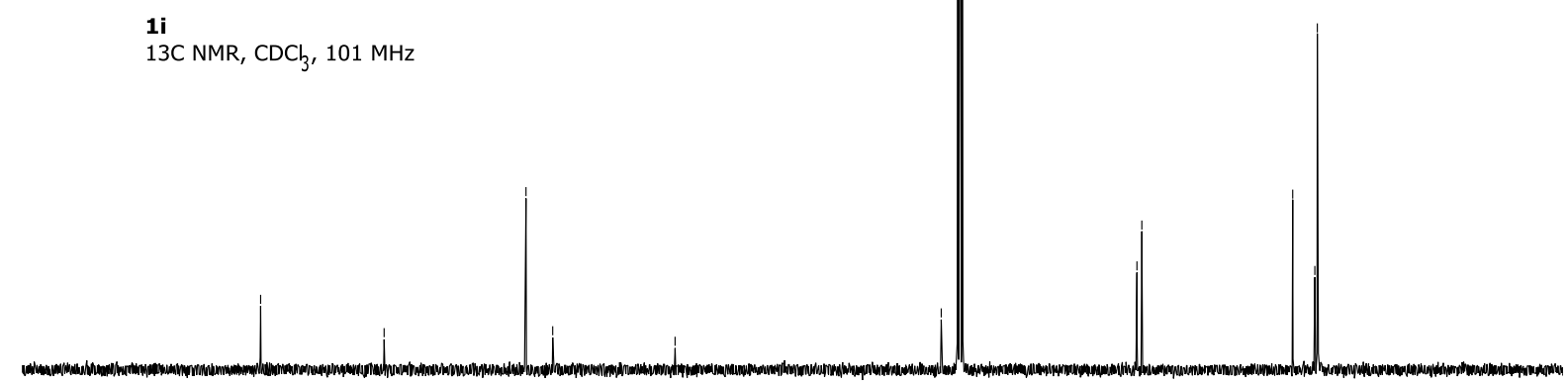

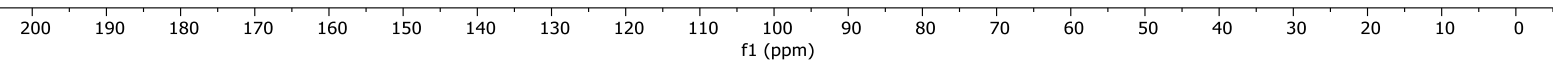


Methyl

(S)-2-((tert-butoxycarbonyl)amino)-3-(2,5-dichloro-1-methyl-1H-imidazol-4yl)propanoate $(\mathbf{1} \mathbf{j})$
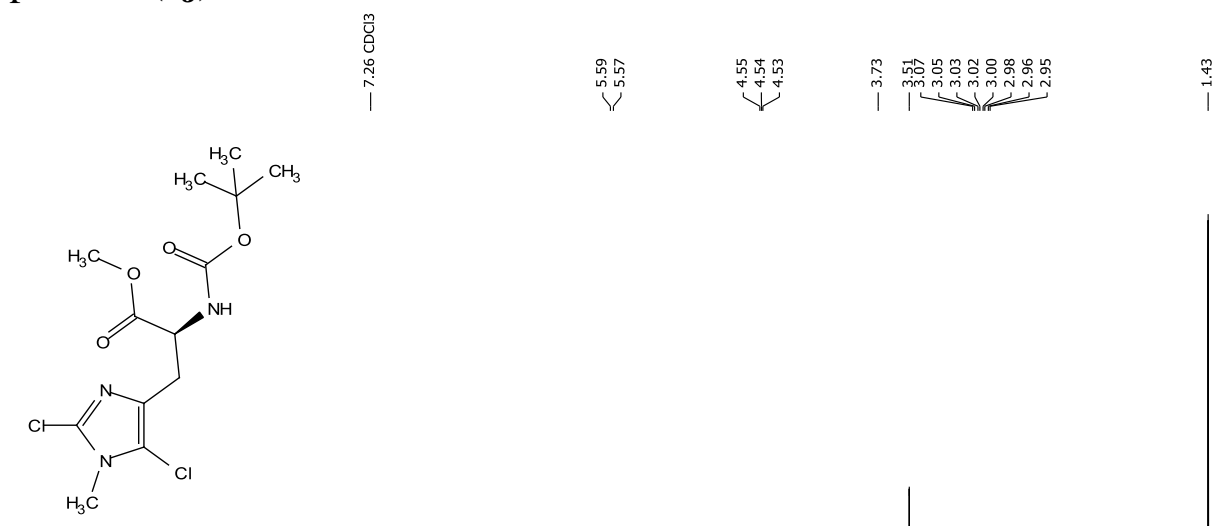

1j

$1 \mathrm{H} N M R, \mathrm{CDCl}_{3}, 400 \mathrm{MHz}$

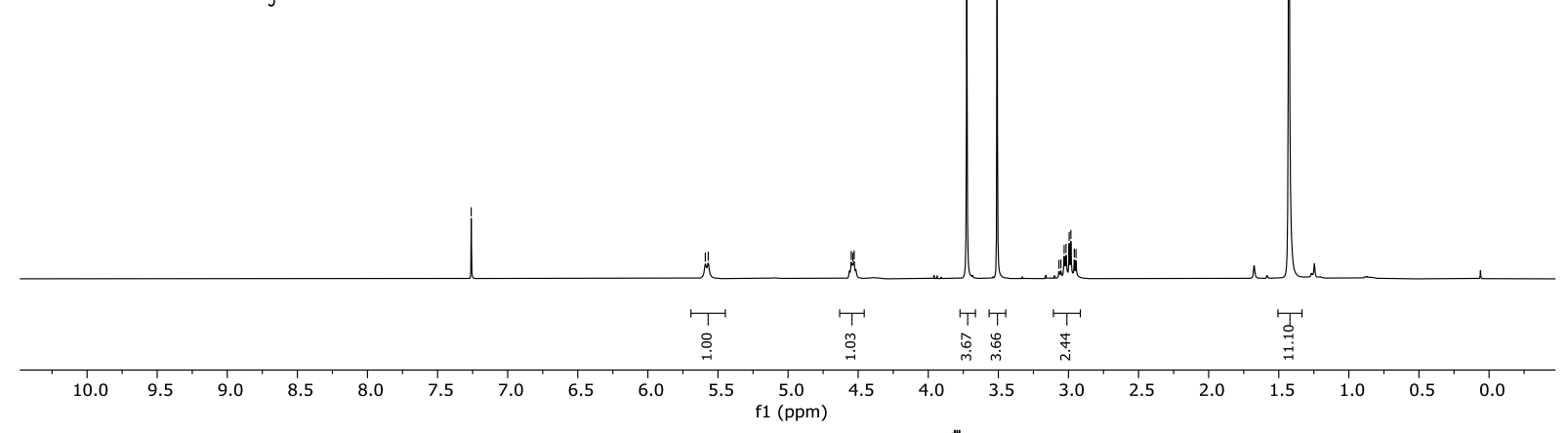<smiles>COC(=O)[C@H](Cc1nc(Cl)n(C)c1Cl)NC(=O)OC(C)(C)C</smiles>

$\mathbf{1 j}$
$13 \mathrm{C} \mathrm{NMR}, \mathrm{CDCl}_{3}, 101 \mathrm{MHz}$

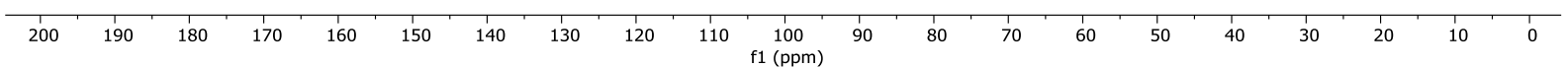


4,5-Dichloro-1-(2-chlorobenzyl)-1H-imidazole (1k)

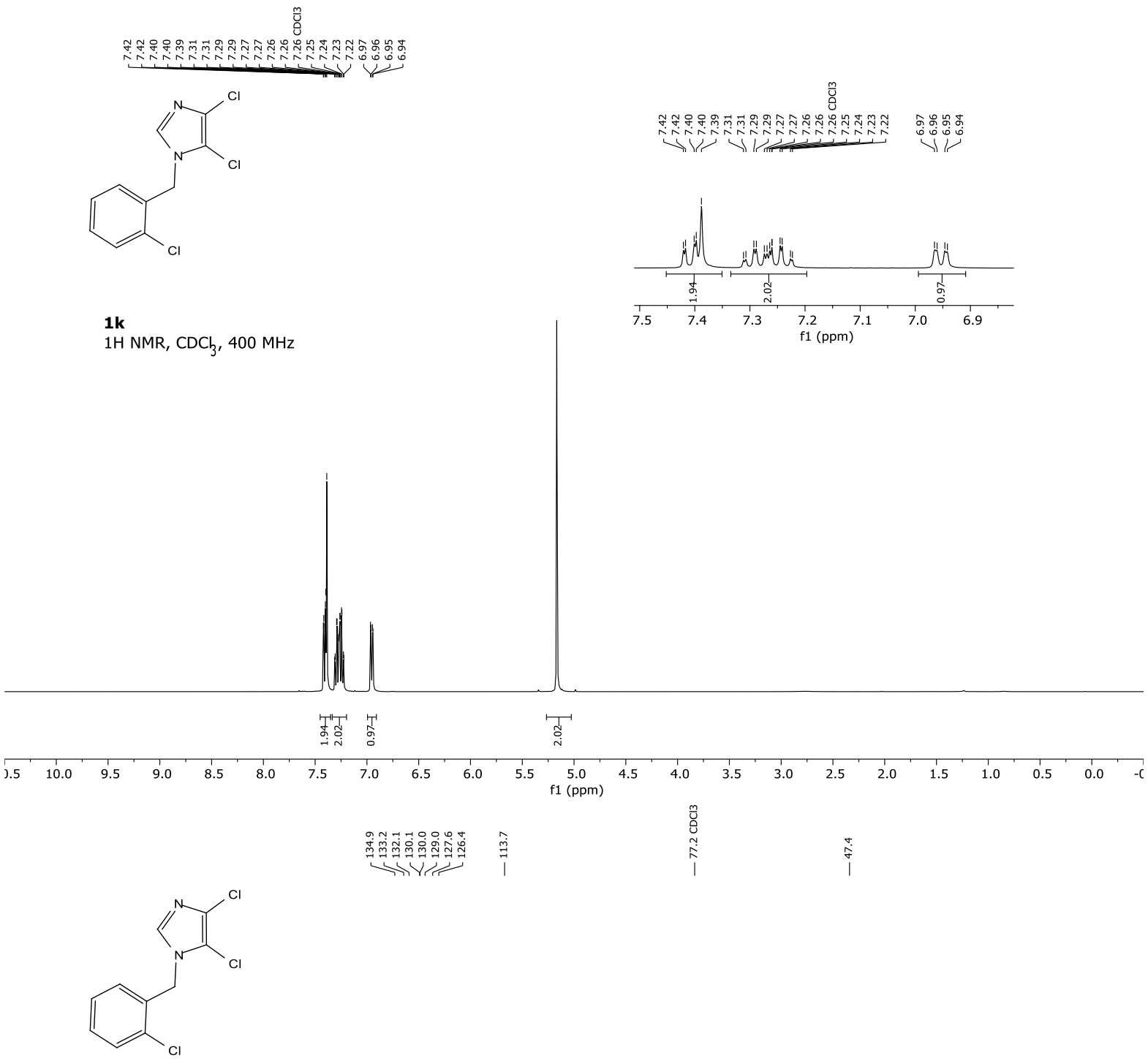

$1 k$

13C NMR, $\mathrm{CDCl}_{3}, 101 \mathrm{MHz}$

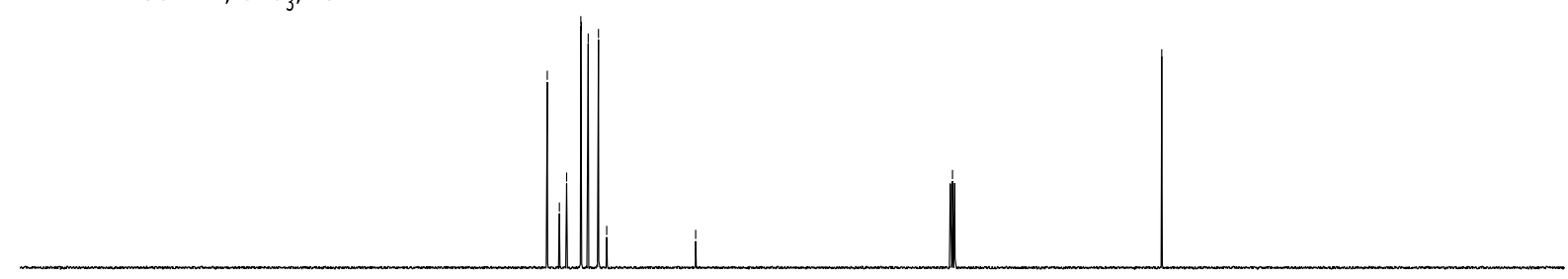

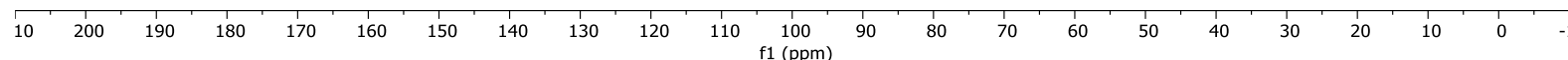


1-(4-Bromobenzyl)-4,5-dichloro-1H-imidazole (11)
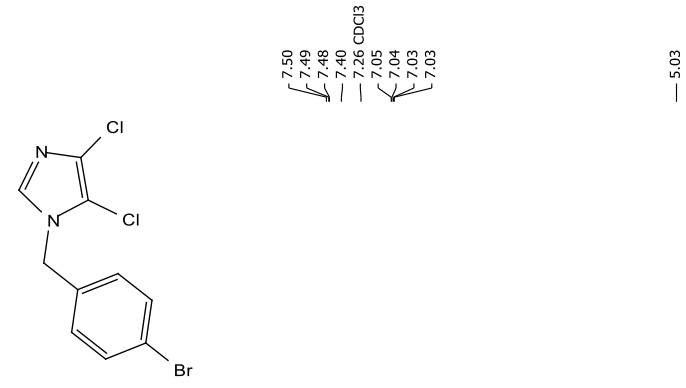

$\stackrel{\substack{0 \\ i}}{i}$

$1 \mathrm{H} \mathrm{NMR,} \mathrm{CDCl}_{3}, 400 \mathrm{MHz}$

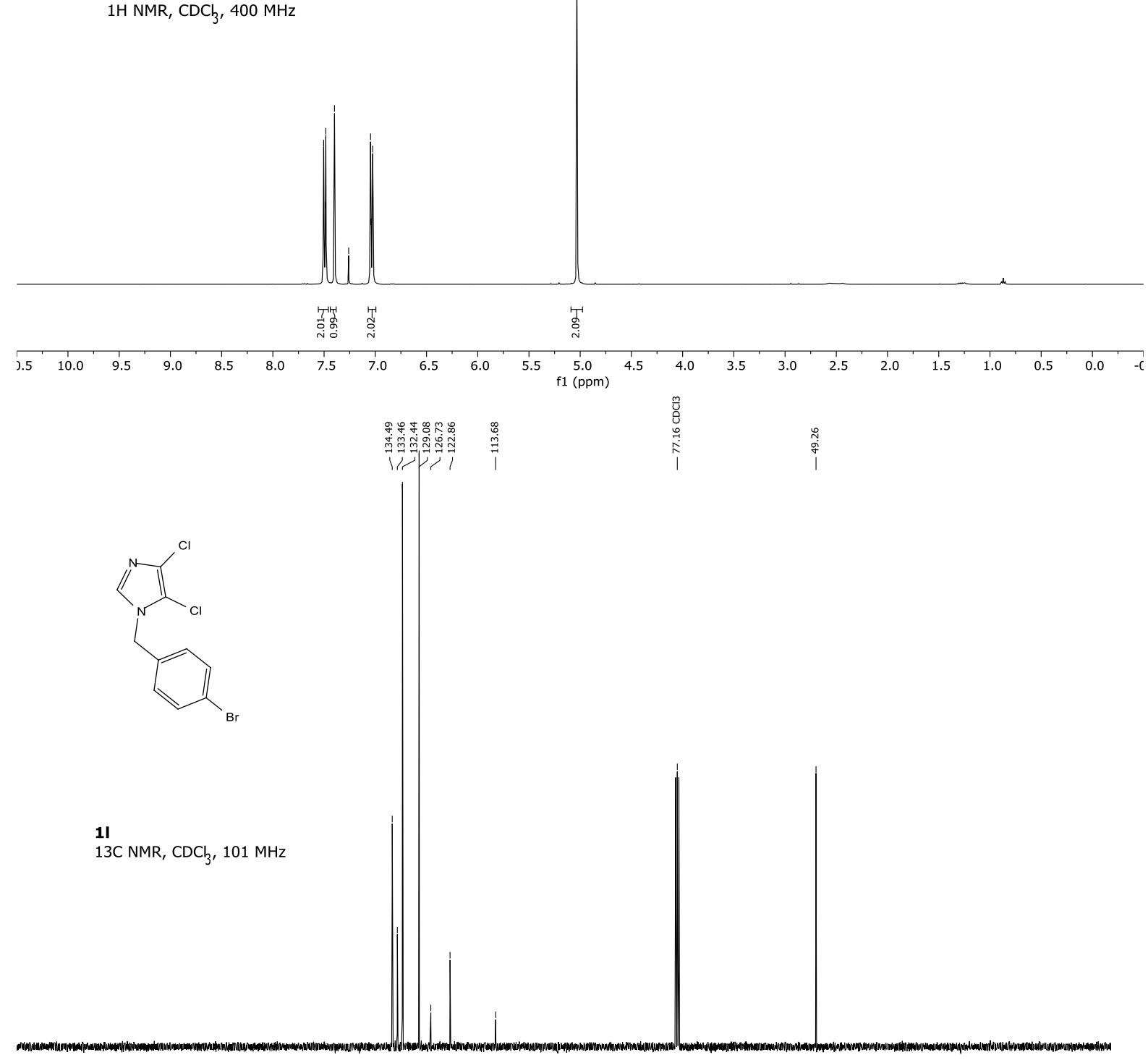

$\begin{array}{llllllllllllllllllllllllll}10 & 200 & 190 & 180 & 170 & 160 & 150 & 140 & 130 & 120 & 110 & 100 & 90 & 80 & 70 & 60 & 50 & 40 & 30 & 20 & 10 & 0 & \\ \mathrm{f} 1(\mathrm{ppm}) & \end{array}$ 
6-Chloro-N-(2-(4,5-dichloro-1H-imidazol-1-yl)ethyl)nicotinamide (1m)

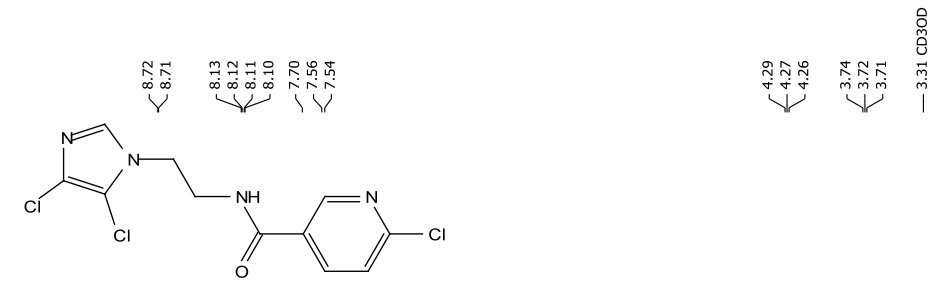

$1 \mathbf{m}$

$1 \mathrm{H} N M R, \mathrm{CD}_{3} \mathrm{OD}, 400 \mathrm{MHz}$
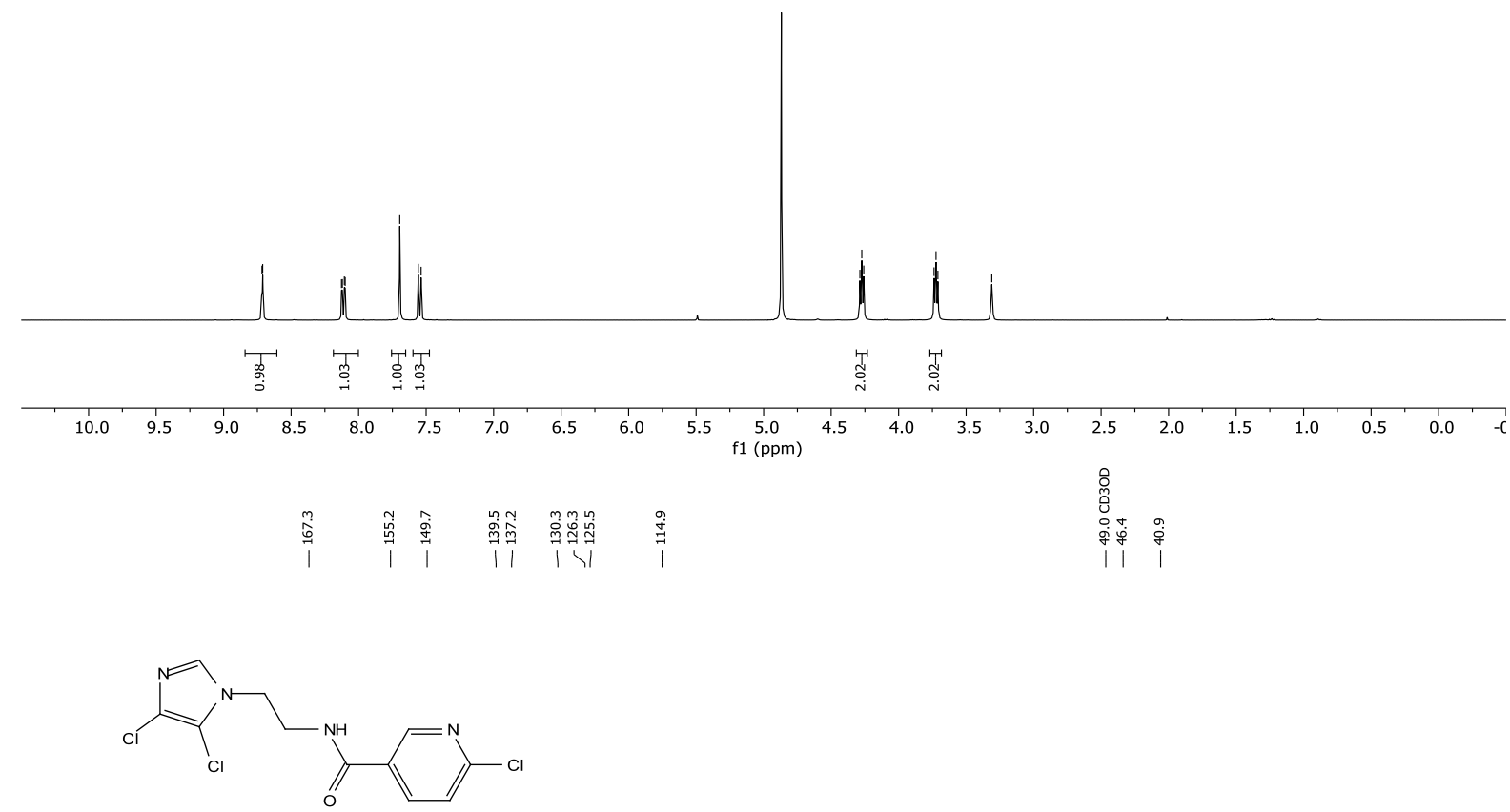

$1 \mathrm{~m}$

$13 \mathrm{C} N M R, \mathrm{CD}_{3} \mathrm{OD}, 101 \mathrm{MHz}$

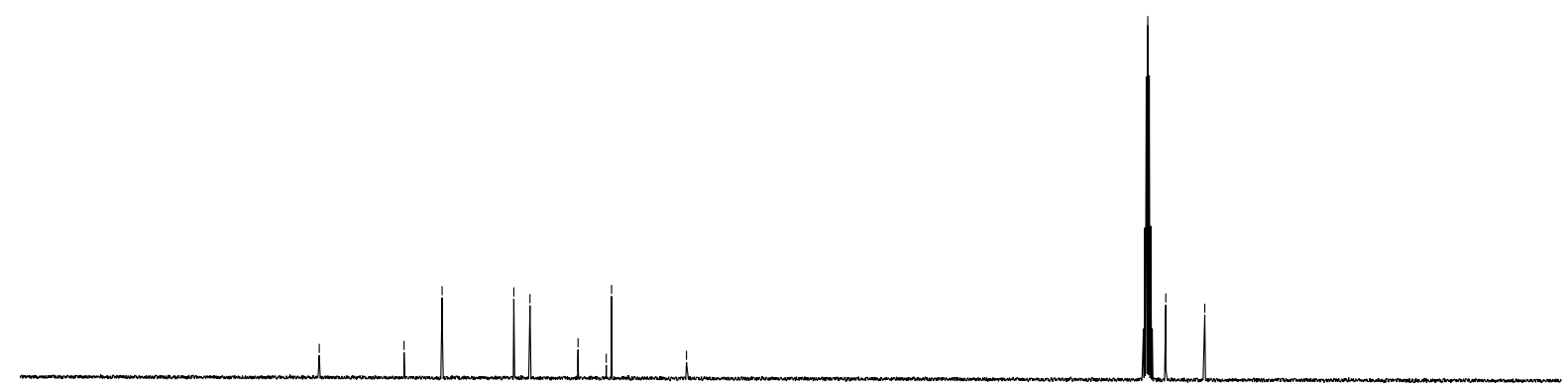

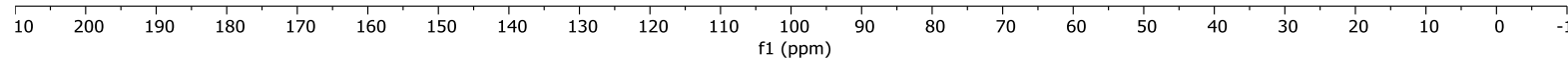


2-Bromo-5-phenylfuran (1p)

范

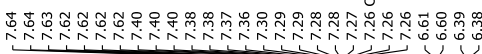<smiles>Brc1ccc(-c2ccccc2)o1</smiles>

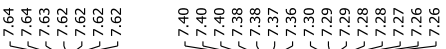

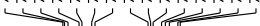

1p

$1 \mathrm{H} N \mathrm{NR}, \mathrm{CDCl}_{3}, 400 \mathrm{MHz}$
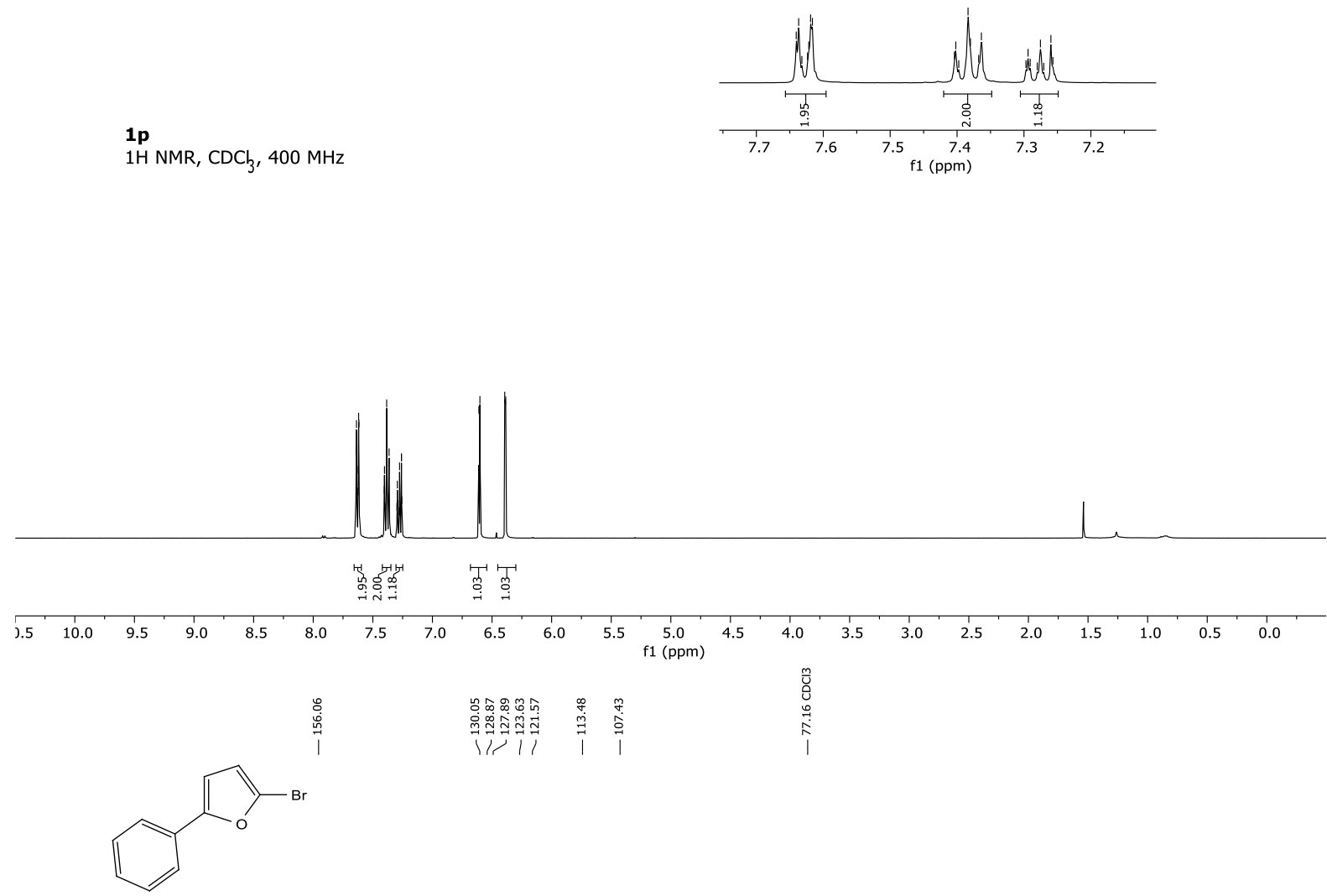

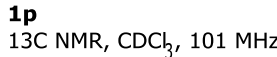

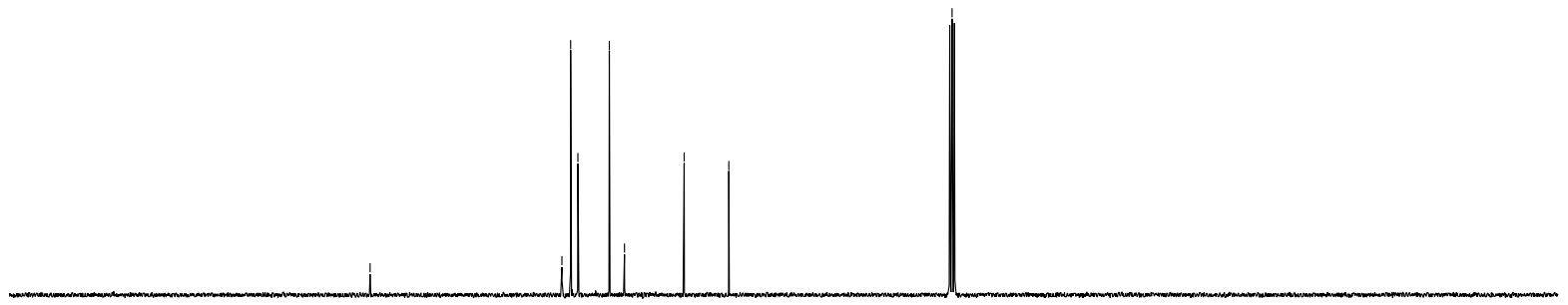

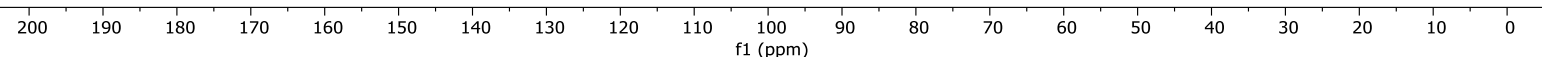




\section{2,4-Dibromo-5-phenylfuran (1q)}

范

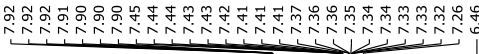<smiles>Brc1cc(Br)c(-c2ccccc2)o1</smiles>

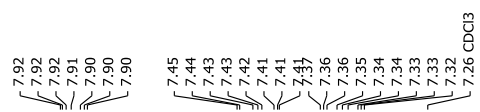

$1 \mathrm{H} N \mathrm{NR}, \mathrm{CDCl}_{3}, 400 \mathrm{MHz}$
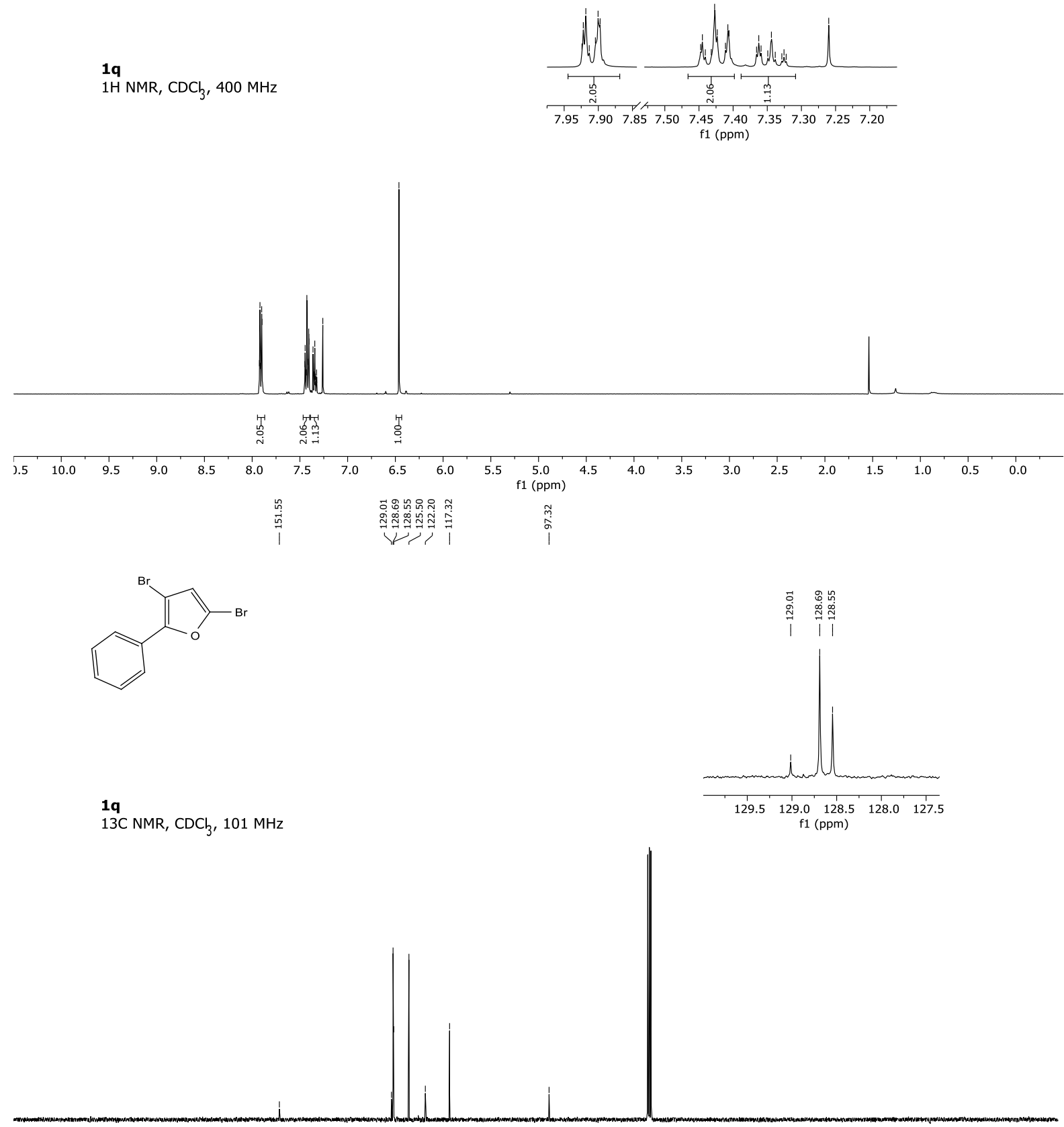

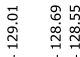

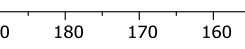

150

$140 \quad 130$

120

100
$\mathrm{f} 1(\mathrm{ppm})$ 
2-Chloro-1-methyl-5-phenyl-1H-pyrrole (1r)

兽

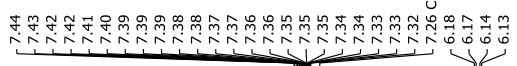<smiles>Cn1c(Cl)ccc1-c1ccccc1</smiles>

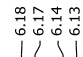

1r

$1 \mathrm{H} \mathrm{NMR}, \mathrm{CDCl}_{3}, 300 \mathrm{MHz}$

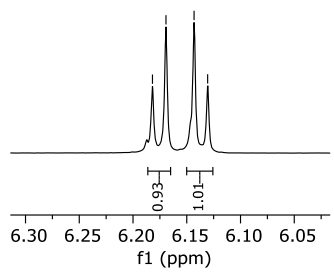

f1 (ppm)
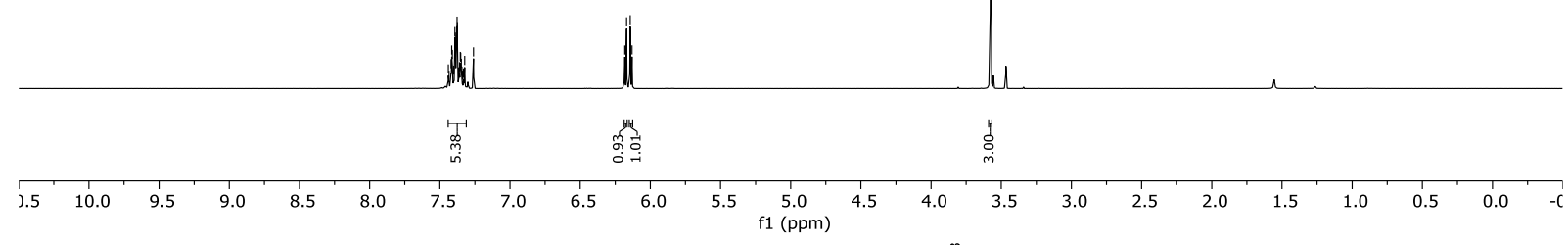

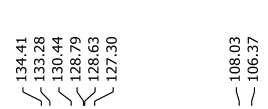<smiles>Cn1c(Cl)ccc1-c1ccccc1</smiles>

1r

13C NMR, $\mathrm{CDCl}_{3}, 101 \mathrm{MHz}$

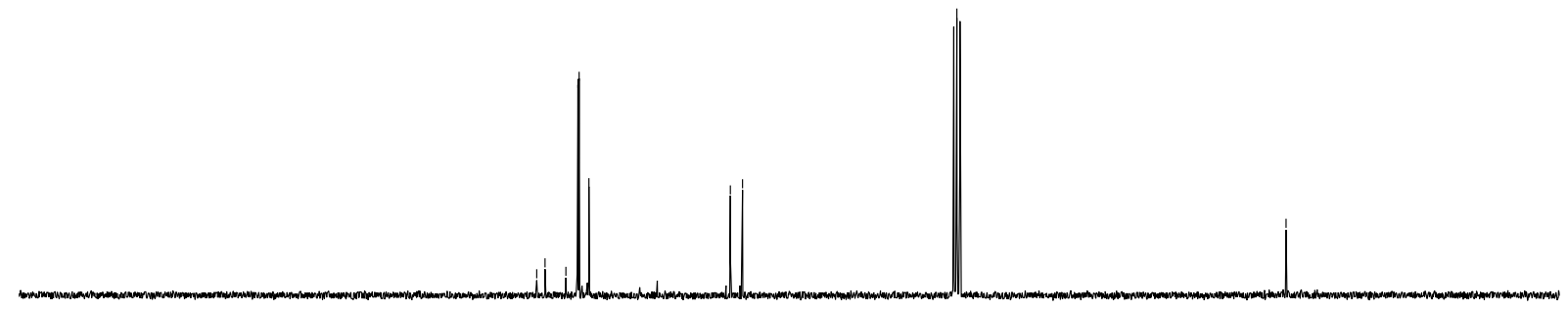


2,3,4-Trichloro-1-methyl-5-phenyl-1H-pyrrole (1s)

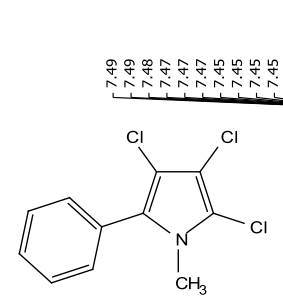
$1 \mathrm{H} \mathrm{NMR,} \mathrm{CDCl}_{3}, 400 \mathrm{MHz}$
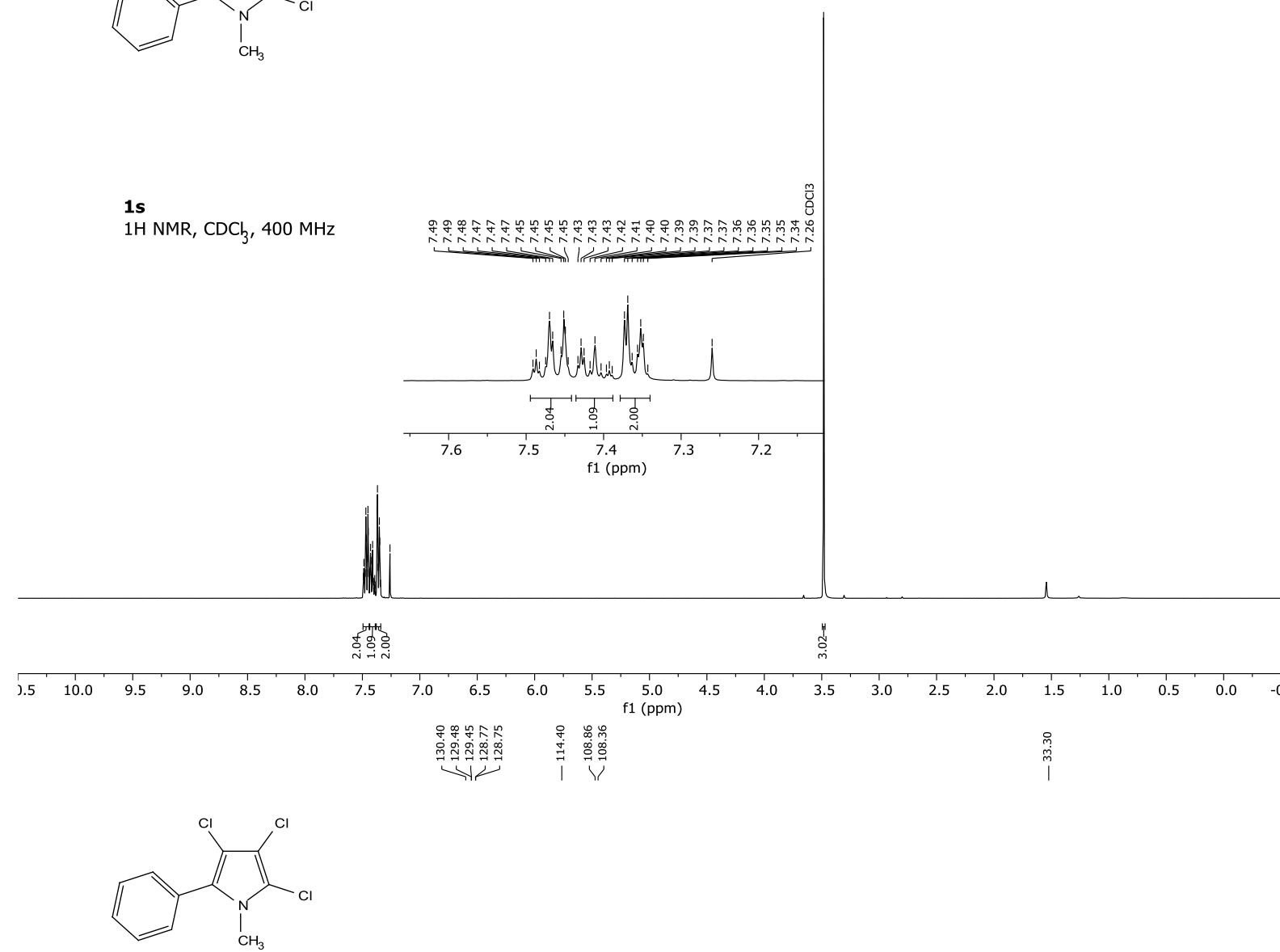

1s $13 \mathrm{C} \mathrm{NMR,} \mathrm{CDCl}_{3}, 101 \mathrm{MHz}$

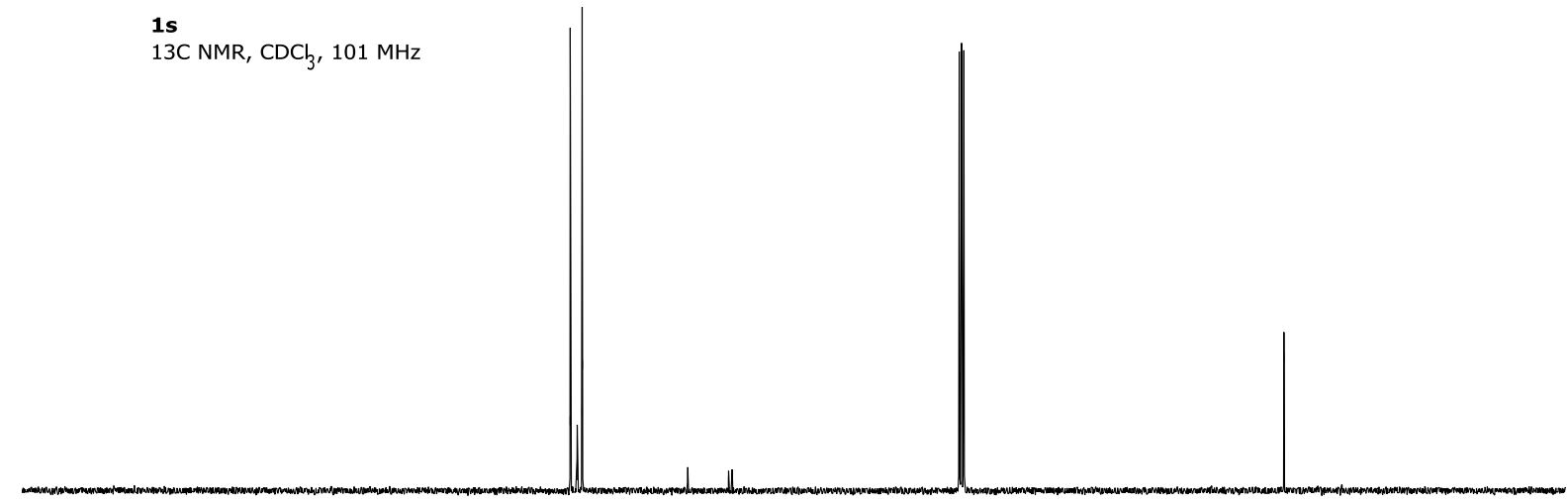

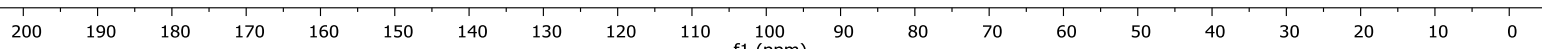


2,3,4-Tribromo-1-methyl-5-phenyl- $1 H$-pyrrole (1t)

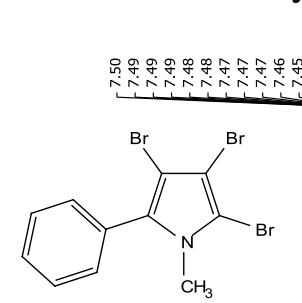

1t

$1 \mathrm{H} \mathrm{NMR}, \mathrm{CDCl}_{3}, 300 \mathrm{MHz}$

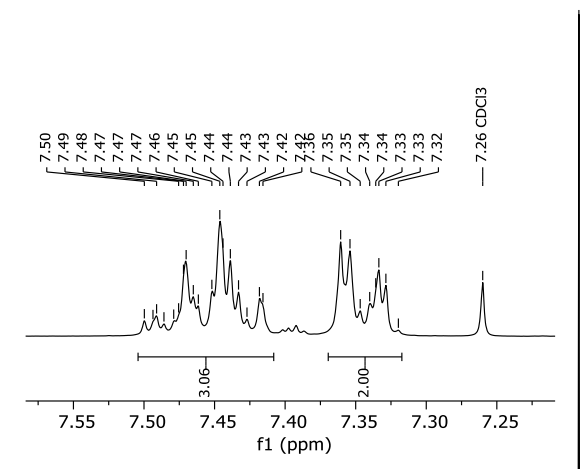

in
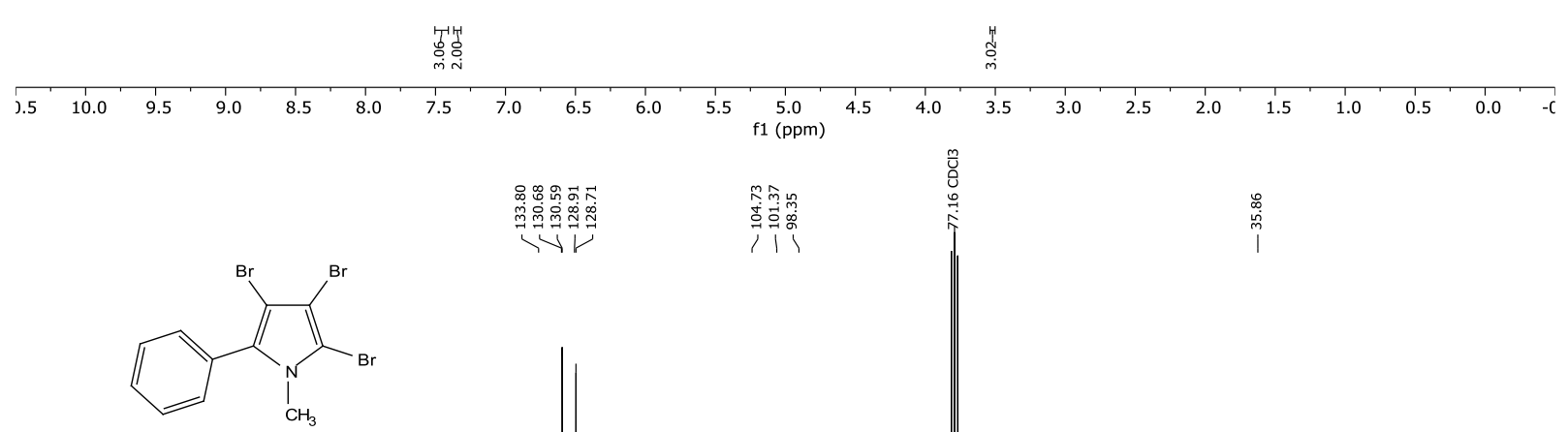

1t
$13 \mathrm{C} \mathrm{NMR,} \mathrm{CDCl}_{3}, 75 \mathrm{MHz}$

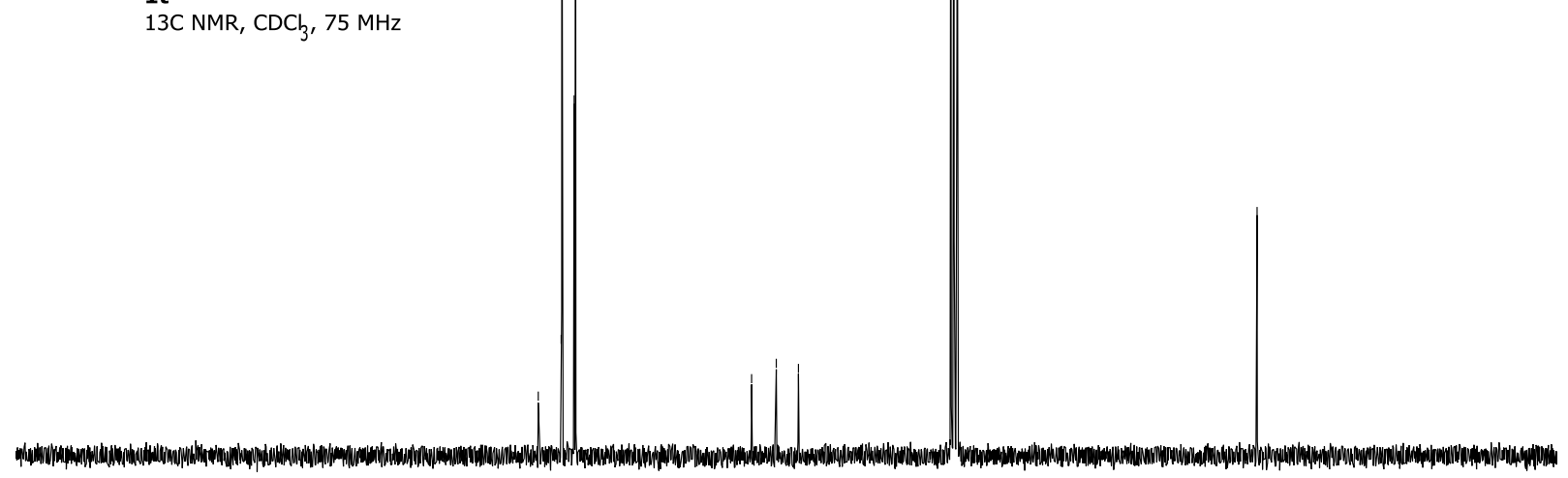

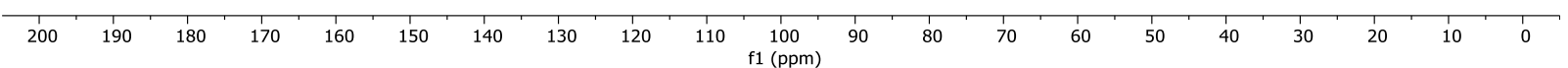


5-Chloro-4-phenylthiazol-2-amine (1ao)
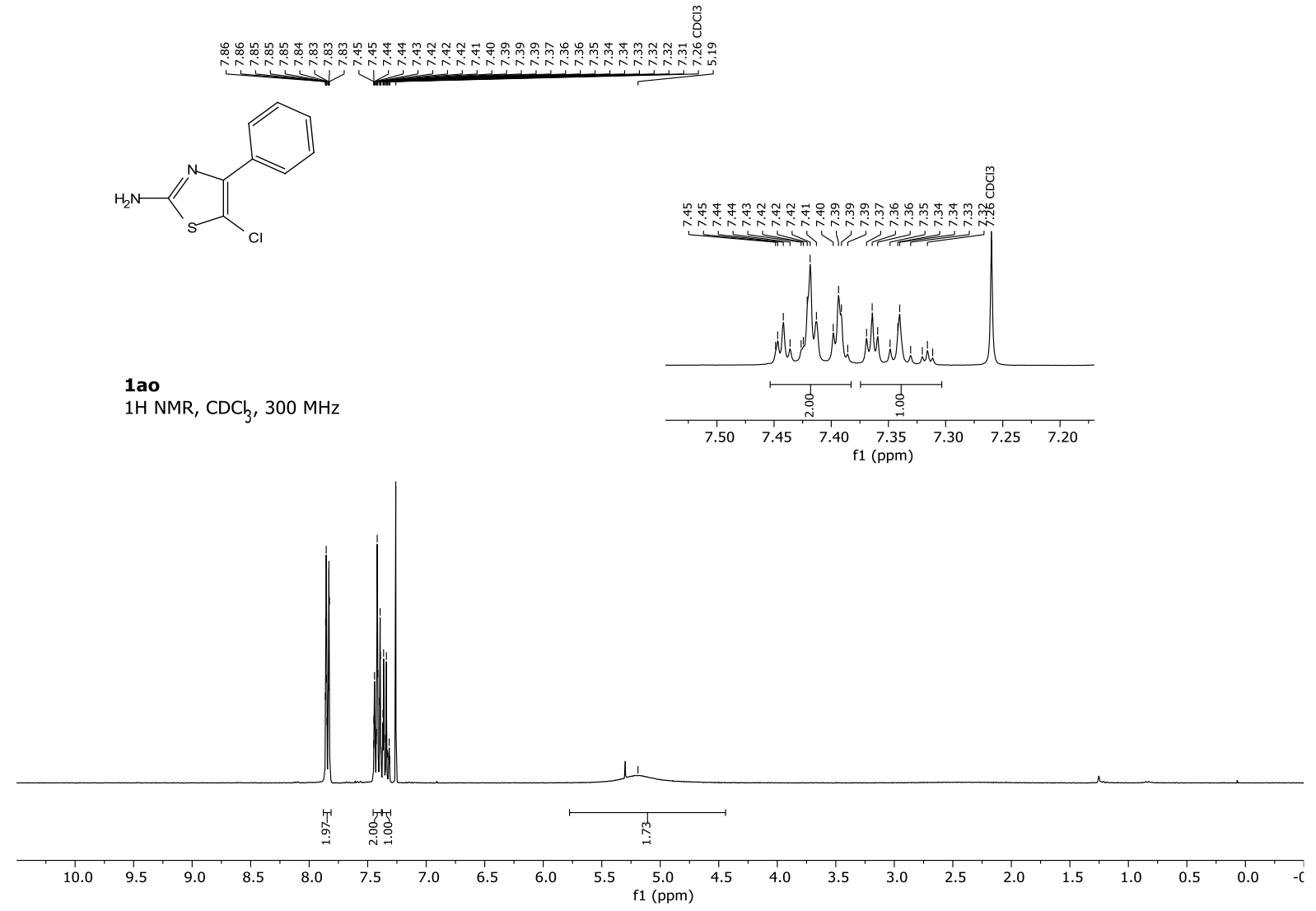

${ }_{13 \mathrm{C}} \mathrm{NMR}, \mathrm{CDCl}_{3}, 75 \mathrm{MHz}$

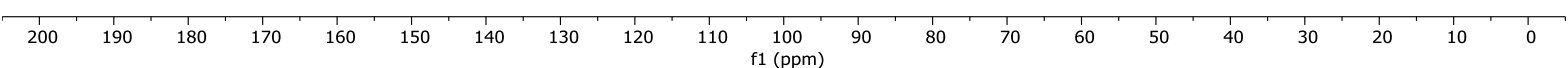


2,2,2-Trifluoro- $N$-(4-mercaptophenyl)acetamide (2ai)

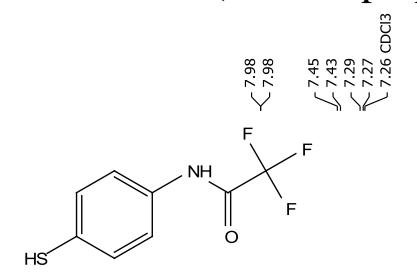

2ai

$1 \mathrm{H} \mathrm{NMR}, \mathrm{CDCl}_{3}, 400 \mathrm{MHz}$

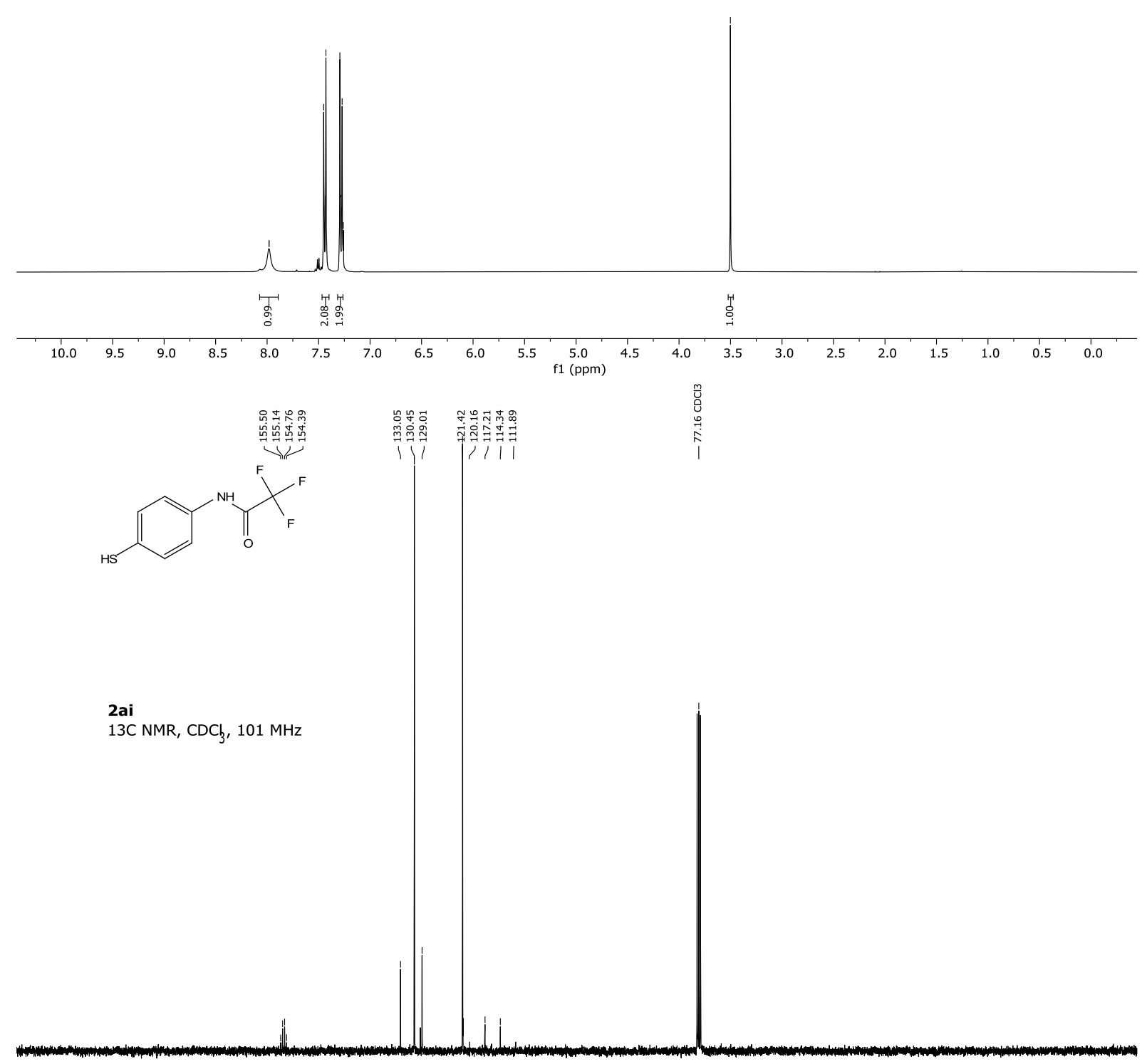

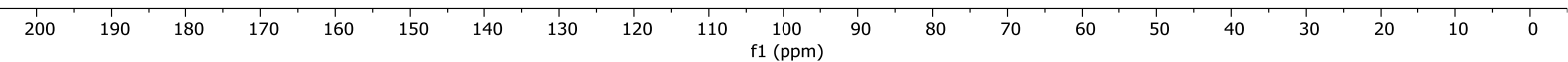




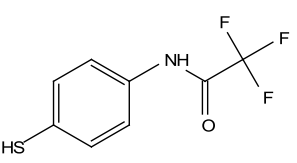

2ai

19F NMR, $\mathrm{CDCl}_{3}, 376 \mathrm{MHz}$

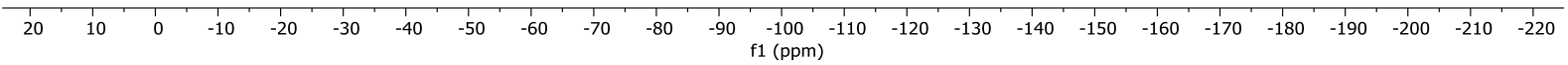


$N$-(2-mercaptoethyl)-5-((3aS,4S,6aR)-2-oxohexahydro-1H-thieno[3,4-d]imidazol-4yl)pentanamide (2aj)

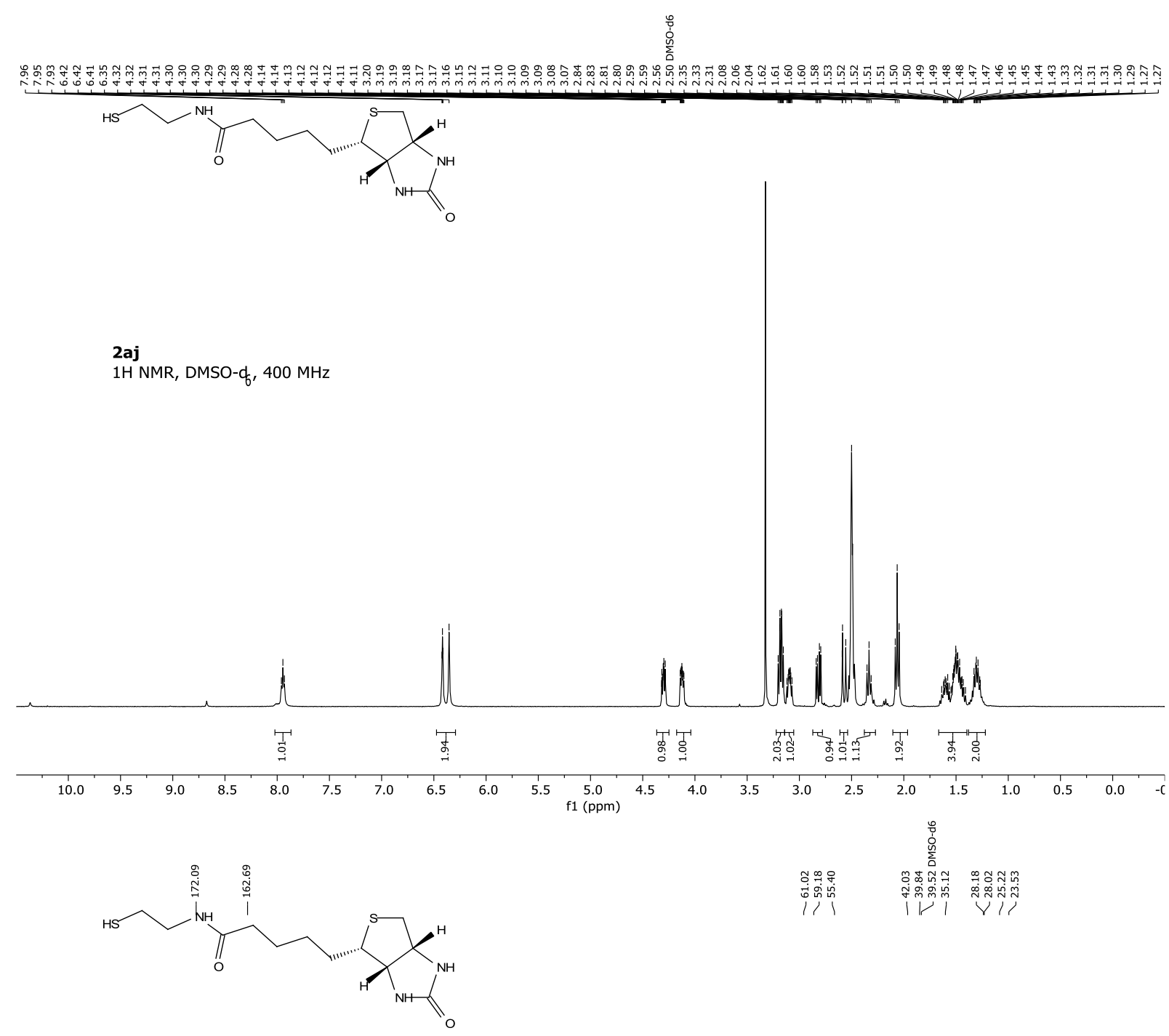

2aj
$13 \mathrm{C}$ NMR, DMSO-q, $101 \mathrm{MHz}$

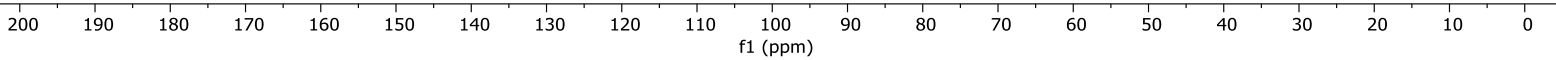


$(3 R, 5 S, 8 R, 9 S, 10 S, 13 R, 14 S, 17 R)-10,13-D i m e t h y l-17-((\mathrm{R})-6-m e t h y l h e p t a n-2-$ yl)hexadecahydro-1H-cyclopenta[a]phenanthrene-3-thiol (2al) 总

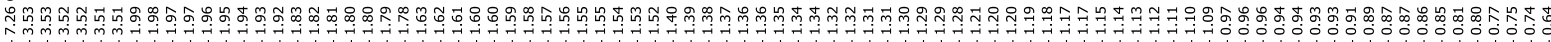
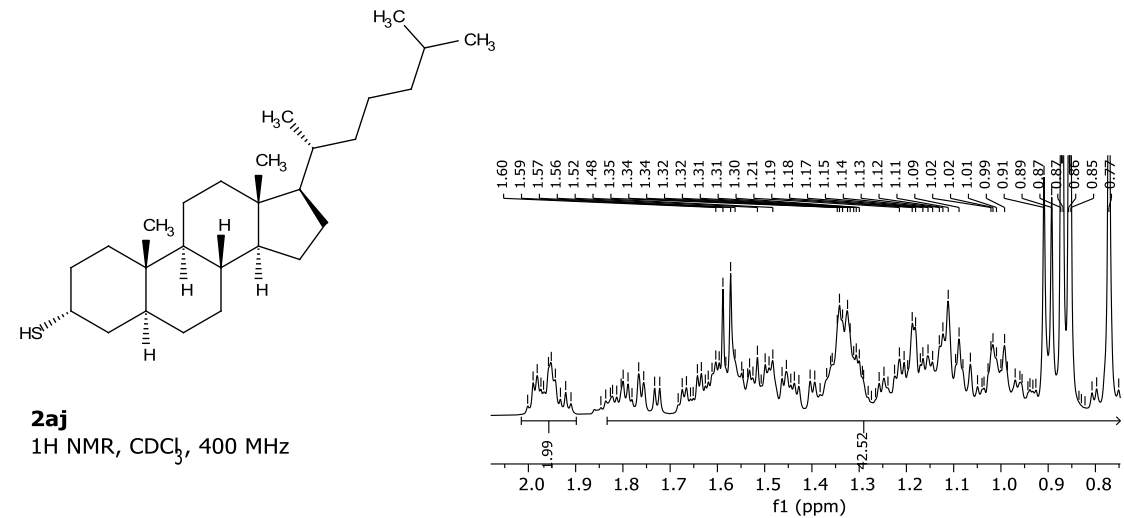

2aj

$1 \mathrm{H} \mathrm{NMR}, \mathrm{CDCl}_{3}, 400 \mathrm{MHz}$
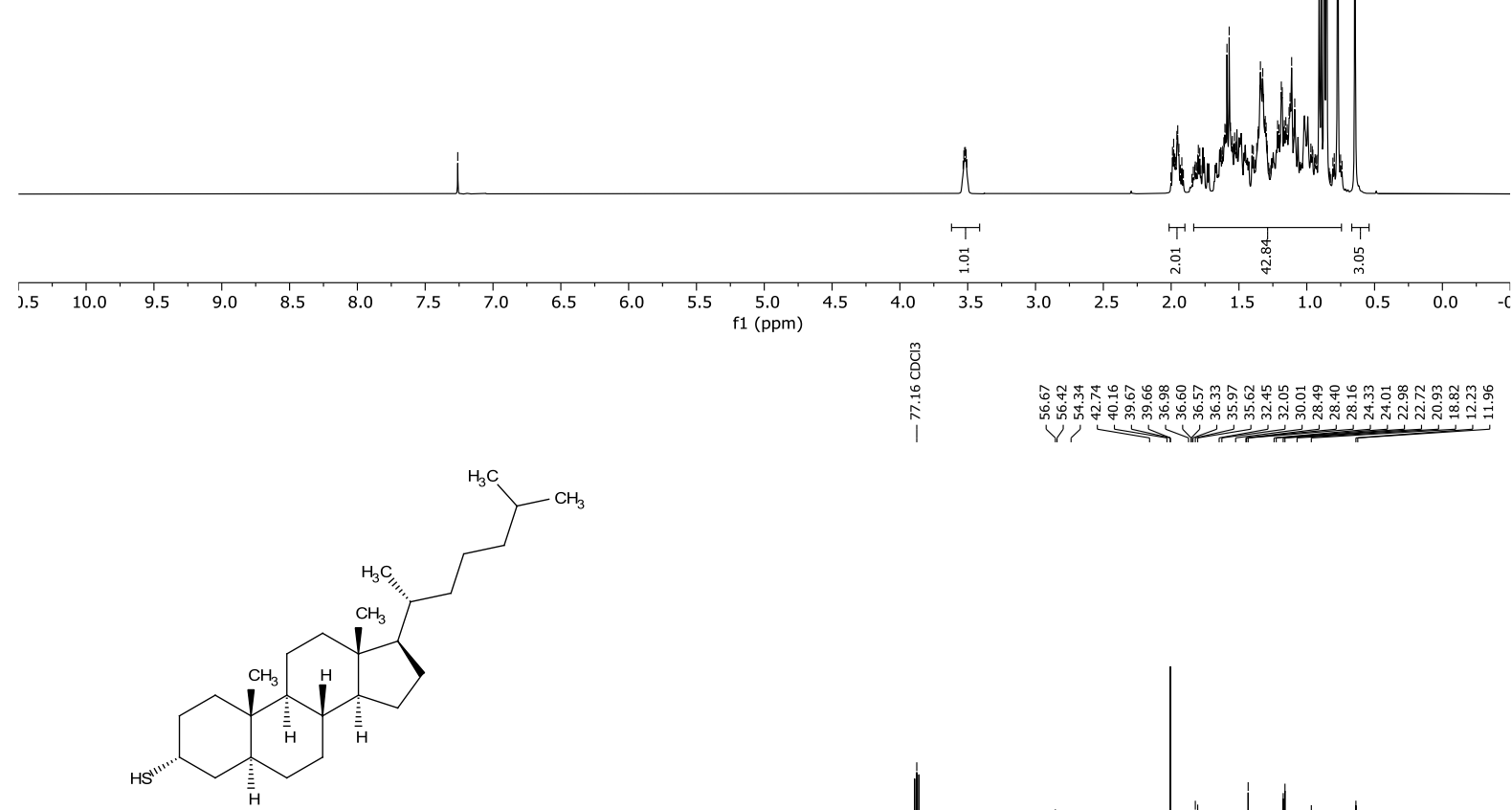

2al

13C NMR, $\mathrm{CDCl}_{3}, 101 \mathrm{MHz}$

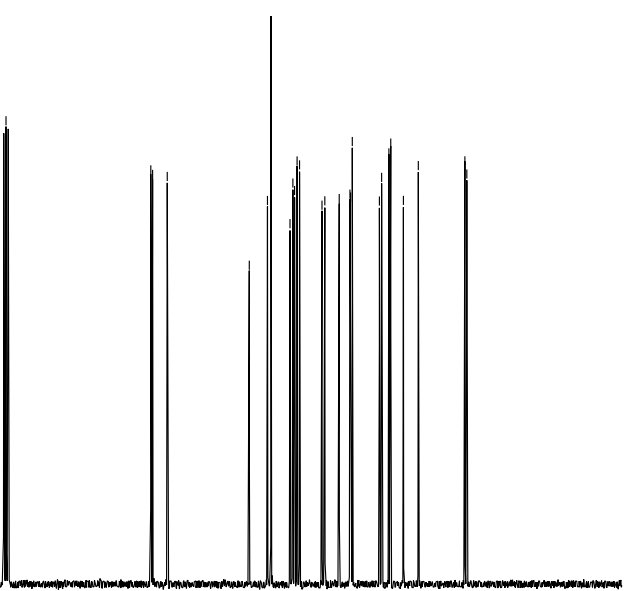

10

$200 \quad 190$

$190 \quad 180 \quad 170 \quad 160$

$150 \quad 140$

$130 \quad 12$ $10 \begin{gathered}100 \\ \mathrm{f} 1(\mathrm{ppm})\end{gathered}$ 
Methyl $\quad N^{2}$-(tert-butoxycarbonyl)- $N^{4}$-(2-mercaptoethyl)- $L$-asparaginyl- $L$-phenylalaninate (2am)

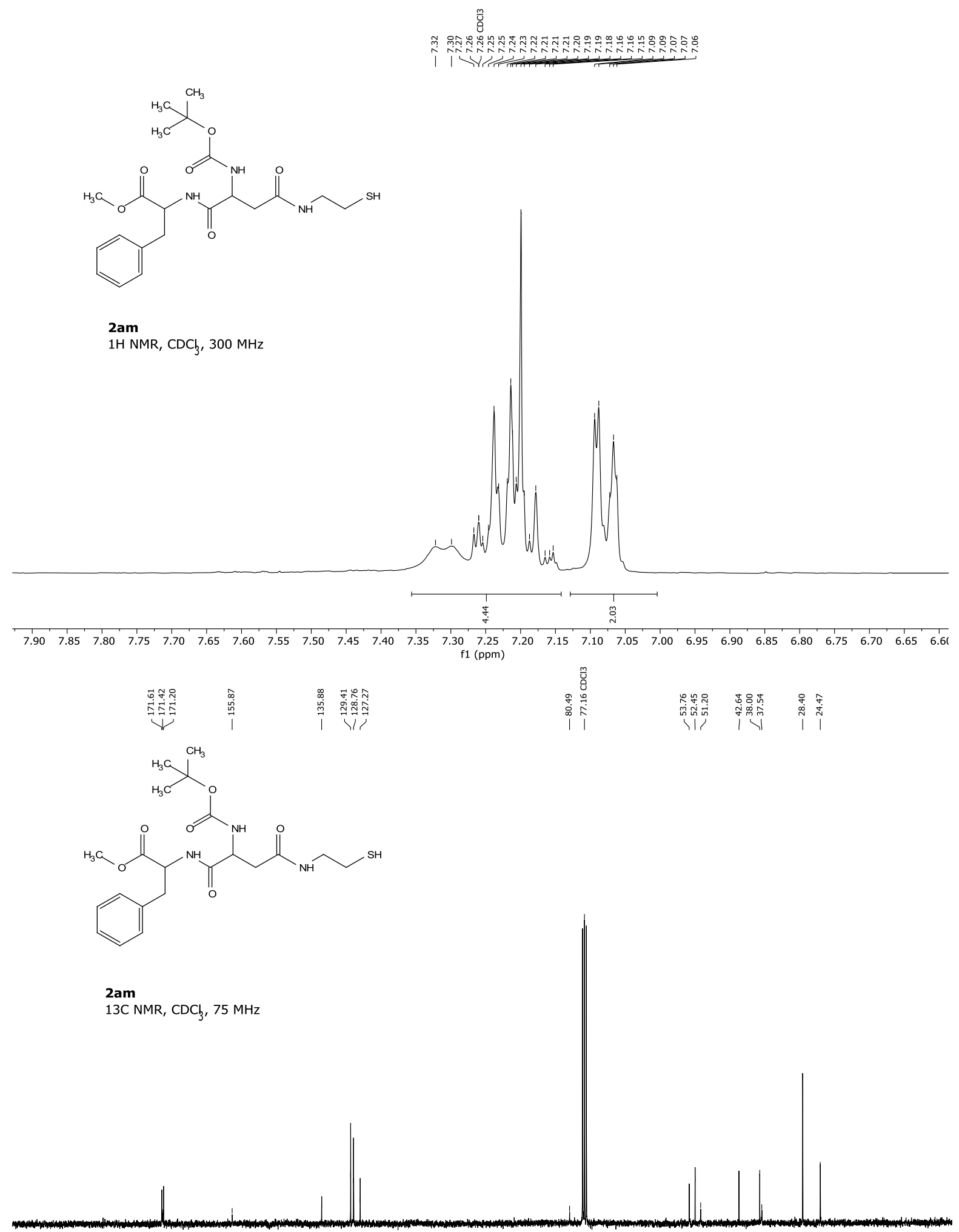

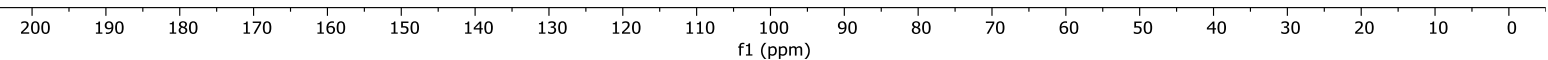


Methyl 3-((4-chloro-1-methyl-1H-imidazol-5-yl)thio)propanoate (3a)
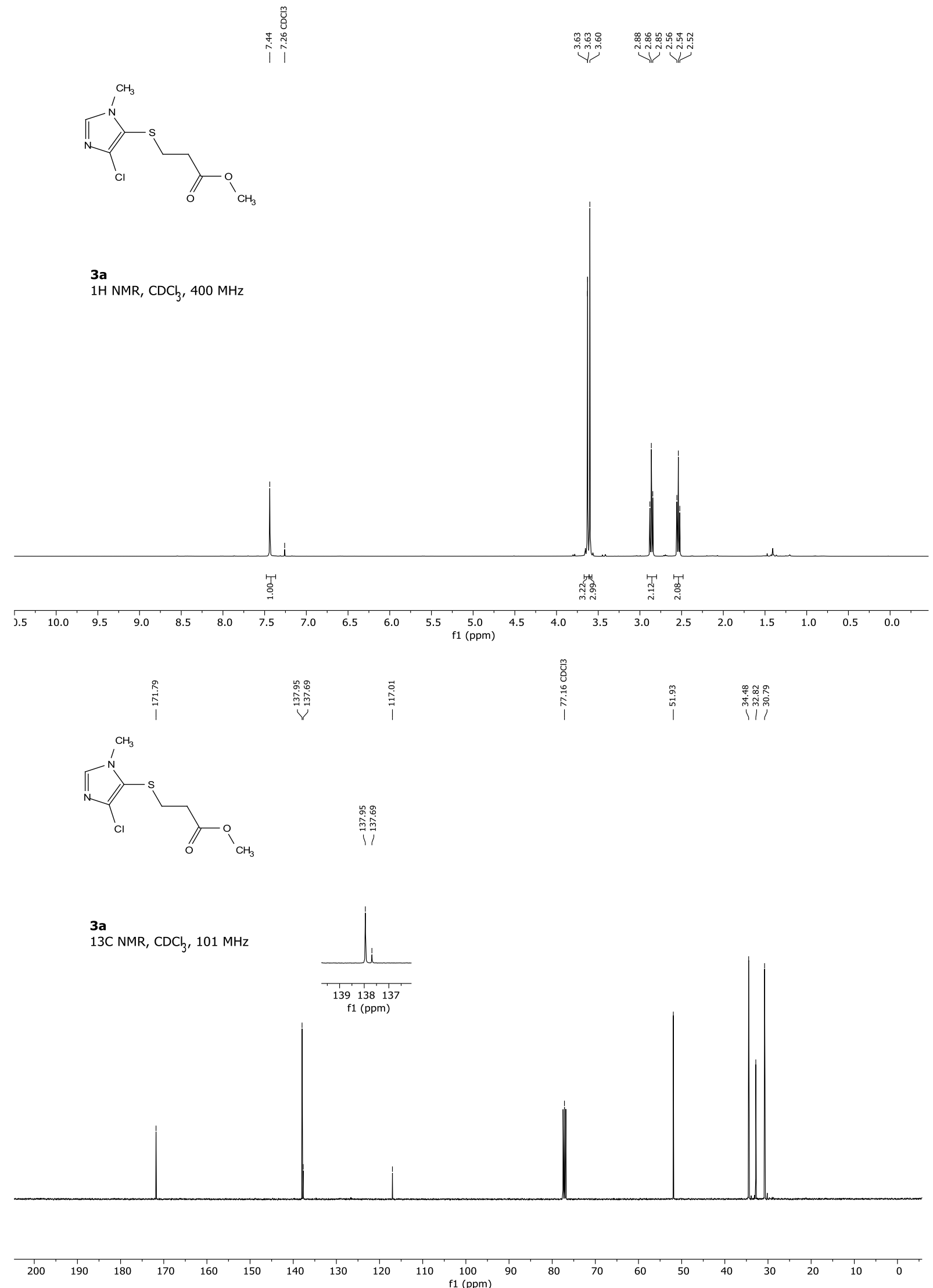
Methyl 3-((4-bromo-1-methyl-1H-imidazol-5-yl)thio)propanoate (3b)<smiles>COC(=O)CCSc1c(Br)ncn1O</smiles>

$1 \mathrm{H} \mathrm{NMR,} \mathrm{CDCl}_{3}, 400 \mathrm{MHz}$

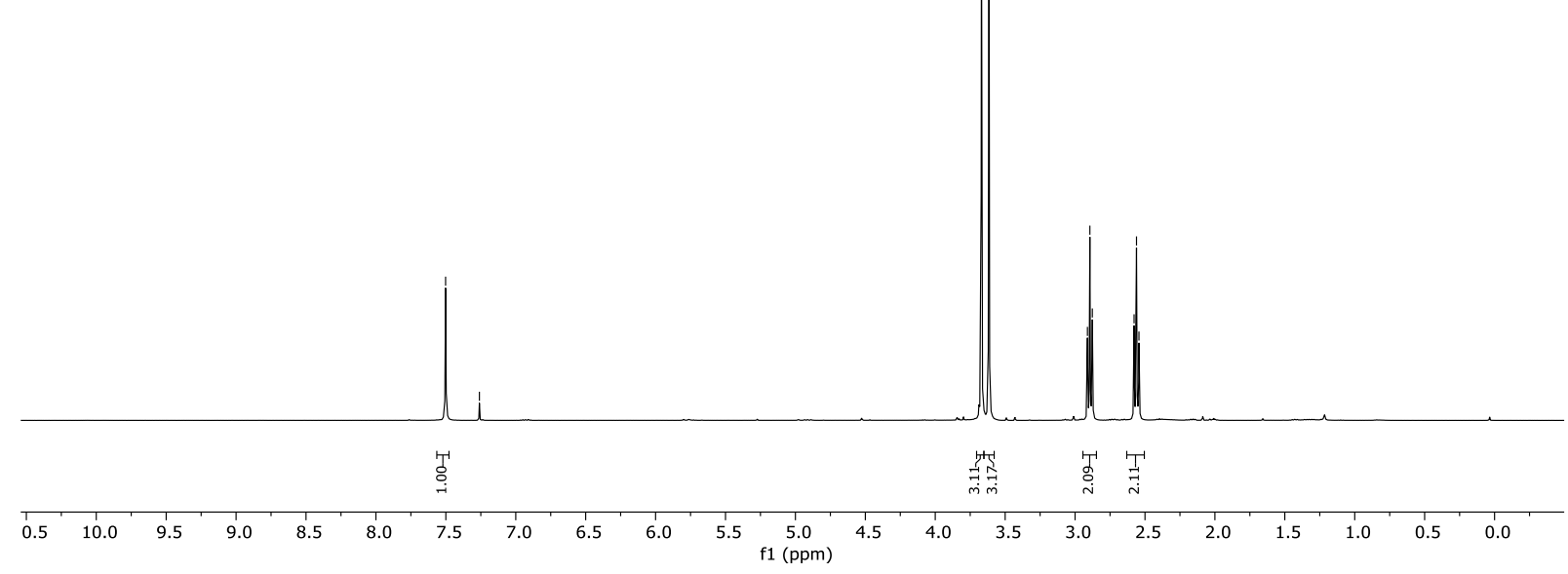<smiles>COC(=O)CCSc1c(Br)ncn1C</smiles>

$3 b$

13C NMR, $\mathrm{CDCl}_{3}, 101 \mathrm{MHz}$

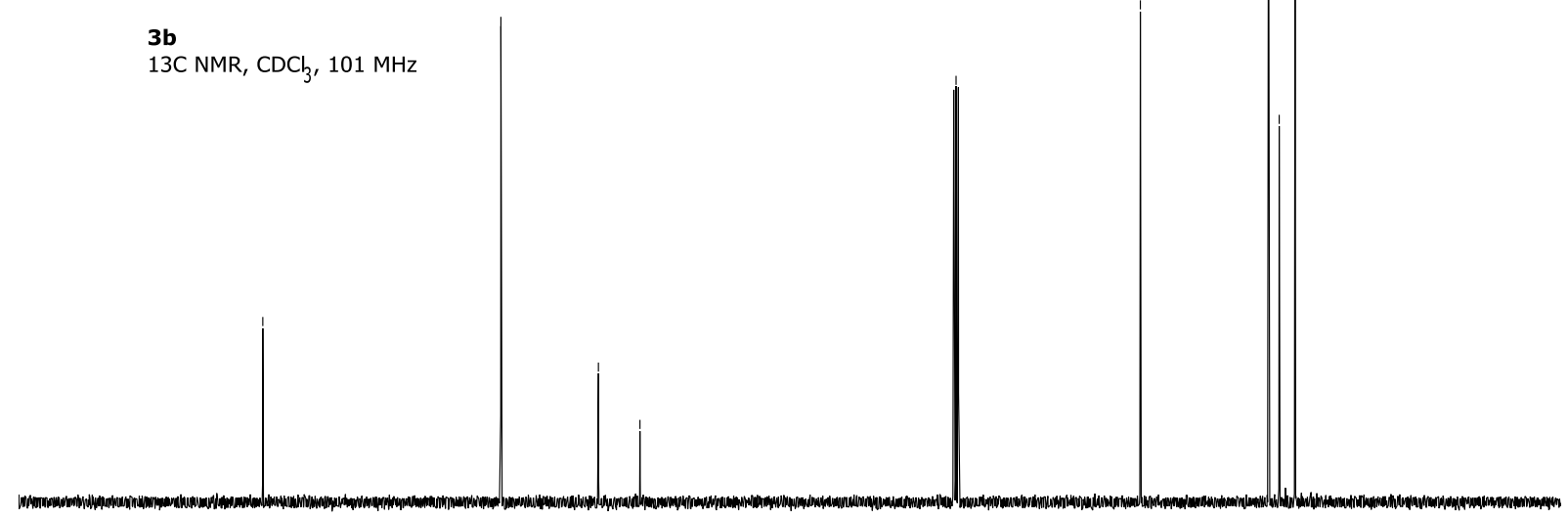

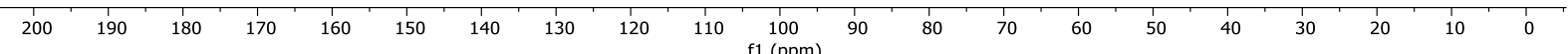


Methyl 3-((1-ethyl-2-methyl-1H-imidazol-5-yl)thio)propanoate (3c)

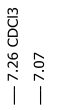

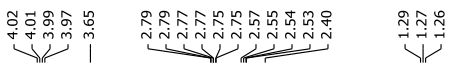

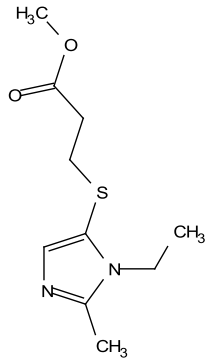

1H NMR, $\mathrm{CDCl}_{3}, 400 \mathrm{MHz}$
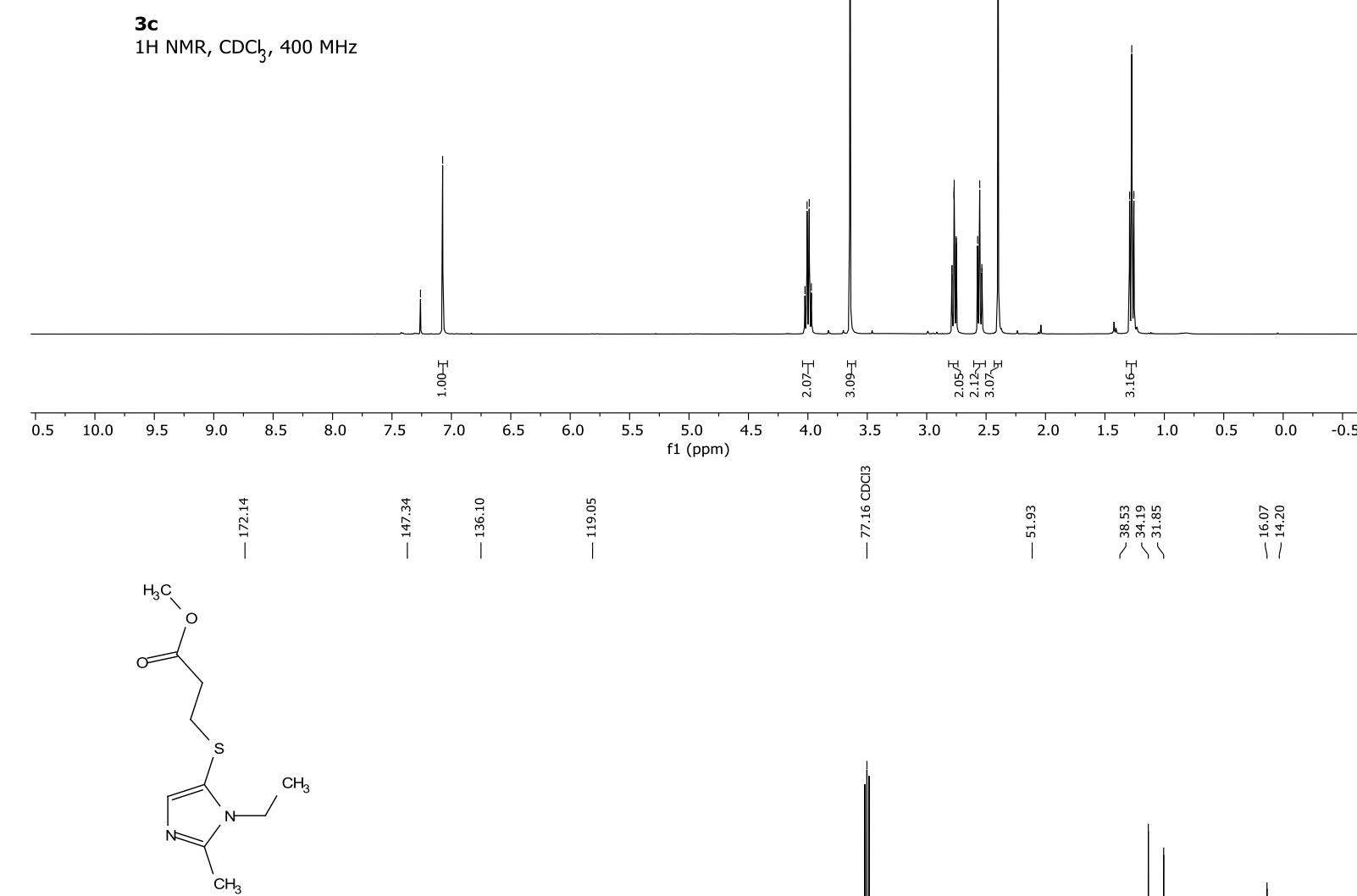

$3 c$

13C NMR, $\mathrm{CDCl}_{3}, 101 \mathrm{MHz}$

200
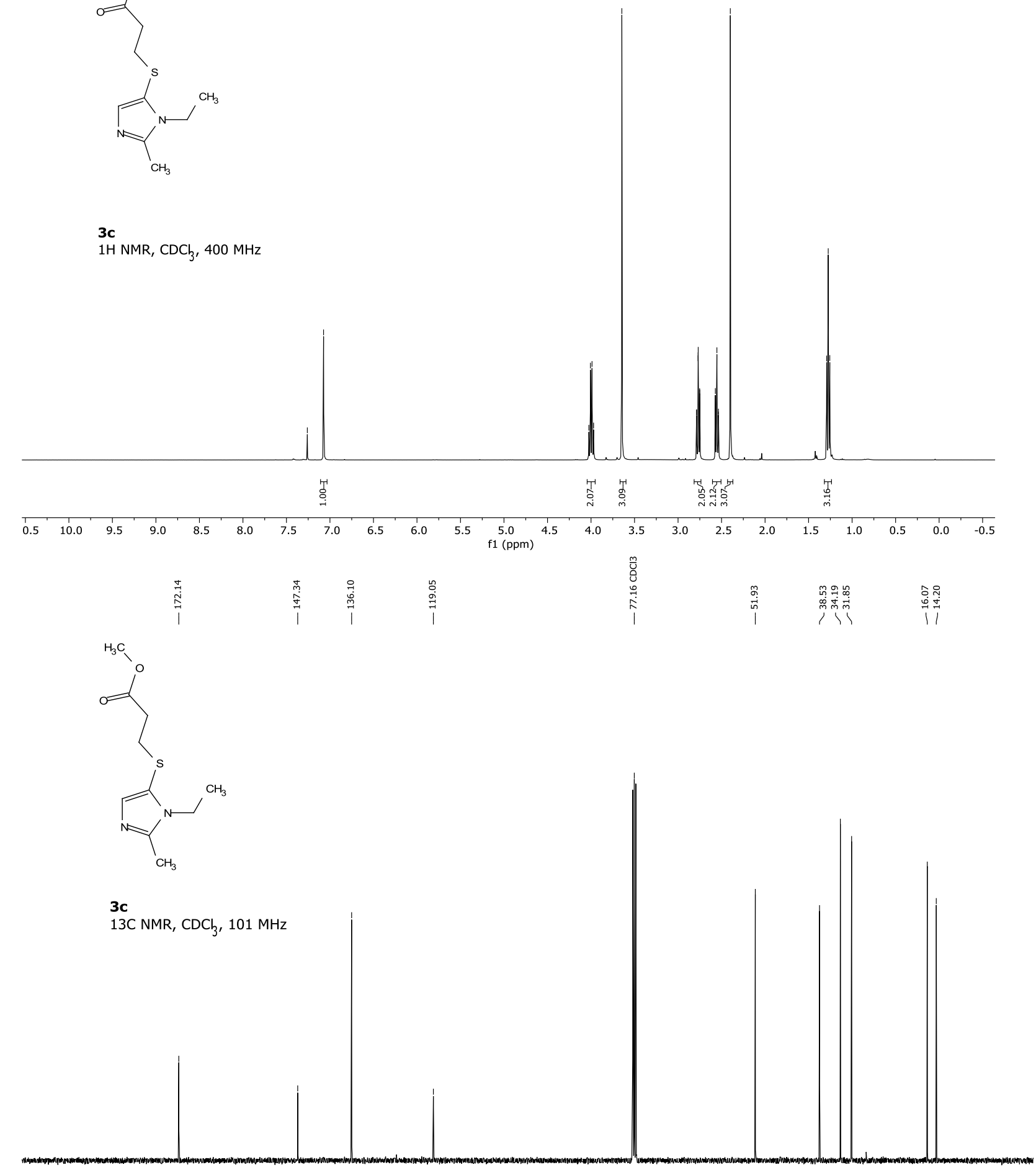
Methyl 3-((1-methyl-1H-imidazol-5-yl)thio)propanoate (3d)

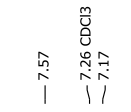

\丶

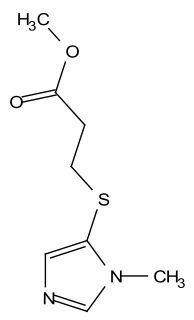

3d

$1 \mathrm{H} N \mathrm{MR}, \mathrm{CDCl}_{3}, 400 \mathrm{MHz}$
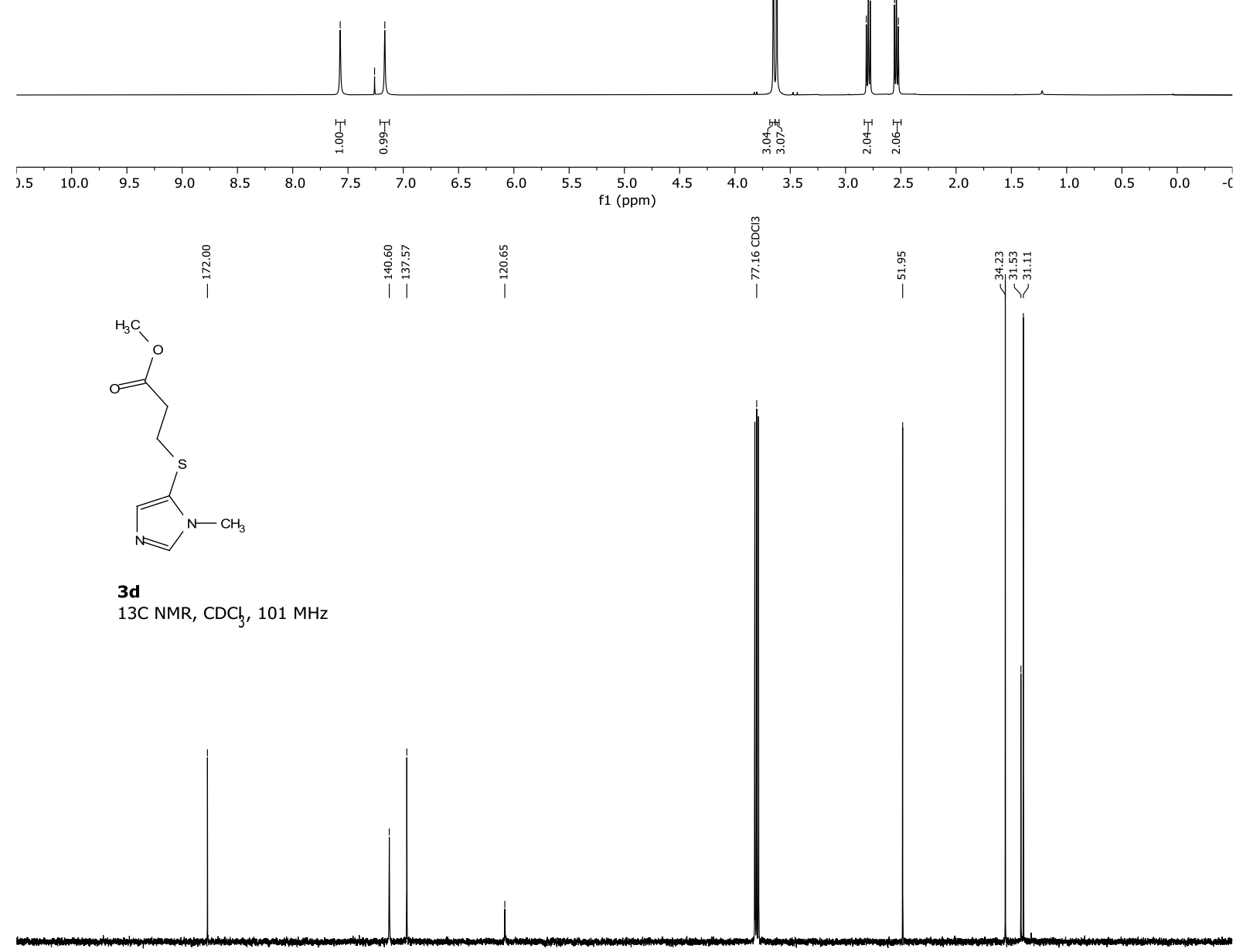

3d

13C NMR, $\mathrm{CDCl}_{3}, 101 \mathrm{MHz}$

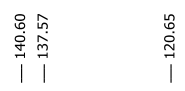

$m$
0
0
0
0
$i$
$i$

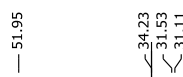

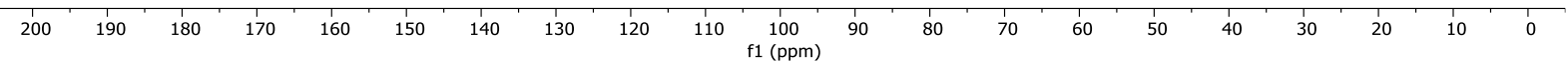


Methyl 3-((1,4-dimethyl-1H-imidazol-2-yl)thio)propanoate (3e)

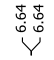

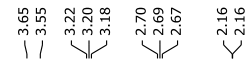

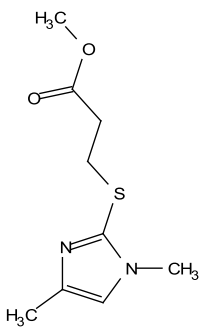

3e

$1 \mathrm{H} \mathrm{NMR}, \mathrm{CDCl}_{3}, 400 \mathrm{MHz}$
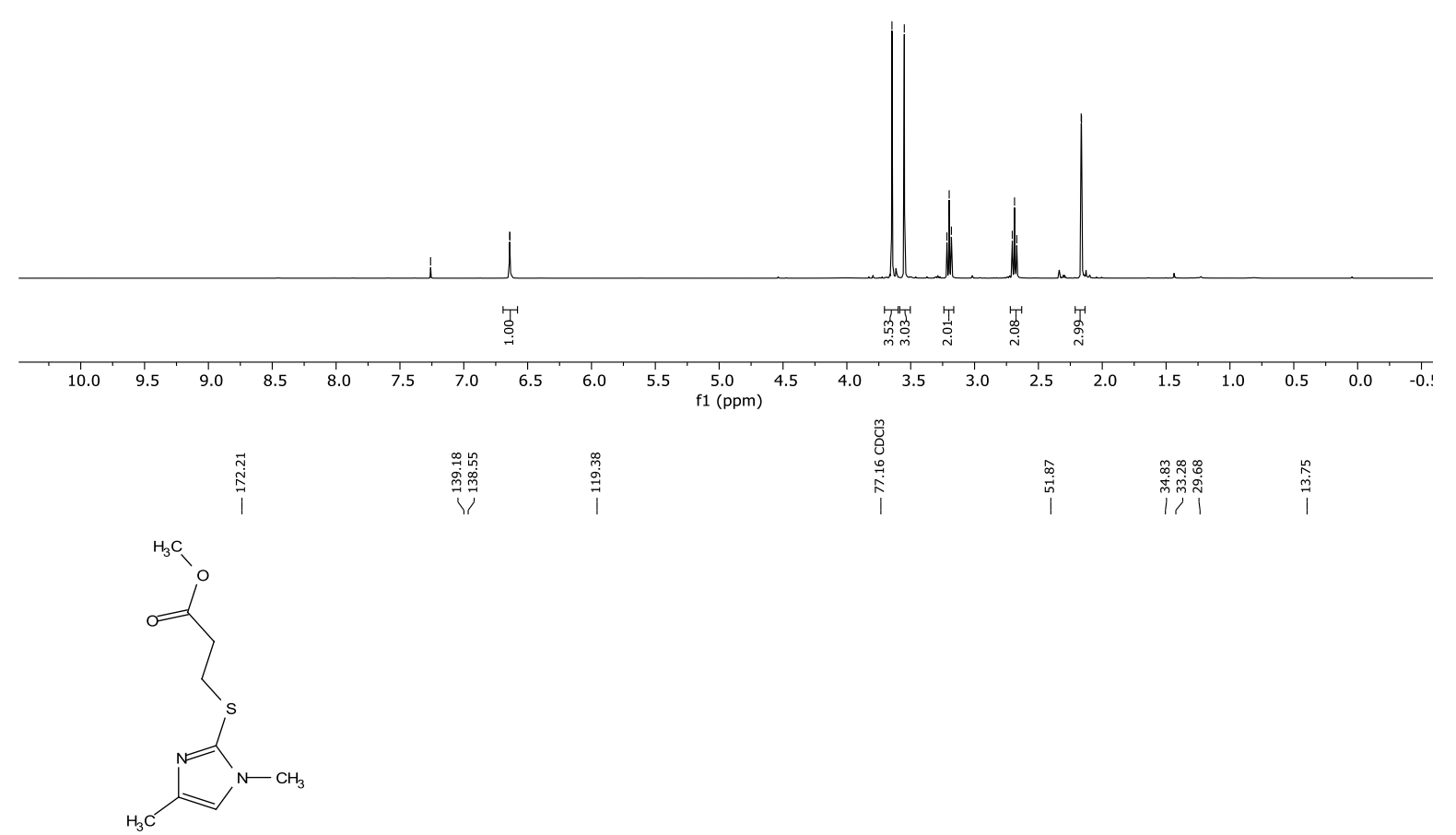

$3 e$

13C NMR, $\mathrm{CDCl}_{3}, 101 \mathrm{MHz}$

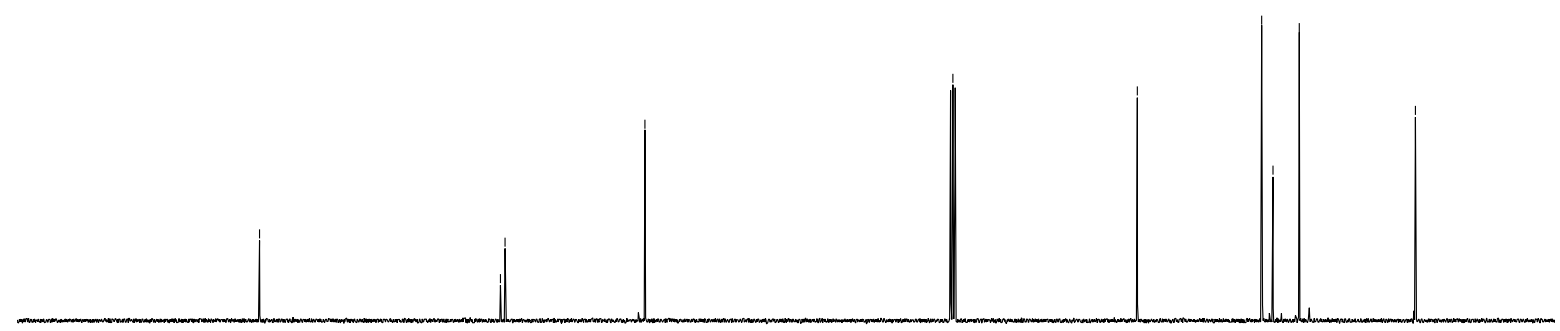

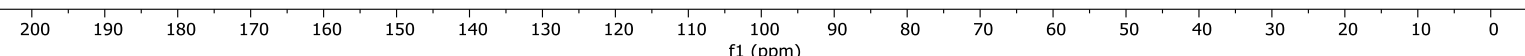


Methyl 3-((2,4-dibromo-1-methyl-1H-imidazol-5-yl)thio)propanoate (3f)<smiles>COC(=O)CCSc1c(Br)nc(Br)n1C</smiles>

$3 \mathbf{f}$ $1 \mathrm{H} \mathrm{NMR,} \mathrm{CDCl}_{3}, 400 \mathrm{MHz}$

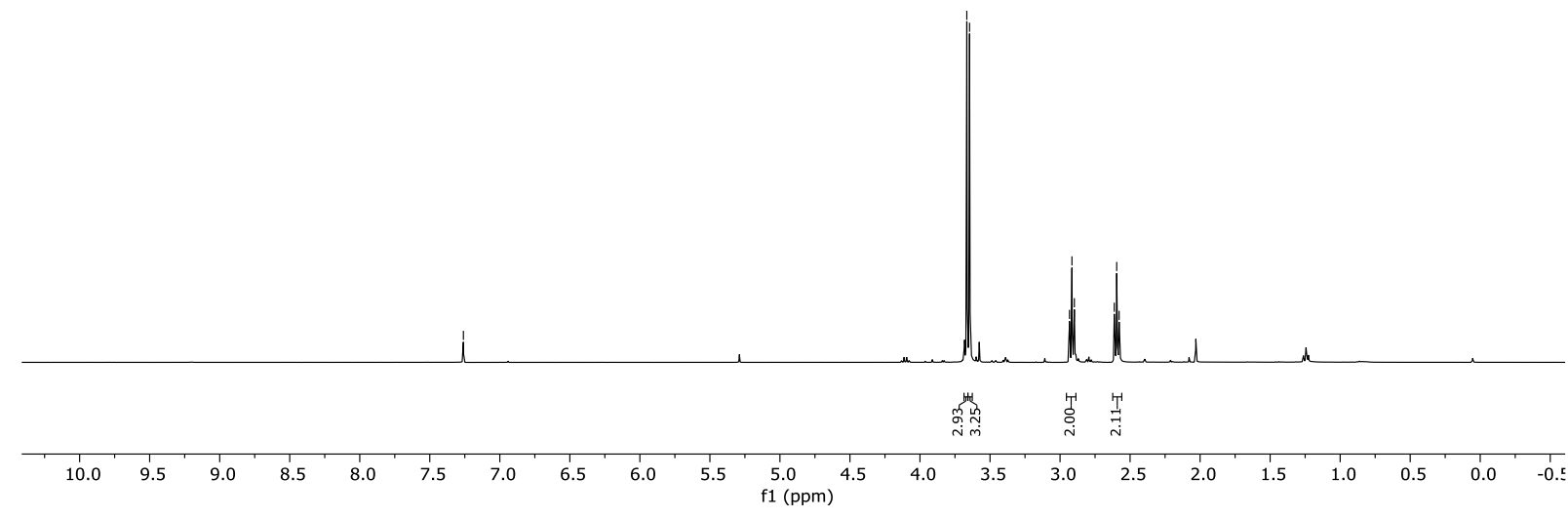<smiles>COC(=O)CCSc1c(Br)nc(Br)n1C</smiles>

$3 \mathbf{f}$ 13C NMR, $\mathrm{CDCl}_{3}, 101 \mathrm{MHz}$ 
2,4-Dibromo-5-((2,3-dichlorophenyl)thio)-1-methyl-1H-imidazole (3g)

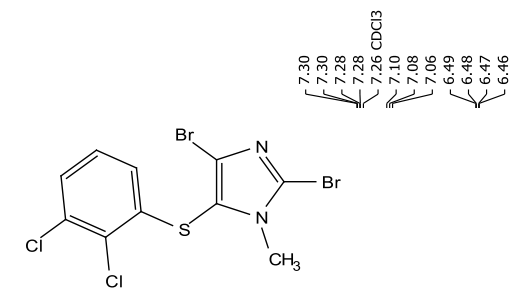

$3 \mathbf{g}$
$1 \mathrm{H} N M R$,

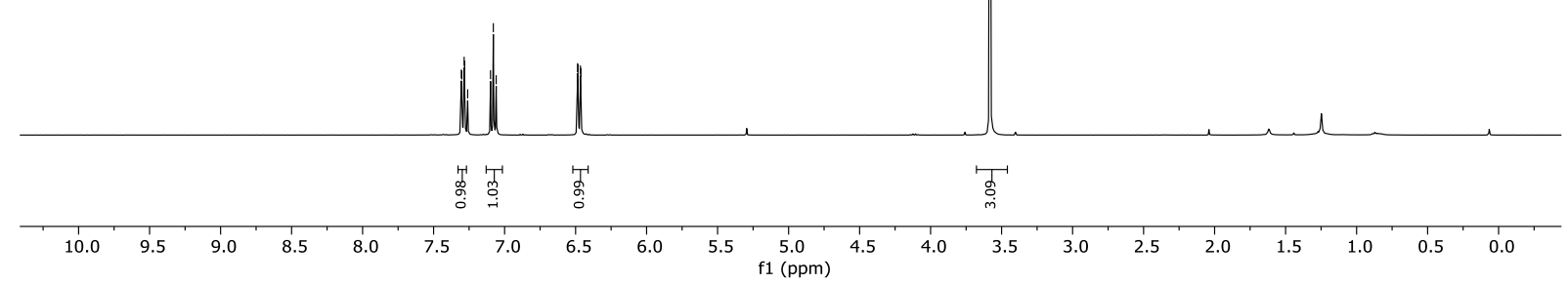
0
1
0<smiles>Cn1c(Br)nc(Br)c1Sc1cccc(Cl)c1Cl</smiles>

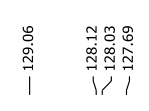

3g
$13 \mathrm{C}$ NMR, $\mathrm{CDCl}_{3}, 101 \mathrm{MHz}$

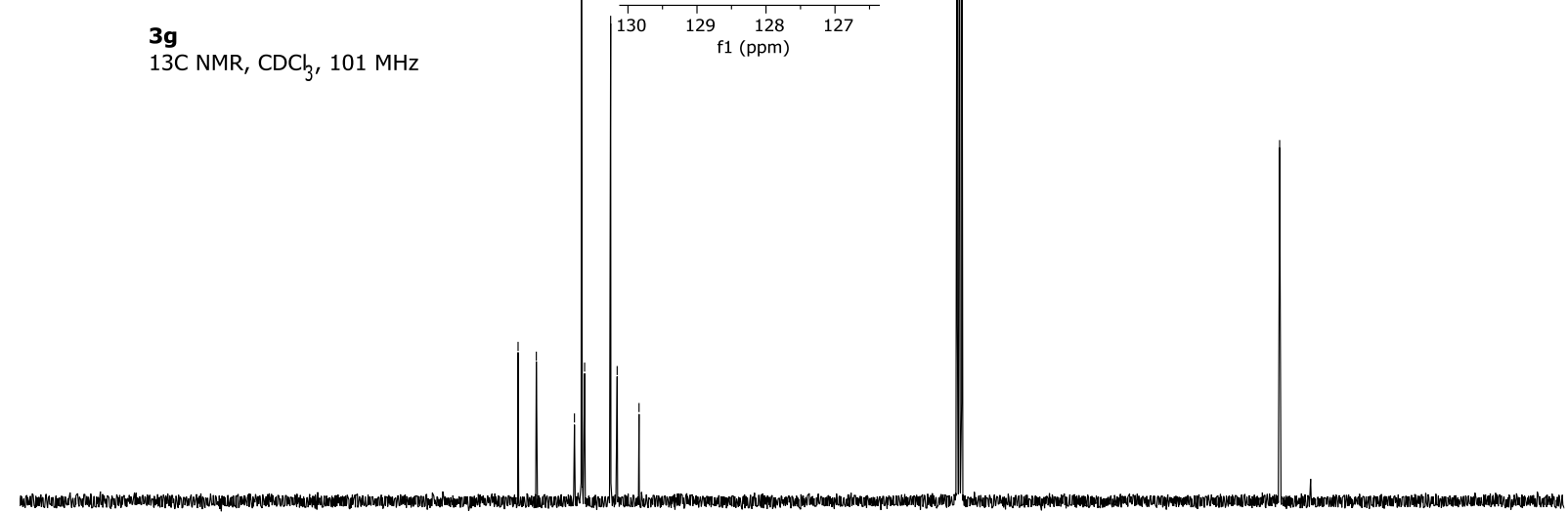

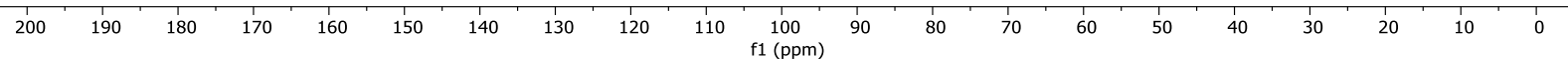


Methyl 3-((1-benzyl-2-bromo-4-chloro-1 $H$-imidazol-5-yl)thio)propanoate ( $\left.\mathbf{3} \mathbf{h}_{\text {major }}\right)$ and Methyl 3-((1-benzyl-4,5-dichloro-1H-imidazol-2-yl)thio)propanoate $\left(\mathbf{3} \mathbf{h}_{\text {minor }}\right)$

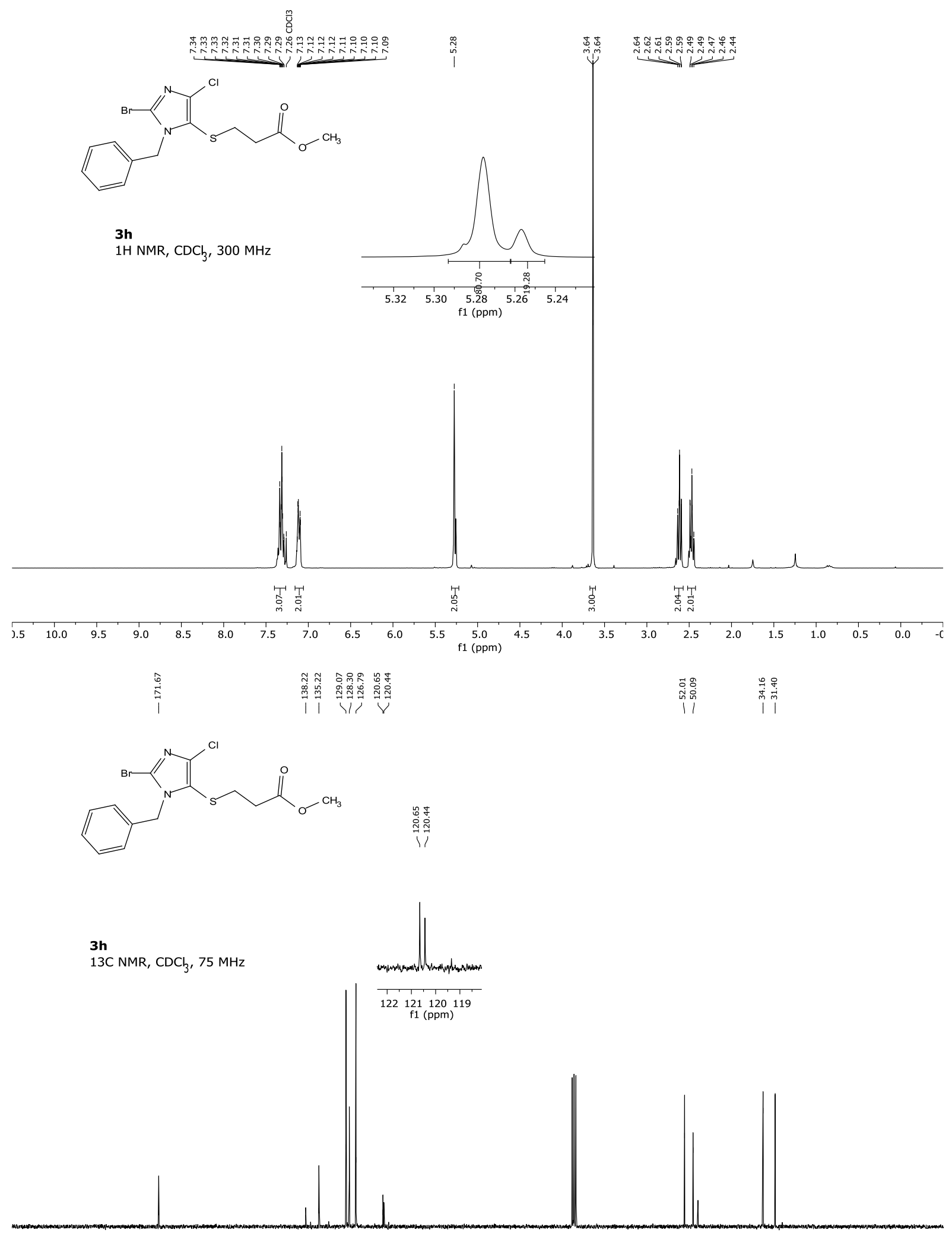


Methyl (S)-2-((tert-butoxycarbonyl)amino)-3-(5-((3-methoxy-3-oxopropyl)thio)-1-methyl$1 H$-imidazol-4-yl)propanoate (3i)
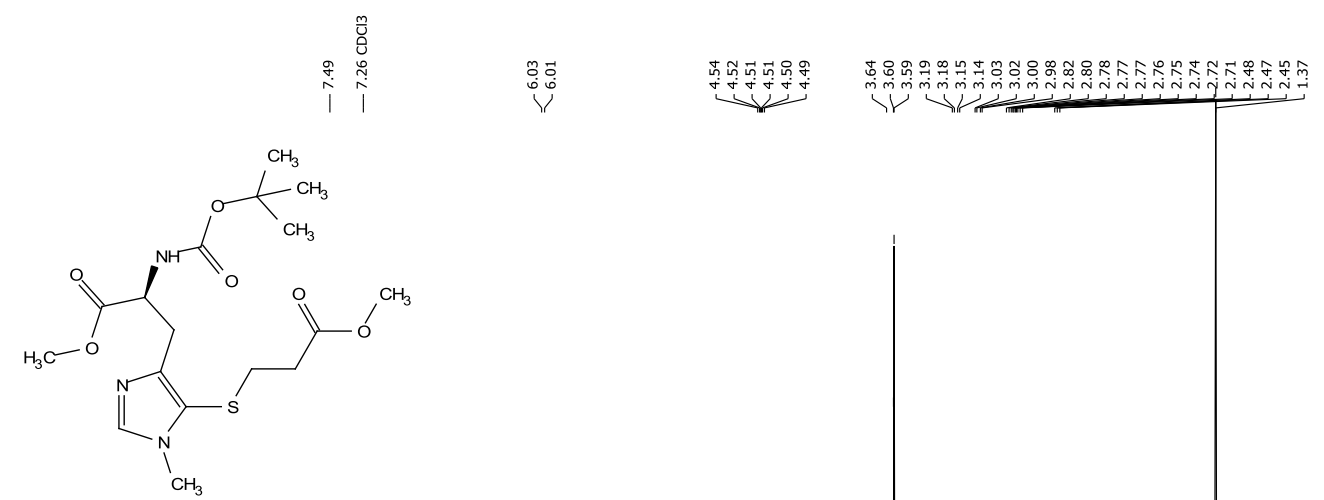

$1 \mathrm{H} N \mathrm{NR}, \mathrm{CDCl}_{3}, 400 \mathrm{MHz}$

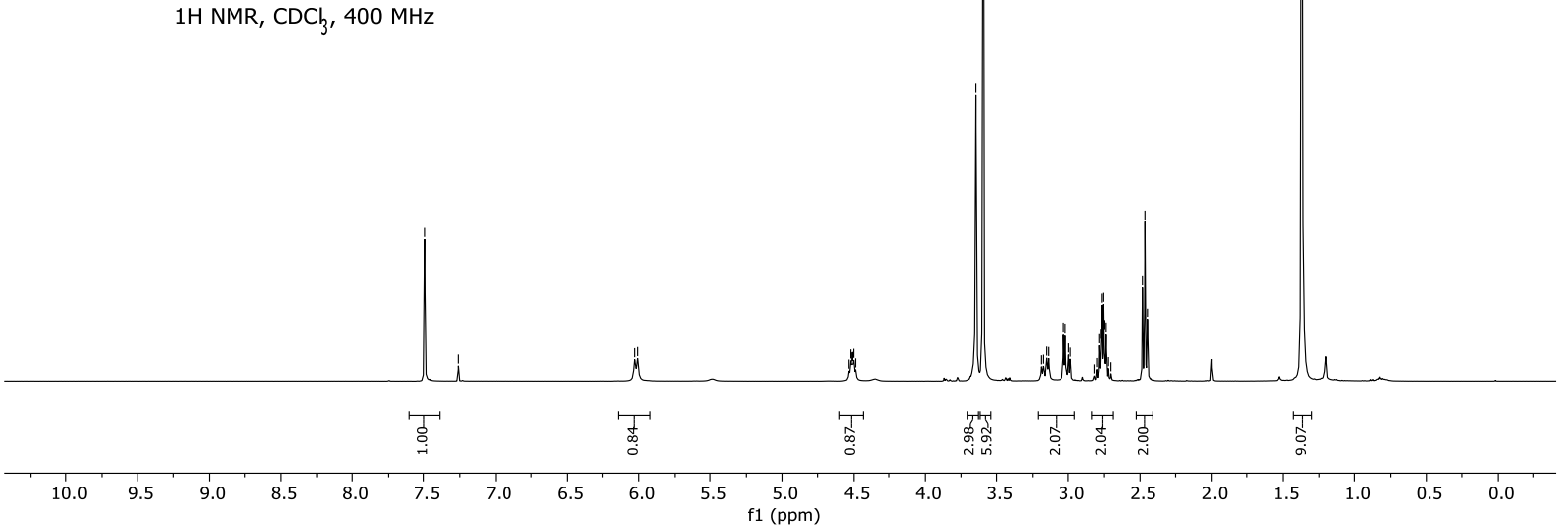

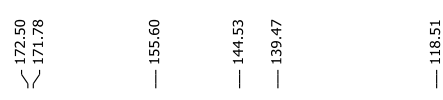
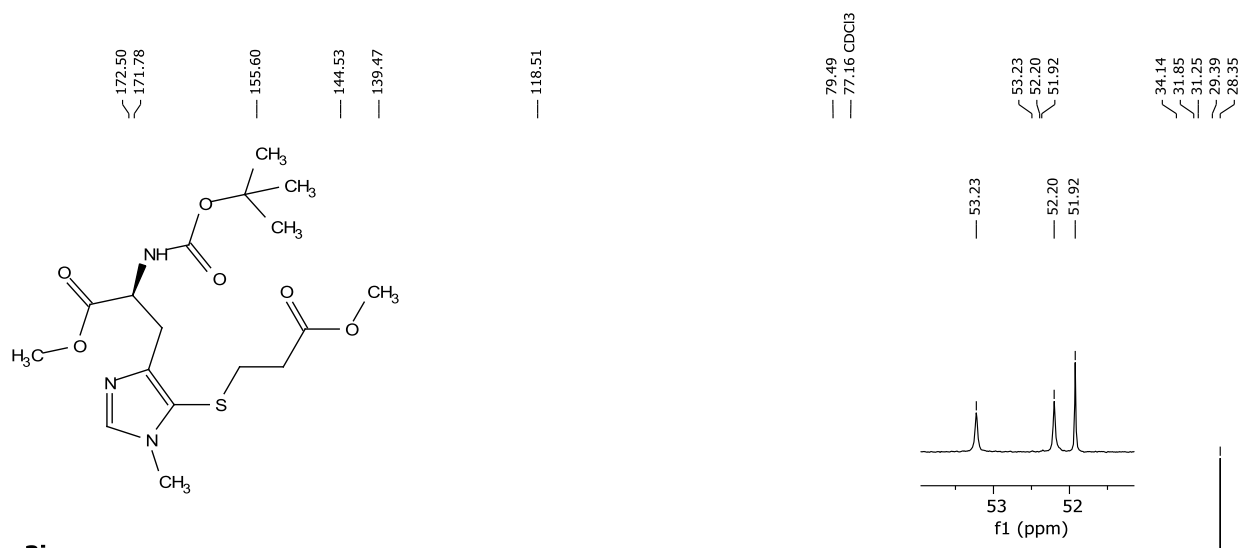

3i

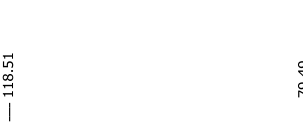

$13 \mathrm{C} \mathrm{NMR}, \mathrm{CDCl}_{3}, 101 \mathrm{MHz}$

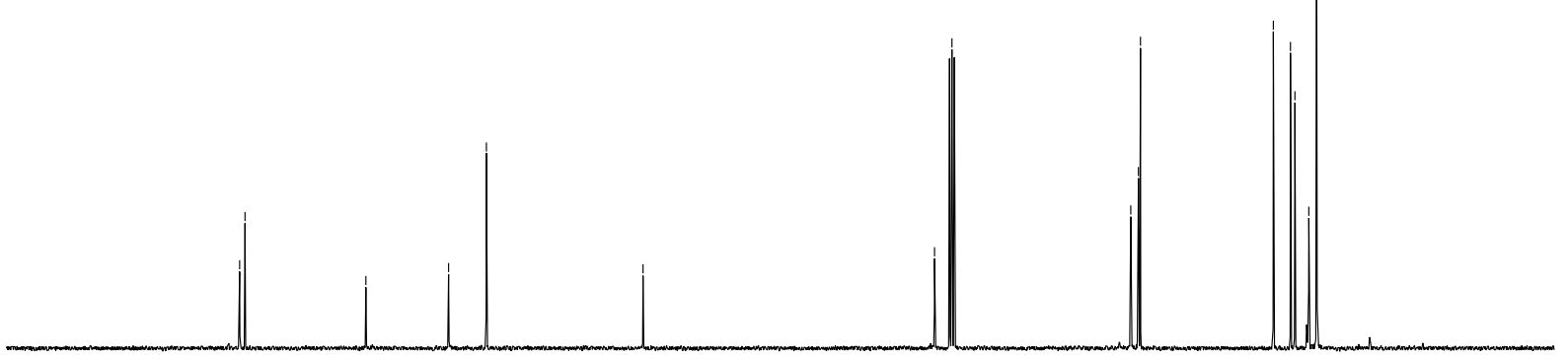

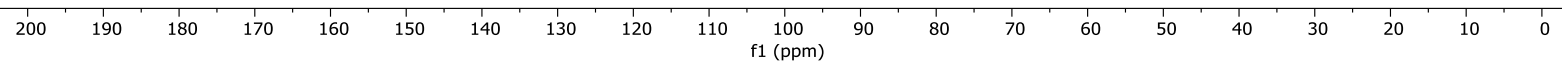


Methyl (S)-2-((tert-butoxycarbonyl)amino)-3-(2-chloro-5-((3-methoxy-3-oxopropyl)thio)-1methyl-1H-imidazol-4-yl)propanoate (3j)
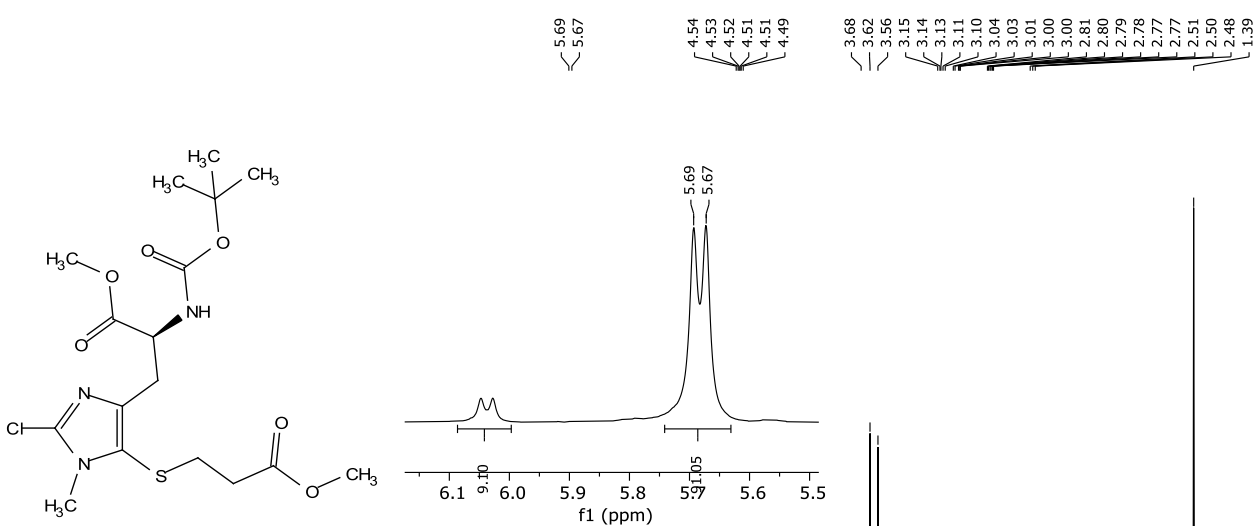

3j

$1 \mathrm{H} \mathrm{NMR}, \mathrm{CDCl}_{3}, 400 \mathrm{MHz}$

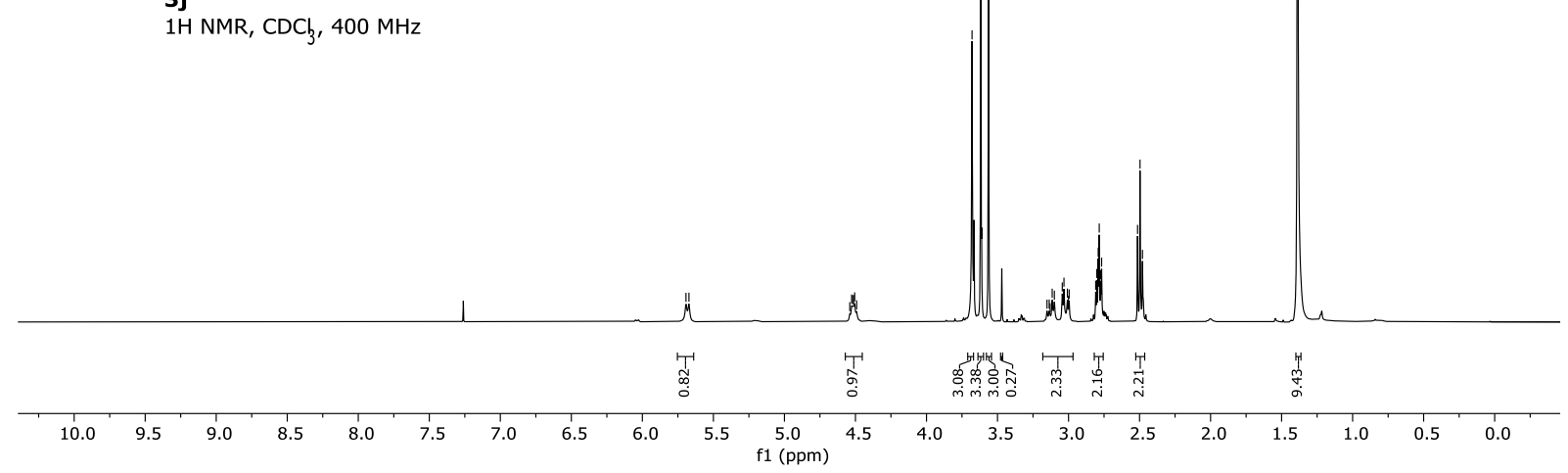

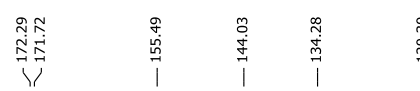

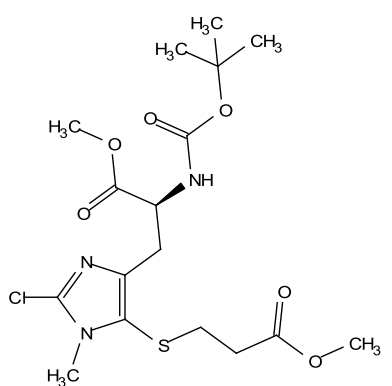

$3 \mathbf{j}$

$13 \mathrm{C} \mathrm{NMR}, \mathrm{CDCl}_{3}, 101 \mathrm{MHz}$

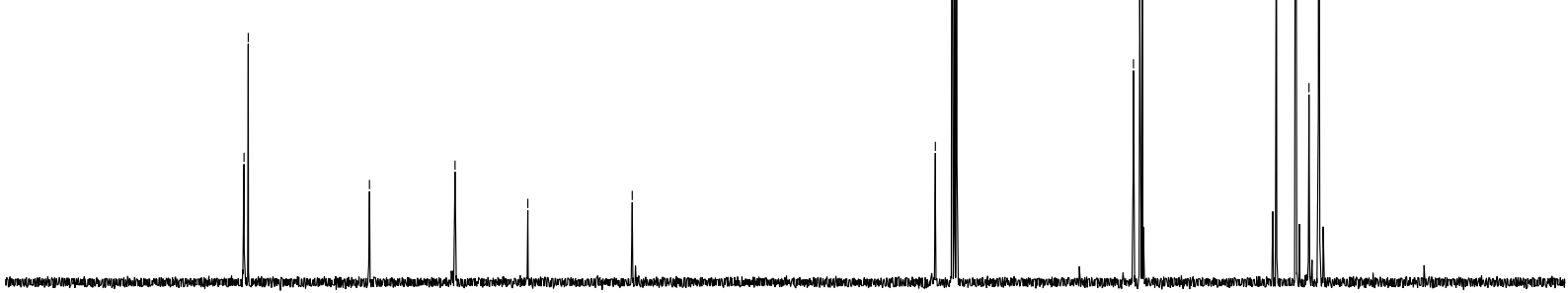


Methyl 3-((4-chloro-1-(2-chlorobenzyl)-1H-imidazol-5-yl)thio)propanoate (3k)

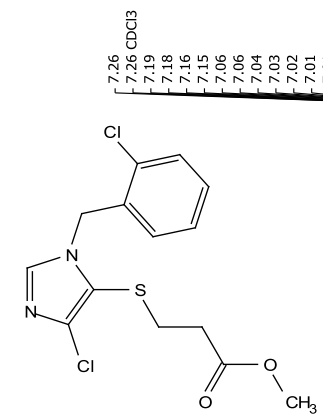

3k

$1 \mathrm{H} \mathrm{NMR}, \mathrm{CDCl}_{3}, 300 \mathrm{MHz}$
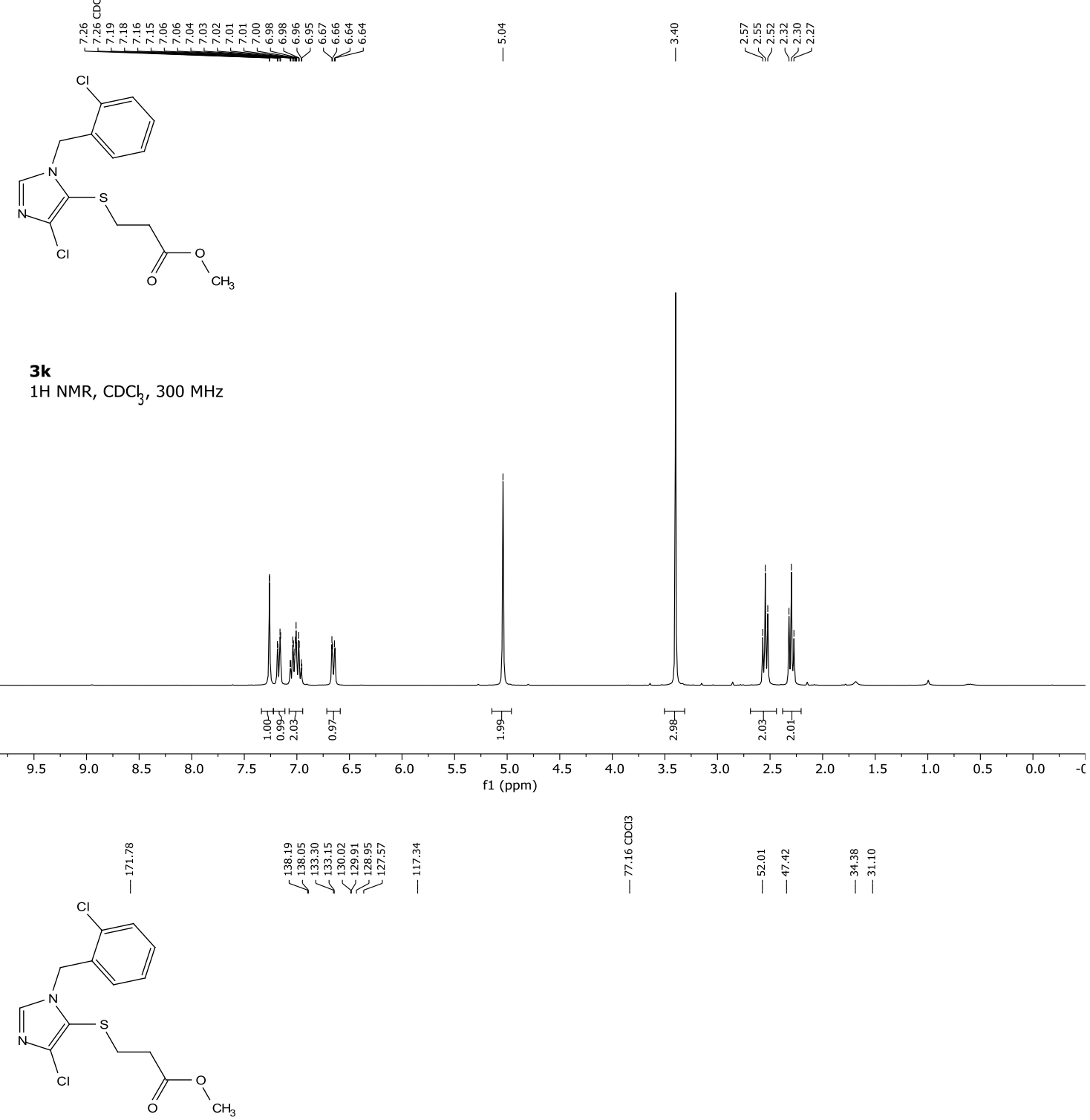

3k

$13 \mathrm{C} \mathrm{NMR,} \mathrm{CDCl}_{3}, 75 \mathrm{MHz}$

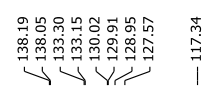

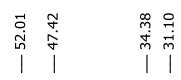

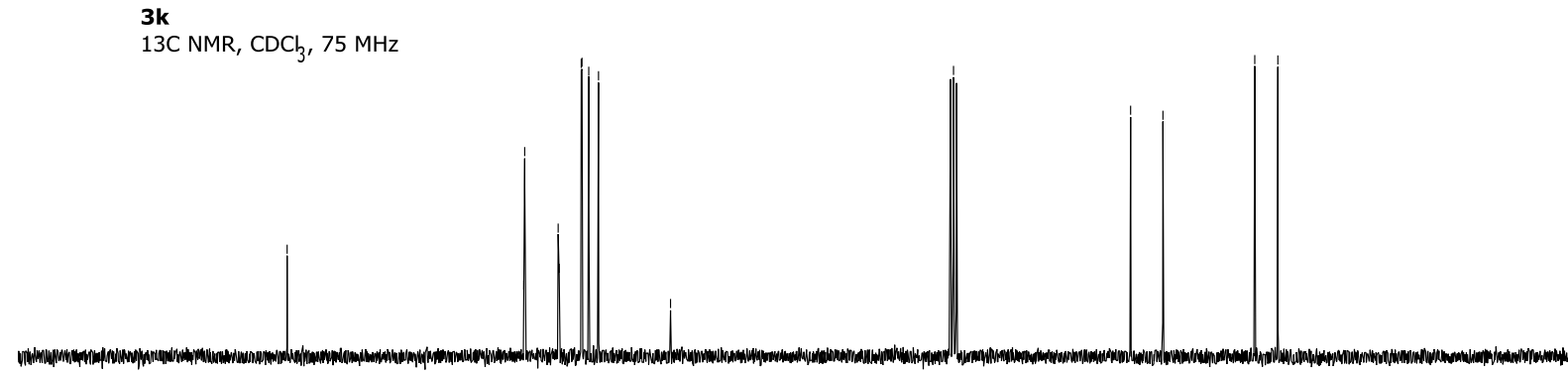

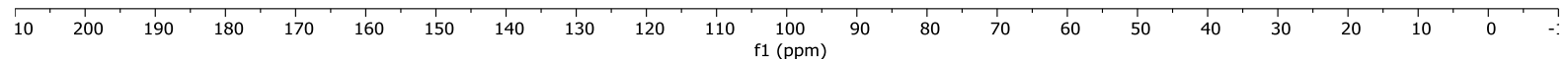


Methyl 3-((1-(4-bromobenzyl)-4-chloro-1H-imidazol-5-yl)thio)propanoate (3I)
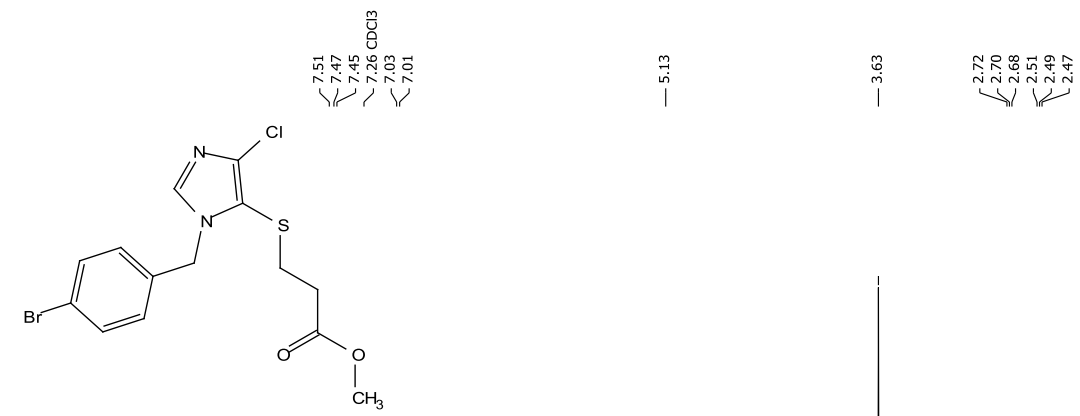

3I

$1 \mathrm{H} \mathrm{NMR}, \mathrm{CDCl}_{3}, 400 \mathrm{MHz}$

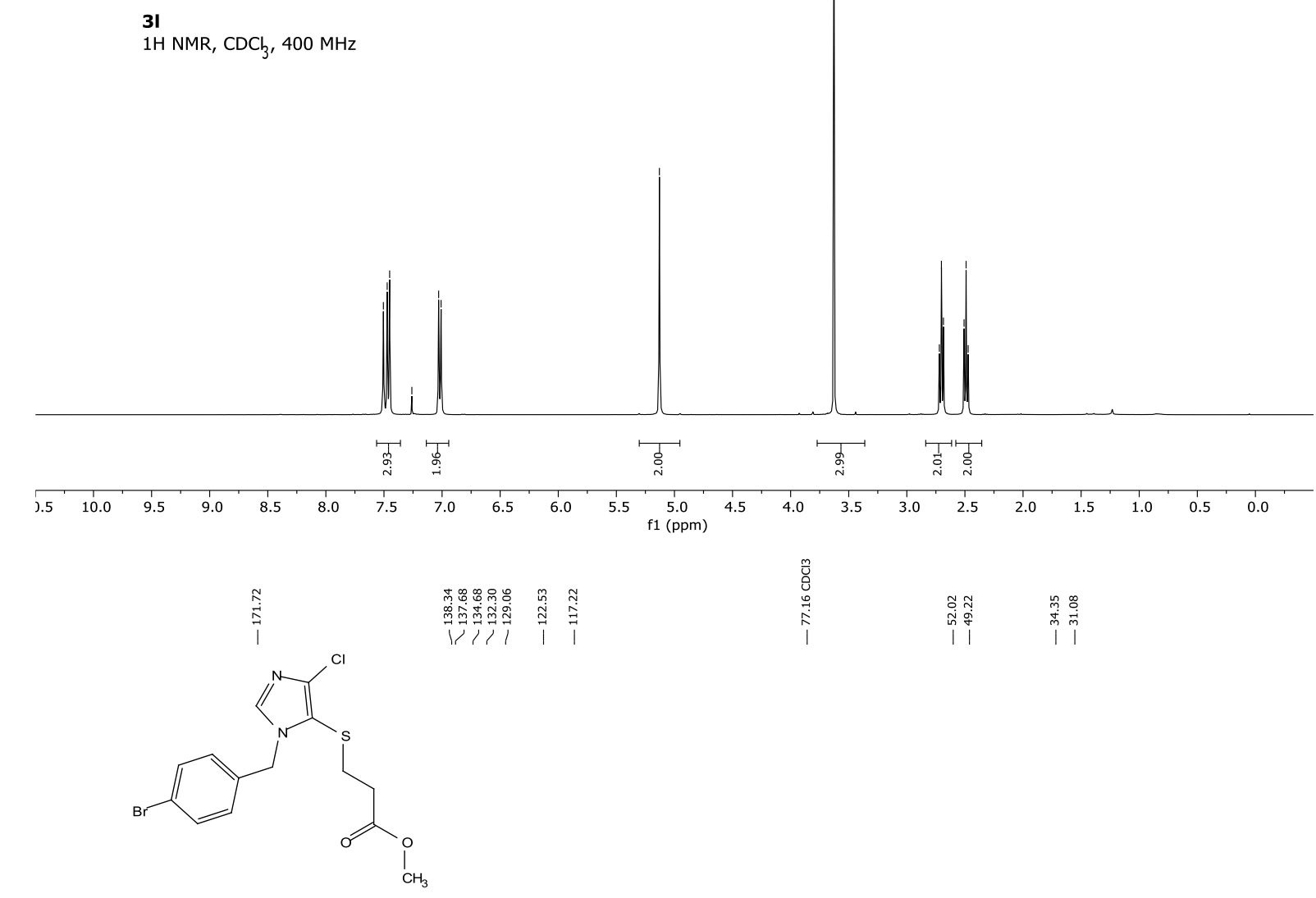

3I

$13 \mathrm{C} \mathrm{NMR,} \mathrm{CDCl}_{3}, 101 \mathrm{MHz}$

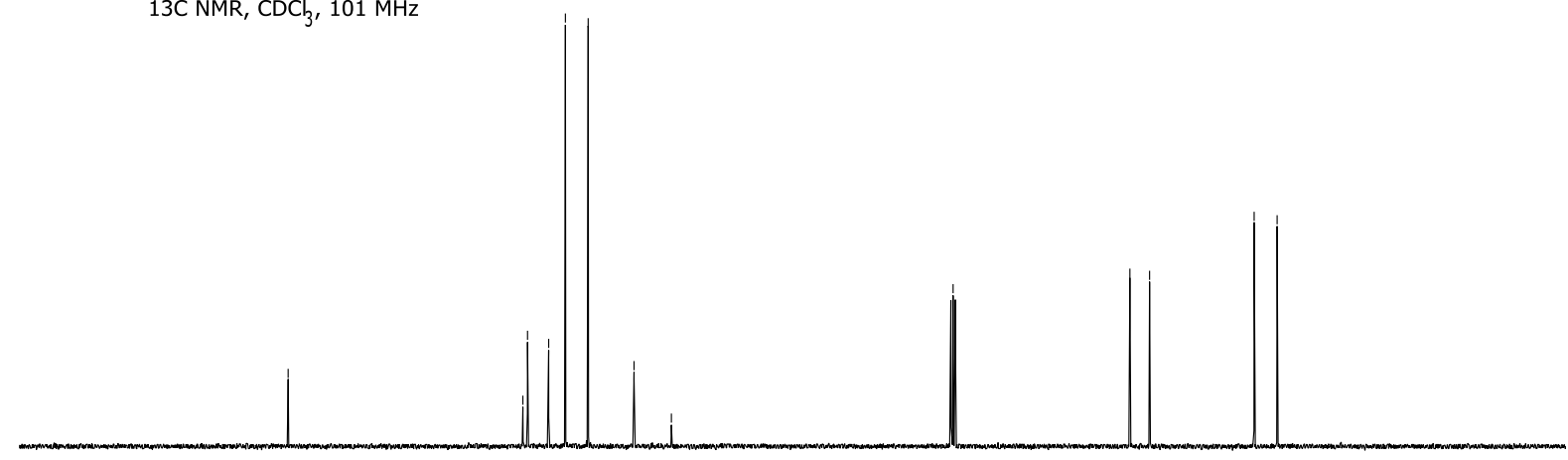

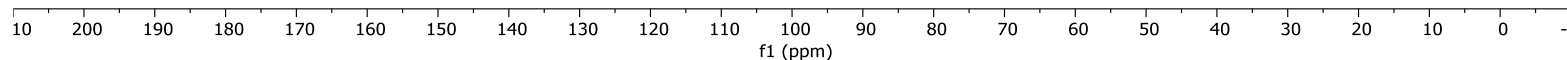


Methyl 3-((4-chloro-1-(2-(6-chloronicotinamido)ethyl)-1H-imidazol-5-yl)thio)propanoate $(\mathbf{3 m})$
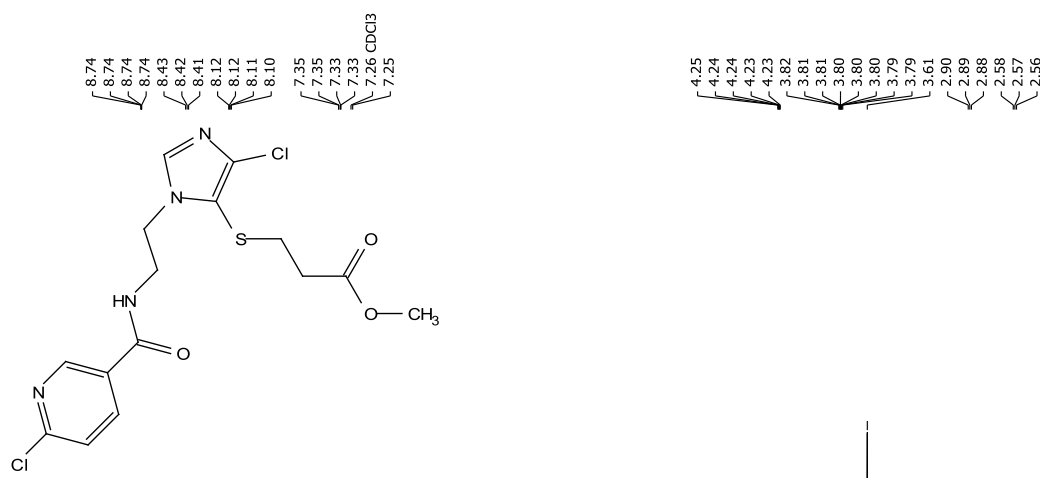

$1 \mathrm{H}$ NMR, $\mathrm{CDCl}_{3}, 600 \mathrm{MHz}$

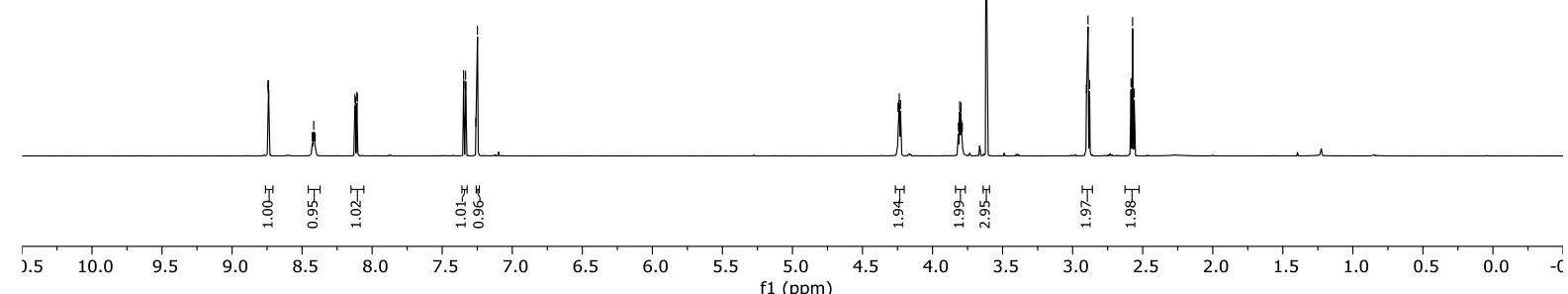

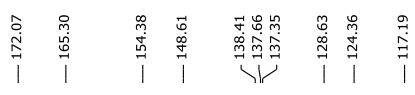

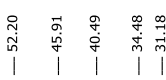

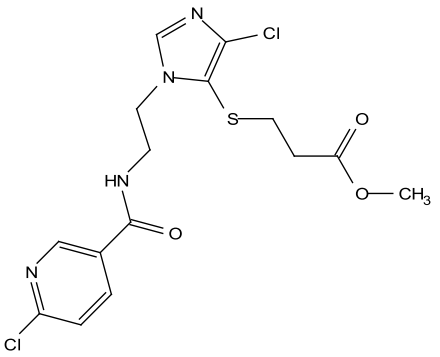

$13 \mathrm{C}$ NMR, $\mathrm{CDCl}_{3}, 151 \mathrm{MHz}$

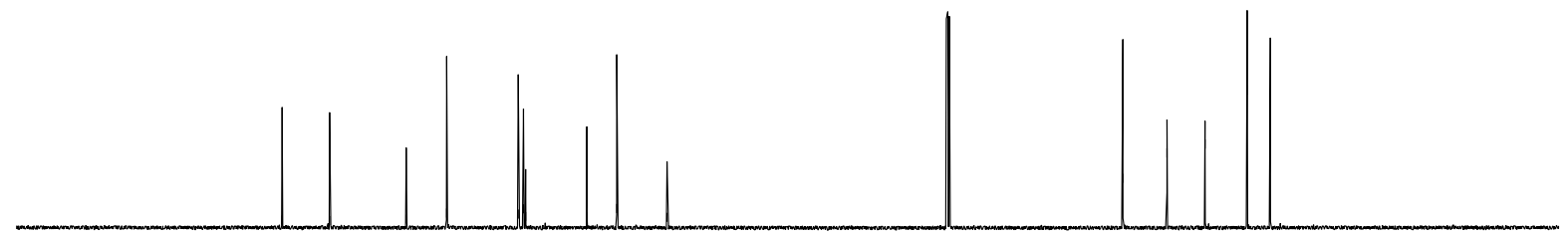

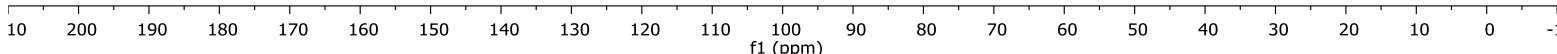


Methyl 3-((3-bromothiophen-2-yl)thio)propanoate (3n)

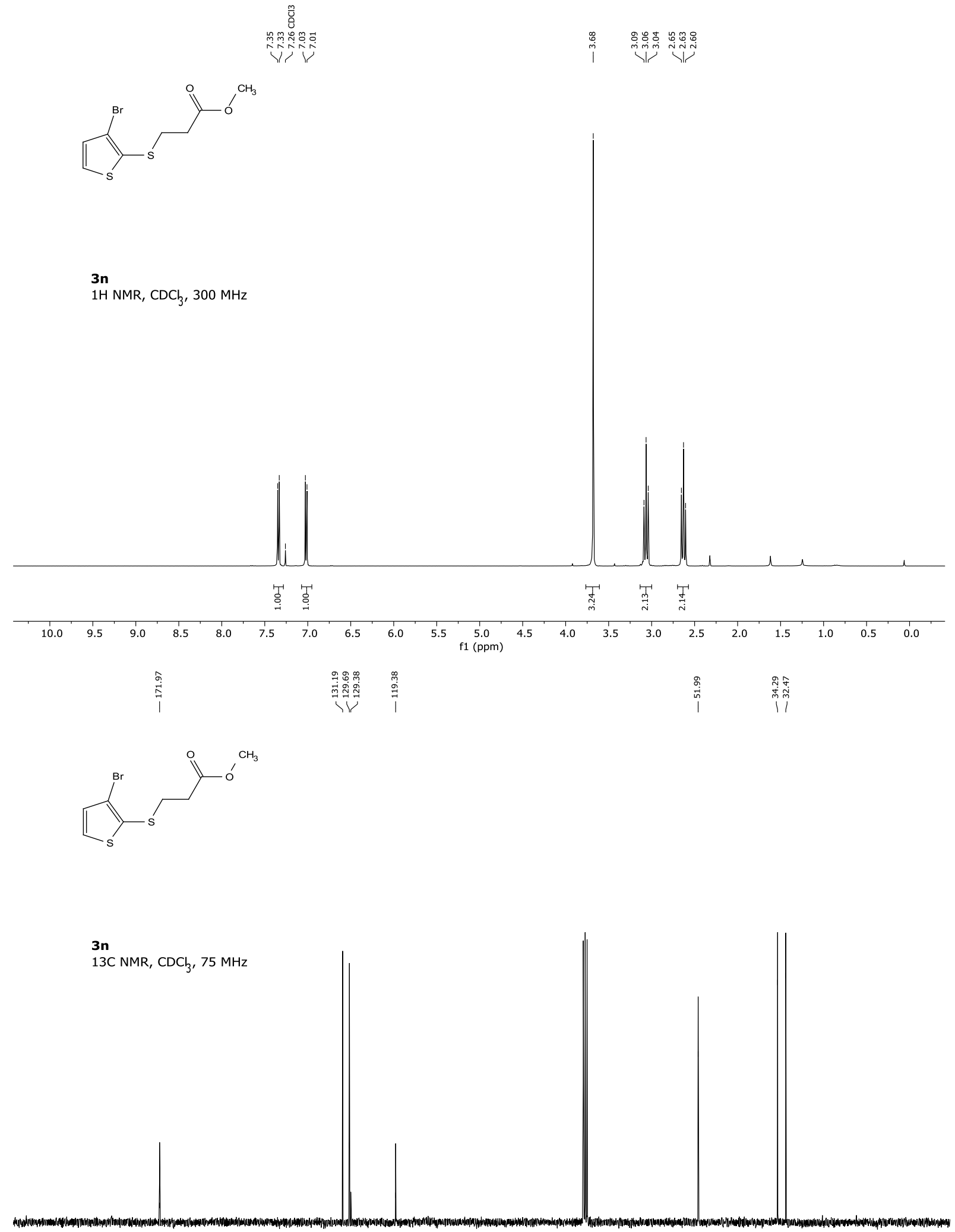

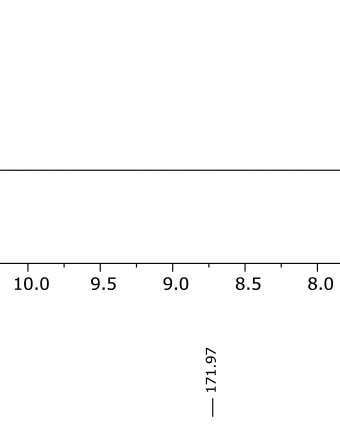

200

$\begin{array}{llllll}180 & 170 & 160 & 150 & 140\end{array}$

$10 \quad 100$

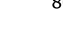

(2)


Methyl 3-((5-bromothiophen-2-yl)thio)propanoate (3o)

Impurity: 3-(Thiophen-2-ylthio)propanoate (10 mol\%)

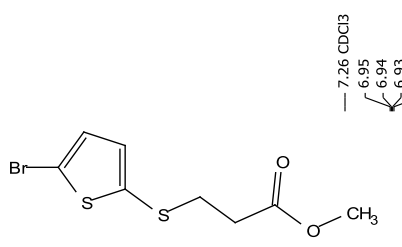

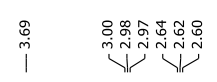

3o
$1 \mathrm{H} N \mathrm{NR}, \mathrm{CDCl}_{3}, 400 \mathrm{MHz}$
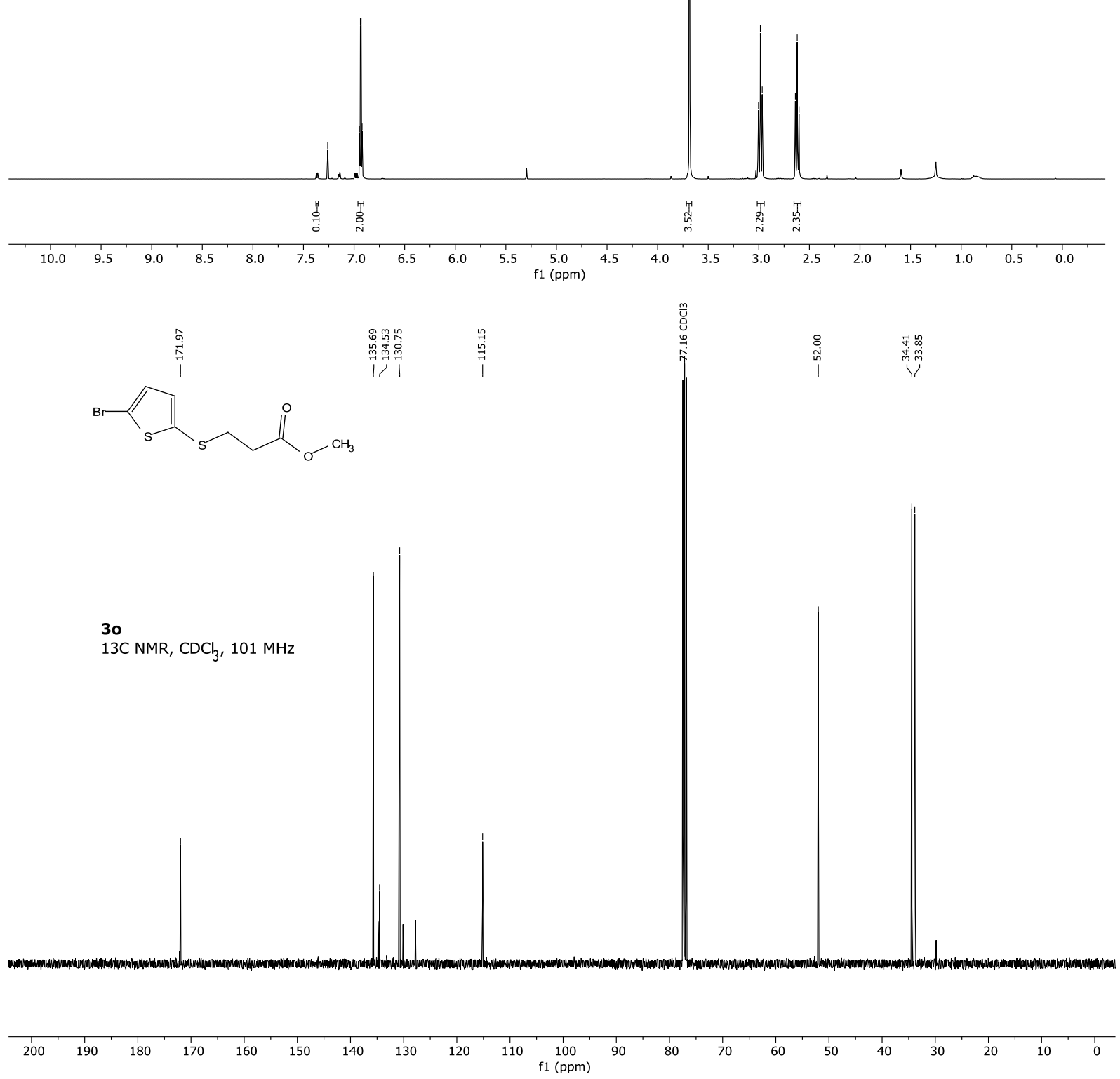

S107 
Methyl 3-((5-phenylfuran-2-yl)thio)propanoate (3p)

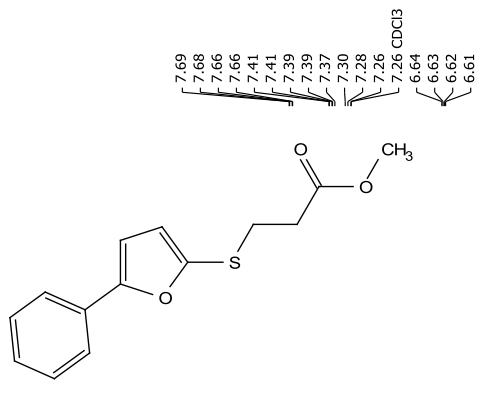

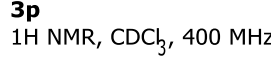

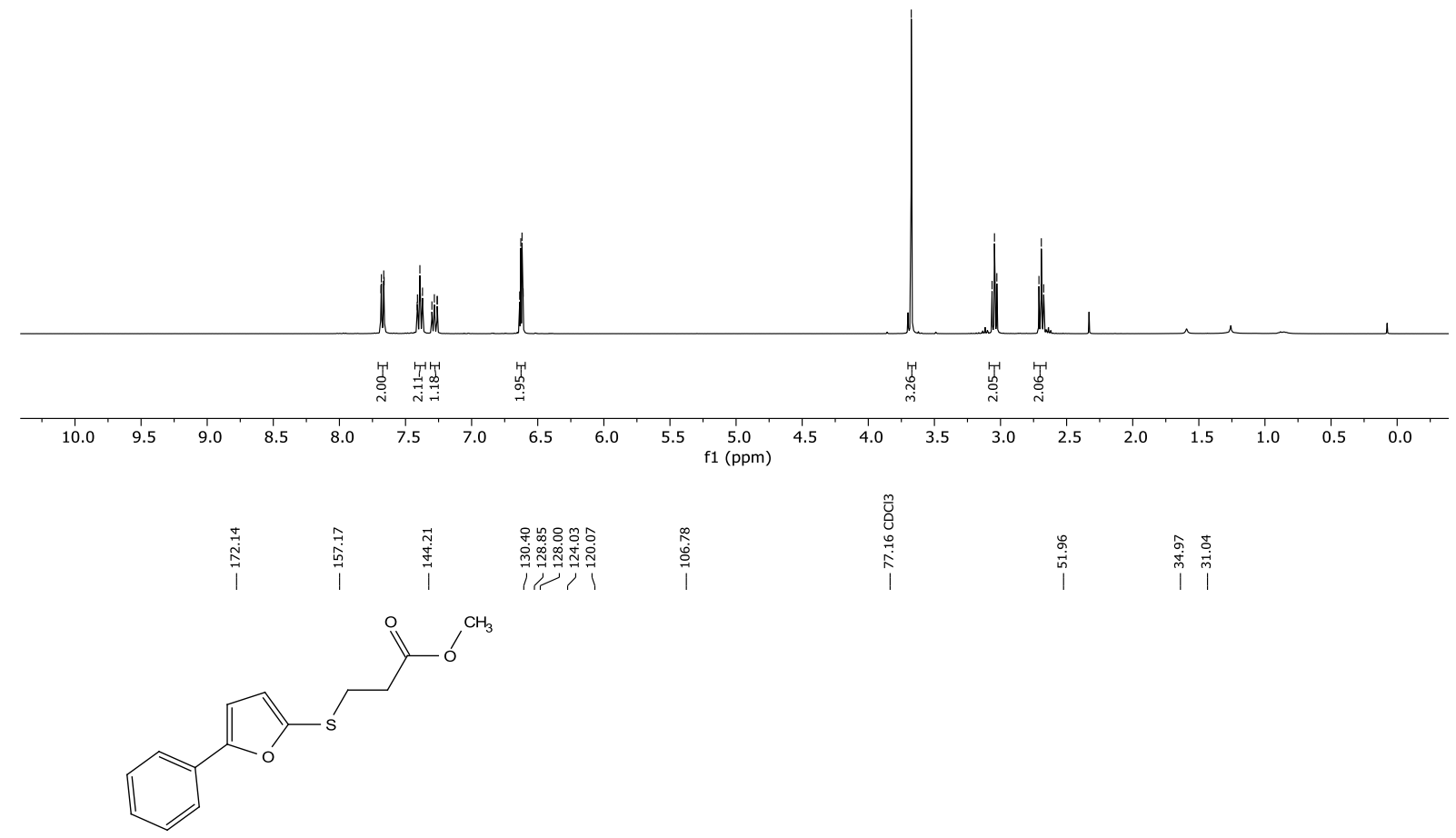

3p $13 \mathrm{CNMR}, \mathrm{CDCl}_{3}, 101$

$\mathrm{MHz}$

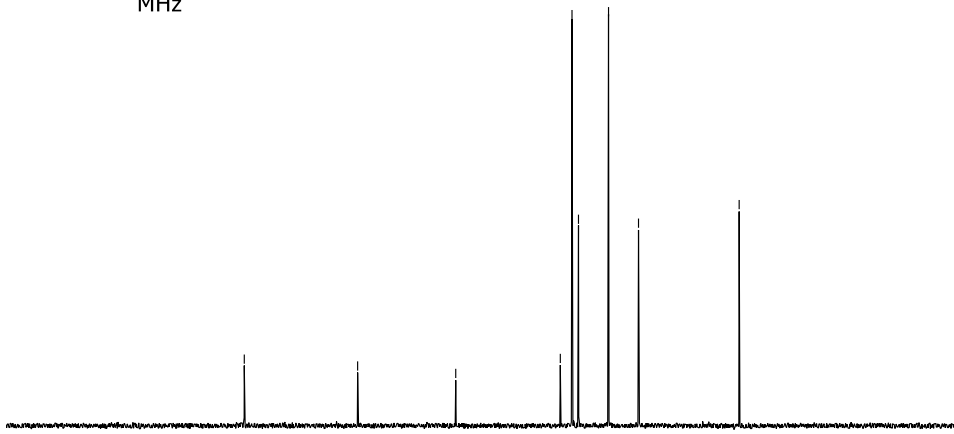

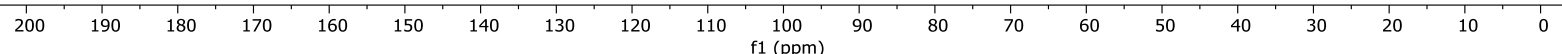


Methyl 3-((4-bromo-5-phenylfuran-2-yl)thio)propanoate (3q)

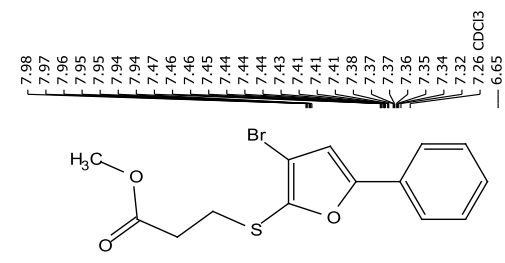

3q

$1 \mathrm{H}$ NMR, $\mathrm{CDCl}_{3}, 400 \mathrm{MHz}$
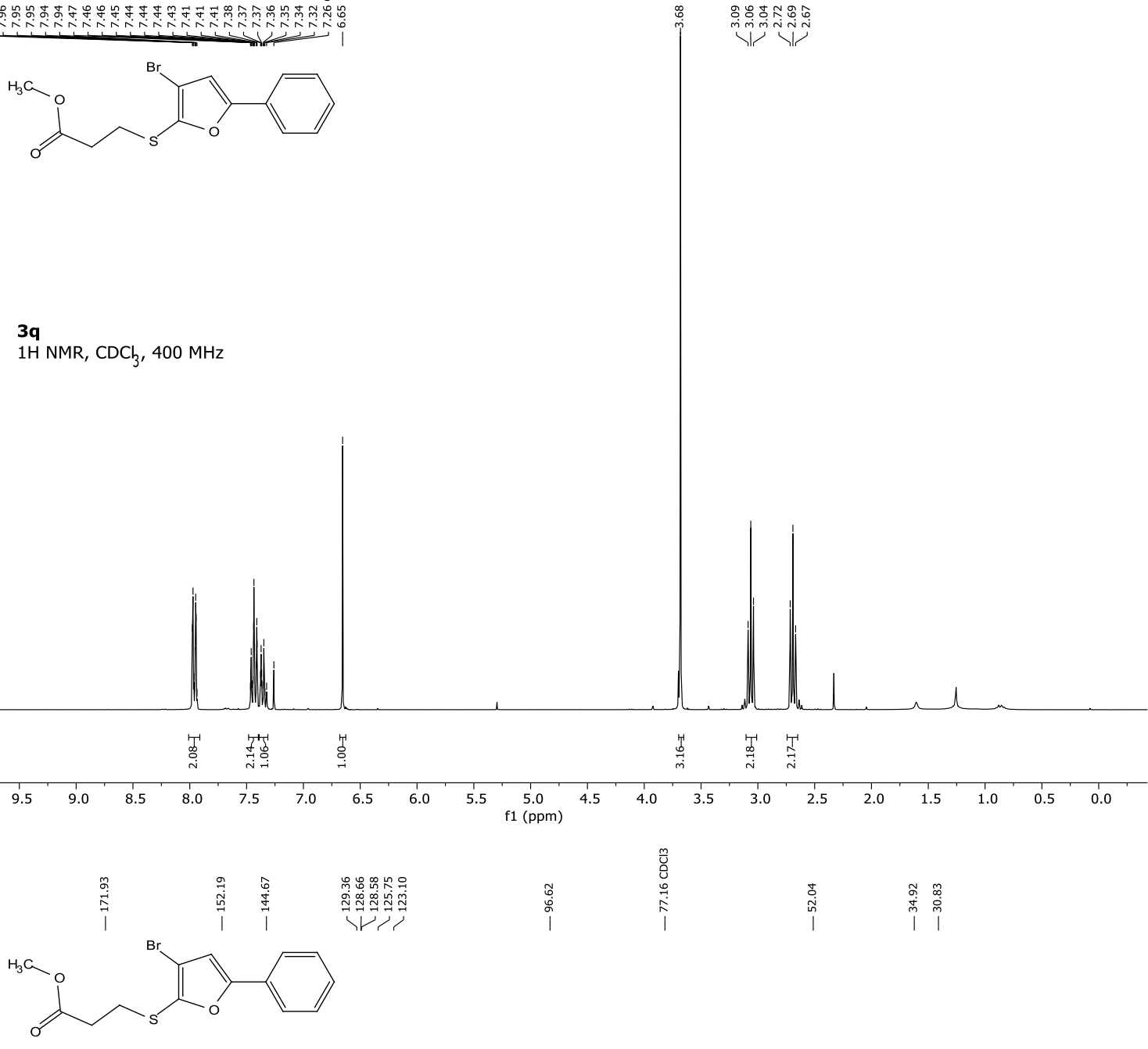

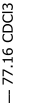

|

3q
13C NMR, $\mathrm{CDCl}_{3}, 101 \mathrm{MHz}$
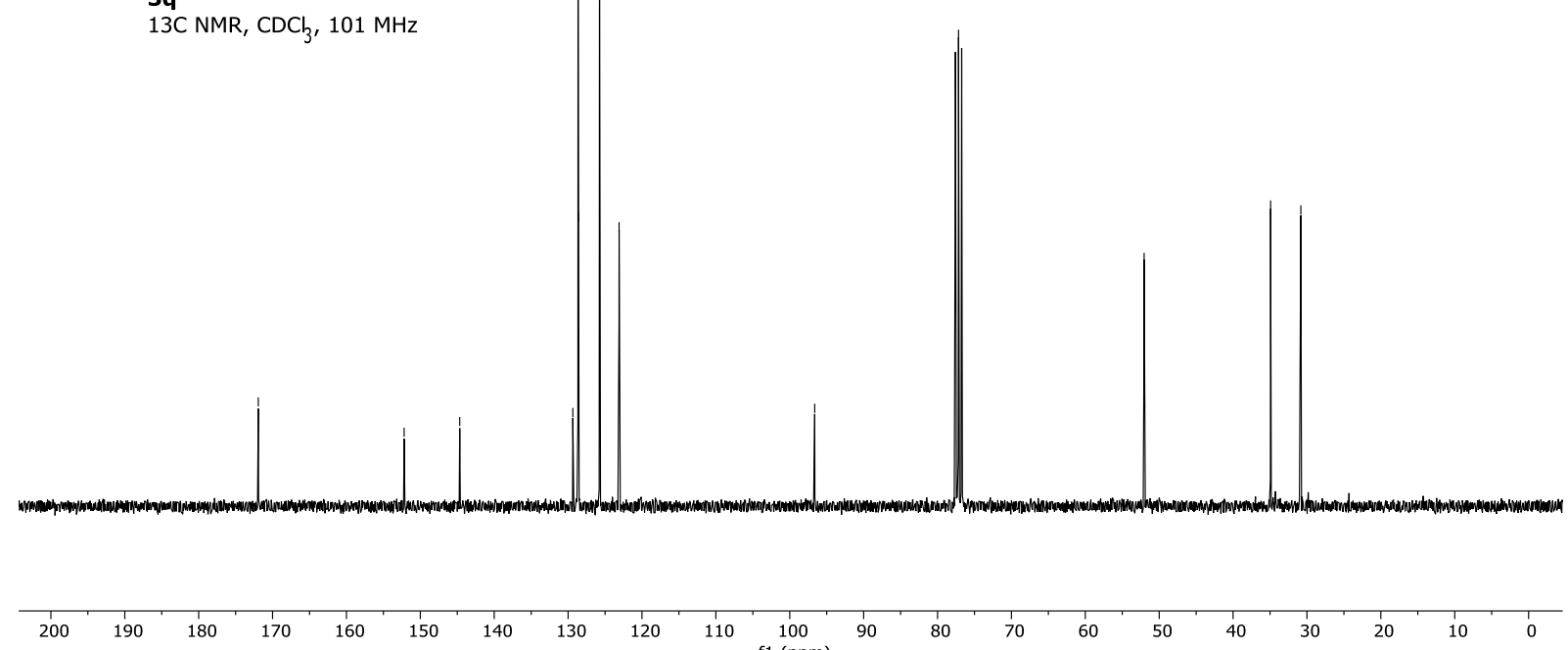

$10 \begin{gathered}100 \\ \mathrm{f} 1(\mathrm{ppm})\end{gathered}$

$90 \quad 80$

$\begin{array}{llll}70 & 60 & 1 & 10\end{array}$

$30 \quad 20 \quad 10 \quad 0$ 
Methyl 3-((1-methyl-5-phenyl-1H-pyrrol-2-yl)thio)propanoate (3r)

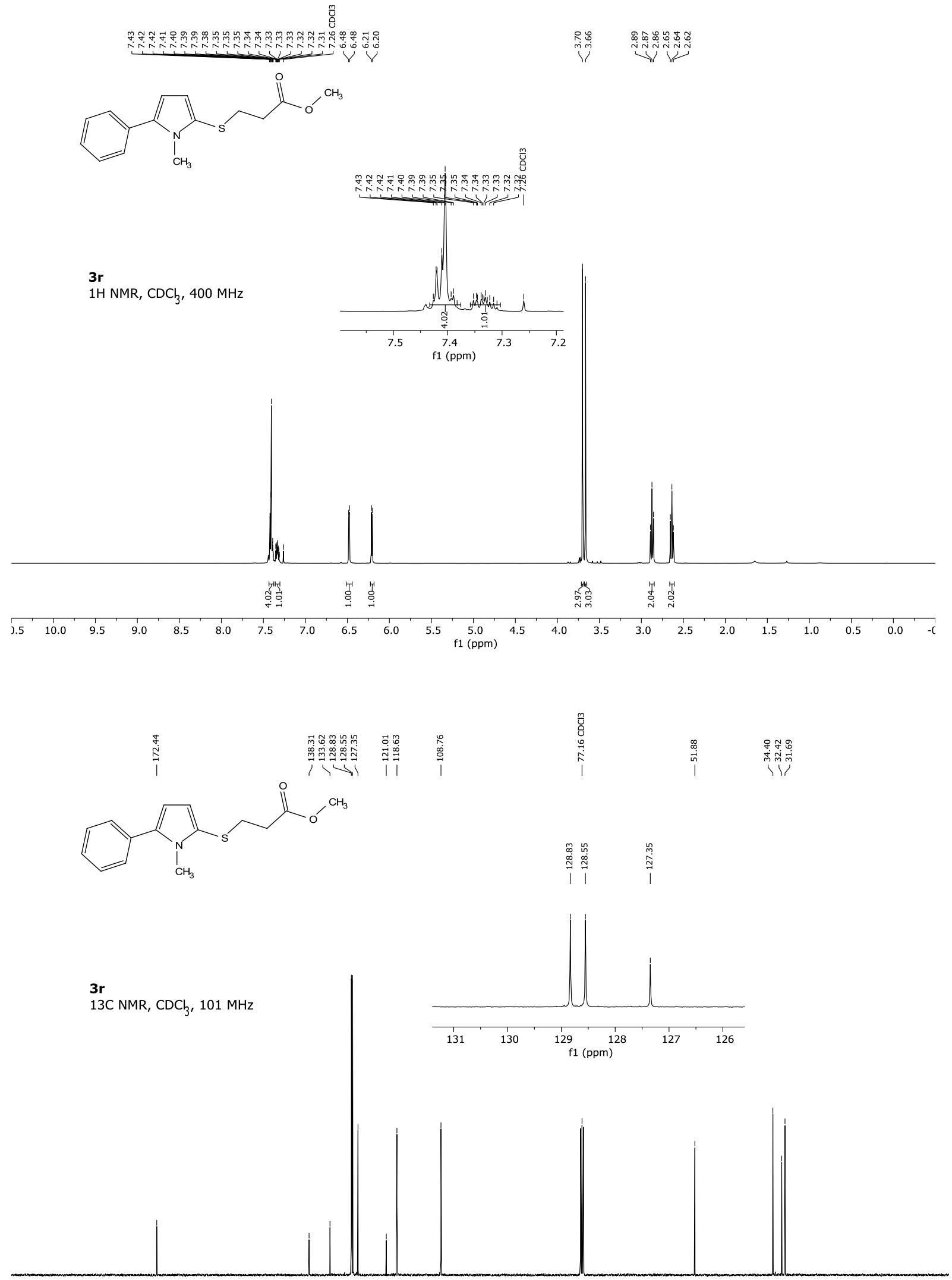

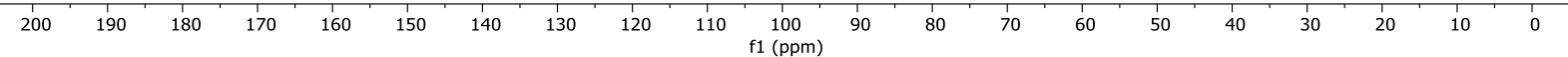


Methyl 3-((3,4-dichloro-1-methyl-5-phenyl-1H-pyrrol-2-yl)thio)propanoate (3s)

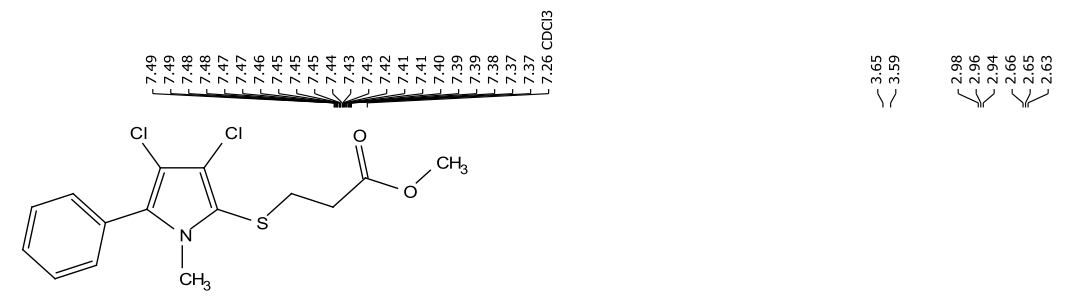

3s

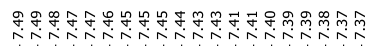

$1 \mathrm{H} \mathrm{NMR}, \mathrm{CDCl}_{3}, 400 \mathrm{MHz}$
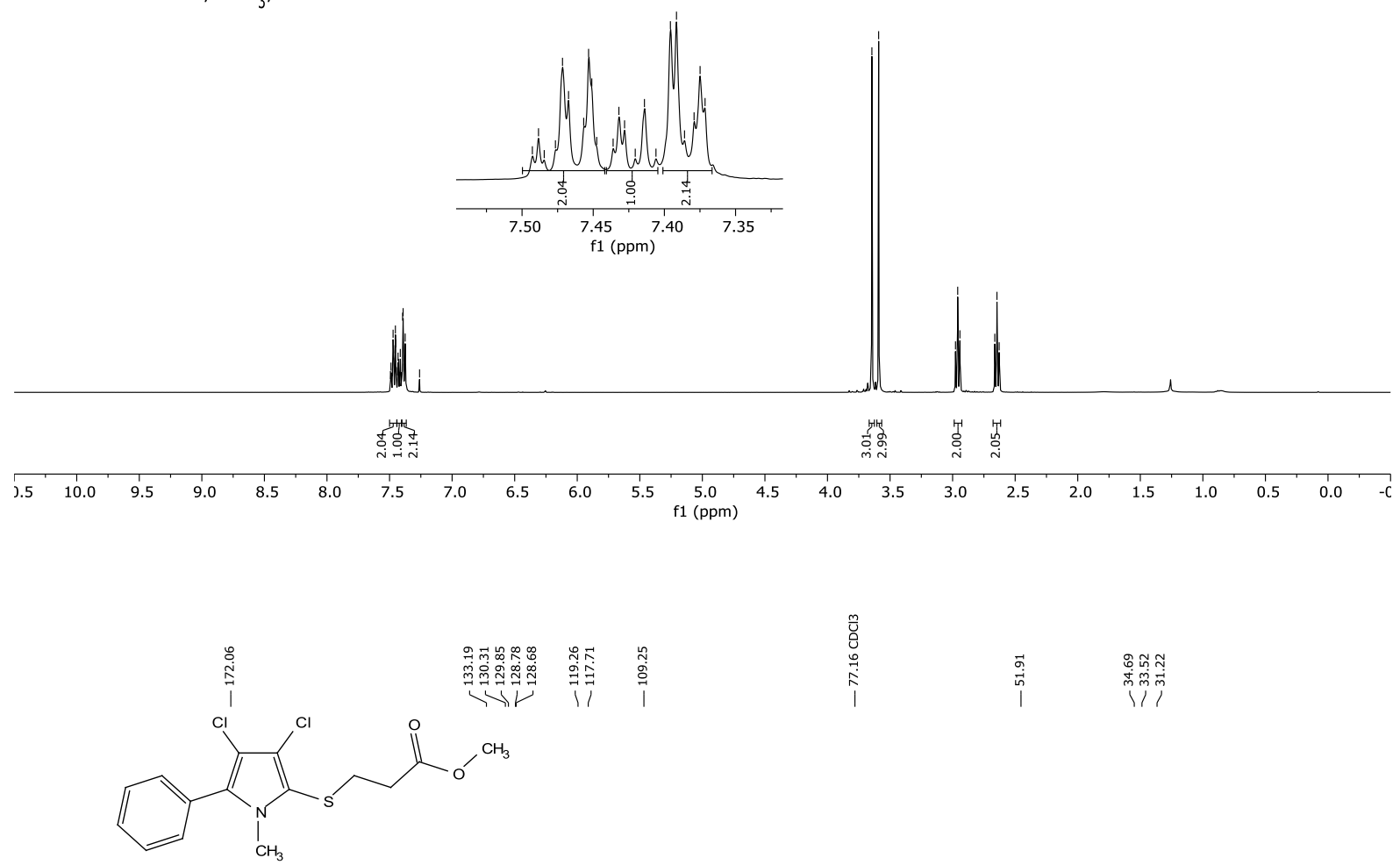

$3 s$

13C NMR, $\mathrm{CDCl}_{3}, 101 \mathrm{MHz}$

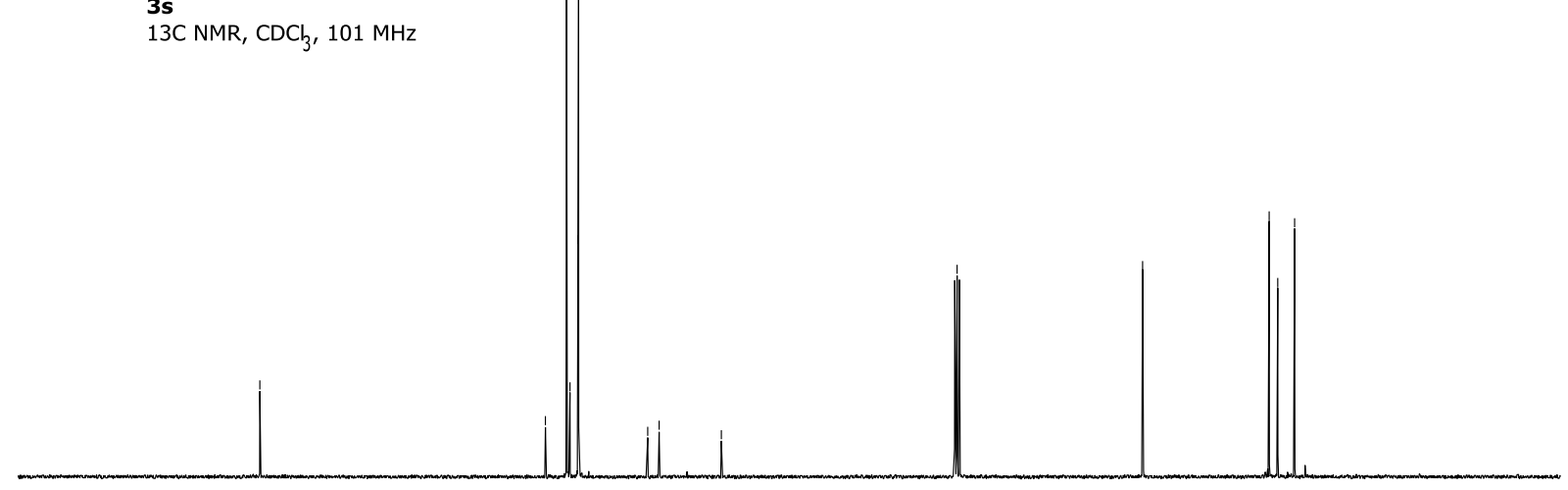

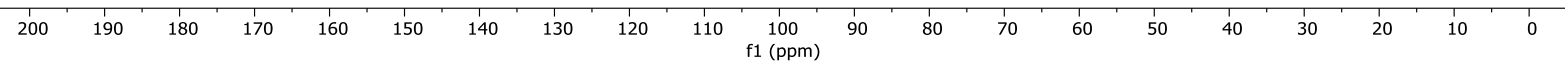


Methyl 3-((3,4-dibromo-1-methyl-5-phenyl-1H-pyrrol-2-yl)thio)propanoate (3t)
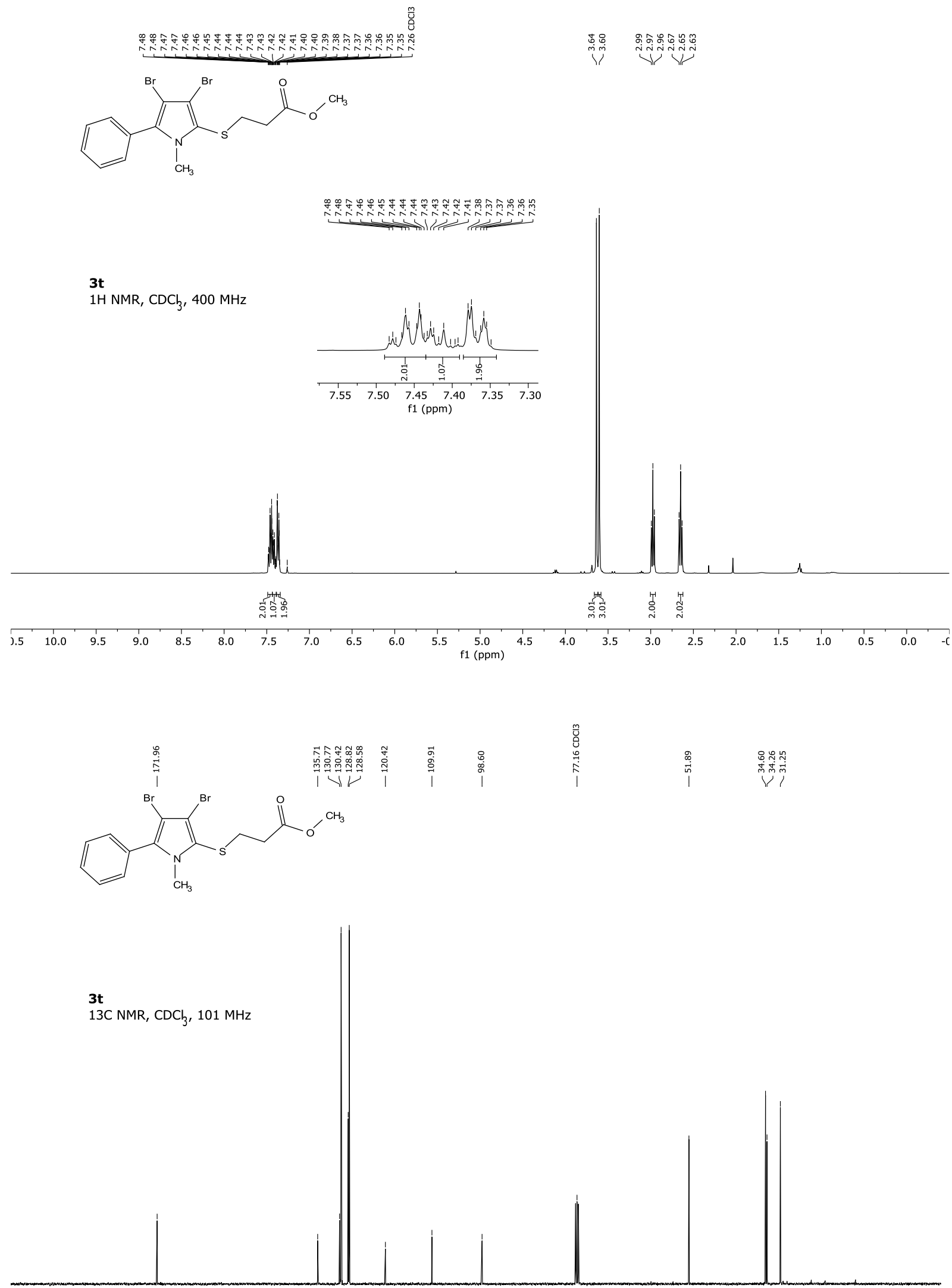

(c)

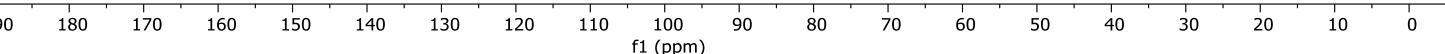


Dimethyl 3,3'-((1-methyl-1H-pyrrole-2,5-diyl)bis(sulfanediyl))dipropionate (3u)

Impurity: Dimethyl 3,3'-disulfanediyldipropionate (10 mol\%)<smiles>COC(=O)CCSc1ccc(SCCC(=O)OC)n1C</smiles>

$1 \mathrm{H}$ NMR, CDCb, $400 \mathrm{MHz}$
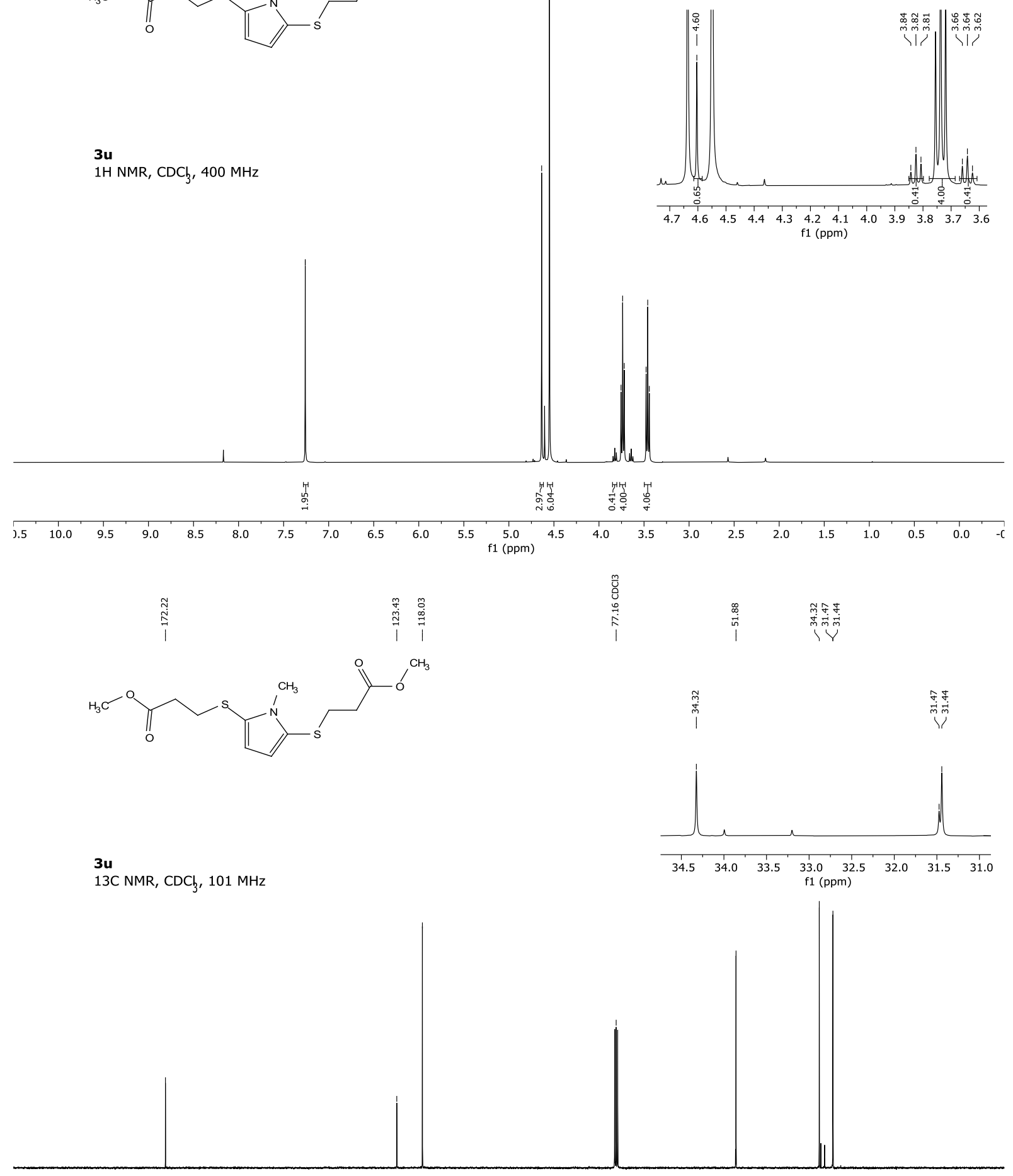
Methyl 3-((2-aminothiazol-5-yl)thio)propanoate (3v)
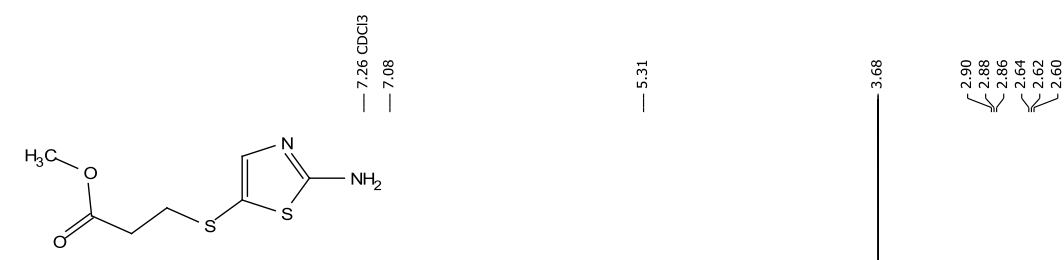

$1 \mathrm{H} \mathrm{NMR}, \mathrm{CDCl}_{3}, 400 \mathrm{MHz}$

i
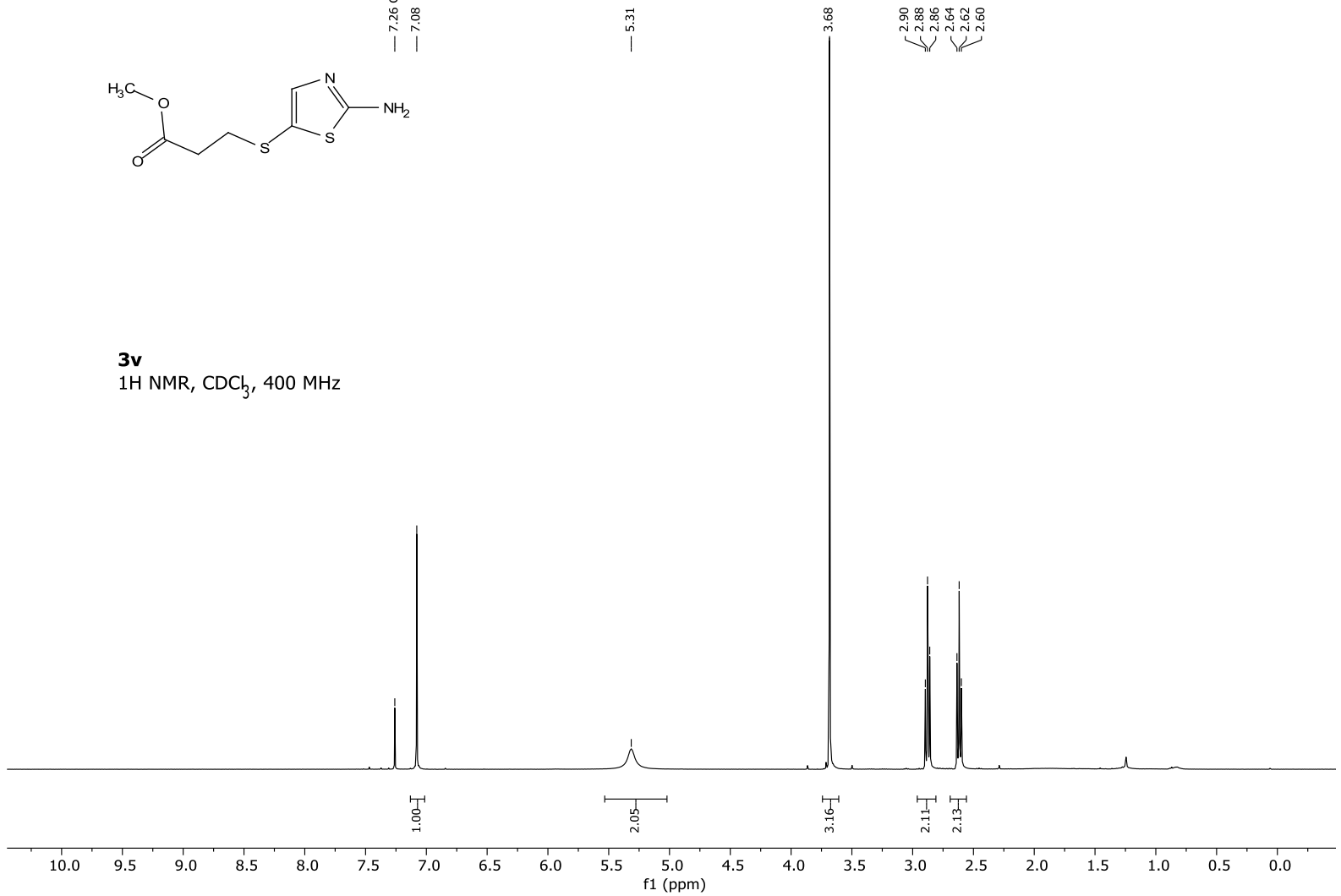<smiles>COC(=O)CCSc1cnc(N)s1</smiles>

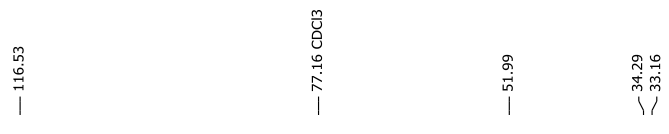

13C NMR, $\mathrm{CDCl}_{3}, 101 \mathrm{MHz}$

$200 \quad 190$
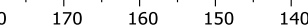

$130 \quad 120$

10100

$90 \quad 80$

70

50

30 
4-Chloro-5-(dodecylthio)-1-methyl-1H-imidazole (3w)

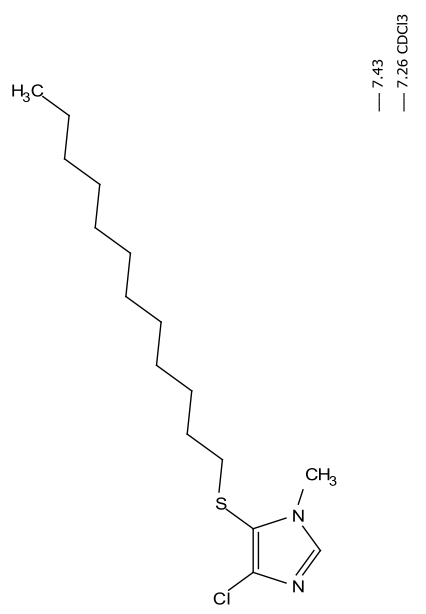

3w

$1 \mathrm{H} \mathrm{NMR}, \mathrm{CDCl}_{3}, 300 \mathrm{MHz}$

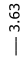

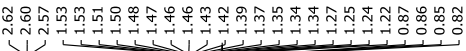
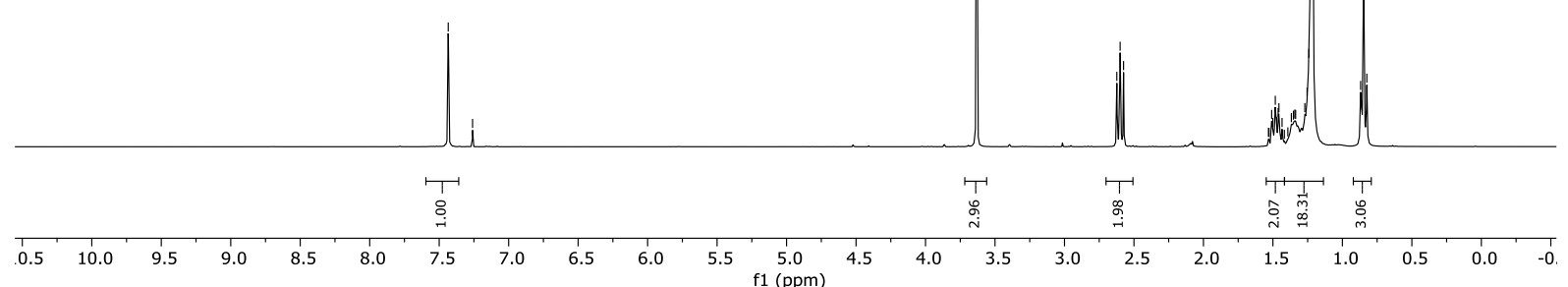

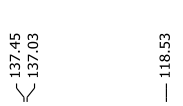

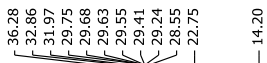

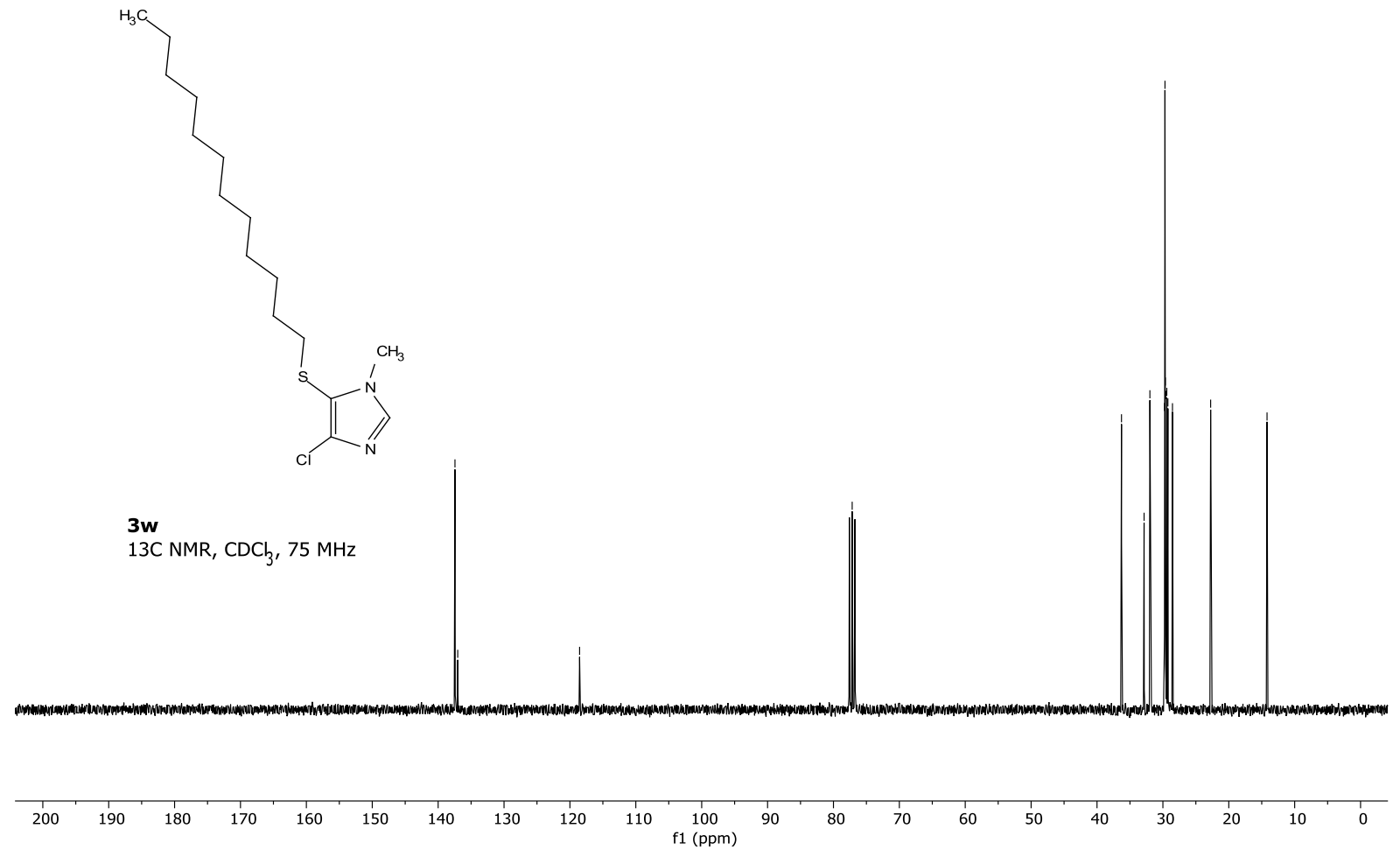


4-Chloro-5-(cyclohexylthio)-1-methyl-1H-imidazole (3x)

1.<smiles>Cn1cnc(Cl)c1SC1CCCCC1</smiles>

$1 \mathrm{H} N M R, C D C l, 400 \mathrm{MHz}$

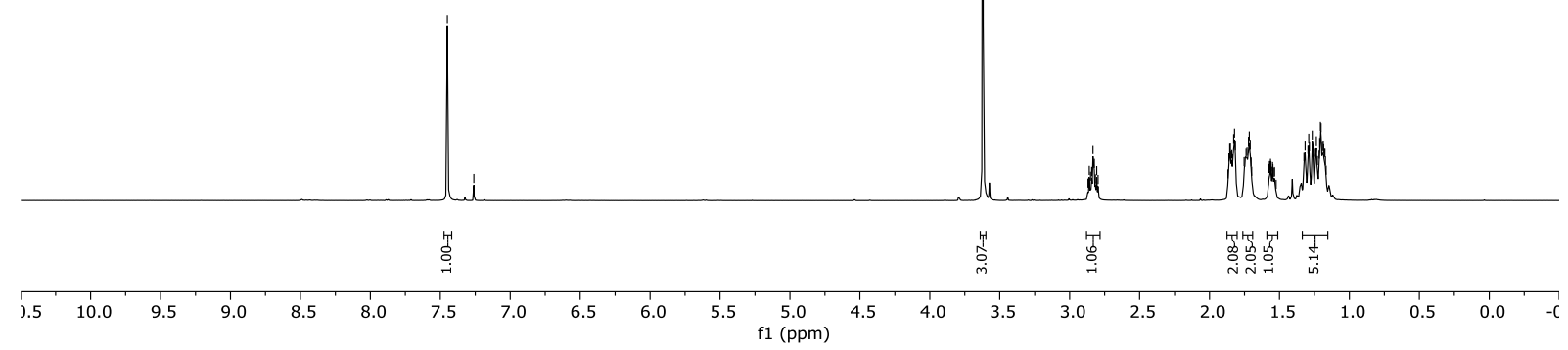<smiles>Cn1cnc(Cl)c1SC1CCCCC1</smiles>

13. NMR, $\mathrm{CDCl}_{3}, 101 \mathrm{MHz}$

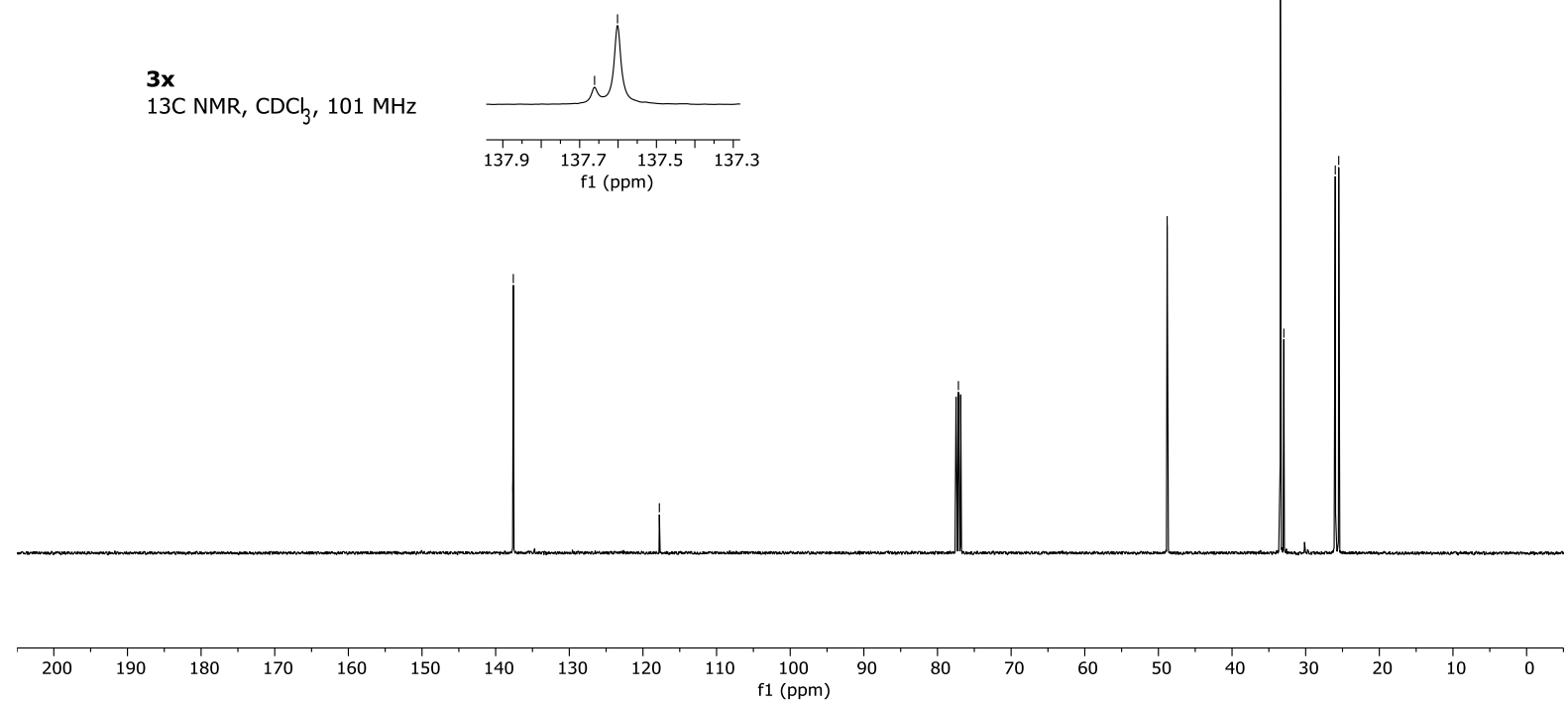


5-(Benzylthio)-4-chloro-1-methyl-1 $H$-imidazole (3y)
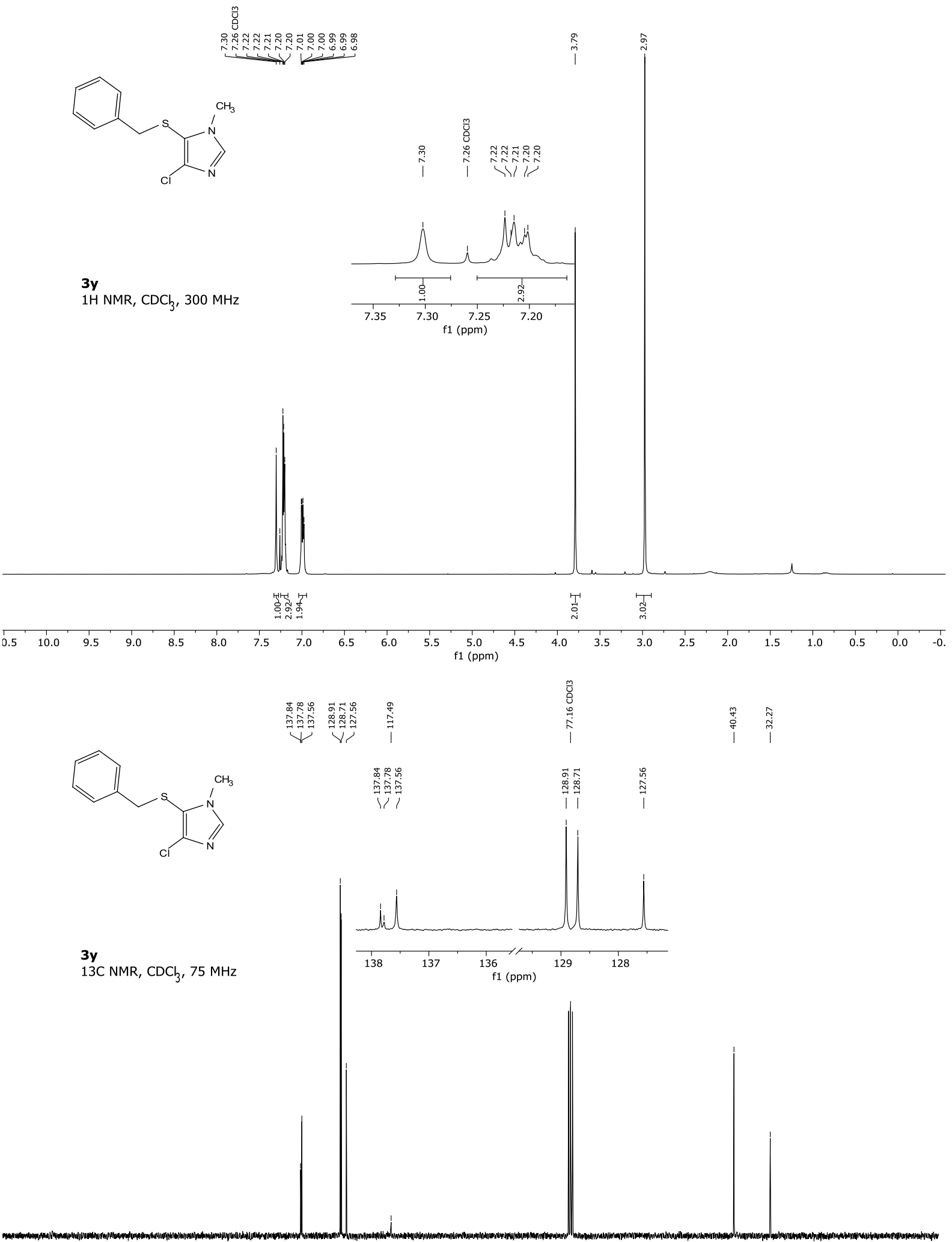

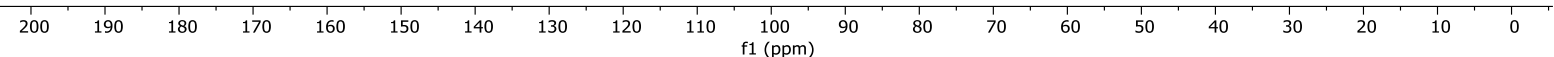


2-((4-Chloro-1-methyl-1H-imidazol-5-yl)thio)ethan-1-ol (3z)
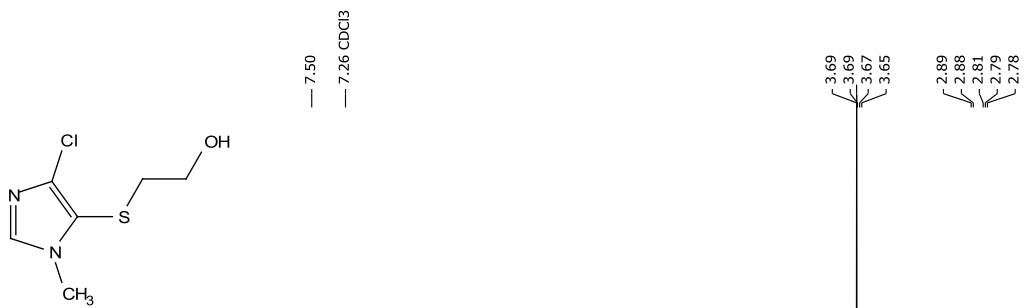

$3 z$

$1 \mathrm{H} N \mathrm{MR}, \mathrm{CDCl}_{3}, 400 \mathrm{MHz}$
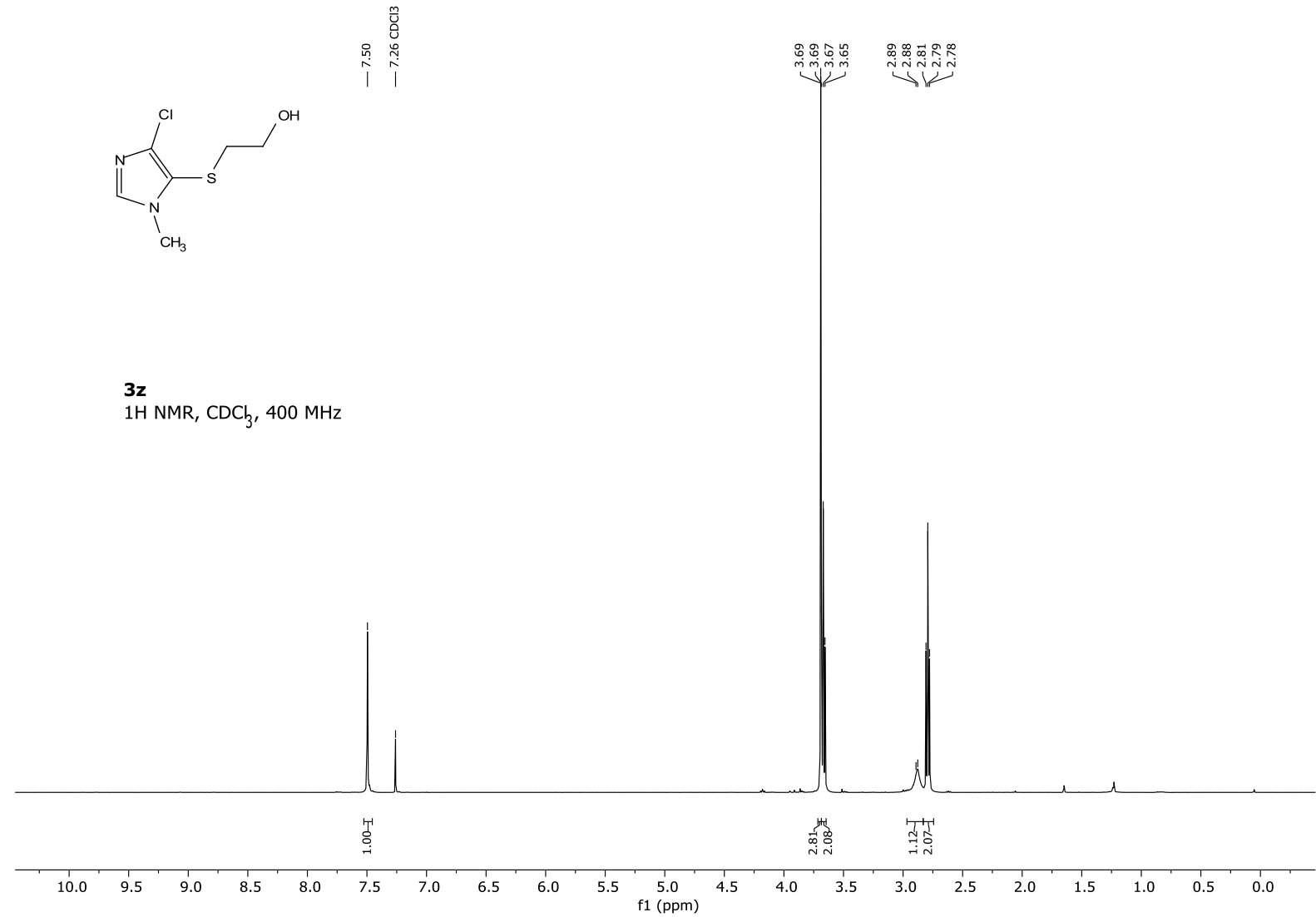

13C NMR, $\mathrm{CDCl}_{3}, 101 \mathrm{MHz}$
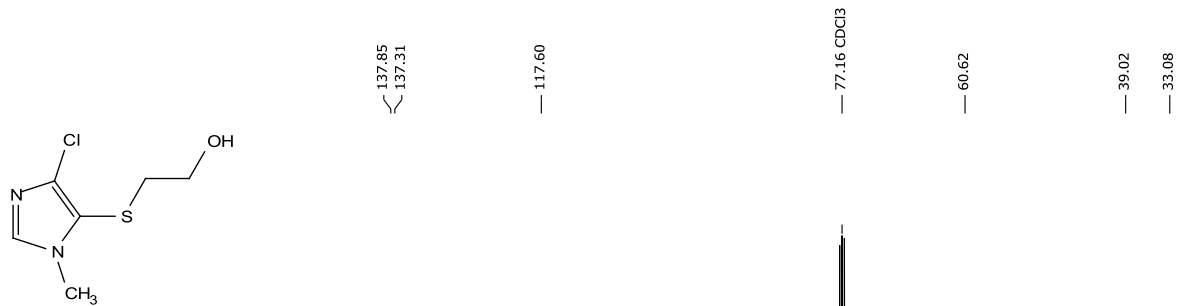

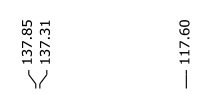

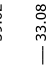

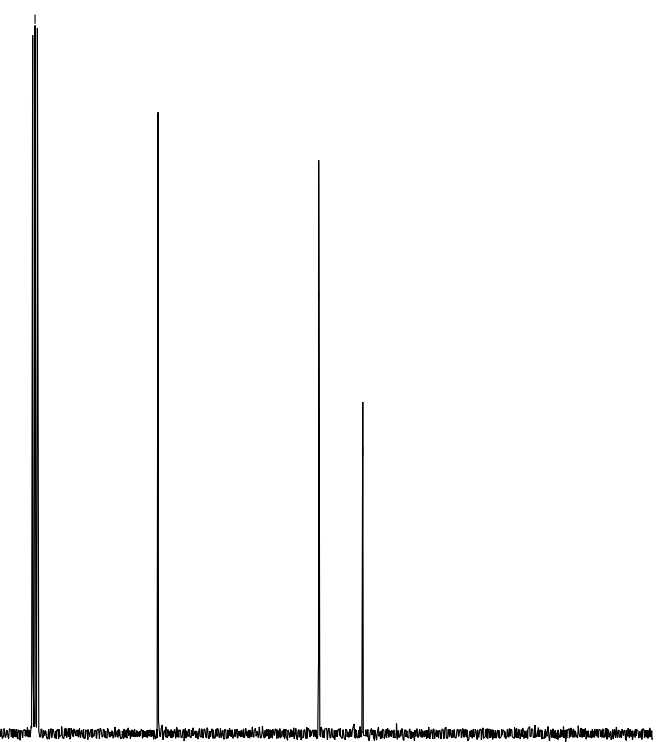


4-Chloro-5-((3-chloropropyl)thio)-1-methyl-1H-imidazole (3aa)

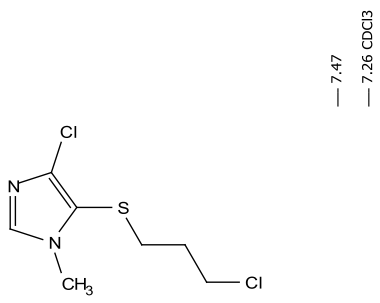

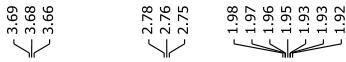

3aa

$1 \mathrm{H} \mathrm{NMR,} \mathrm{CDCl}_{3}, 400 \mathrm{MHz}$

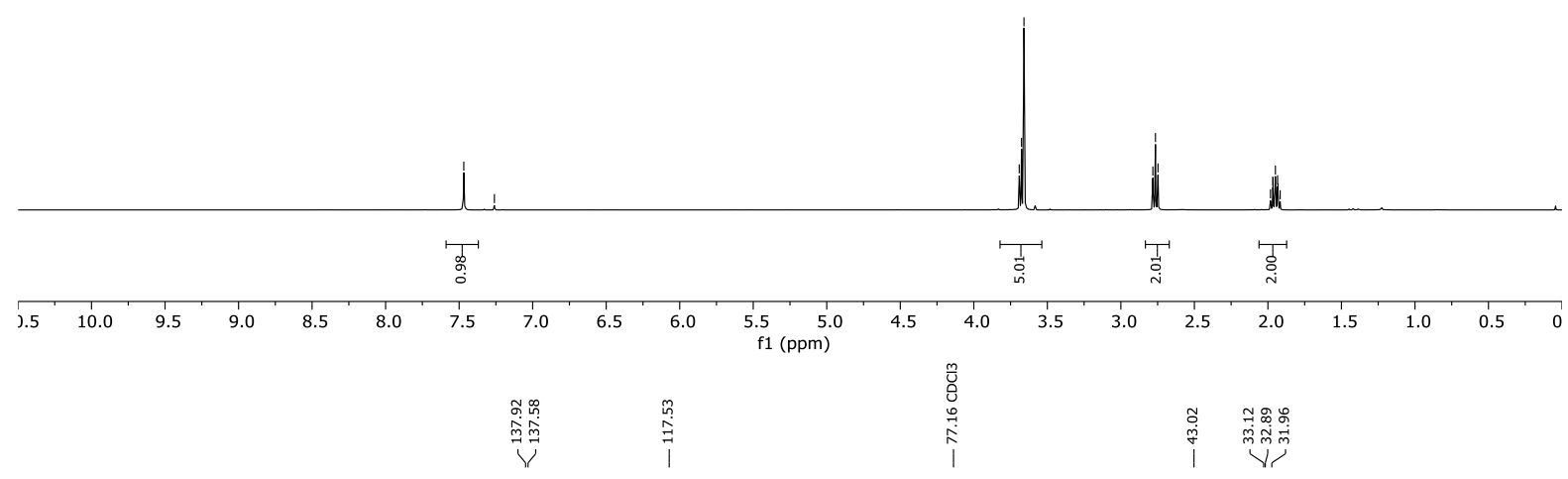

3aa

$13 \mathrm{C} \mathrm{NMR}, \mathrm{CDCl}_{3}, 101 \mathrm{MHz}$

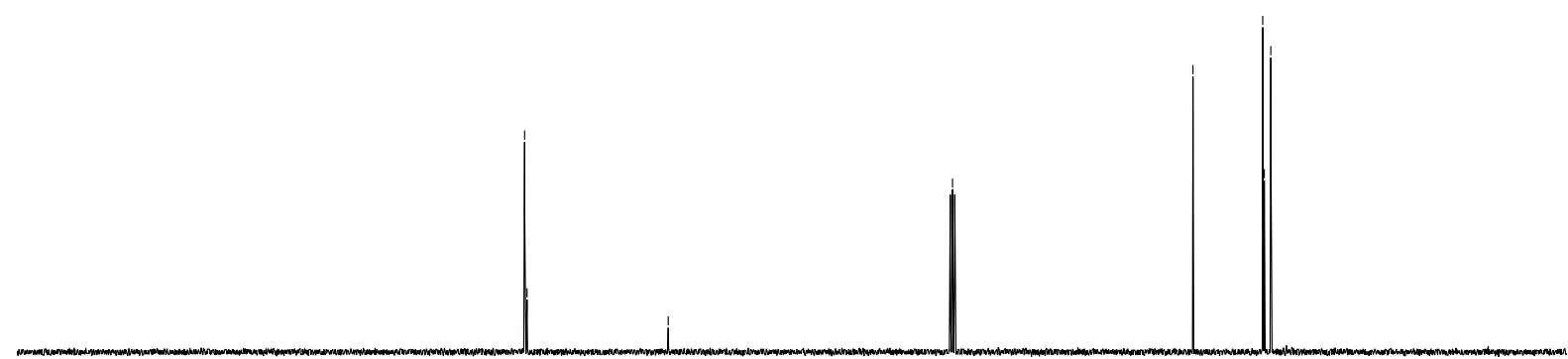

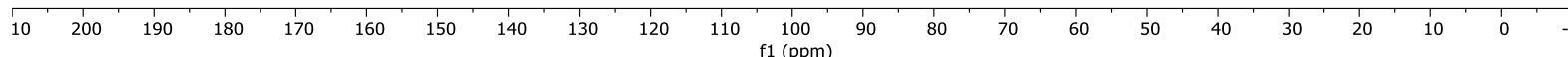


1,3-Bis((4-chloro-1-methyl-1H-imidazol-5-yl)thio)propane (3ab)

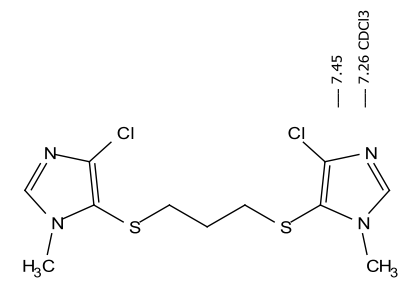

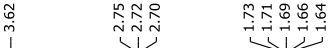

$3 a b$

$1 \mathrm{H} \mathrm{NMR}, \mathrm{CDCl}_{3}, 300 \mathrm{MHz}$

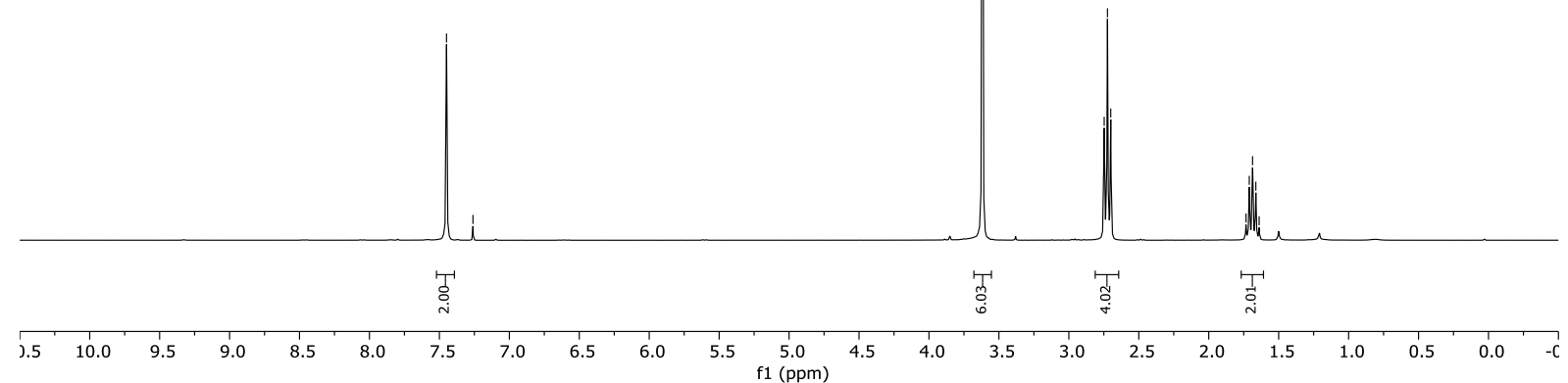<smiles>Cn1cnc(Cl)c1SCCCSc1c(Cl)ncn1C</smiles>

$13 \mathrm{C}$ NMR, $\mathrm{CDCl}_{3}, 75 \mathrm{MHz}$ 
Methyl $N$-(tert-butoxycarbonyl)-S-(4-chloro-1-methyl-1 $H$-imidazol-5-yl)- $L$-cysteinate (3ac)
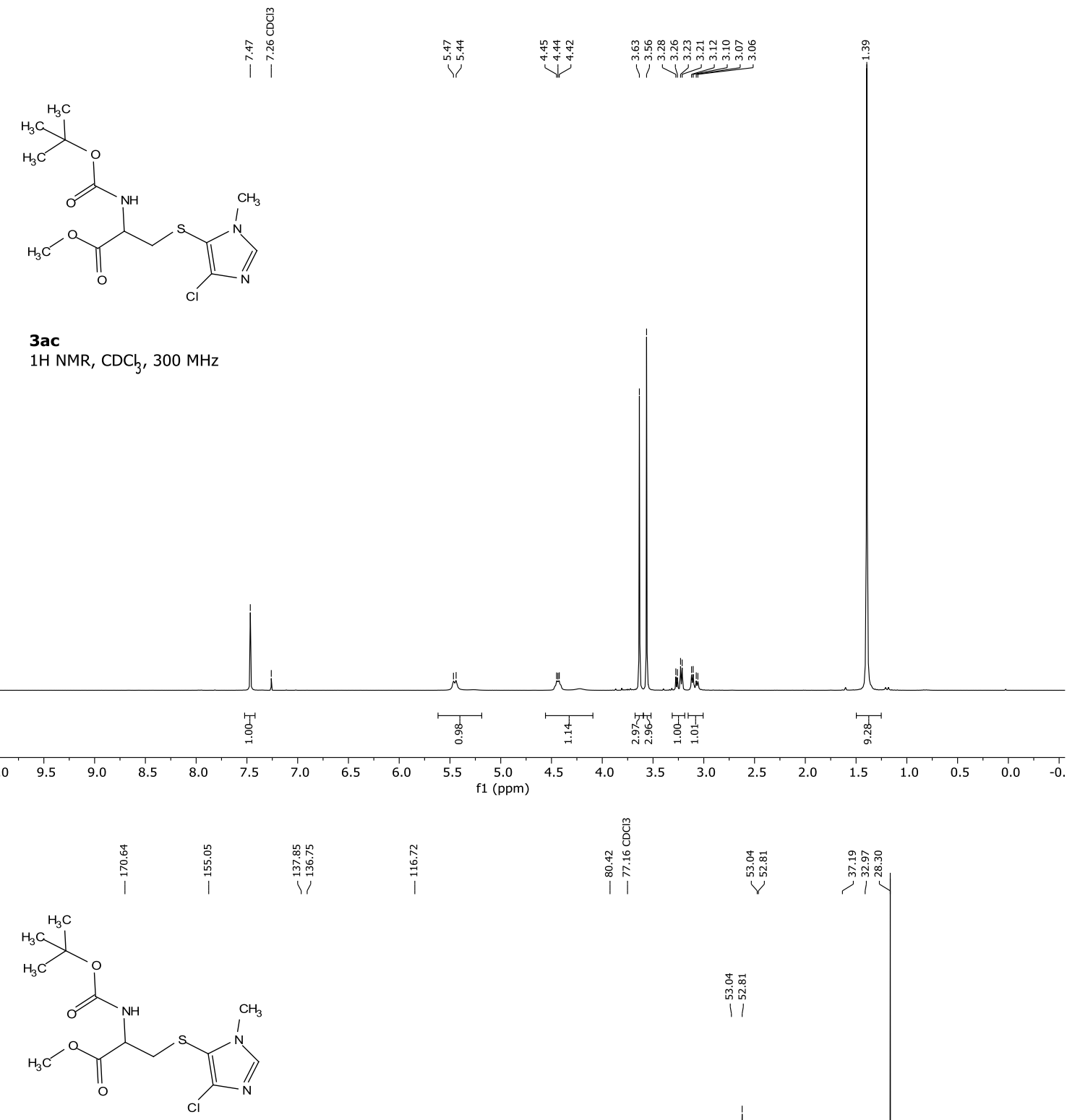

$13 \mathrm{C}$ NMR, $\mathrm{CDCl}_{3}, 75 \mathrm{MHz}$
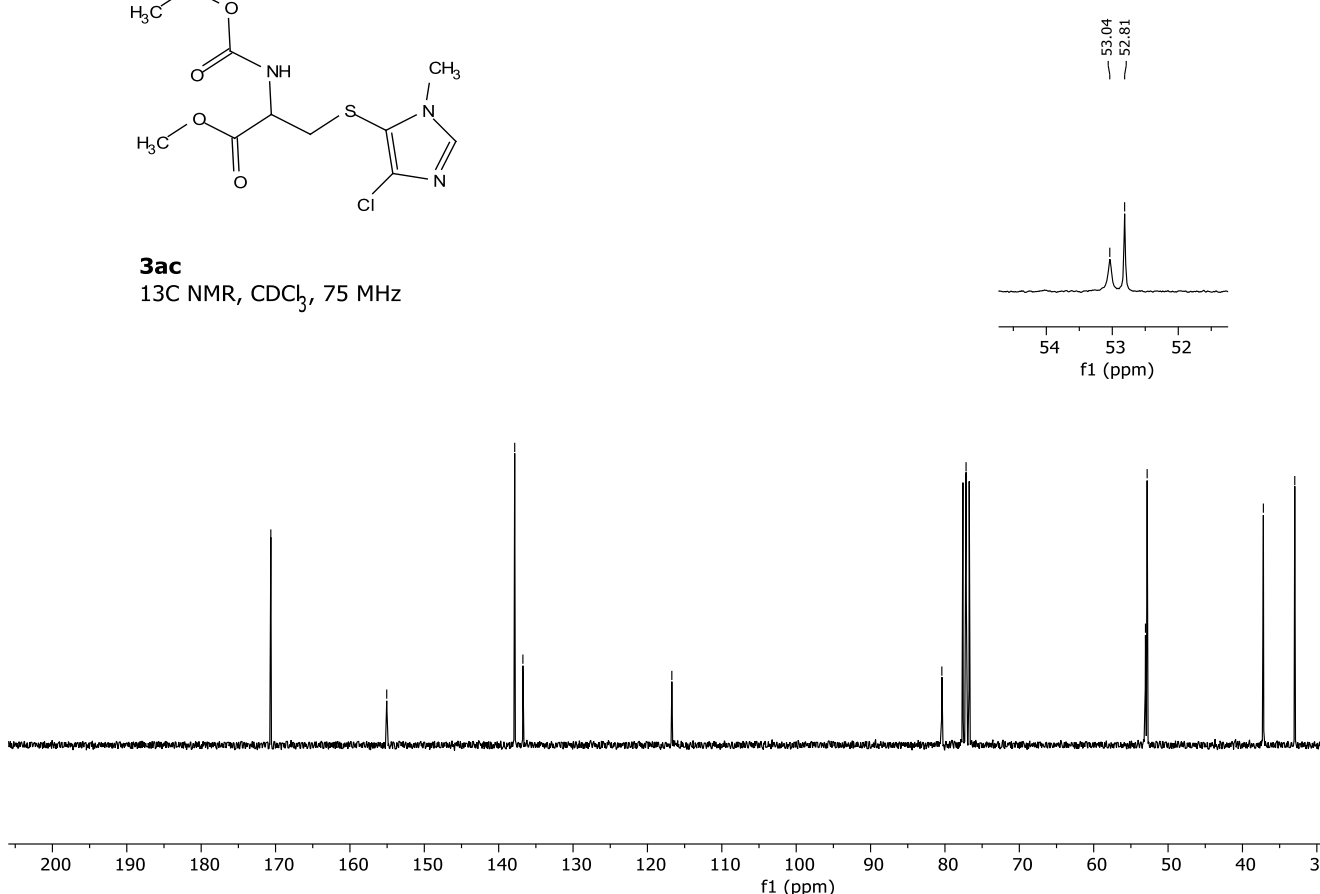

$\begin{array}{lllll}1 & 1 & 1 & 1 & 1 \\ 40 & 20 & 10 & 0\end{array}$ 
4-Chloro-1-methyl-5-((4-(trifluoromethyl)phenyl)thio)-1H-imidazole (3ad)
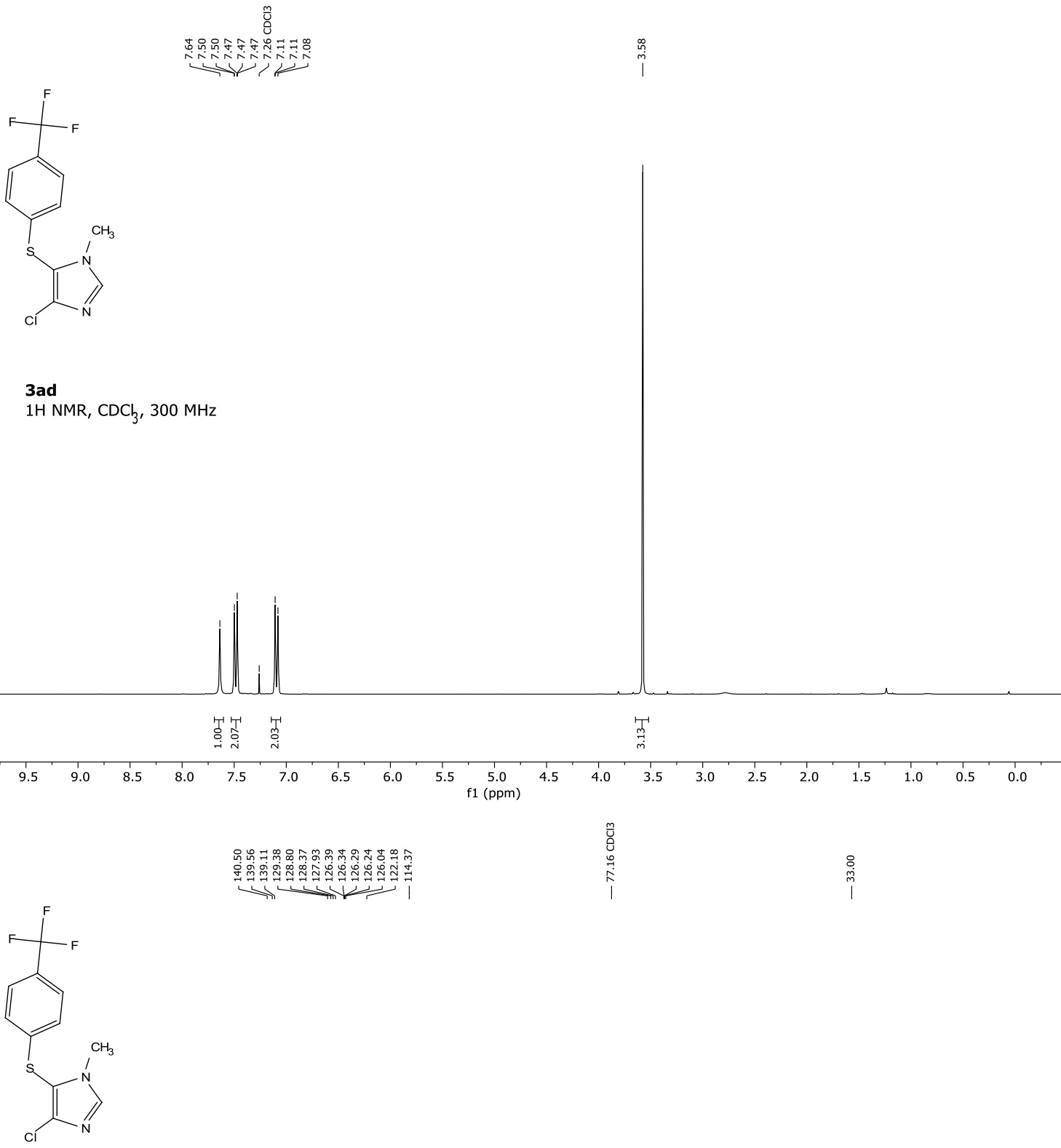

3ad

13C NMR, $\mathrm{CDCl}_{3}, 75 \mathrm{MHz}$

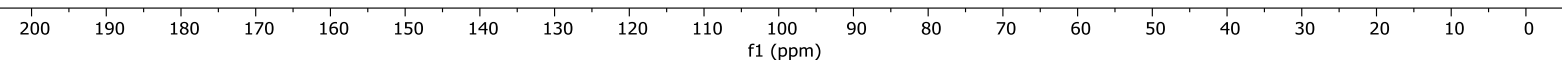




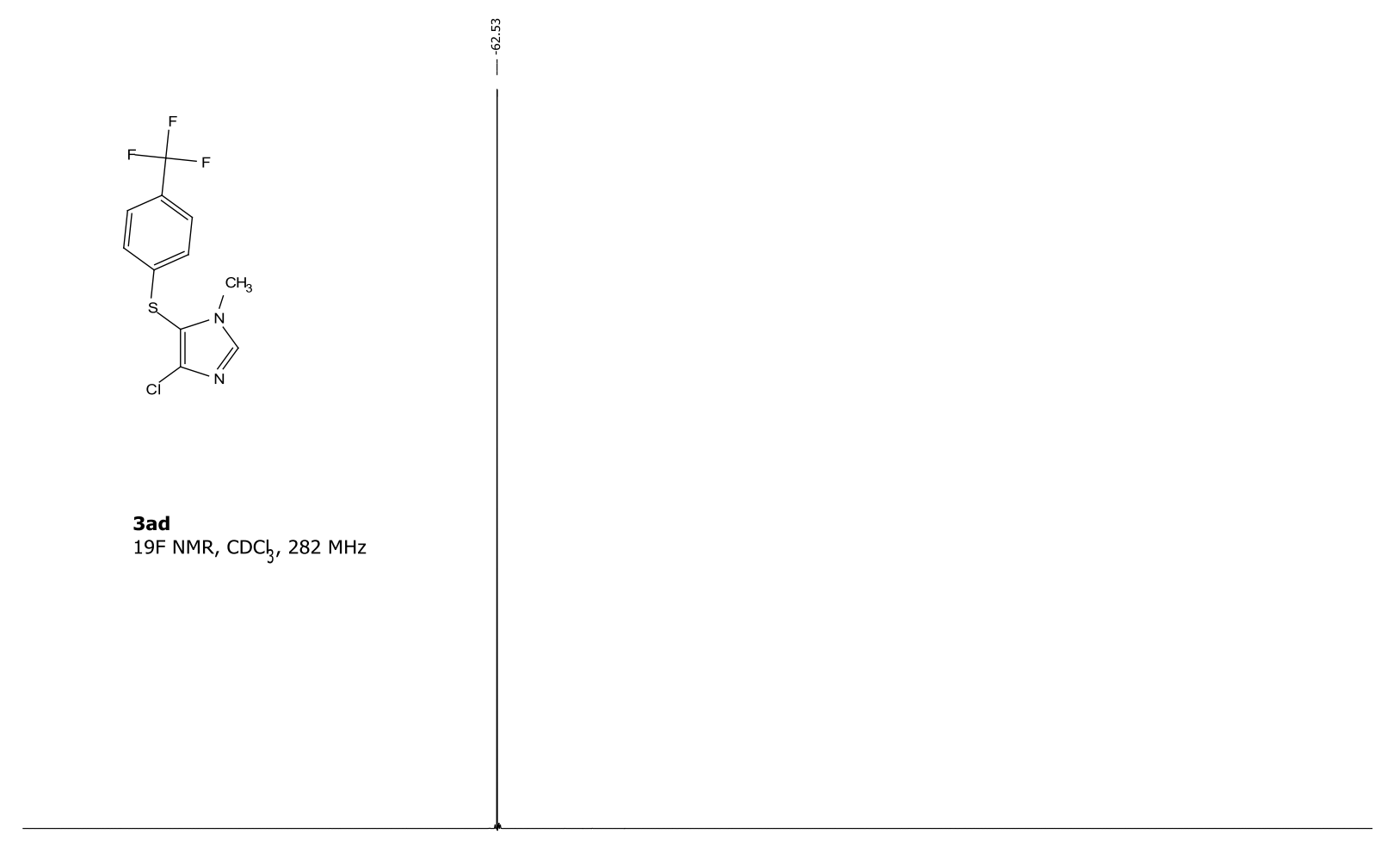

\begin{tabular}{llllllllllllllllllllllllllllllll}
\hline 20 & 10 & 0 & -10 & -20 & -30 & -40 & -50 & -60 & -70 & -80 & -90 & -100 & -110 & -120 & -130 & -140 & -150 & -160 & -170 & -180 & -190 & -100 & -210 & -220 \\
\hline
\end{tabular} 
4-Chloro-5-((2,3-dichlorophenyl)thio)-1-methyl-1H-imidazole (3ae)

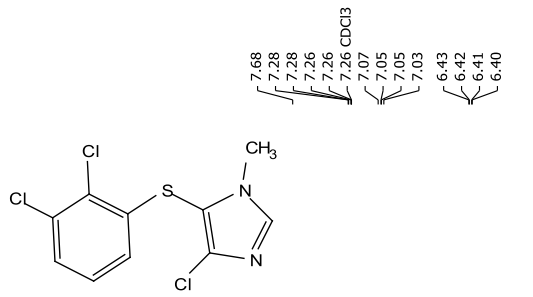

$1 \mathrm{H} \mathrm{NMR}, \mathrm{CDCl}, 400 \mathrm{MHz}$
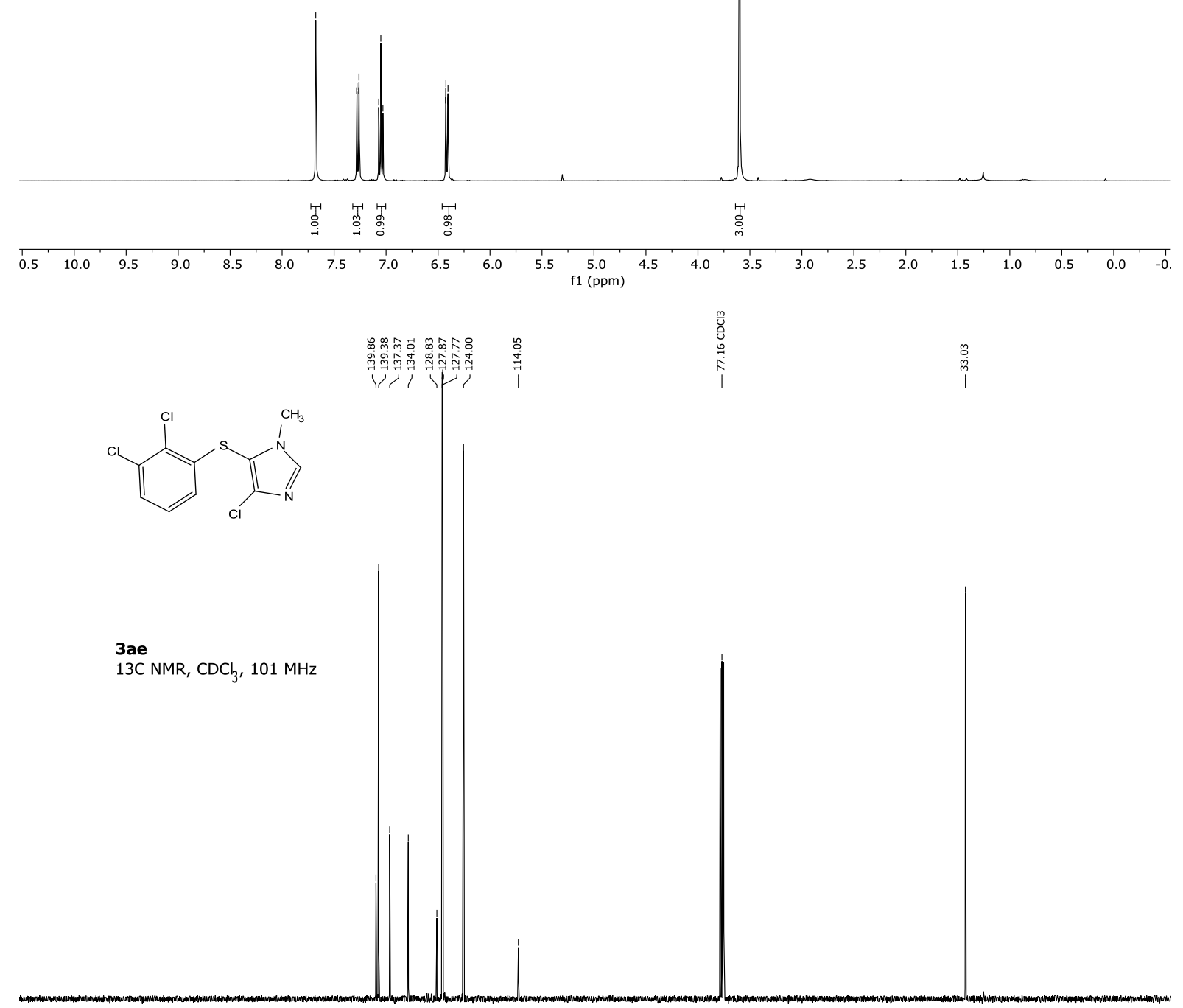

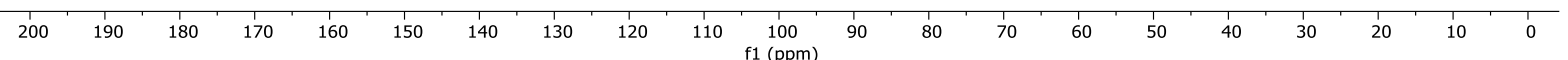


Methyl 2-((4-chloro-1-methyl-1H-imidazol-5-yl)thio)benzoate (3af)
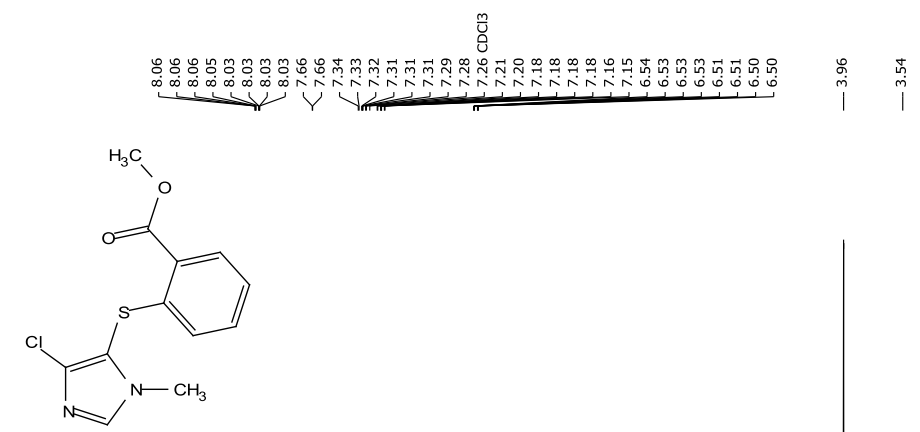

3 af

$1 \mathrm{H} \mathrm{NMR}, \mathrm{CDCl}_{3}, 300 \mathrm{MHz}$
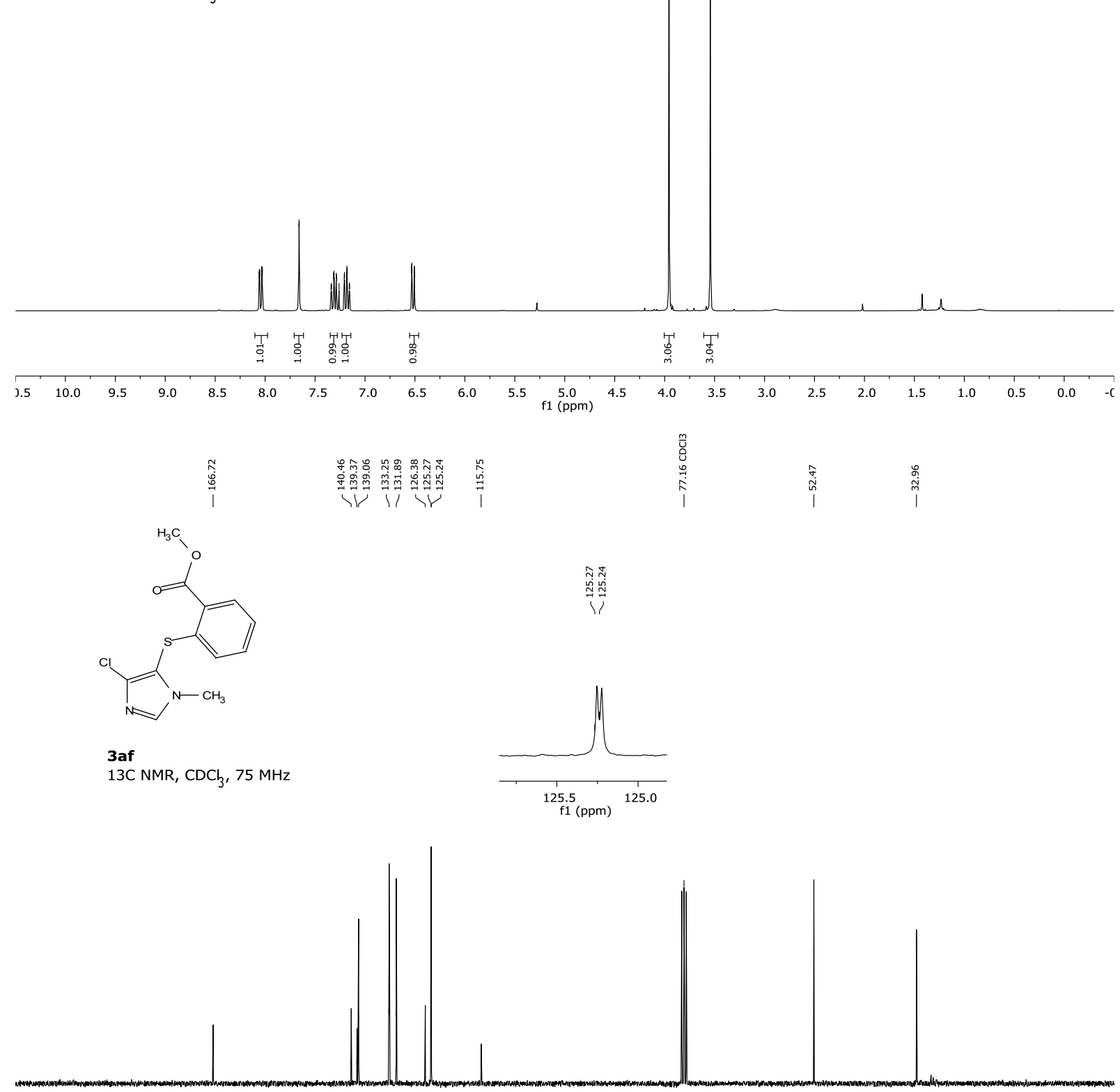

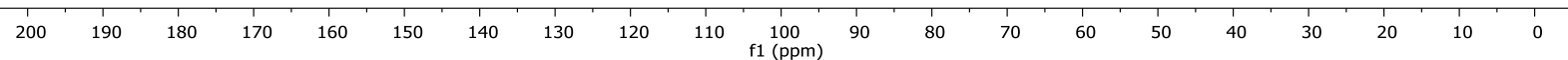


4-Chloro-1-methyl-5-(phenylthio)-1H-imidazole (3ag)

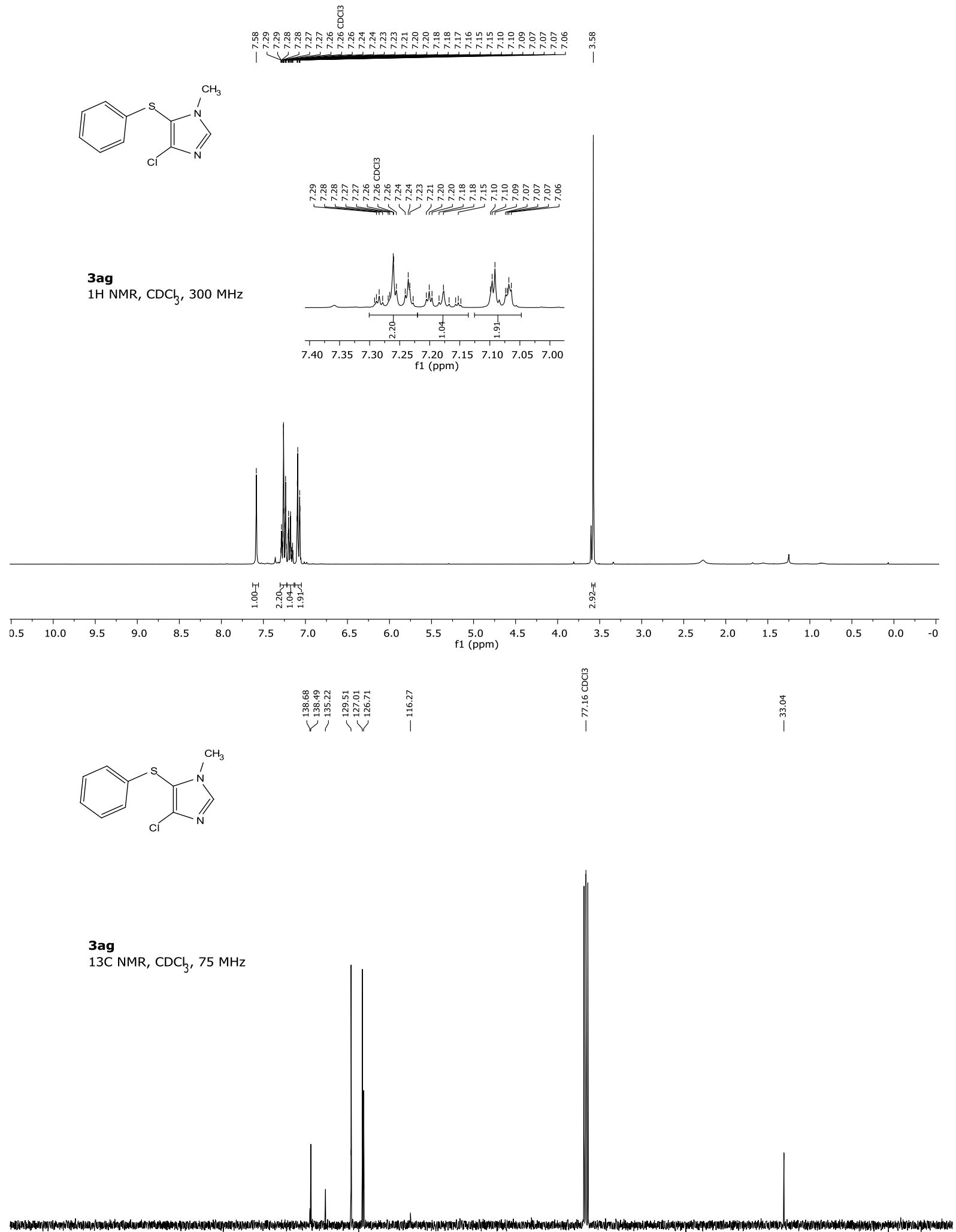

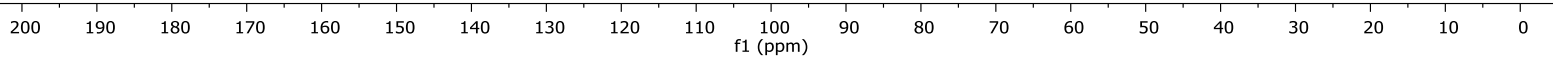


4-Chloro-1-methyl-5-(p-tolylthio)-1H-imidazole (3ah)
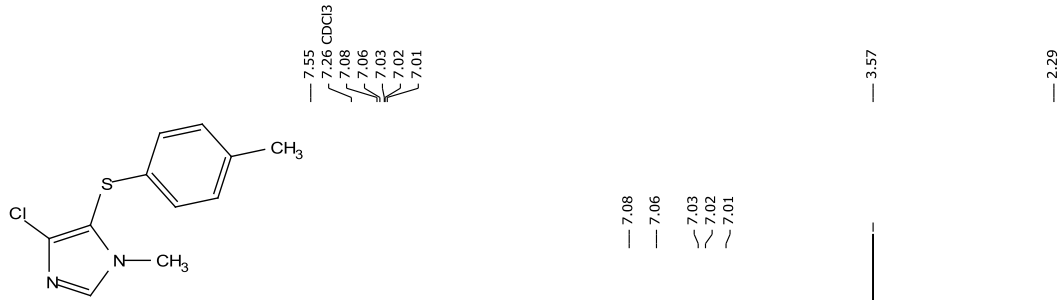

3ah

$1 \mathrm{H} \mathrm{NMR}, \mathrm{CDCl}_{3}, 400 \mathrm{MHz}$
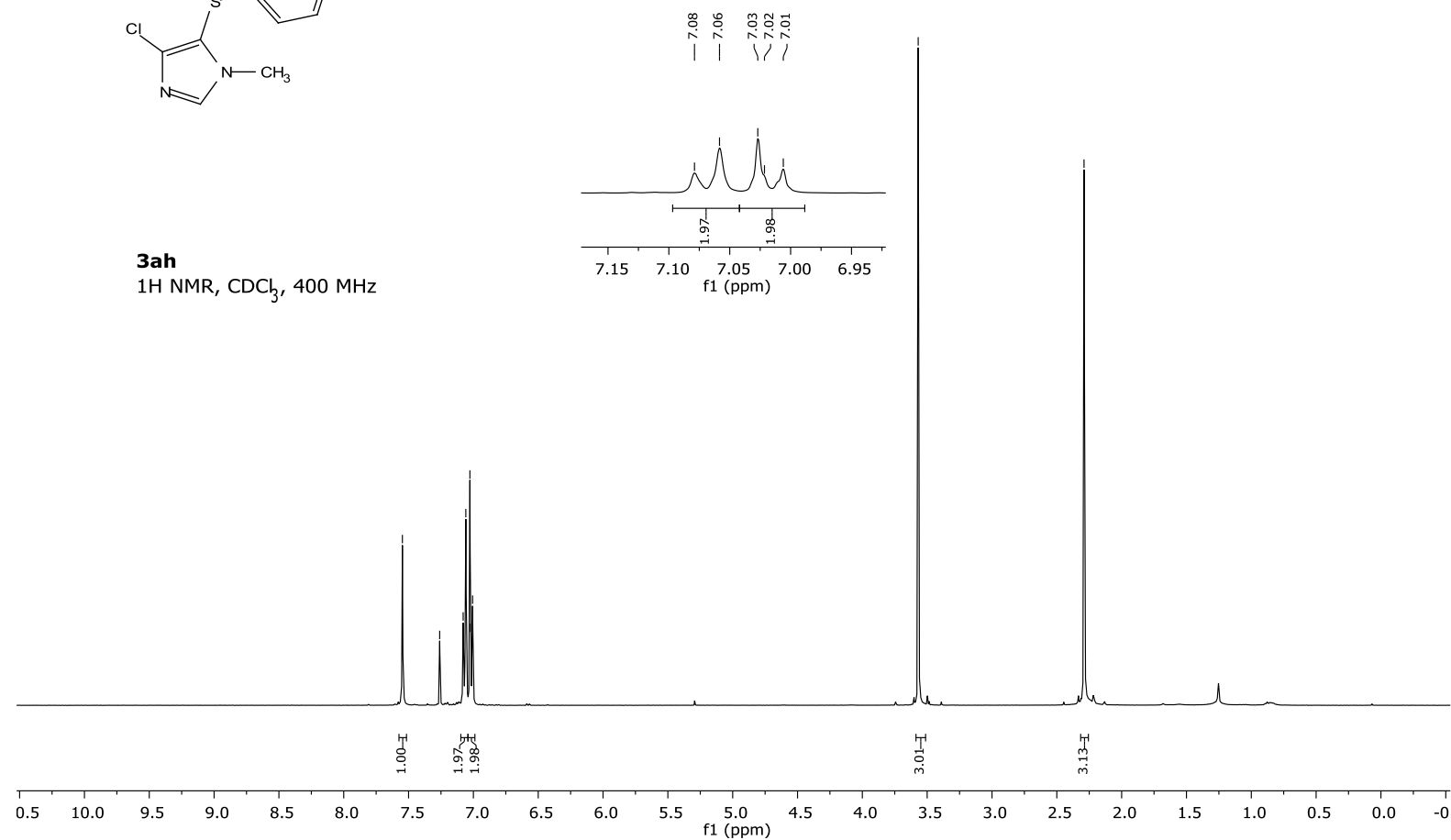

|

$m$
0
0
0
0
$i$
$i$

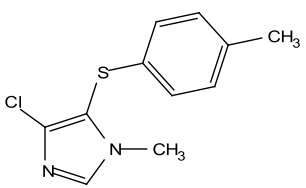

3ah

13C NMR, $\mathrm{CDCl}_{3}, 101 \mathrm{MHz}$

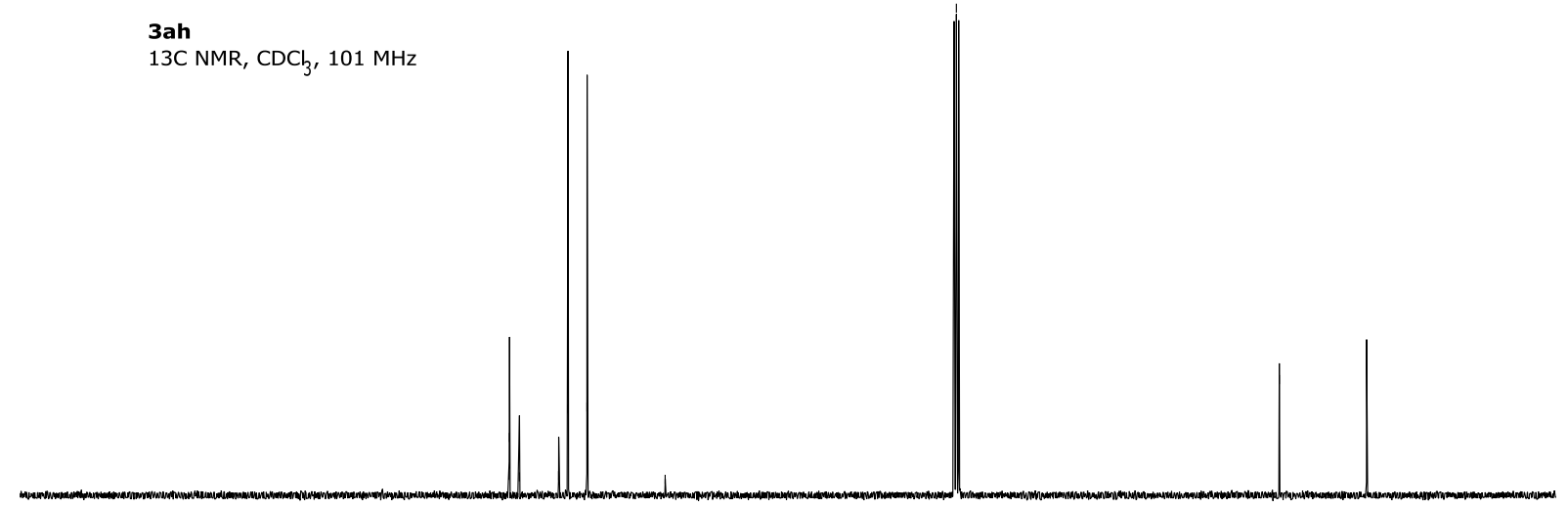

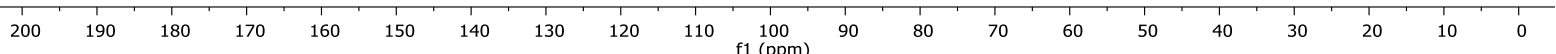


$N$-(4-((4-chloro-1-methyl-1H-imidazol-5-yl)thio)phenyl)-2,2,2-trifluoroacetamide (3ai)

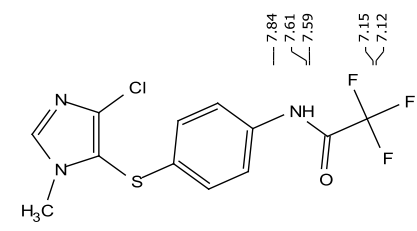

然

3ai

$1 \mathrm{H} \mathrm{NMR}, \mathrm{CD}_{3} \mathrm{OD}, 400 \mathrm{MHz}$

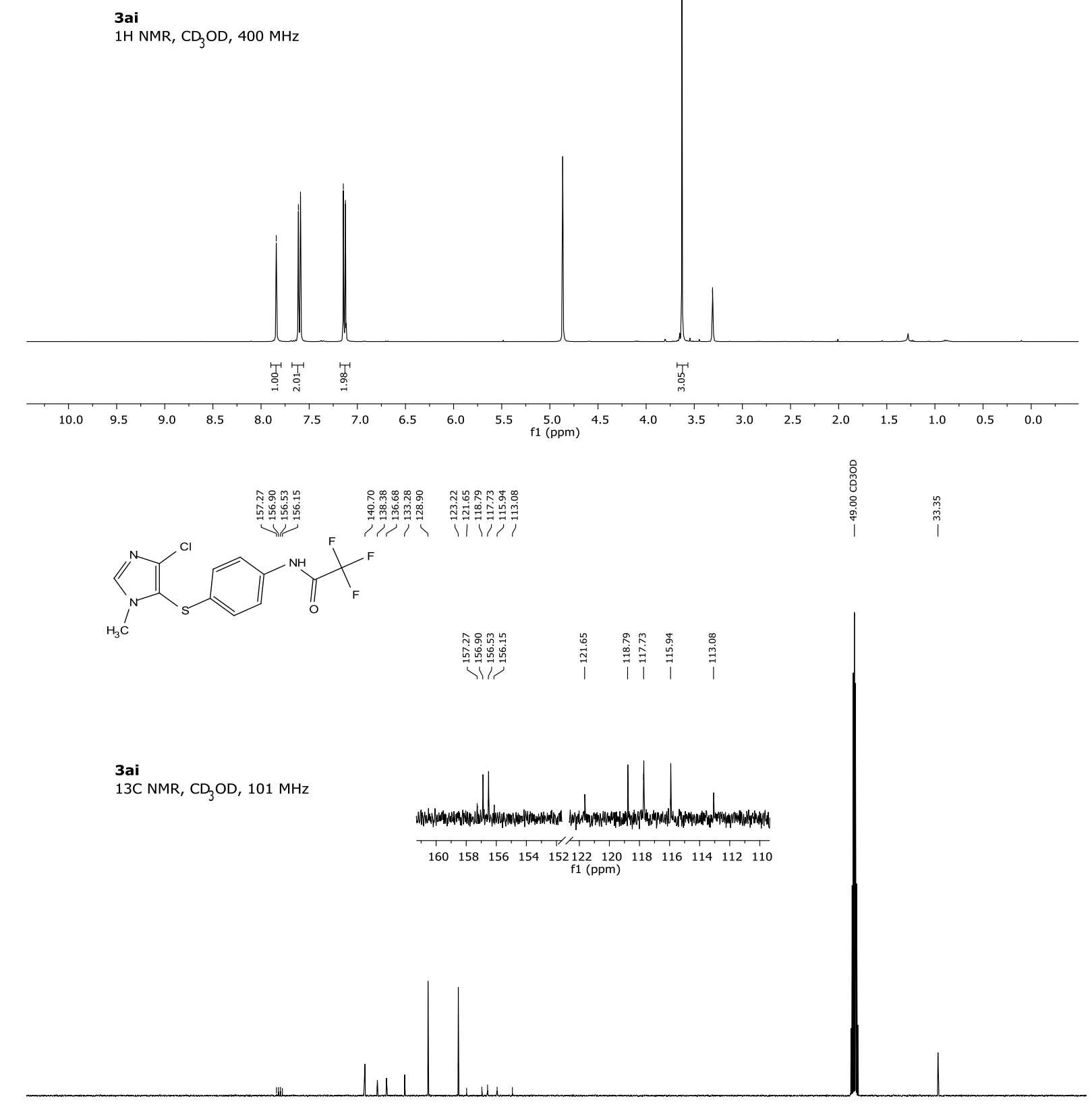

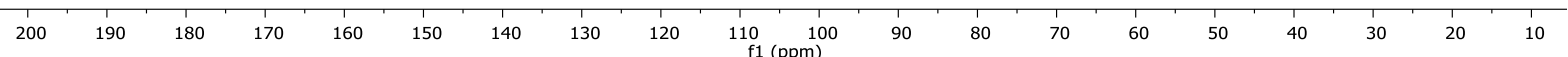


<smiles>Cn1cnc(Cl)c1Sc1ccc(NC(=O)C(F)(F)F)cc1</smiles>

3ai
19F NMR, $\mathrm{CD}_{3} \mathrm{OD}, 376 \mathrm{MHz}$

$\begin{array}{llllllllllllllllllllllllll}20 & 10 & 0 & -10 & -20 & -30 & -40 & -50 & -60 & -70 & -80 & -90 & -100 & -110 & -120 & -130 & -140 & -150 & -160 & -170 & -180 & -190 & -200 & -210 & -220\end{array}$ 
$N$-(2-((4-chloro-1-methyl-1H-imidazol-5-yl)thio)ethyl)-5-((3aS,4S,6a $R)-2$-oxohexahydro-1 $H$ thieno[3,4- $d$ ] imidazol-4-yl)pentanamide (3aj)

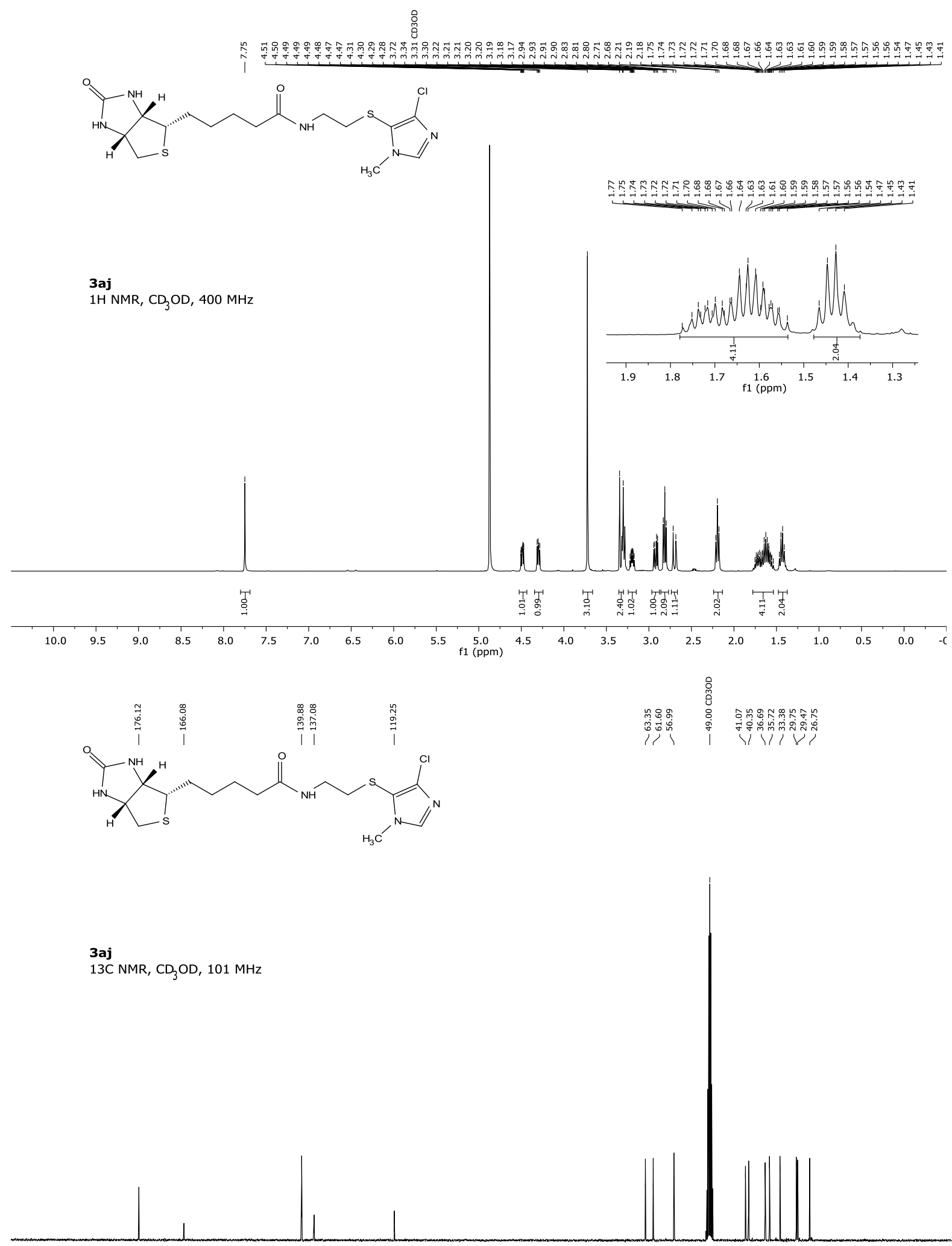

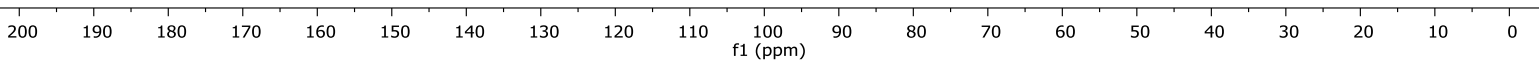


(2R,3R,4S,5R,6S)-2-(Acetoxymethyl)-6-((4-chloro-1-methyl-1H-imidazol-5yl)thio)tetrahydro-2 $H$-pyran-3,4,5-triyl triacetate (3ak)

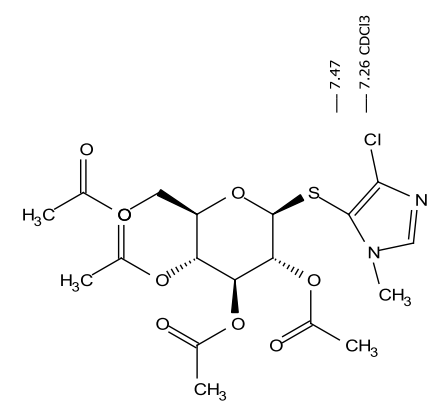

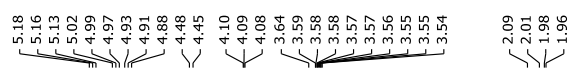

3ak

$1 \mathrm{H} \mathrm{NMR,} \mathrm{CDCl}_{3}, 400 \mathrm{MHz}$

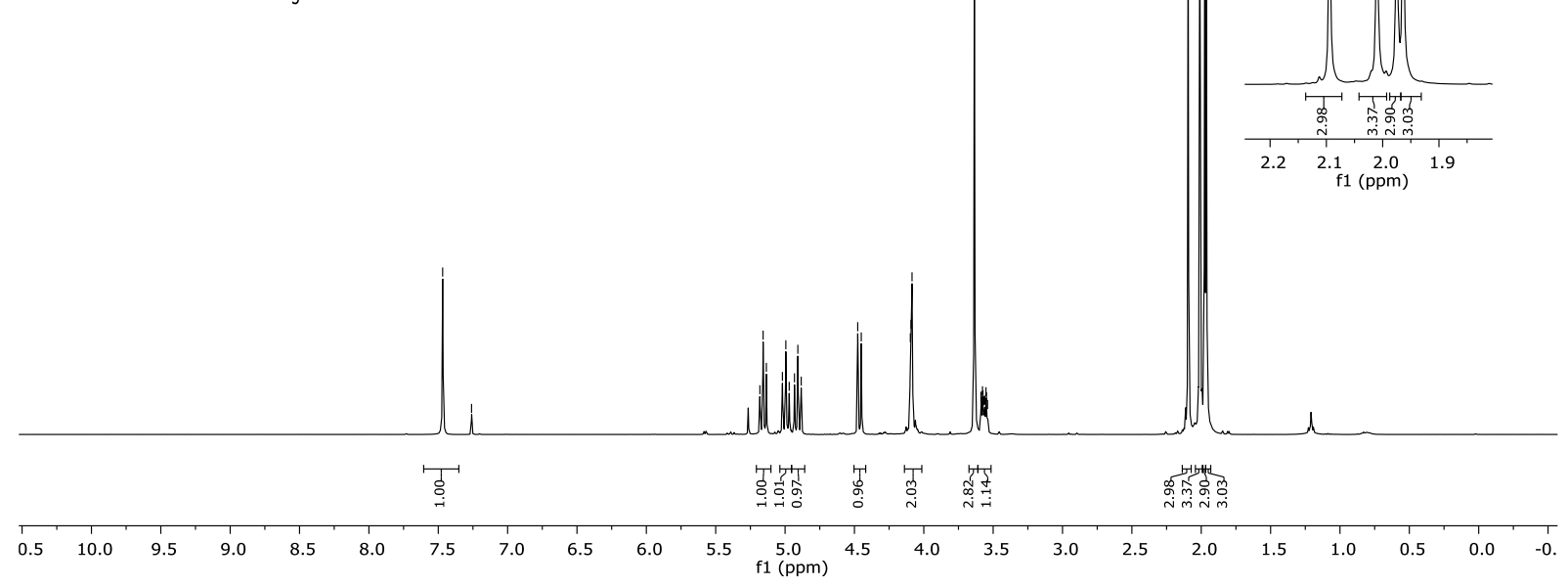<smiles>CC(=O)OC1C(Sc2c(Cl)ncn2C)OC2COC(C)=C(C)OC2C(OC(C)=O)C1OC(C)=O</smiles>

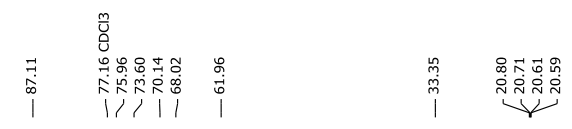

3ak

13C NMR, $\mathrm{CDCl}_{3}, 101 \mathrm{MHz}$

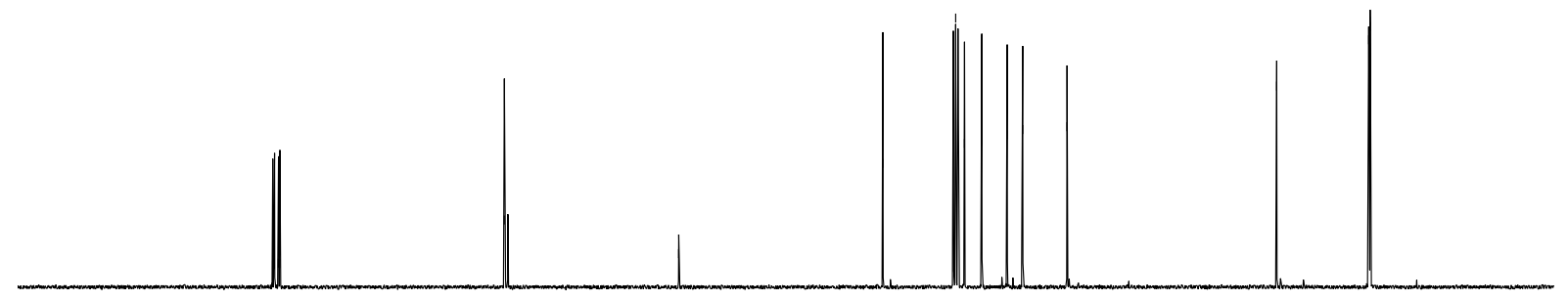


4-Chloro-5-(((3R,5S,8R,9S,10S,13R,14S,17R)-10,13-dimethyl-17-((R)-6-methylheptan-2yl)hexadecahydro-1H-cyclopenta[a]phenanthren-3-yl)thio)-1-methyl-1H-imidazole (3al) 范

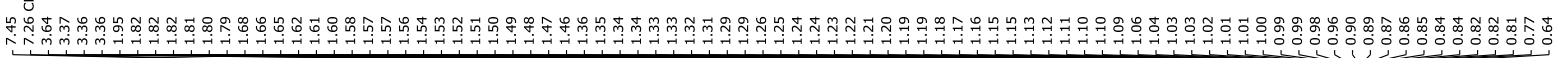

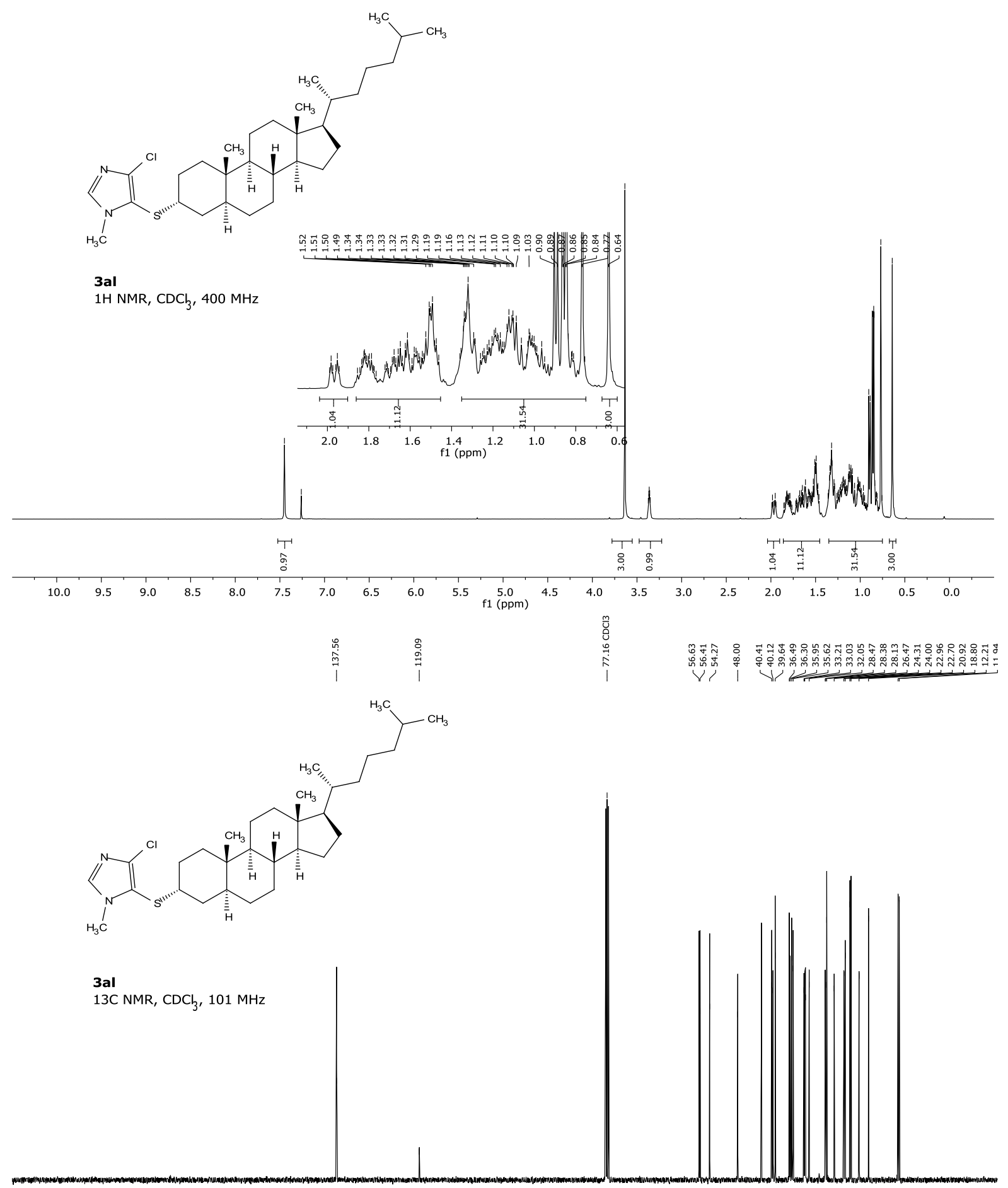

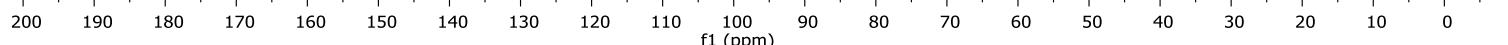


Methyl $\quad N^{2}$-(tert-butoxycarbonyl)- $N^{4}$-(2-((4-chloro-1-methyl-1H-imidazol-5-yl)thio)ethyl)- $L$ asparaginyl- $L$-phenylalaninate (3am)
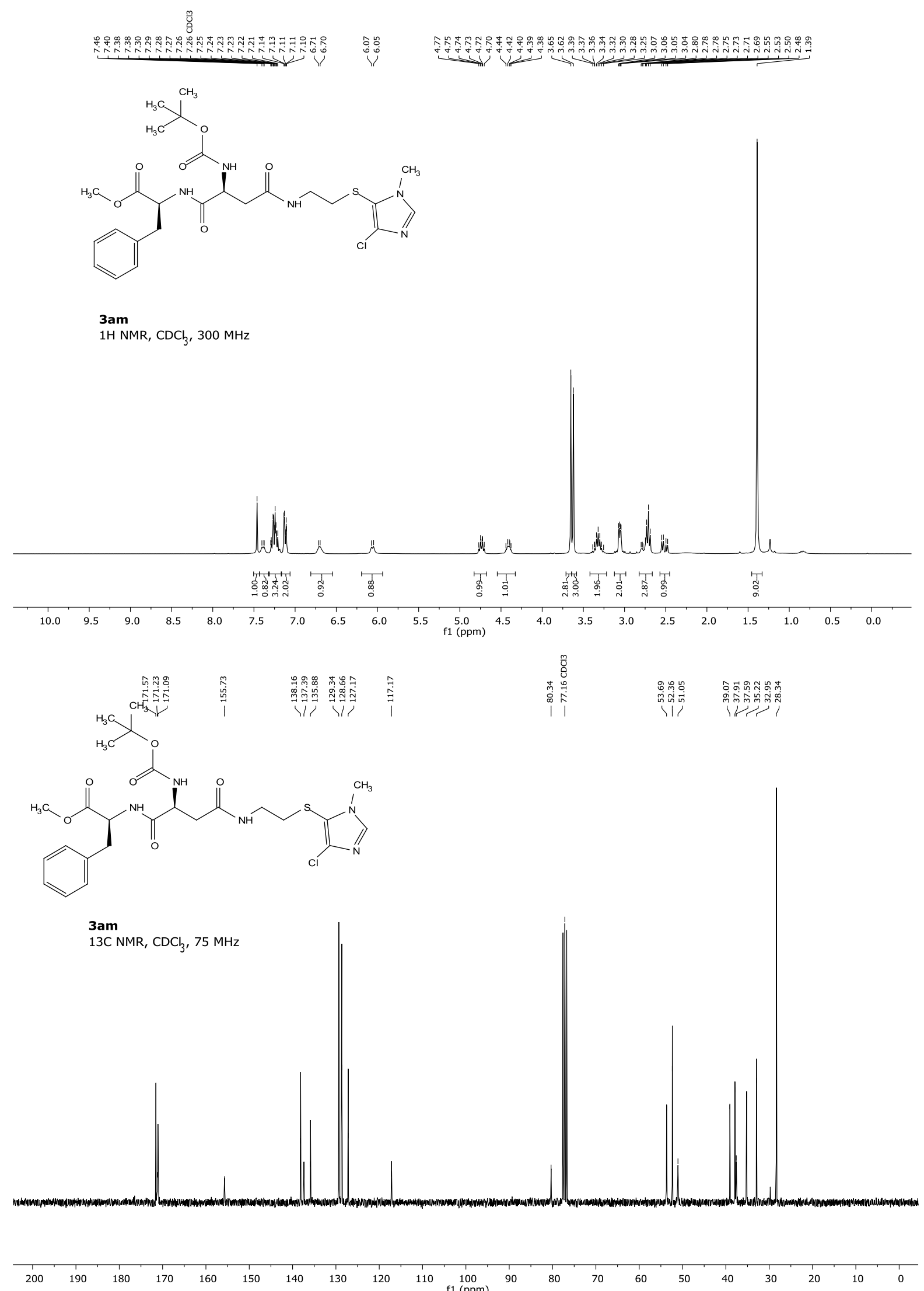
Methyl $N^{2}$-(tert-butoxycarbonyl)- $N^{5}$-((R)-3-((4-chloro-1-methyl-1H-imidazol-5-yl)thio)-1-((2methoxy-2-oxoethyl)amino)-1-oxopropan-2-yl)-L-glutaminate (3an)
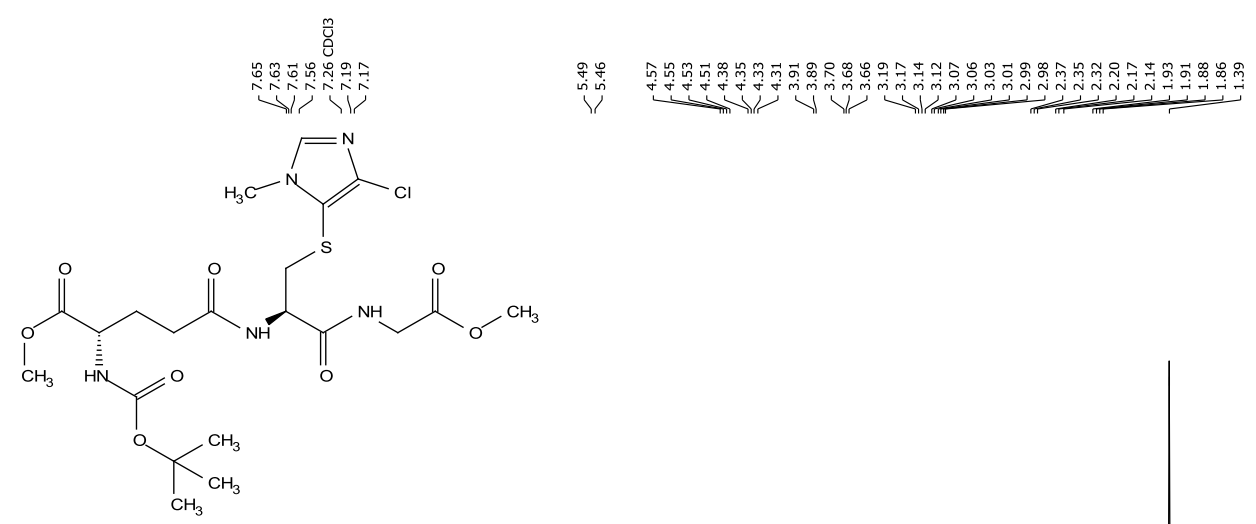

3an

$1 \mathrm{H} \mathrm{NMR}, \mathrm{CDCl}_{3}, 300 \mathrm{MHz}$
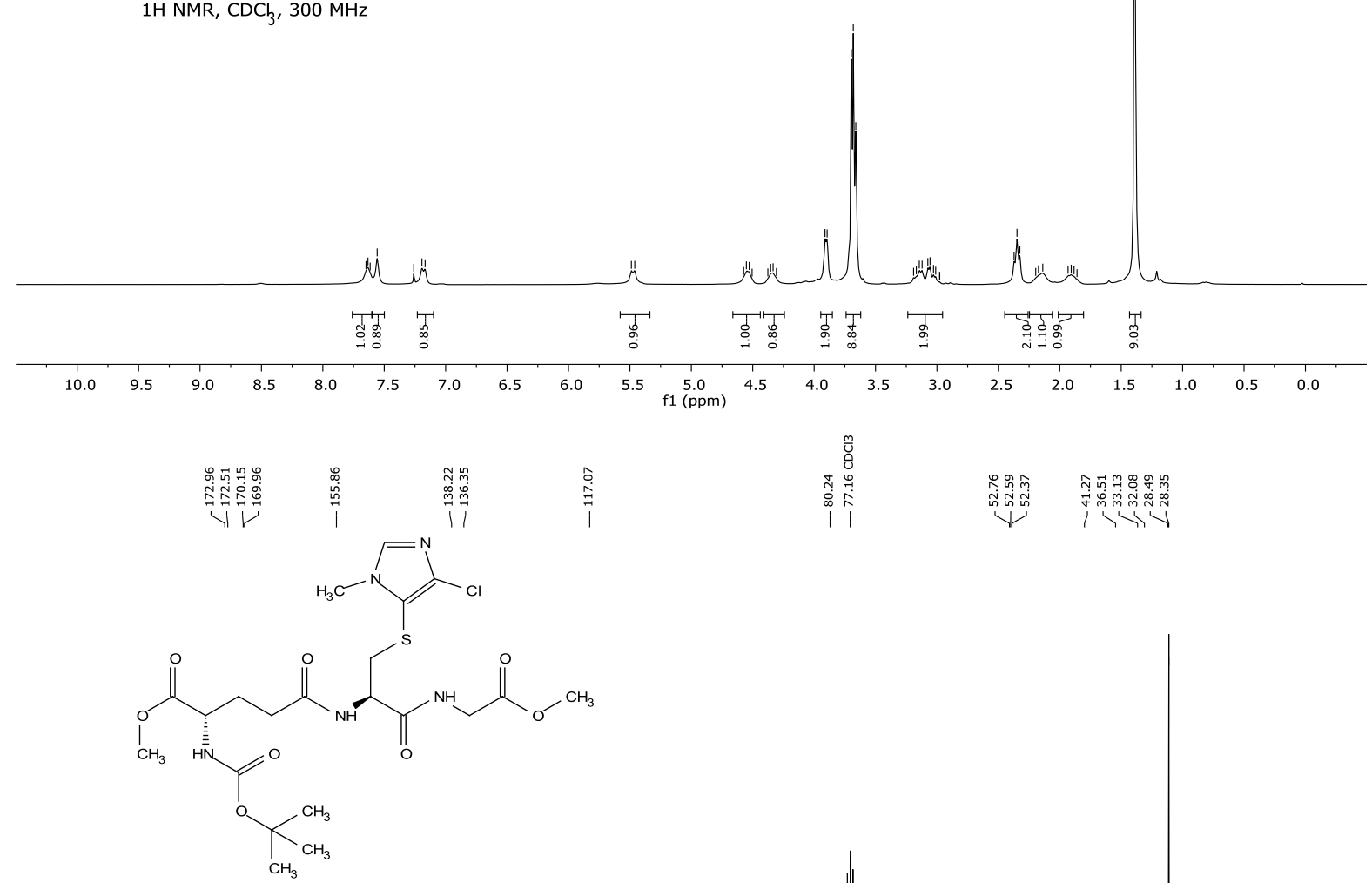

$3 a n$

$1 \mathrm{H} \mathrm{NMR,} \mathrm{CDCl}_{3}, 75 \mathrm{MHz}$
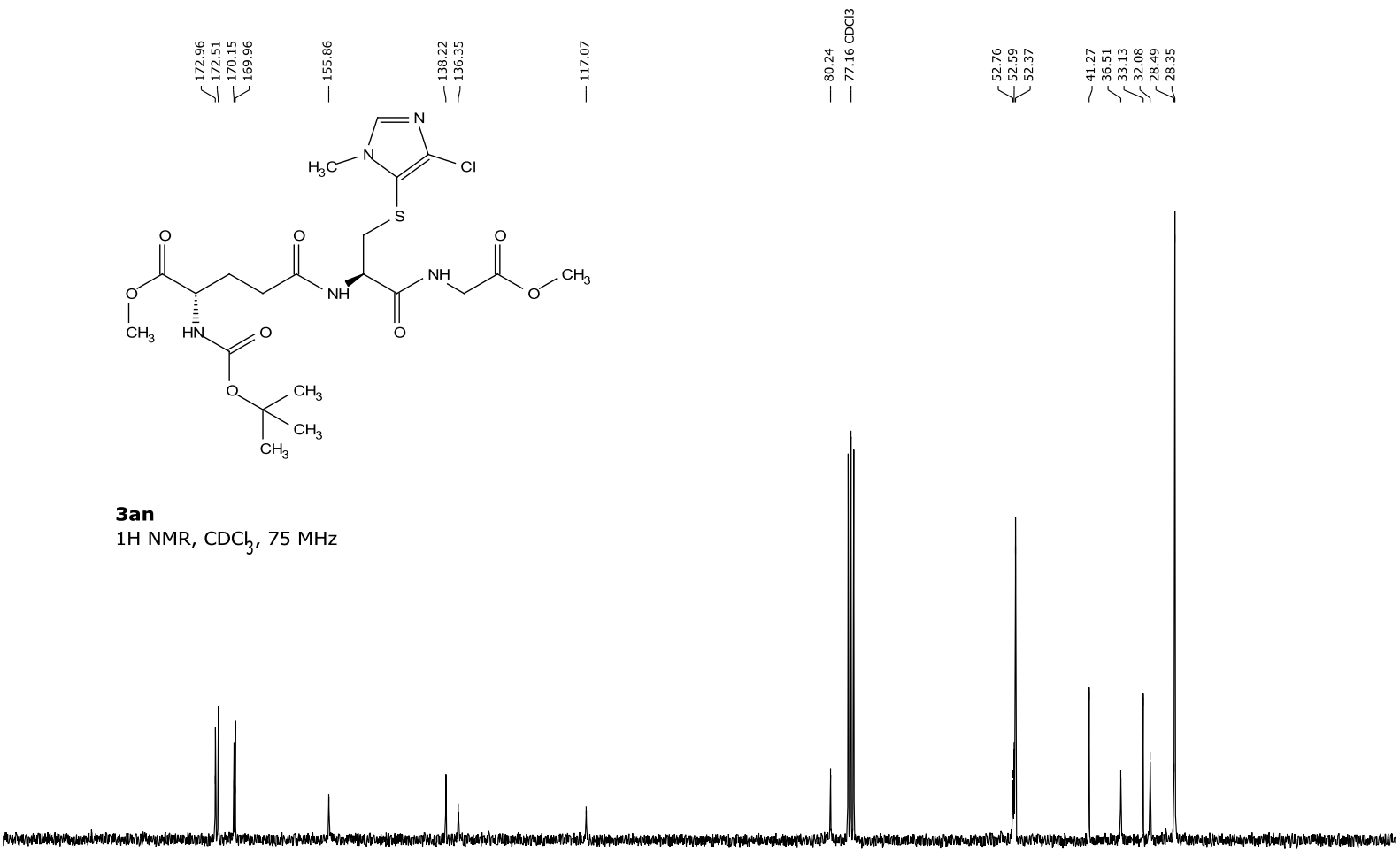

$\begin{array}{llllllllllllllllllllllll}200 & 190 & 180 & 170 & 160 & 150 & 140 & 130 & 120 & 110 & 100 & 90 & 80 & 70 & 60 & 50 & 40 & 30 & 20 & 10 & 0\end{array}$ 
4-Chloro-1-methyl-5-(methylthio)-1H-imidazole (6a)

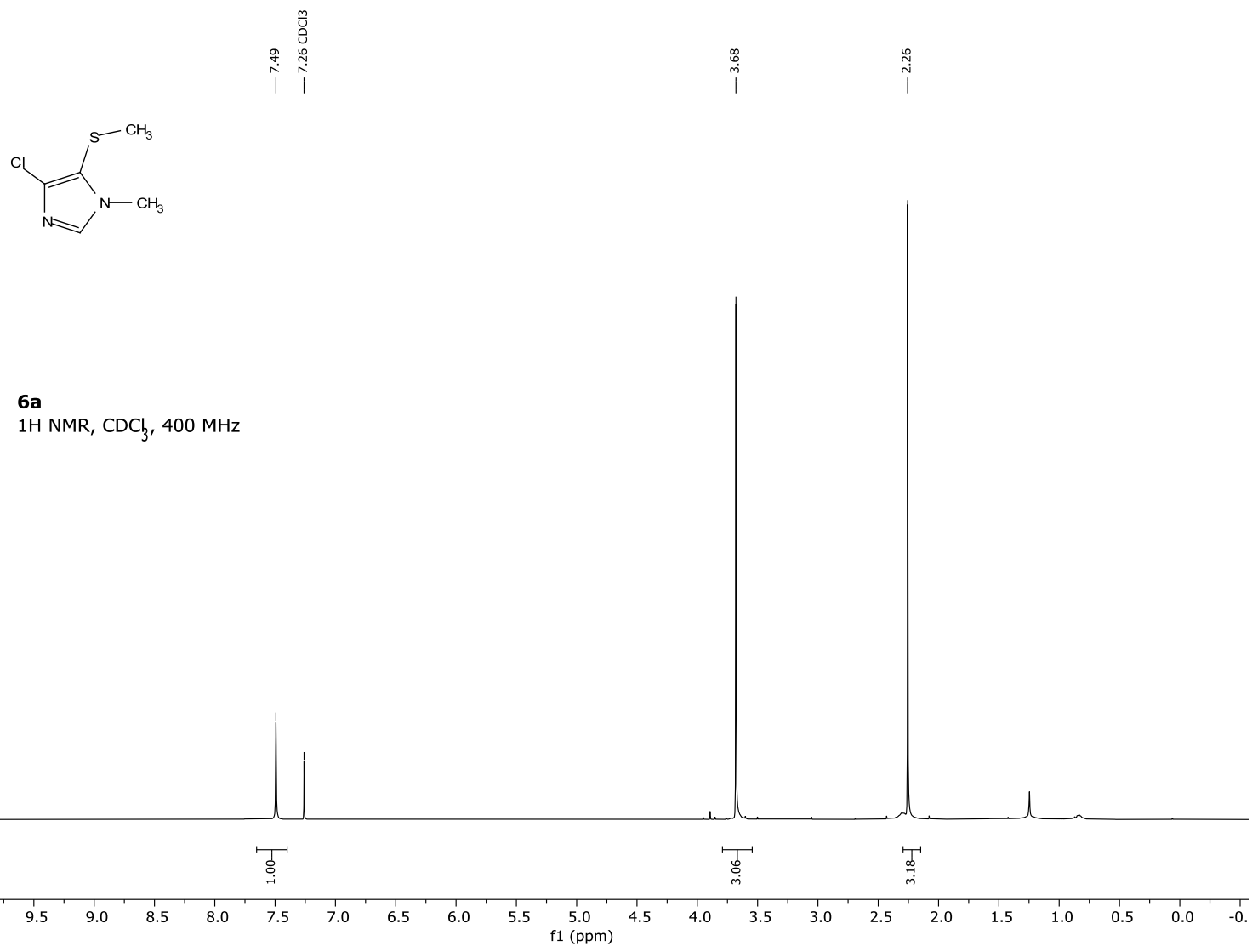

il

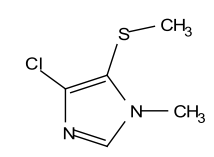

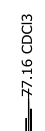

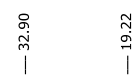

$6 a$

13C NMR, $\mathrm{CDCl}_{3}, 101 \mathrm{MHz}$

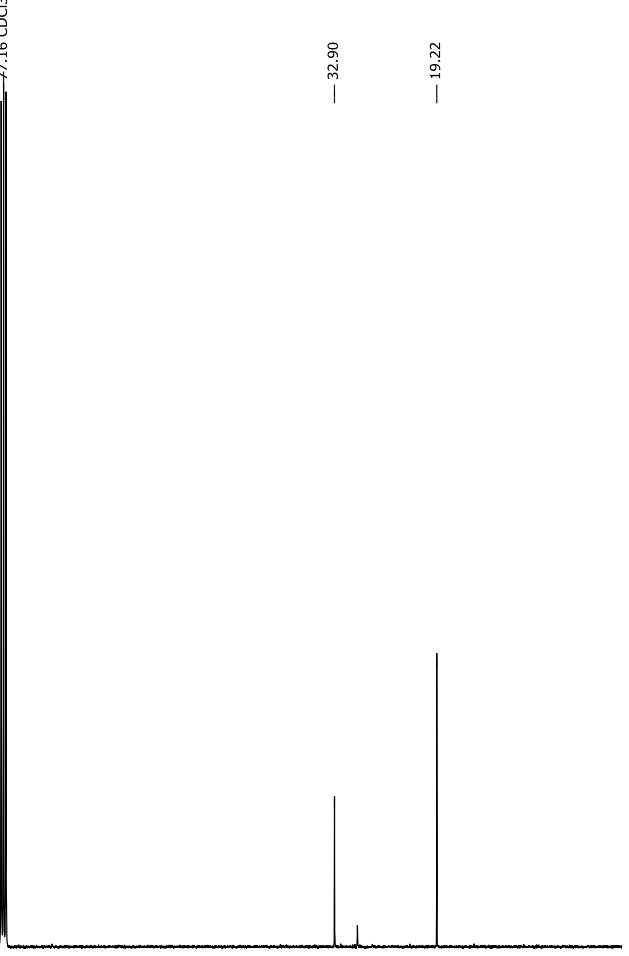

200

$190 \quad 180$

$\begin{array}{llll}170 & 160 & 150\end{array}$

140

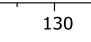

$120 \quad 110$ 
2,4-Dibromo-1-methyl-5-(methylthio)-1H-imidazole (6b)
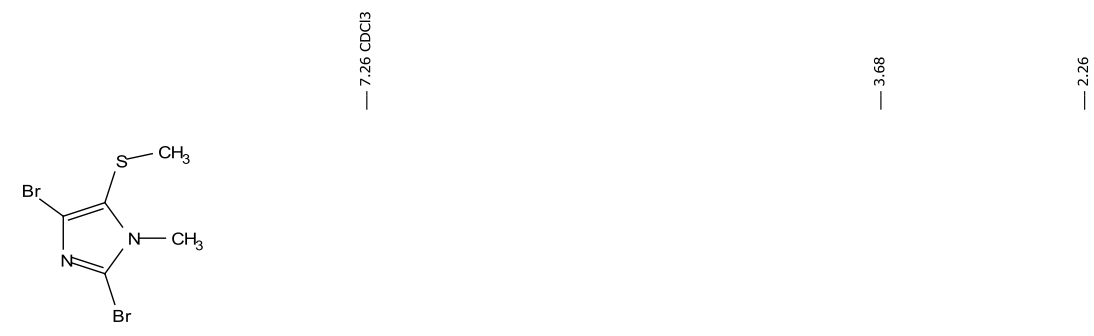

$6 b$

$1 \mathrm{H} \mathrm{NMR}, \mathrm{CDCl}_{3}, 300 \mathrm{MHz}$

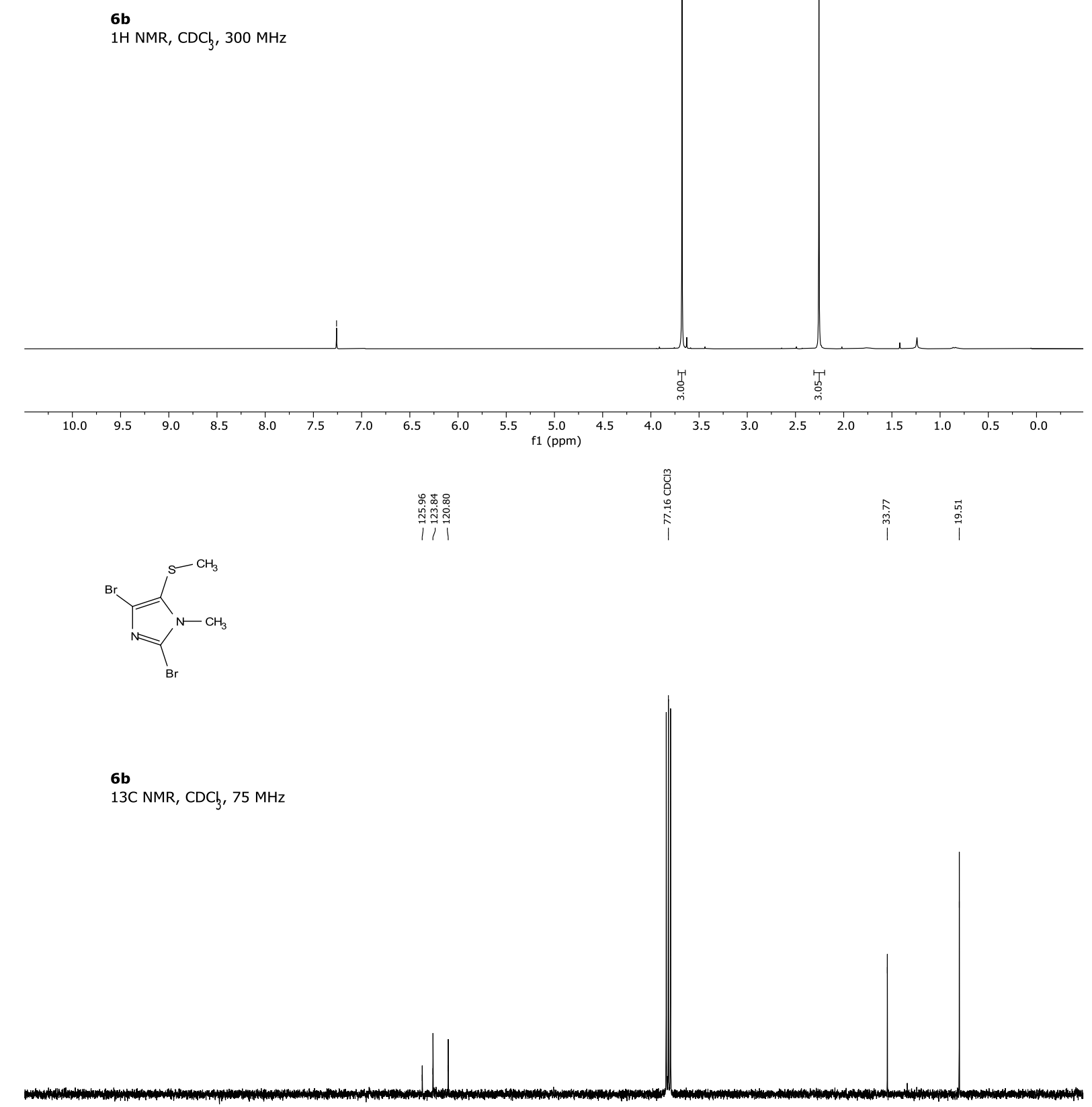

$\begin{array}{lllllllllllllllllllll}1 & 1 \\ 200 & 190 & 180 & 170 & 160 & 150 & 140 & 130 & 120 & 110 & 100 & 90 & 80 & 70 & 60 & 50 & 40 & 30 & 20 & 10 & 0\end{array}$ 
5-(Methylthio)-4-phenylthiazol-2-amine (6c)

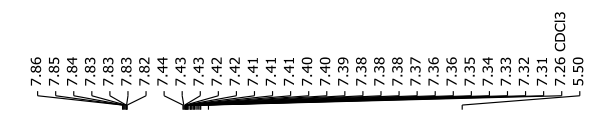<smiles>CSc1sc(N)nc1-c1ccccc1</smiles>

6c

$1 \mathrm{H}$ NMR, $\mathrm{CDCl}_{3}, 300 \mathrm{MHz}$
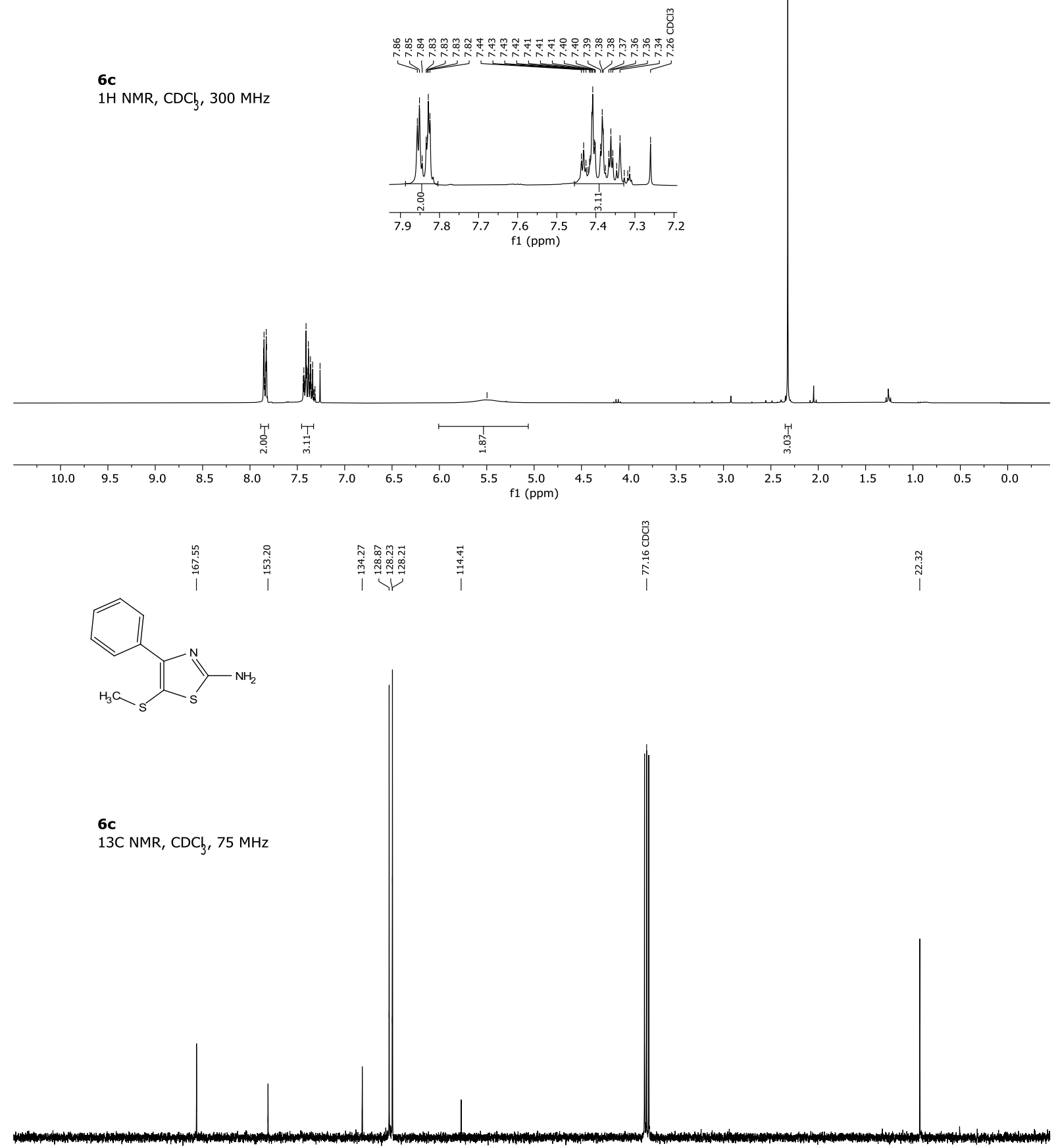

200
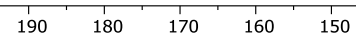

140

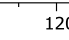


Methyl 3-((4-((4,5-dichloro-1H-imidazol-1-yl)methyl)phenyl)thio)propanoate (4)
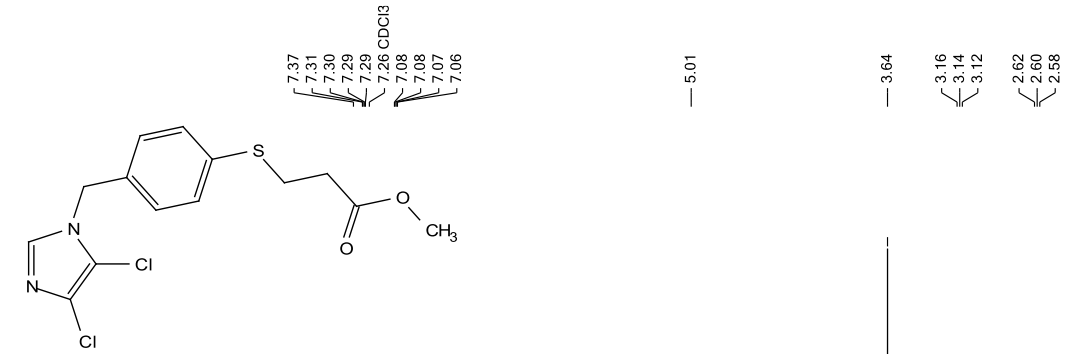

$1 \mathrm{H} \mathrm{NMR}, \mathrm{CDCl}_{3}, 400 \mathrm{MHz}$
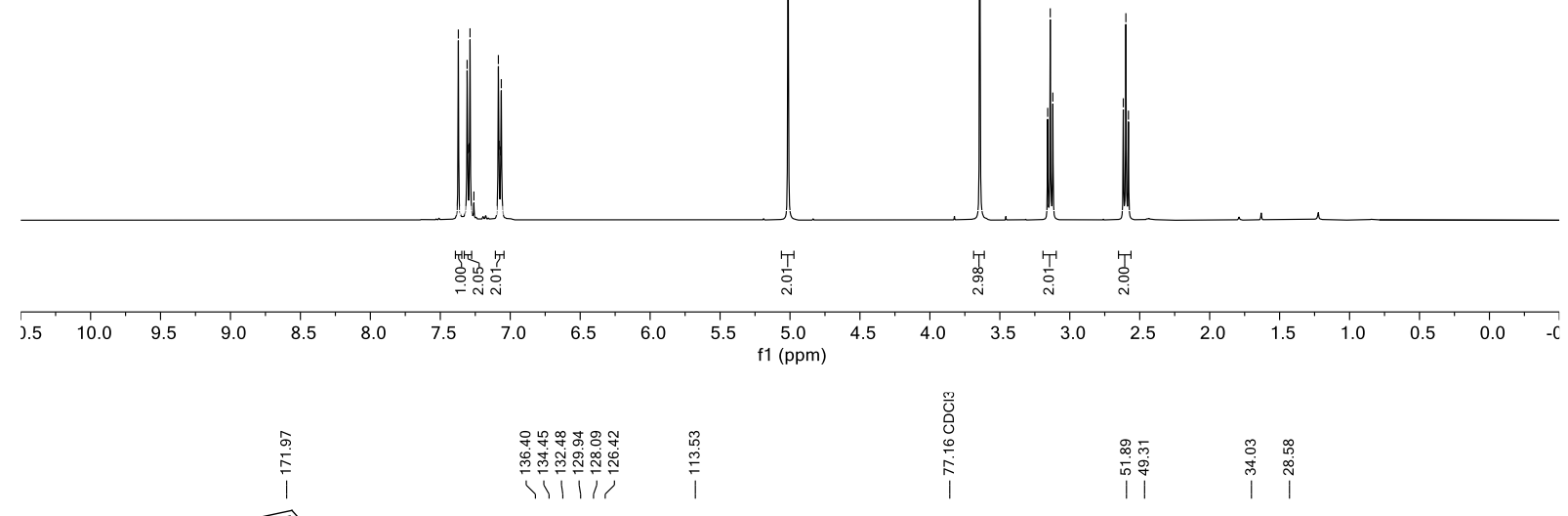

4 13C NMR, $\mathrm{CDCl}_{3}, 101 \mathrm{MHz}$

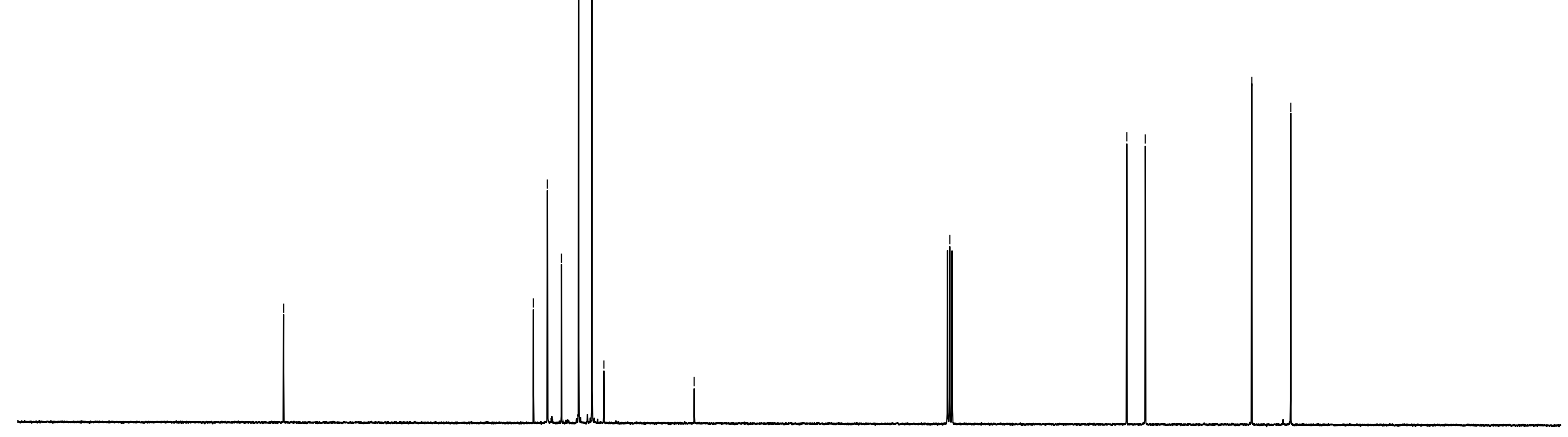

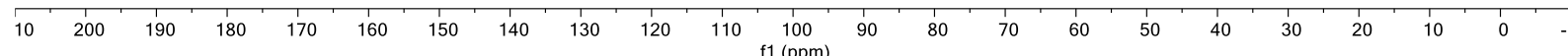


Methyl

3-((5-chloro-1-(4-((3-methoxy-3-oxopropyl)thio)benzyl)-1H-imidazol-4-

yl)thio)propanoate (SI-1)
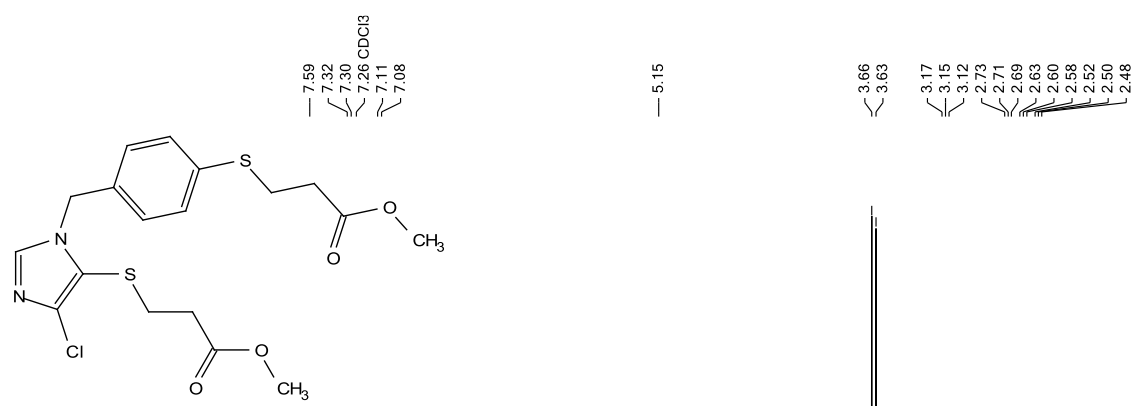

SI-1

$1 \mathrm{H}$ NMR, $\mathrm{CDCl}_{3}, 300 \mathrm{MHz}$

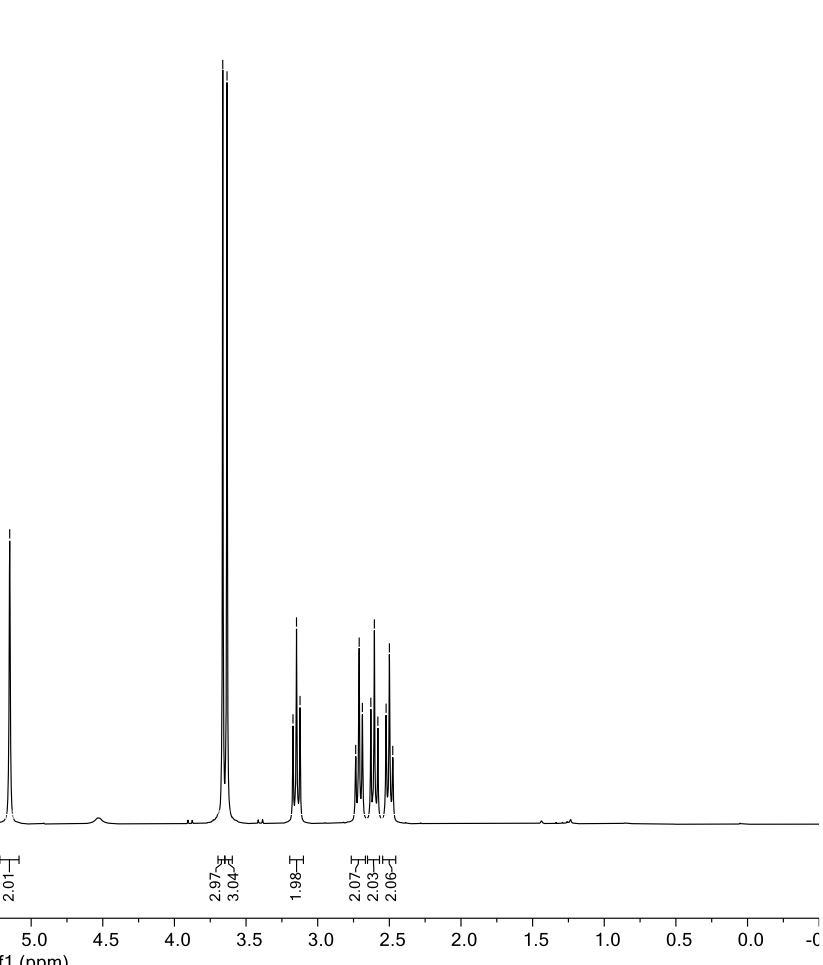


Methyl 3-(octylthio)propanoate (8)

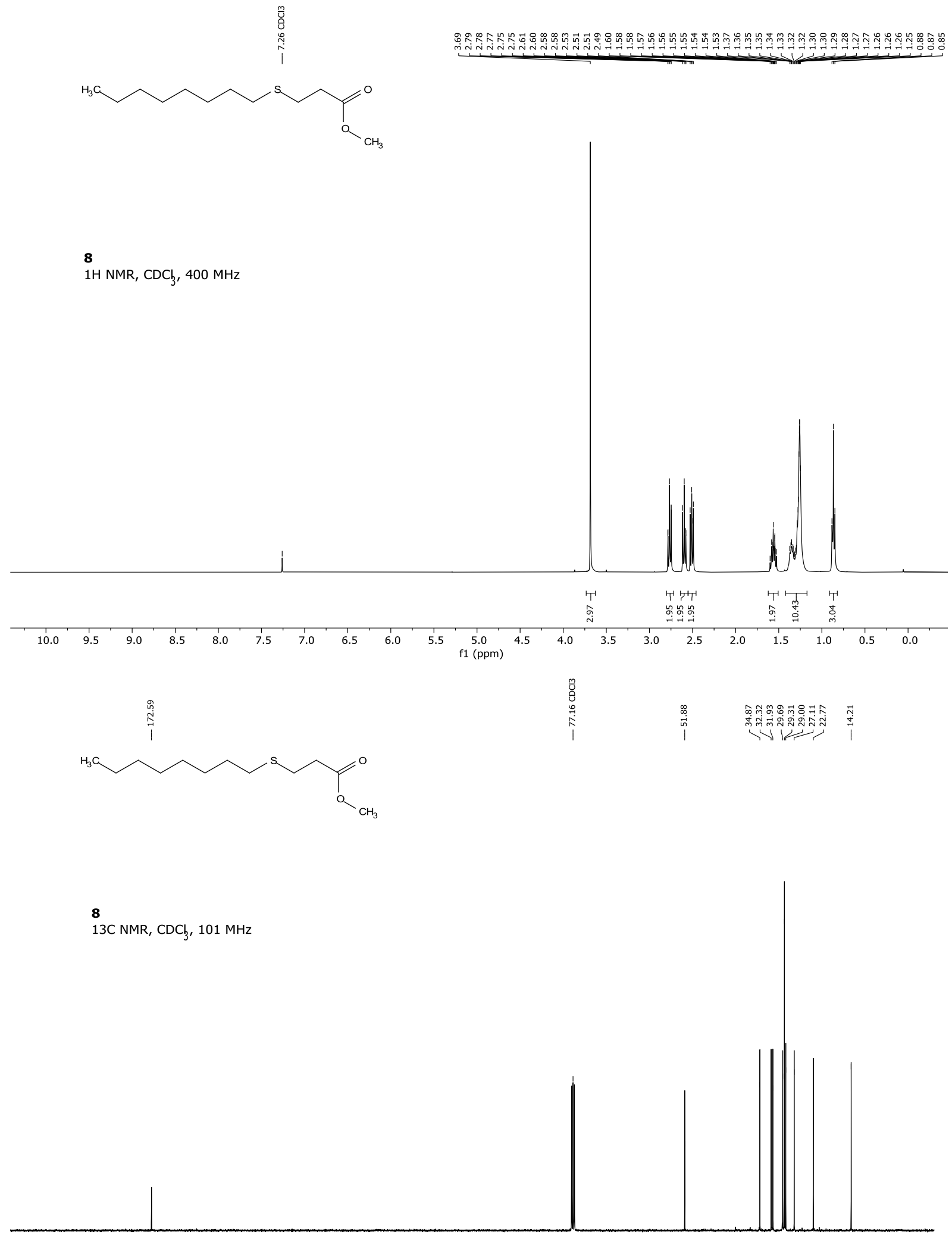

200

$180 \quad 170 \quad 160$

14

$\begin{array}{llll}130 & 120 & 110 & \begin{array}{c}100 \\ \mathrm{f} 1(\mathrm{ppm})\end{array}\end{array}$ 


\section{References}

31. APEX3 (2016), SAINT (2015) and SADABS (2015), Bruker AXS Inc., Madison, Wisconsin, USA.

32. Sheldrick, G. M. SHELXT - Integrated space-group and crystal-structure determination. Acta Cryst. 2015, A71, 3-8.

33. Sheldrick, G.M. Crystal structure refinement with SHELXL. Acta Cryst. 2015, C71 (1), 3-8.

34. XP - Interactive molecular graphics, Version 5.1, Bruker AXS Inc., Madison, Wisconsin, USA, 1998.

35. Hanss, D.; Freys, J. C.; Bernardinelli, G.; Wenger, O. S. Eur. J. Org. Chem. 2009, 48504853.

36. Slinker, J. D.; Gorodetsky, A. A.; Lowry, M. S.; Wang, J.; Parker, S.; Rohl, R.; Bernhard, S.; Malliaras, G. G. J. Am. Chem. Soc. 2004, 126, 2763-2767.

37. Tamayo, A. B.; Alleyne, B. D.; Djurovich, P. I.; Lamansky, S.; Tsyba, I.; Ho, N. N.; Bau, R.; Thompson, M. E. J. Am. Chem. Soc. 2003, 125, 7377-7387.

38. Bouzaid, J.; Schultz, M.; Lao, Z.; Bartley, J.; Bostrom, T.; McMurtrie, J. Cryst. Growth Des. 2012, 12, 3906-3916.

39. Luo, J.; Zhang, J. ACS. Catal. 2016, 6, 873-877.

40. Liégault, B., Petrov, I. Gorelsky, S. I., Fagnou, K. J. Org. Chem. 2010, 75, 1047-1060.

41. M. Buchholz, U. heiser, S. Schilling, A. J. Niestroj, K. Zunkel, H.-U. Demutz, J. Med. Chem. 2006, 49, 664-677.

42. Wright, W. B., Press, J. B., Chan, P. S.; Marsico, J. W.; Haug, M. F.; Lucas, J.; Tauber, J.; Tomcufcik, A. S. J. Med. Chem. 1986, 29, 523-530.

43. Guillen, F.; Brégeon, D.; Plaquevent, J.-C. Tetrahedron Lett. 2006, 47, 1245-1248.

44. Hazra, C. K.; Gandhamsetty, N.; Park, S., Chang, S. Nat. Commun. 2016, 7, 13431.

45. Kikelj, D.; Urleb, U. Sci. Synth. 2002, 11, 627-833.

46. Holzwarth, M. S.; Frey, W.; Plietker, B. Chem. Commun. 2011, 47, 11113-11115.

47. Susumu, K.; Uyeda, H. T.; Medintz, I. L.; Pons, T., Delehanty, J. B.; Mattoussi, H. J. Am. Chem. Soc. 2007, 129, 13987-13996.

48. Janssen-Müller, D.; Singha, S.; Lied, F.; Gottschalk, K.; Glorius, F. Angew. Chem., Int. Ed. 2017, 56, 6276-6279.

49. Cismesia, M. A.; Yoon, T. P. Chem. Sci. 2015, 6, 5426-5434.

50. a) Hatchard, C. G.; Parker, C. A. Proc. Roy. Soc. A (London) 1956, 235, 518-536. b) Kuhn, H. J.; Braslavsky, S. E.; Schmidt, R. Pure Appl. Chem. 2004, 76, 2105-2146. c) Monalti, M.; Credi, A.; Prodi, L.; Gandolfi, M. T.; Chemical Actinometry. Handbook of Photochemistry, 3rd Ed; Taylor \& Francis Group, LLC. Boca Raton, FL, 2006, 601.

51. Wegner, E. E.; Adamson, A. W. J. Am. Chem. Soc. 1966, 88, 394-404.

52. a) Neese, F. WIREs Comput. Mol. Sci. 2012, 2, 73-78. b) Neese, F. WIREs Comput. Mol. Sci. 2018, 8, e1327.

53. Yanai, T.; Tew, D. P.; Handy, N. C. Chem. Phys. Lett. 2004, 393, 51-57.

54. a) Grimme, S.; Ehrlich, S.; Goerigk, L. J. Comput. Chem. 2011, 32, 1456-1465. b) Grimme, S.; Anthony, J.; Ehrlich, S.; Krieg, H. J. Chem. Phys. 2010, 132, 154104. 
55. Weigend, F.; Ahlrichs, R. Phys. Chem. Chem. Phys. 2005, 7, 3297-3305.

56. a) Barone, V.; Cossi, M. J. Phys. Chem. A 1998, 102, 1995-2001. b) Cossi, M.; Rega, N.; Scalmani, G.; Barone, V. J. Comput. Chem. 2003, 24, 669-681.

57. Jmol: an open-source Java viewer for chemical structures in 3D. http://www.jmol.org 58. Gentry, E. C.; Knowles, R. R. Acc. Chem. Res. 2016, 49, 1546-1556.

59. Roth, H. G.; Romero, N. A.; Nicewicz, D. A. Synlett 2016, 27, 714-723.

60. Isse, A. A., Gennaro, A. J. J. Phys Chem. B 2010, 114, 7894-7899. 